\title{
Monolayer functionalization of silicon micro and nanowires: \\ towards solar-to-fuel and sensing devices
}





\section{MONOLAYER FUNCTIONALIZATION OF \\ SILICON MICRO AND NANOWIRES: \\ TOWARDS SOLAR-TO-FUEL AND SENSING DEVICES}

Janneke Veerbeek 
Members of the committee:

$\begin{array}{lll}\text { Chairman: } & \text { prof. dr. ir. J.W.M. Hilgenkamp } & \text { University of Twente } \\ \text { Promotor: } & \text { prof. dr. ir. J. Huskens } & \text { University of Twente } \\ \text { Members: } & \text { prof. dr. J.G.E. Gardeniers } & \text { University of Twente } \\ & \text { prof. dr. S. Hecht } & \text { Humboldt-Universität zu Berlin } \\ & \text { prof. dr. S.J.G. Lemay } & \text { University of Twente } \\ & \text { prof. dr. G. Mul } & \text { University of Twente } \\ & \text { prof. dr. J.N.H. Reek } & \text { University of Amsterdam }\end{array}$

The research described in this thesis was performed within the laboratories of the Molecular NanoFabrication (MnF) group, the MESA+ Institute for Nanotechnology, and the Department of Science and Technology (TNW) of the University of Twente (UT). This research was supported by the Netherlands Organization for Scientific Research (NWO, MESA+ School for Nanotechnology, grant 022.003.001).
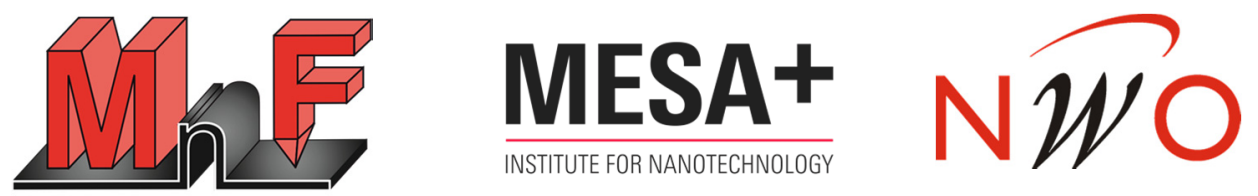

Monolayer functionalization of silicon micro and nanowires: towards solar-to-fuel and sensing devices

Copyright (C) 2017 Janneke Veerbeek

PhD thesis, University of Twente, Enschede, the Netherlands

ISBN: $\quad 978-90-365-4308-8$

DOI: $\quad 10.3990 / 1.9789036543088$

Cover art: Janneke Veerbeek

Printed by: Gildeprint 


\title{
MONOLAYER FUNCTIONALIZATION OF SILICON MICRO AND NANOWIRES: \\ TOWARDS SOLAR-TO-FUEL AND SENSING DEVICES
}

\author{
PROEFSCHRIFT
}

\author{
ter verkrijging van \\ de graad van doctor aan de Universiteit Twente, \\ op gezag van de rector magnificus, \\ prof. dr. T.T.M. Palstra, \\ volgens besluit van het College voor Promoties \\ in het openbaar te verdedigen \\ op vrijdag 12 mei 2017 om 14:45 uur
}

door

Janneke Veerbeek

geboren op 28 februari 1990

te Noordoostpolder, Nederland 
Dit proefschrift is goedgekeurd door:

Promotor: $\quad$ prof. dr. ir. J. Huskens 


\section{Table of contents}

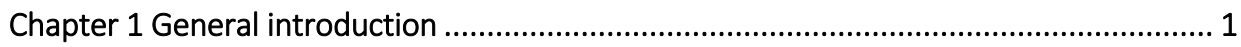

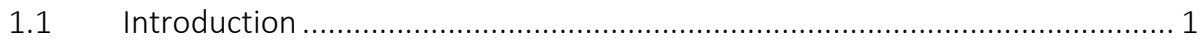

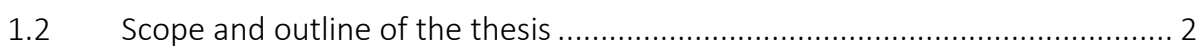

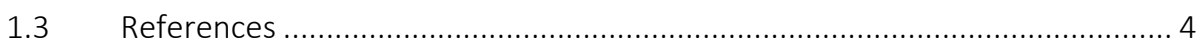

Chapter 2 Applications of monolayer-functionalized $\mathrm{H}$-terminated silicon surfaces: a

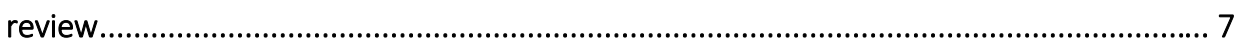

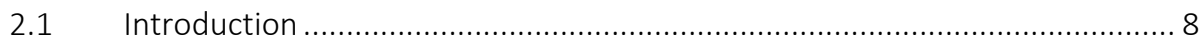

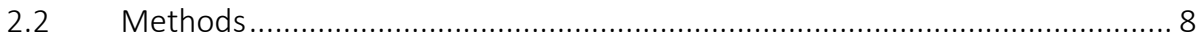

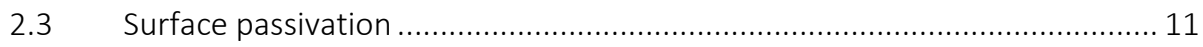

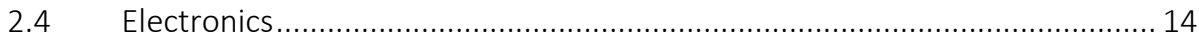

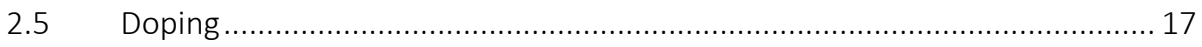

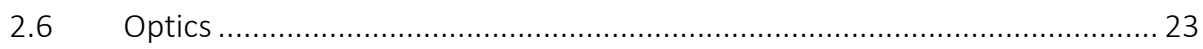

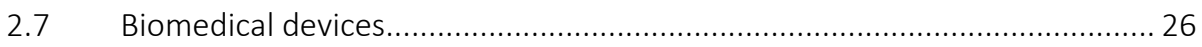

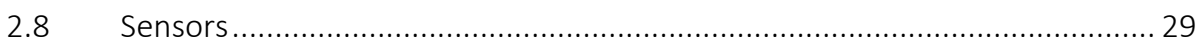

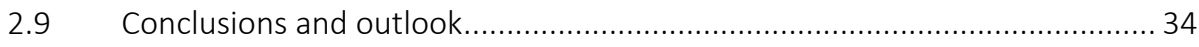

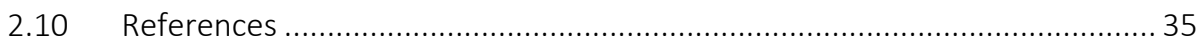

Chapter 3 Molecular monolayers for electrical passivation and functionalization of silicon-

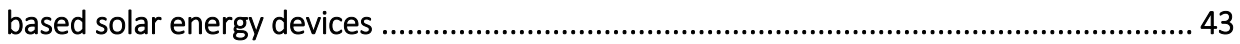

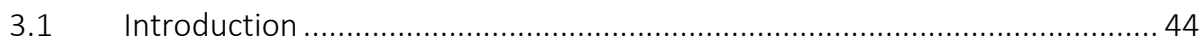

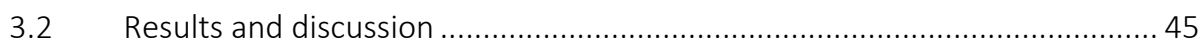

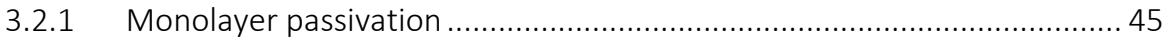

3.2.2 Dual passivation and functionalization ……………………................. 51

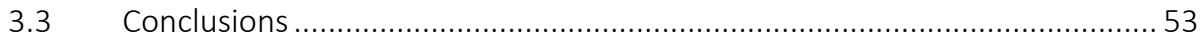

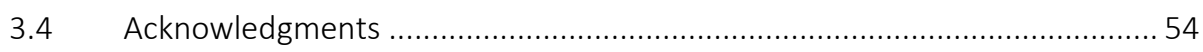

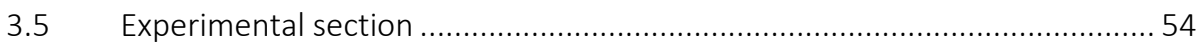

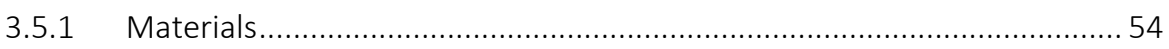

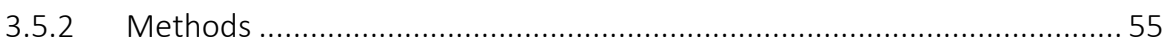

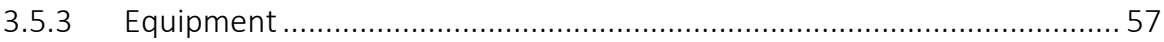

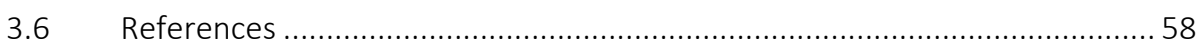

Chapter 4 Highly doped silicon nanowires by monolayer doping .................................... 61

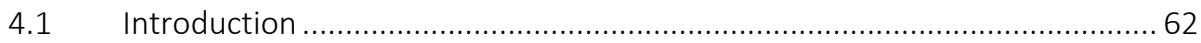

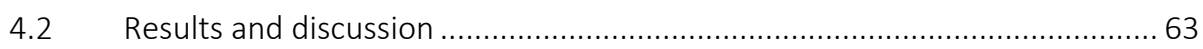

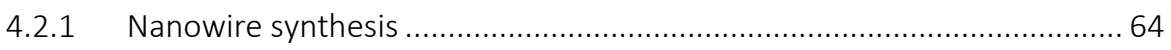




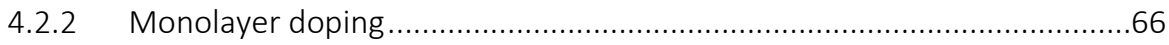

4.2.3 Monolayer contact doping...................................................................68

4.2.4 Monolayer doping with an external capping layer ...................................70

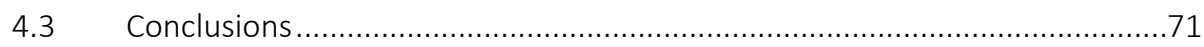

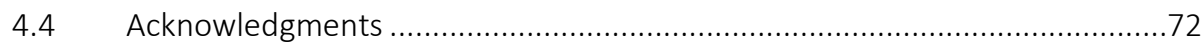

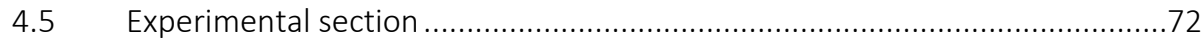

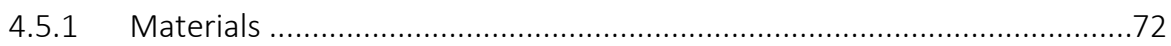

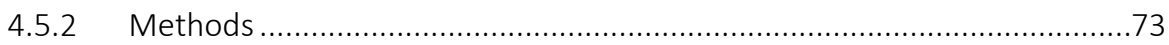

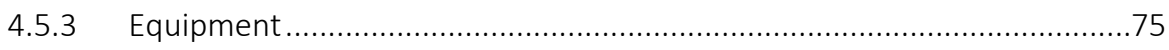

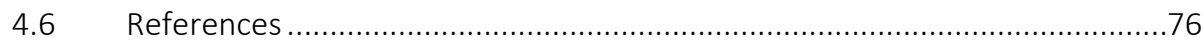

Chapter 5 Maskless spatioselective functionalization of silicon nanowires ......................79

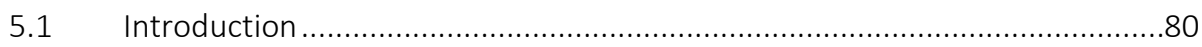

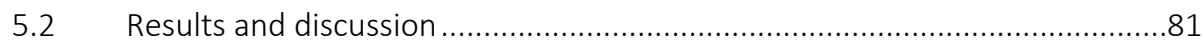

5.2.1 First MACE step and monolayer formation ..........................................83

5.2.2 Second MACE step and monolayer formation ........................................85

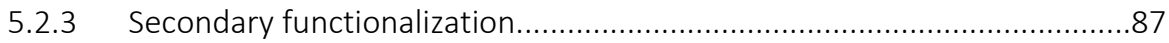

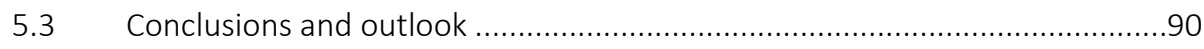

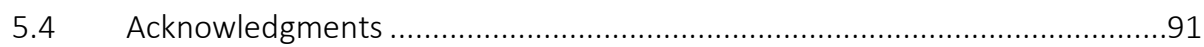

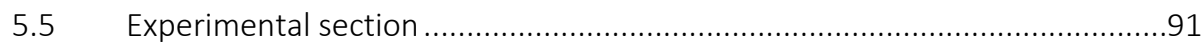

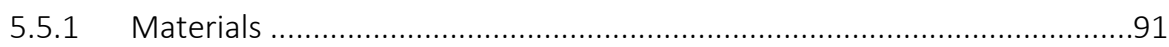

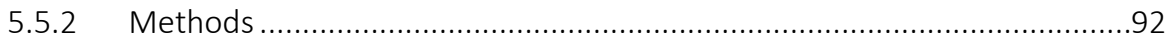

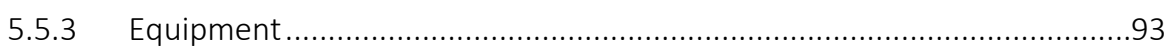

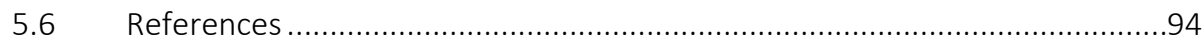

Chapter 6 Selective silicon nanowire functionalization: towards early cancer DNA

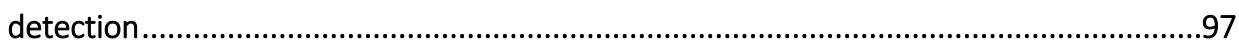

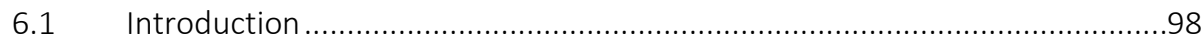

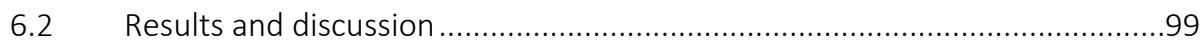

6.2.1 Material-selective monolayer formation .................................................100

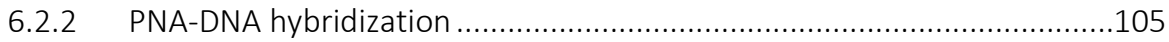

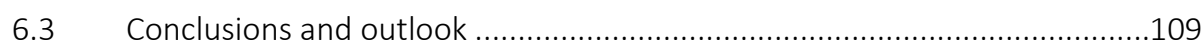

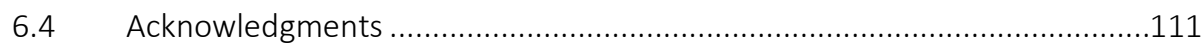

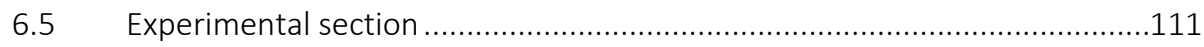

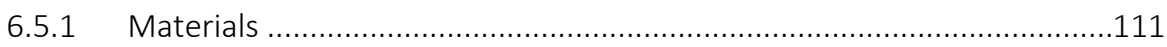

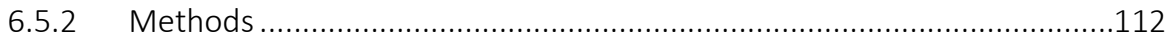

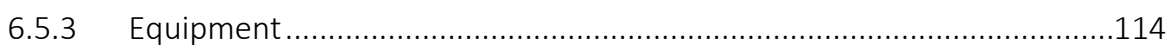

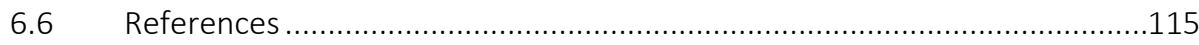


Chapter 7 Electrochemistry of redox-active guest molecules at $\beta$-cyclodextrin-

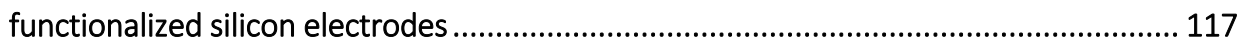

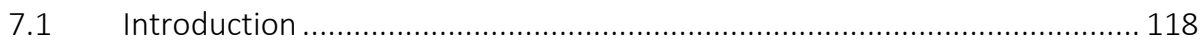

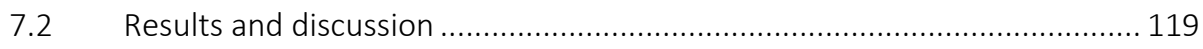

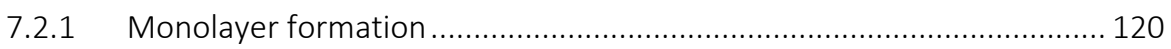

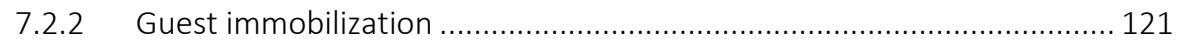

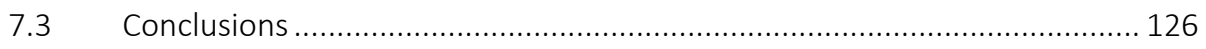

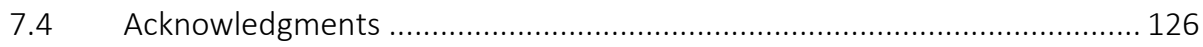

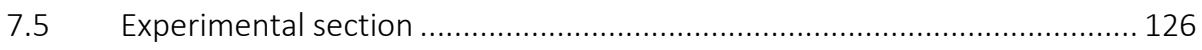

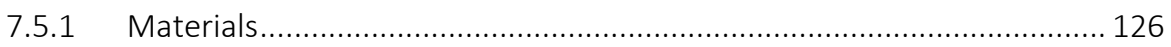

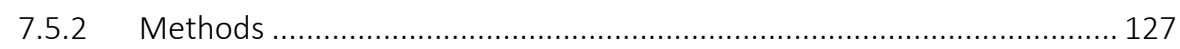

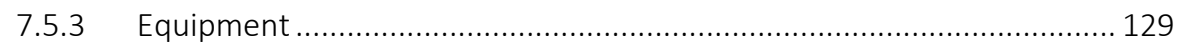

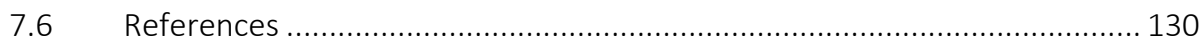

Chapter 8 Covalent and noncovalent immobilization of hydrogen evolution catalysts on

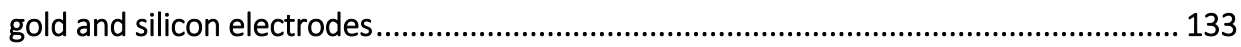

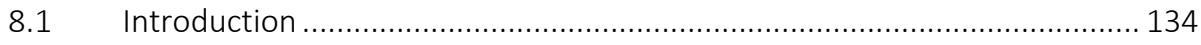

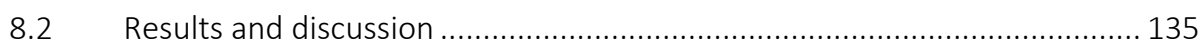

8.2.1 Covalently bound hydrogenase mimic............................................... 137

8.2.2 Supramolecularly bound hydrogenase mimic ..................................... 138

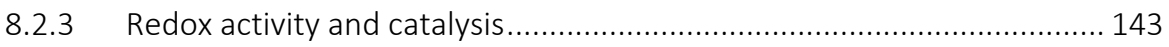

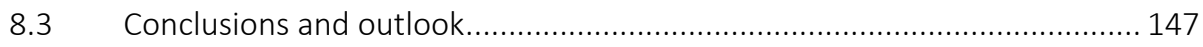

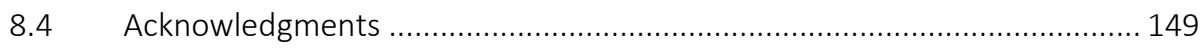

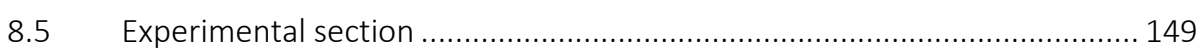

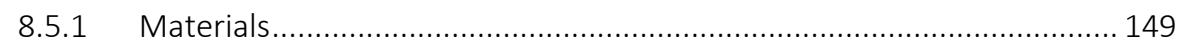

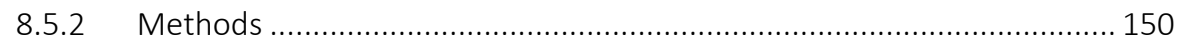

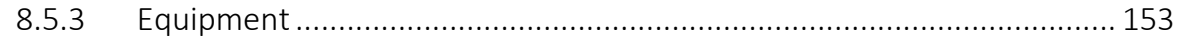

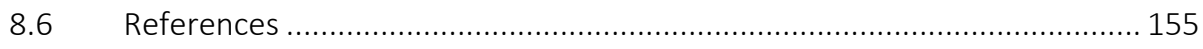

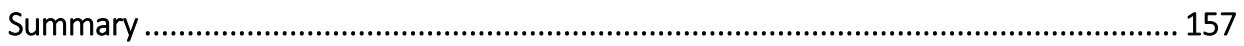

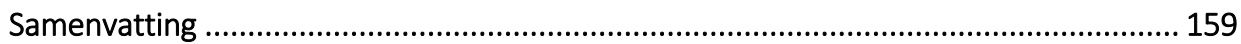

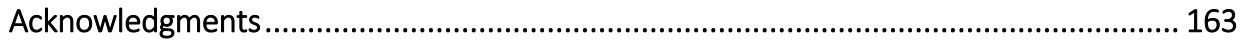

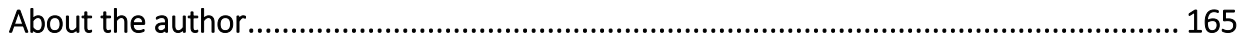

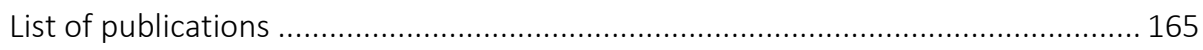




\section{Chapter 1}

\section{General introduction}

\subsection{Introduction}

Silicon (Si) is an earth-abundant material that is commonly used for electronic and energy applications owing to the ease of fabricating silicon micro and nanostructures, the large availability of doping methods, and its attractive semiconductor properties. ${ }^{1}$ Silicon nanostructures and micro/nanowire arrays are of high interest because of their onedimensional architecture and unique optical, electronic, mechanical, and thermal properties. $^{2-4}$

Surface chemistry is a feasible way to tune the functionality of silicon structures towards a specific device, for example by coupling photocatalysts onto the surface for hydrogen production $^{5,6}$ or to make an analyte-specific sensor.,8 Several routes have been investigated for self-assembled monolayer formation on silicon substrates, i.e., the covalent coupling of a single layer of (in)organic molecules. ${ }^{9-11}$ The most commonly used chemistry includes the use of silane derivatives, which bind to $\mathrm{Si}-\mathrm{OH}$ groups at the surface. ${ }^{12}$ These silane-based monolayers are, however, susceptible to hydrolysis. ${ }^{13}$ Moreover, in several applications of silicon-based substrates, the presence of silicon oxide leads to lower quality or nonfunctioning devices. These applications require the removal of the, often electrically insulating, silicon oxide between the silicon surface and the monolayer, thus precluding the more conventional silane-based chemistry.

Oxide-free monolayers can be formed by several routes, of which the two main routes include hydrosilylation ${ }^{14-16}$ and chlorination/alkylation. ${ }^{17,18}$ In this thesis, we focus on the hydrosilylation route because of the possibility to couple versatile molecules in a onestep reaction and the relatively mild reaction conditions needed. By this method, molecules with terminal unsaturated carbon-carbon bonds, i.e., 1-alkenes or 1-alkynes, are coupled onto hydrogen-terminated silicon, and $\mathrm{Si}-\mathrm{C}$ bonds are directly obtained. $\mathrm{H}$-terminated silicon is created by removing the native oxide layer, which is mostly achieved by wet chemistry with an aqueous hydrogen fluoride (HF) solution.

One of the application areas that benefit from oxide-free monolayers include solar-tofuel devices. Because of the world's increasing energy demand, the simultaneous 
depletion of fossil fuels and the climate change induced by increased $\mathrm{CO}_{2}$ emissions, more (versatile) sustainable energy sources are desperately needed. Although silicon solar cells can be used to harvest sunlight for sustainable electricity production, the intermittent presence of sunlight does not allow for a steady power output. Therefore, the production of solar fuels using sunlight has great potential ${ }^{19,20}$ and can lead to hydrogen after water splitting 21,22 or to carbon-based fuels, such as $\mathrm{CO}, \mathrm{CH}_{4}$, or $\mathrm{CH}_{3} \mathrm{OH}$, from $\mathrm{CO}_{2}$ reduction. ${ }^{23}$ To obtain the most efficient, integrated solar-to-fuel device, both an efficient solar cell and catalysts coupled to the surface are required. Here, oxide-free functionalization is advantageous because of an improved electrical contact between the monolayer/catalyst and the substrate, and a higher resistance against oxidation in aqueous environments and air. ${ }^{24}$ Next, structuring of silicon solar cells leads to a higher surface area for capturing more sunlight and a higher loading capacity of catalyst.25,26 Inevitably, micro/nanostructuring also increases the amount of dangling bonds at the surface, which lowers the output of the solar cell due to undesired recombination sites for electron-hole pairs. ${ }^{27}$ A passivation layer, which could also consist of molecular monolayers, ${ }^{28}$ can be used to remove the dangling bonds and boost the solar cell output. ${ }^{29,30}$

Sensing devices also profit by the formation of self-assembled monolayers without silicon oxide. Chemical sensing devices convert the recognition process between a receptor and an analyte into an analytical signal, which can be monitored by, e.g., a variation in fluorescence, ${ }^{31}$ optical $^{32}$ or electrical ${ }^{33}$ properties, or a change in mass. ${ }^{34}$ Signal transduction from/to the substrate is better for $\mathrm{Si}-\mathrm{C}$ monolayers compared to silanebased monolayers, which improves the sensor's sensitivity. ${ }^{35}$ In biosensing applications, an additional driver for oxide-free functionalization includes the enhanced stability in aqueous environments, as Si-O bonds can easily hydrolyze. ${ }^{13}$ Next to a higher sensitivity and stability, the monolayers can also be used to enhance the selectivity of the sensor, i.e., to respond to a specific analyte only. ${ }^{7,8}$

\subsection{Scope and outline of the thesis}

The research described in this thesis aims at the formation of molecular monolayers on $\mathrm{H}$-terminated silicon micro and nanowires for solar-to-fuel devices (Chapters 3, 4, 5, and 8) and sensing devices (Chapters 6 and 7). For solar-to-fuel devices, monolayers are studied for passivation, doping, spatioselective functionalization, and catalyst immobilization. For sensing devices, monolayers are investigated to increase the selectivity and sensitivity of a sensor.

Chapter 2 provides a literature overview of the applications of monolayer-functionalized $\mathrm{H}$-terminated silicon surfaces. The different techniques to create $\mathrm{Si}-\mathrm{C}, \mathrm{Si}-\mathrm{N}, \mathrm{Si}-\mathrm{O}-\mathrm{C}$, and Si-S bonds in an oxide-free way are surveyed, of which the most frequently used techniques include hydrosilylation and chlorination/alkylation. The applications of these 
surfaces are reviewed, as subdivided into the areas of surface passivation, electronics, doping, optics, biomedical devices, and sensors.

In Chapter 3, molecular monolayers are used for electrical passivation and simultaneous functionalization of silicon solar cells. Planar and micropillar-based silicon solar cells with planar and radial p-n junctions are fabricated, respectively, and subsequently functionalized with 1-alkynes by hydrosilylation. The passivation effect is characterized by J-V measurements, whereas coupling of a model catalyst is studied by fluorescence microscopy.

In Chapter 4, the fabrication and doping of silicon nanowires is described. Silicon nanowires are fabricated by metal-assisted chemical etching (MACE), after which three different monolayer doping techniques are used to dope the nanowires. The influence of the porosity of the nanowires on the total doping dose is investigated.

In Chapter 5, a method for the spatioselective functionalization of silicon nanowires is tested without the use of a masking material. The designed process is based on alternating steps of MACE to create (parts of) silicon nanowires and hydrosilylation to form $\mathrm{Si}-\mathrm{C}$ monolayers on the exposed silicon parts. Secondary functionalization by click chemistry with azide-functionalized model compounds is tested. The different process parameters that influence the success rate of the selective functionalization process are discussed.

In Chapter 6, selective functionalization of silicon nanowires is tested on a sensor for cancer DNA detection. Absence of a monolayer on the inactive silicon oxide surroundings would prevent loss of analyte and thus increase the sensitivity of the sensor. Hydrosilylation is used to form a 1,8-nonadiyne monolayer, after which the selectivity is imaged by click chemistry with dummy molecules. Moreover, the headgroup of the 1,8-nonadiyne monolayer is functionalized with different PNA probes to test PNA-DNA hybridization.

In Chapter 7, the electronic coupling between a $\beta$-cyclodextrin monolayer and a silicon substrate is studied using redox-active guest molecules. Hydrosilylation is applied to form monolayers of an alkyne-functionalized $\beta$-cyclodextrin molecule, after which host-guest complexes are formed with a ferrocene-containing trivalent guest. The characteristic ferrocene redox signal is monitored by electrochemistry on differently doped silicon substrates.

In Chapter 8, covalent and noncovalent immobilization of hydrogen evolution catalysts is reported. Hydrosilylation is used to covalently couple an alkyne-functionalized catalyst 
onto $\mathrm{H}$-terminated silicon substrates. Next, $\beta$-cyclodextrin monolayers on gold and silicon surfaces are employed to immobilize a guest-functionalized catalyst in a supramolecular way. Electrochemistry is used to characterize the redox properties and catalytic activity of the catalyst after immobilization.

\subsection{References}

1. R. Elbersen, W. Vijselaar, R.M. Tiggelaar, H. Gardeniers and J. Huskens, Adv. Mater., 2015, 27, 6781-6796.

2. N.P. Dasgupta, J.W. Sun, C. Liu, S. Brittman, S.C. Andrews, J. Lim, H.W. Gao, R.X. Yan and P.D. Yang, Adv. Mater., 2014, 26, 2137-2184.

3. Y.L. Wang, T.Y. Wang, P.M. Da, M. Xu, H. Wu and G.F. Zheng, Adv. Mater., 2013, 25, 51775195.

4. K.Q. Peng, X. Wang, L. Li, Y. Hu and S.T. Lee, Nano Today, 2013, 8, 75-97.

5. I. Oh, J. Kye and S. Hwang, Nano Lett., 2012, 12, 298-302.

6. S.Y. Reece, J.A. Hamel, K. Sung, T.D. Jarvi, A.J. Esswein, J.J.H. Pijpers and D.G. Nocera, Science, 2011, 334, 645-648.

7. L. Basabe-Desmonts, J. Beld, R.S. Zimmerman, J. Hernando, P. Mela, M.F. Garcia-Parajo, N.F. van Hulst, A. van den Berg, D.N. Reinhoudt and M. Crego-Calama, J. Am. Chem. Soc., 2004, 126, 7293-7299.

8. M.D. Yilmaz, S.H. Hsu, D.N. Reinhoudt, A.H. Velders and J. Huskens, Angew. Chem., Int. Ed., 2010, 49, 5938-5941.

9. J.J. Gooding and S. Ciampi, Chem. Soc. Rev., 2011, 40, 2704-2718.

10. A. Ulman, Chem. Rev., 1996, 96, 1533-1554.

11. G. Collins and J.D. Holmes, J. Mater. Chem., 2011, 21, 11052-11069.

12. S. Onclin, B.J. Ravoo and D.N. Reinhoudt, Angew. Chem., Int. Ed., 2005, 44, 6282-6304.

13. M.J. Sweetman, F.J. Harding, S.D. Graney and N.H. Voelcker, Appl. Surf. Sci., 2011, 257, 67686774.

14. J.M. Buriak, Chem. Commun., 1999, 1051-1060.

15. N. Shirahata, A. Hozumi and T. Yonezawa, Chem. Rec., 2005, 5, 145-159.

16. S. Ciampi, J.B. Harper and J.J. Gooding, Chem. Soc. Rev., 2010, 39, 2158-2183.

17. M.Y. Bashouti, K. Sardashti, S.W. Schmitt, M. Pietsch, J. Ristein, H. Haick and S.H. Christiansen, Prog. Surf. Sci., 2013, 88, 39-60.

18. K.T. Wong and N.S. Lewis, Acc. Chem. Res., 2014, 47, 3037-3044.

19. M.G. Walter, E.L. Warren, J.R. McKone, S.W. Boettcher, Q.X. Mi, E.A. Santori and N.S. Lewis, Chem. Rev., 2010, 110, 6446-6473.

20. K. Sun, S. Shen, Y. Liang, P.E. Burrows, S.S. Mao and D. Wang, Chem. Rev., 2014, 114, 86628719.

21. P.P. Edwards, V.L. Kuznetsov, W.I.F. David and N.P. Brandon, Energy Policy, 2008, 36, 43564362.

22. J. Nowotny, C.C. Sorrell, L.R. Sheppard and T. Bak, Int. J. Hydrogen Energy, 2005, 30, 521-544.

23. W.G. Tu, Y. Zhou and Z.G. Zou, Adv. Mater., 2014, 26, 4607-4626.

24. M.Y. Bashouti, J. Ristein, H. Haick and S. Christiansen, Hybrid Mater., 2014, 1, 2-14. 
25. R. Elbersen, W. Vijselaar, R.M. Tiggelaar, H. Gardeniers and J. Huskens, Adv. Energy Mater., 2016, 6, 1501728.

26. M.D. Kelzenberg, S.W. Boettcher, J.A. Petykiewicz, D.B. Turner-Evans, M.C. Putnam, E.L. Warren, J.M. Spurgeon, R.M. Briggs, N.S. Lewis and H.A. Atwater, Nat. Mater., 2010, 9, 239244.

27. M.V. Fernandez-Serra, C. Adessi and X. Blase, Nano Lett., 2006, 6, 2674-2678.

28. F. Zhang, D. Liu, Y. Zhang, H. Wei, T. Song and B. Sun, ACS Appl. Mater. Interfaces, 2013, 5, 4678-4684.

29. A.G. Aberle, Prog. Photovoltaics, 2000, 8, 473-487.

30. A.D. Mallorquí, E. Alarcón-Lladó, I.C. Mundet, A. Kiani, B. Demaurex, S. De Wolf, A. Menzel, M. Zacharias and A. Fontcuberta i Morral, Nano Res., 2015, 8, 673-681.

31. E. Biavardi, M. Favazza, A. Motta, I.L. Fragala, C. Massera, L. Prodi, M. Montalti, M. Melegari, G.G. Condorelli and E. Dalcanale, J. Am. Chem. Soc., 2009, 131, 7447-7455.

32. L. De Stefano, L. Rotiroti, I. Rea, L. Moretti, G. Di Francia, E. Massera, A. Lamberti, P. Arcari, C. Sanges and I. Rendina, J. Opt. A: Pure Appl. Opt., 2006, 8, S540-S544.

33. H. Haick, P.T. Hurley, A.I. Hochbaum, P. Yang and N.S. Lewis, J. Am. Chem. Soc., 2006, 128, 8990-8991.

34. V.I. Boiadjiev, G.M. Brown, L.A. Pinnaduwage, G. Goretzki, P.V. Bonnesen and T. Thundat, Langmuir, 2005, 21, 1139-1142.

35. Y.L. Bunimovich, Y.S. Shin, W.S. Yeo, M. Amori, G. Kwong and J.R. Heath, J. Am. Chem. Soc., 2006, 128, 16323-16331. 


\section{Chapter 2}

\section{Applications of monolayer-functionalized H-terminated silicon surfaces: a review}

Silicon is an attractive semiconductor material for wide-ranging applications, from electronics and sensing to solar cells. Functionalization of $\mathrm{H}$-terminated silicon surfaces with molecular monolayers can be used to tune the properties of the material towards a desired application. Several applications require the removal of the, often insulating, silicon oxide between the silicon surface and a monolayer, thus precluding the more conventional silane-based chemistry. This chapter surveys the applications of monolayerfunctionalized silicon surfaces starting from $\mathrm{H}$-terminated silicon. The oxide-free routes available for Si-C, Si-N, Si-O-C, and Si-S bond formation are described, of which the most commonly used techniques include hydrosilylation and a chlorination/alkylation route onto $\mathrm{H}$-terminated silicon. Applications are subdivided into the areas of surface passivation, electronics, doping, optics, biomedical devices, and sensors. Overall, these methods provide great prospects for the development of stabilized silicon micro/nanosystems with engineered functionalities.

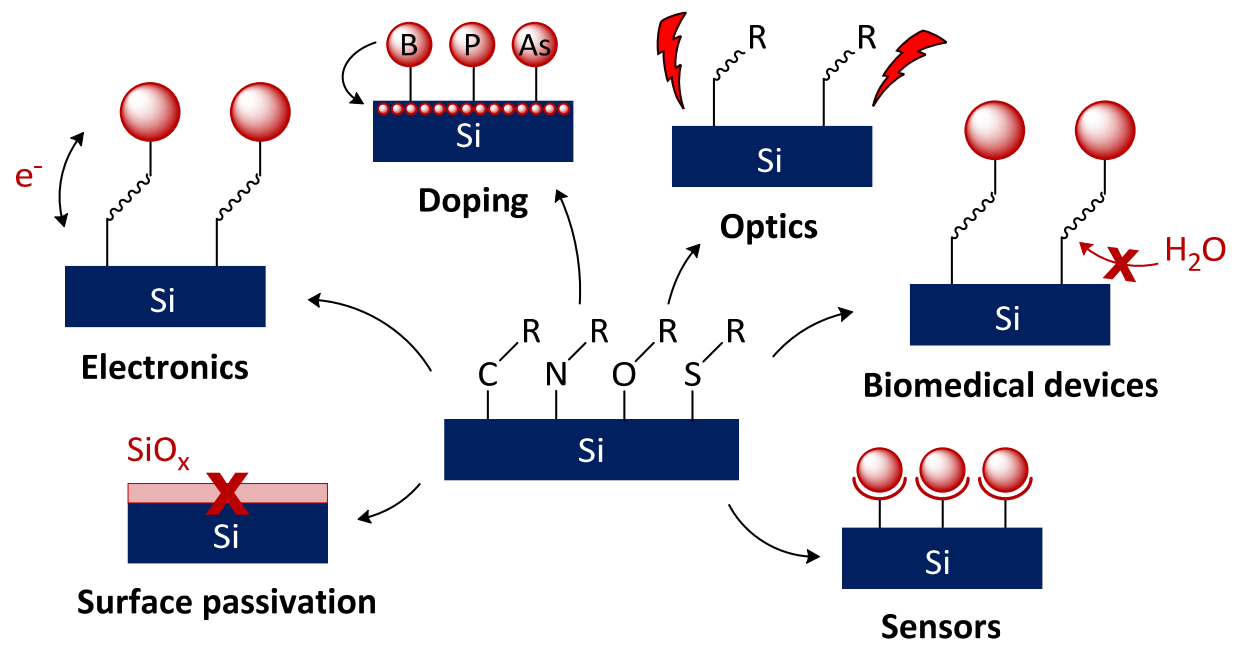

Part of this chapter has been published as: J. Veerbeek and J. Huskens, Small Methods, 2017, 1, 1700072. 


\subsection{Introduction}

Silicon is a commonly used material due to its earth abundance, the availability of fabrication methods and its semiconductor properties. Self-assembled monolayer formation of (in)organic molecules is a feasible way to tune the functionality of the desired substrate towards a specific device. ${ }^{1,2}$ In several fabrication processes of siliconbased devices, the presence of silicon oxide leads to lower quality or nonfunctioning devices. For example, in electronic applications, silicon oxide acts as an insulating layer and should thus be avoided when electrical contact is required. In this chapter, we review the applications of silicon surfaces, i.e., planar substrates, nanowires, and nanoparticles, which require the use of oxide-free functionalization.

Since the formation of Si-C alkyl monolayers originally reported by Linford and Chidsey, ${ }^{3}$ many more routes have been developed. An overview of the routes towards covalent oxide-free formation of molecular monolayers on silicon is given in Section 2.2. This is followed by an overview of the reported applications (Sections 2.3-2.8) in surface passivation, electronics, doping, optics, biomedical devices, and sensors. For each application area, the drivers for oxide-free functionalization are highlighted, of which the main reasons include stability in aqueous environment and air, ${ }^{4}$ and the avoidance of an insulating layer.

\subsection{Methods}

The routes towards covalent, oxide-free functionalization of silicon are summarized only briefly here, since numerous reviews exist on these methods. ${ }^{5-12}$ All routes start from $\mathrm{H}$-terminated $\mathrm{Si}$ and continue with direct or indirect coupling of the desired monolayer (Scheme 2.1). H-terminated $\mathrm{Si}$ is formed by removing the native oxide layer, which is mostly achieved by wet chemistry, although exposure to molecular hydrogen under ultrahigh vacuum is possible as well. ${ }^{6,13}$ In the case of $\mathrm{Si}(111)$ surfaces, immersion in a $40 \%$ aqueous ammonium fluoride $\left(\mathrm{NH}_{4} \mathrm{~F}\right)$ solution results in $\mathrm{Si}$ monohydride sites. For $\mathrm{Si}(100)$, an aqueous $1 \%$ hydrogen fluoride (HF) solution is usually used for $\mathrm{Si}-\mathrm{H}$ formation, which predominantly results in Si dihydride sites, but also monohydride and trihydride sites are formed because of the different crystal lattice compared to Si(111)., 6 


\section{Native oxide removal}

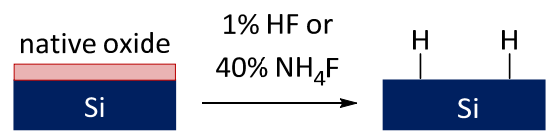

\section{Hydrosilylation}

b
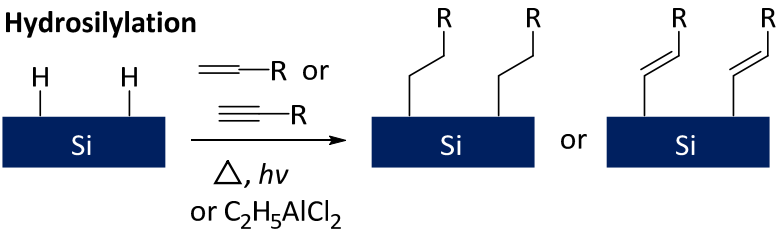

c
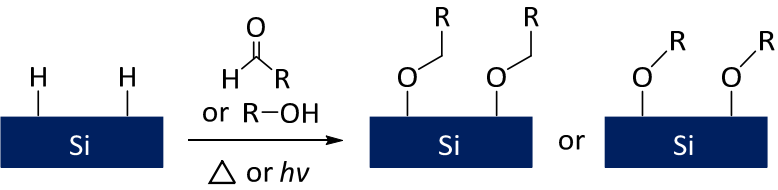

Electrografting

d
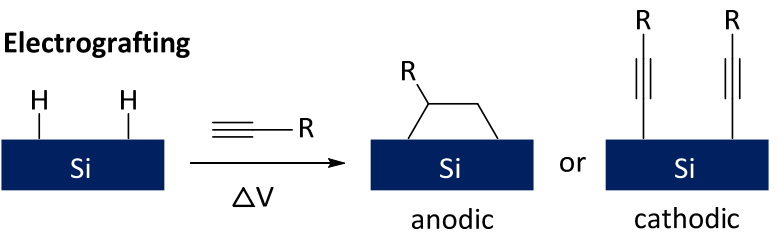

\section{Diazonium salts}

e
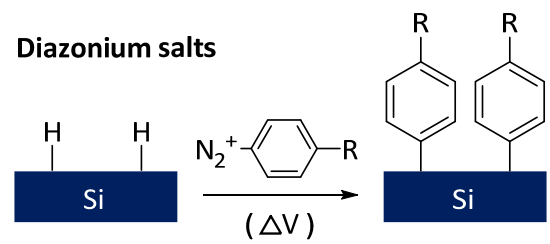

\section{Organochalcogenides}
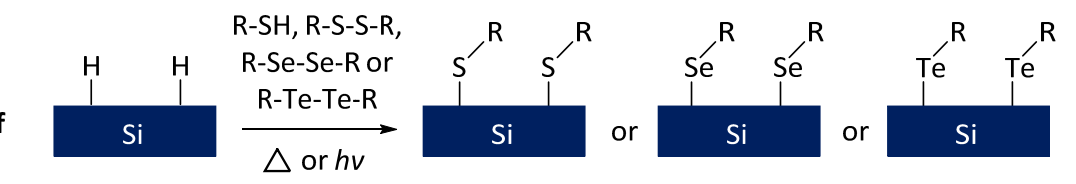

\section{Chlorination/alkylation}

g

$$
\begin{aligned}
& \mathrm{RMgX}(\mathrm{X}=\mathrm{Cl}, \\
& \mathrm{Br}, \mathrm{H}) \text { or } \mathrm{RLi}
\end{aligned}
$$

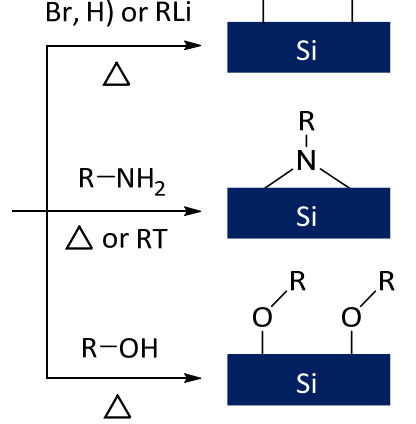

Scheme 2.1. Schematic overview of methods for oxide-free monolayer formation on $\mathrm{H}$-terminated silicon. 
After Si-H formation (Scheme 2.1a), different routes have been reported for monolayer formation (Scheme 2.1b-i). All of these reactions should be performed under water-free and oxygen-free conditions in order to avoid the regrowth of silicon oxide before Si-R bonds are formed. One of the main functionalization routes onto $\mathrm{Si}-\mathrm{H}$ is hydrosilylation, in which unsaturated carbon-carbon bonds, i.e., 1-alkenes or 1-alkynes, are grafted onto $\mathrm{H}$-terminated $\mathrm{Si}$, and $\mathrm{Si}-\mathrm{C}$ bonds are thus directly obtained (Scheme 2.1b). ${ }^{5,6,14}$ For 1-alkenes $\mathrm{Si}-\mathrm{C}-\mathrm{C}-\mathrm{R}$ bonds are obtained, whereas 1 -alkynes result in $\mathrm{Si}-\mathrm{C}=\mathrm{C}-\mathrm{R}$ on $\mathrm{Si}(111)$ and $\mathrm{Si}-\mathrm{C}-\mathrm{C}-\mathrm{R}$ on $\mathrm{Si}(100)$ due to twofold coupling to the Si dihydride sites. ${ }^{5,6}$ The hydrosilylation reaction can be performed under heat (thermal hydrosilylation) ${ }^{15,16}$ or light (photochemical hydrosilylation) $)^{17,18}$ or even in the dark, ${ }^{19,20}$ in diluted ${ }^{21}$ or pure 1-alkene/1-alkyne solutions, ${ }^{15,16}$ optionally in the presence of a Lewis acid catalyst, such as $\mathrm{C}_{2} \mathrm{H}_{5} \mathrm{AlCl}_{2}$. This hydrosilylation route is advantageous due to the direct coupling of versatile molecules and can be performed under mild conditions. Another variant applies an electrical potential to couple 1-alkenes or 1-alkynes to the surface, which is called anodic electrografting when a positive bias is applied and cathodic electrografting when using a negative bias (Scheme 2.1d).22-24 The hydrosilylation reaction can also be applied to bind aldehyde or alcohol molecules onto Si-H directly, which results in Si-O-C linkages (Scheme 2.1c). ${ }^{5,25}$

The other main technique towards oxide-free functionalization includes a chlorination/alkylation route (Scheme $2.1 \mathrm{~g}-\mathrm{i}$ ), in which $\mathrm{H}$-terminated $\mathrm{Si}$ is first converted into $\mathrm{Si}-\mathrm{Cl}$ using $\mathrm{PCl}_{5}$ or chlorine gas. This monolayer subsequently reacts with an alkyl Grignard reagent ( $\mathrm{RMgX}$, with $\mathrm{X}=\mathrm{Cl}, \mathrm{Br}$, or $\mathrm{H}$ ) or an alkyl lithium reagent ( $\mathrm{RLi}$ ) to result in $\mathrm{Si}-\mathrm{C}$ bonds and $\mathrm{MgClX}$ or LiX as a byproduct (Scheme 2.1g). ${ }^{26,27}$ This reaction can also proceed through $\mathrm{Si}-\mathrm{Br}$ or $\mathrm{Si}-\mathrm{I}$ or even directly, ${ }^{6,28}$ but a $\mathrm{Si}-\mathrm{Cl}$ monolayer is the most commonly used intermediate. ${ }^{5}$ The advantage of this route is its ability to use short molecules, for example to make monolayers of single methyl groups. In this case, every $\mathrm{Si}-\mathrm{H}$ site is reacted and thus the surface is fully passivated. ${ }^{6}$ The route can also be used to create $\mathrm{Si}-\mathrm{N}$ bonds by the reaction of ammonia ${ }^{13,29}$ or other primary amines $\left(\mathrm{R}^{-} \mathrm{NH}_{2}\right)$ onto $\mathrm{Si}-\mathrm{Cl}$, where the amino group reacts with two Si-Cl groups and thus forms, at most, a halfpacked monolayer (Scheme 2.1h). 5,30,31 Reacting a primary alcohol (ROH) onto Si-Cl results in a Si-O-C bonded monolayer (Scheme 2.1i). ${ }^{5,32}$

Another option for $\mathrm{Si}-\mathrm{C}$ bond formation includes the reduction of diazonium salts $\left(\mathrm{N}_{2}{ }^{+}\right.$-phenyl-R, with $\mathrm{R}=\mathrm{Br}, \mathrm{NO}_{2}, \mathrm{COOH}, \mathrm{CN}$, or $\mathrm{C}_{n} \mathrm{H}_{2 n+1}$ ), either by applying a potential or spontaneously, which gives an aryl radical that binds to the surface along with $\mathrm{N}_{2}$ as a byproduct (Scheme 2.1e). ${ }^{33,34}$ Alkylphosphonic acids have rarely been used for Si-O-P bond formation. ${ }^{35} \mathrm{Si}-\mathrm{S}$ bond formation has been investigated in a few reports by reacting thiols (R-SH) onto $\mathrm{Si}-\mathrm{H}^{36}$ which has recently been expanded to the use of disulfide, diselenide, and ditelluride reagents for $\mathrm{Si}-\mathrm{S}$, Si-Se, and Si-Te bond formation, respectively 
(Scheme 2.1f). ${ }^{37,38}$ Also, direct Si-Ir bond formation has been reported. ${ }^{39}$ Despite all these recent reports on Si-Se/Te/Ir, no applications have been reported yet.

\subsection{Surface passivation}

When the presence of silicon oxide is undesired, the removal of silicon oxide is easily carried out by immersion in $\mathrm{NH}_{4} \mathrm{~F}$ or $\mathrm{HF}$, but the resulting $\mathrm{H}$-terminated surface is not stable in air. To prevent the regrowth of the native oxide, oxide-free monolayers can be used to passivate the surface. Passivation effects can be divided into two subtypes, i.e., chemical and electrical passivation. Chemical passivation includes resistance against oxidation, whereas electrical passivation implies the reduced surface recombination of charge carriers, which is reflected by lower surface recombination velocities and longer charge carrier lifetimes.

The chemical passivation effect is mostly studied by prolonged exposure to air or water. For saturated alkyl chains on silicon nanowires, the highest resistance against oxidation (>300 $\mathrm{h}$ in air) was observed for the shortest $\left(C_{1}\right)$ monolayers due to their high surface coverage. ${ }^{40,41}$ Assad et al. functionalized silicon nanowires by the chlorination/alkylation route to obtain methyl $\left(-\mathrm{CH}_{3}\right)$, propenyl $\left(-\mathrm{CH}=\mathrm{CH}-\mathrm{CH}_{3}\right)$, or propynyl $\left(-\mathrm{C} \equiv \mathrm{C}-\mathrm{CH}_{3}\right)$ monolayers in almost full coverage. ${ }^{42} \mathrm{X}$-ray photoelectron spectroscopy (XPS) showed that the propenyl monolayers were the most resistant to oxidation during exposure to ambient air (Figure 2.1). For these samples, the oxidation started after $\sim 100 \mathrm{~h}$, increased to 0.15 monolayer of oxide after $\sim 150 \mathrm{~h}$, and was then stable until $\sim 700 \mathrm{~h}$. This higher stability, compared to the methyl and propynyl monolayers, was attributed to the favorable $\pi-\pi$ interactions between the molecules. Ciampi et al. studied the chemical passivation effect of 1,8-nonadiyne monolayers in aqueous environments. ${ }^{43}$ After $\sim 200$ cycles of cyclic voltammetry in an aqueous solution, no oxidation could be detected by XPS $\left(<0.07 \mathrm{SiO}_{x}\right.$ monolayer $)$. Again, the $\pi-\pi$ interactions between the alkyne head moieties resulted in a stabilizing factor, which was disrupted when diluting the monolayer with 1-heptyne molecules. Different headgroups than methyl could be used as well. For example, fluorinated 1-hexadecyne-derived monolayers on silicon nanowires showed a proper chemical passivation effect, since the contact angle hardly changed during exposure to acidic $(\mathrm{pH} 3)$ or basic solutions $(\mathrm{pH} 11)$ for 1 week. ${ }^{44}$ Next to the beneficial passivation effect observed for unsaturated end groups, these moieties allow for secondary functionalization. Depending on the desired application, catalysts, redoxactive moieties, or biomolecules can be coupled onto the surface..$^{42,43,45}$ 


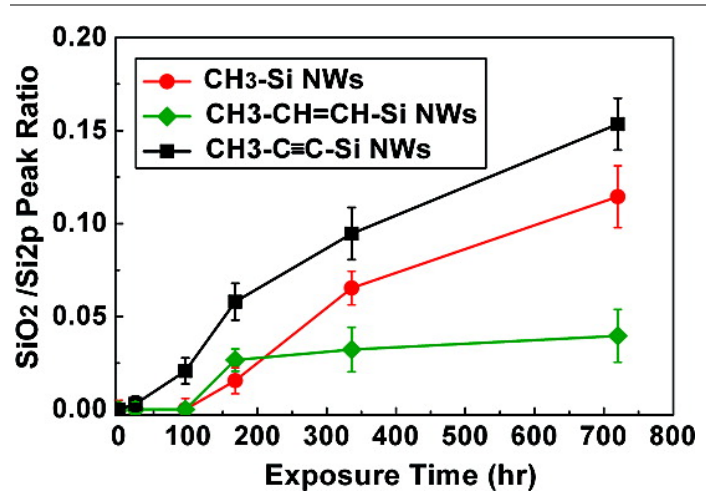

Figure 2.1. Ratio of the $\mathrm{SiO}_{2}$ to $\mathrm{Si} 2 \mathrm{p}$ peak areas from XPS for silicon nanowires with different monolayers, exposed to air for prolonged periods. Reproduced with permission. ${ }^{42}$ Copyright 2008, American Chemical Society.

The electrical passivation effect is often confirmed by a high minority charge carrier lifetime and a low surface recombination rate. ${ }^{46}$ Also in this case, a high surface coverage is beneficial, as shown by a surface recombination velocity $<30 \mathrm{~cm} / \mathrm{s}$ for methyl monolayers compared to $<60 \mathrm{~cm} / \mathrm{s}$ for other linear alkyl chains and $<80 \mathrm{~cm} / \mathrm{s}$ for bulky alkyl groups. ${ }^{47,48}$ Alderman et al. used Kelvin probe measurements to study the electrical passivation of several monolayers, from methyl to butyl, made by the chlorination/alkylation route. ${ }^{49} \mathrm{On} \mathrm{Si}-\mathrm{H}$ and $\mathrm{Si}-\mathrm{Cl}$ surfaces, the minority carrier recombination lifetime was extremely low as expected $(<10 \mu \mathrm{s})$, which is equal to a surface recombination velocity of $>2700 \mathrm{~cm} / \mathrm{s}$. A better electrical passivation, i.e., a higher minority charge carrier lifetime (200-250 $\mu \mathrm{s})$, was observed for methyl, butyl, and tert-butyl monolayers due to a higher surface coverage and a lower number of surface states. The recombination lifetime was measured during storage in air (Figure 2.2) and decreased to about $50 \mu \mathrm{s}$ after $600 \mathrm{~h}$. After 500 days, the values decreased to 10-15 $\mu \mathrm{s}$.

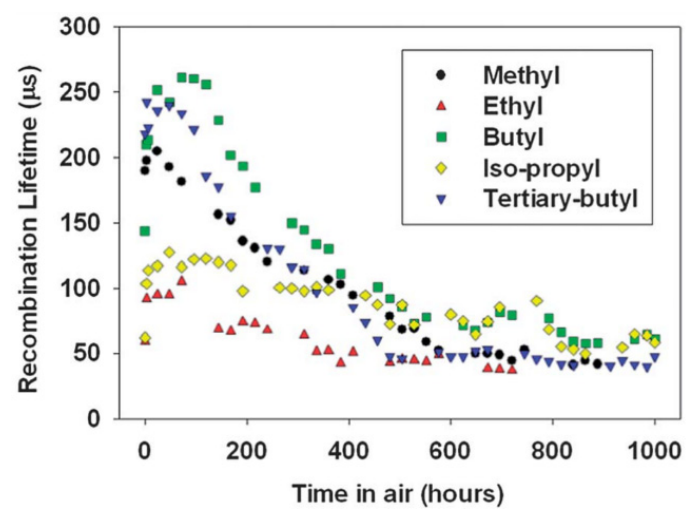

Figure 2.2. The recombination lifetime of minority charge carriers for monolayer-functionalized silicon substrates during exposure to air. Reproduced with permission. ${ }^{49}$ Copyright 2013, The Royal Society of Chemistry. 
The reaction route does influence the effectiveness of passivation effects, which is mainly reflected by differences in surface coverage. ${ }^{50-52}$ Yaffe et al. reacted 1-alcohols onto $\mathrm{Si}-\mathrm{H}$, which led to $\mathrm{Si}-\mathrm{O}-\mathrm{CH}_{2}-\mathrm{R}$ bonds by heating at $80^{\circ} \mathrm{C}$ (nucleophilic substitution) or $\mathrm{Si}-\mathrm{CH}(\mathrm{OH})-\mathrm{R}$ by UV irradiation (radical chain reaction). ${ }^{53}$ The nucleophilic substitution reaction resulted in a better electrical passivation, as observed by less surface band bending and an extended lifetime of minority carriers. In contrast, the reaction mediated by UV light gave more densely packed monolayers and thus showed a higher chemical passivation effect. This trade-off could be balanced by reacting the 1-alcohols at room temperature under UV irradiation. Comparably, Webb et al. compared monolayers made by chlorination/alkylation, Lewis acid catalysis, or anodization of $\mathrm{CH}_{3} \mathrm{Mgl} .{ }^{54}$ The chlorination/alkylation route showed the best passivation properties of this series, with surface recombination velocities of $<200 \mathrm{~cm} / \mathrm{s}$ after $24 \mathrm{~h}$ air exposure. On the other hand, the Lewis acid-based route resulted in surfaces with high recombination values $(>1200 \mathrm{~cm} / \mathrm{s})$, which oxidized as quickly as $\mathrm{Si}-\mathrm{H}$ surfaces. The anodization route gave stable but high recombination rates $(460 \mathrm{~cm} / \mathrm{s})$, but also suffered from extensive oxidation in air.

Passivation layers are attractive for use in solar applications, for example. ${ }^{55,56}$ Solar cells are often 3D structured to improve the absorption of light, but the concomitant increase in surface area also introduces more dangling bonds at the surface. These surface defects create undesired recombination sites for electron-hole pairs, which lowers the solar cell output. ${ }^{57}$ Applying a monolayer as electrical passivation layer reduces this negative effect of dangling bonds. Zhang et al. investigated the use of oxide-free monolayers to electrically passivate a silicon/polymer hybrid solar cell. ${ }^{58} \mathrm{~A}$ methyl/allyl monolayer was created on a silicon nanowire array, after which a poly(3,4-ethylenedioxythiophene)/ poly(styrenesulfonate) (PEDOT:PSS) film was deposited to function as a Schottky diode. The total power conversion efficiency was equal to $9.4 \%, 9.7 \%$, and $10.2 \%$ when using allyl, methyl, or methyl/allyl monolayers, respectively. This trend originated from an increasing surface coverage, since any uncovered $\mathrm{Si}-\mathrm{H}$ sites act as a recombination center. This was also reflected by a trend in the density of trap states for the three different monolayers (Figure 2.3). Accordingly, the charge carrier lifetime increased from 17 to 25 and $29 \mu \mathrm{s}$ for allyl, methyl, and methyl/allyl monolayers, respectively. For solar-to-fuel applications, the use of a passivation layer can be combined with catalyst immobilization. For example, a nickel bisdiphosphine-based hydrogenase mimic was immobilized as a hydrogen evolution catalyst onto an oxide-free amine-terminated monolayer on silicon substrates. ${ }^{59}$ 


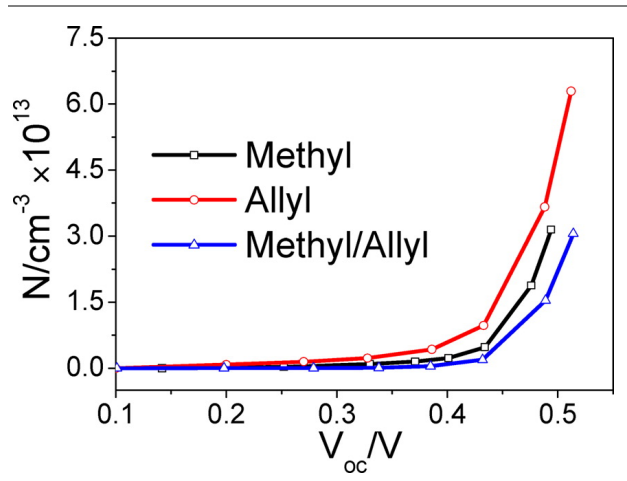

Figure 2.3. Number of density trap states $(N)$ versus the open circuit potential $\left(V_{o c}\right)$ of silicon nanowires/PEDOT:PSS devices with different passivation monolayers. Reproduced with permission. ${ }^{58}$ Copyright 2013, American Chemical Society.

At the same time, chemical passivation is required to avoid oxidation of solar cells in air or photoelectrochemical cells in, mostly acidic or alkaline, aqueous environments. Bashouti et al. exposed both silicon nanowire arrays and planar silicon substrates functionalized with a methyl monolayer to air for 45 days and found a higher oxidation resistance for the silicon nanowire arrays. ${ }^{56}$ This difference was at least a factor of two and was attributed to stronger $\mathrm{Si}-\mathrm{C}$ bonds on the nanowire samples. Shen et al. studied methyl-terminated silicon nanowire arrays as an electrode in a photoelectrochemical cell. ${ }^{60}$ When using these cells in an ionic liquid as the electrolyte, only a little surface oxidation was observed. The stability in water was, however, very poor due to corrosion of the silicon surface within $4 \mathrm{~h}$.

\subsection{Electronics}

The miniaturizing trend in electronic devices puts stringent requirements on the fabrication of silicon-based devices. Molecular monolayers are suitable for adding functionalities at these small scales. Oxide-free layers are often strictly necessary, since silicon oxide functions as an insulating layer and thus prevents the transfer of charges from/to the surface. Silicon substrate doping and electrical sensors are discussed separately in Sections 2.5 and 2.8, respectively.

Extensive literature is available on the coupling of electroactive moieties onto oxide-free silicon substrates, as reviewed recently. ${ }^{61}$ Such moieties can have two stable redox states, such as ferrocene ${ }^{62,63}$ and quinones, ${ }^{64}$ or more than two redox states, such as metalcomplexed porphyrins, ${ }^{62,65}$ tetrathiafulvalene (TTF), ${ }^{66,67}$ and fullerene $\left(C_{60}\right) .^{68}$ These systems are commonly used as model systems to investigate the charge transfer at surfaces, for example to study the influence of the linker length. ${ }^{62,63}$ Ferrocene is frequently studied because of its proper electrochemical characteristics, such as fast electron transfer, low oxidation potential, and stability of both the neutral ferrocene and 
oxidized ferrocenium cation species. ${ }^{61}$ Nonetheless, molecules with multiple electron transfer processes are also gaining more interest because of their potential for high storage memory devices and more sophisticated logic gates.

Molecular monolayers are commonly used in metal-semiconductor junctions, in order to saturate the dangling bonds and avoid direct contact between the metal and semiconductor, for example as a gate insulator. ${ }^{69,70}$ This results in a metal-insulatorsemiconductor (MIS) structure, where a drop of $\mathrm{Hg}$ is often used as a metal contact on top of the monolayer, and the current across the monolayer is measured. The monolayers can be based onto either majority carriers (metal-semiconductor junctions) or minority carriers (behaving like $p$-n junctions). ${ }^{71}$ The MIS junctions that are used for doping applications are described in Section 2.5, whereas the other reports are treated in this section.

Changes in the monolayer, e.g., variations in the surface bond, ${ }^{72,73}$ the terminating group, ${ }^{74-77}$ or molecular length, ${ }^{70,77}$ are of large influence on MIS junctions. In general, the junction characteristics improve at higher monolayer quality, which depends on the packing density, the coverage, and the number of defects of the monolayer. ${ }^{78}$ Changing the monolayer headgroup from $-\mathrm{Br}$ to $-\mathrm{OH}$ strengthened the interactions between the terminal groups, which increased the packing density of the monolayer. ${ }^{74}$ This led to a better ideality factor for the - $\mathrm{OH}$ monolayer, which equaled to 1.30, whereas 1.85 and 1.50 were found for $-\mathrm{Br}$ and $-\mathrm{CH}_{3}$ terminated monolayers, respectively. The lower ideality factor indicates a more homogeneous junction. Faber et al. studied MIS junctions based on several alkyl lengths. ${ }^{70}$ The $J-V$ data were characteristic of Schottky diodes and showed the insulating properties of the molecular monolayers (Figure 2.4). For example, the $\mathrm{C}_{16}$ monolayer $(1.78 \pm 0.02 \mathrm{~nm})$ was thinner than the $\mathrm{SiO}_{2}$ insulator $(1.99 \pm 0.06 \mathrm{~nm})$ but showed lower currents and thus better insulating properties. The current density could be tuned by changing the length of the 1-alkene used, since the insulating properties increased for longer alkyl lengths. This corresponds to an exponential increase in series resistance from $0.49 \Omega \cdot \mathrm{cm}^{2}$ for $C_{10}$ layers to $22.84 \Omega \cdot \mathrm{cm}^{2}$ for $C_{22}$ alkyl chains.

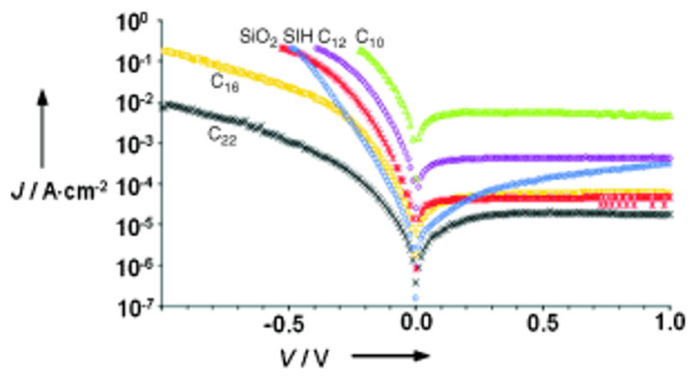

Figure 2.4. J-V characteristics for several alkyl monolayers on p-type $\mathrm{Si}$, including $\mathrm{Si}-\mathrm{H}$ and $\mathrm{SiO}_{2}$ as references. Reproduced with permission. ${ }^{70}$ Copyright 2005, Wiley-VCH. 
Redox-active molecules are investigated for application in memory devices, where oxidation results in charge storage in the monolayer and reduction erases the stored charge. The molecules used should thus be redox-active, easily switchable, and stable for numerous switching cycles. Porphyrin-based molecules, for example, do fulfill these requirements, as they are stable at high temperature $\left(400^{\circ} \mathrm{C}\right)$ for $\sim 1 \mathrm{~h}$ under inert atmosphere. ${ }^{79}$ Moreover, $10^{12}$ switching cycles can be performed without degradation. Also ferrocene has been studied for storage devices in which the two redox states constitute two memory states. ${ }^{61}$ The potential to oxidize ferrocene is $<1.0 \mathrm{~V}$, which allows charge storage with low power consumption. The surface coverage of ferrocene monolayers typically equals $2-5 \times 10^{-10} \mathrm{~mol} / \mathrm{cm}^{2}$, which allows relatively high charge densities $\left(20-50 \mu \mathrm{C} / \mathrm{cm}^{2}\right)$ compared to $\mathrm{Si} / \mathrm{SiO}_{2}$ capacitors conventionally used in dynamic random access memory devices $\left(5-10 \mu \mathrm{C} / \mathrm{cm}^{2}\right) .{ }^{80}$

Fabre et al. studied micropatterned ferrocene-functionalized areas as light-activated memory cells with capacitance performance. ${ }^{80} \mathrm{Si}-\mathrm{H}$ substrates were functionalized with an acid fluoride-terminated monolayer by thermal hydrosilylation, after which an aminefunctionalized ferrocene derivative was microcontact printed onto this monolayer. In this way, square patterns of $5 \times 5 \mu \mathrm{m}^{2}$ with ferrocene molecules were created, and the areas in between were backfilled using butylamine. Under white light illumination, these monolayers showed a maximum capacitance of $120-140 \mu \mathrm{F} / \mathrm{cm}^{2}$ at $50 \mathrm{~Hz}$, which is a significant improvement compared to maximally tens of $\mu \mathrm{F} / \mathrm{cm}^{2}$ for conventional devices. The electrodes could be switched between the ON and OFF states by light illumination (Figure 2.5), and only a 10\% decrease in capacitance density was observed after 1000 ON/OFF cycles. The devices based on $5 \times 5 \mu \mathrm{m}^{2}$ patterns performed better than those with $10 \times 10$ or $20 \times 20 \mu \mathrm{m}^{2}$ regions, which is beneficial for miniaturization. ${ }^{81} \mathrm{Li}$ et al. extended the use of ferrocene for memory devices by adding porphyrin moieties. ${ }^{82}$ Because of the separated potentials of these two molecules, the mixed monolayers could be used as a four-state memory element.

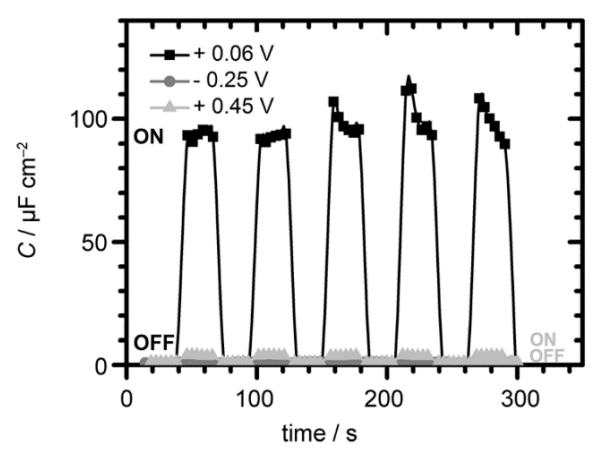

Figure 2.5. Capacitance density of micropatterned ferrocene-terminated silicon surfaces measured at $50 \mathrm{~Hz}$ at different applied potentials, under white light illumination (ON state) or in the dark (OFF). Reproduced with permission. ${ }^{80}$ Copyright 2013, Wiley-VCH. 
Redox-terminated surfaces can also be used for binary logic gates when using the electrical voltage as an input function. Then, a high capacitance density is desired, which should remain constant over many ON/OFF switching cycles. An AND gate was constructed for a ferrocene-terminated monolayer based on a light-induced switch in capacitance, where the two inputs consisted of the illumination level and the applied potential. ${ }^{80}$ The output signal was based on the capacitance measured at $50 \mathrm{~Hz}$, which could be set at " 0 " when the capacitance was $<2 \mu \mathrm{F} / \mathrm{cm}^{2}$ and at " 1 " when the capacitance was $>80 \mu \mathrm{F} / \mathrm{cm}^{2}$. Output " 1 " was only achieved when both the surface was illuminated and the potential was set at the required value $(0.06 \mathrm{~V}$, close to the formal potential of the ferrocene/ferrocenium redox couple). All the other combinations yielded an output signal " 0 ", as required for an AND gate.

\subsection{Doping}

Silicon devices often require doping, i.e., the addition of foreign atoms into the silicon lattice to create an excess of holes ( $p$-type) or electrons ( $n$-type), to modulate its electrical properties or create a p-n junction. The majority of doping methods is based on volume doping, such as ion implantation or chemical vapor deposition. Downscaling of devices, however, requires lower amounts of dopant atoms. Oxide-free monolayers are perfectly suitable for this purpose, in a process called monolayer doping (MLD, Figure 2.6), as has been reviewed recently. ${ }^{83,84}$ In the MLD process, a monolayer of dopant-rich molecules is assembled onto the surface by thermal hydrosilylation ( $\mathrm{Si}-\mathrm{C}$ bonds), after which a capping layer (silicon oxide) is added to prevent loss of the organic monolayer during heating. A short heating step, i.e., rapid thermal annealing (RTA), breaks up the molecular structure and diffuses the dopants into the silicon substrate. After removal of the capping layer, a shallow junction ( $<100 \mathrm{~nm}$ ) is achieved without causing crystal damage. MLD results in uniform junctions, since it is based on the self-limiting monolayer formation. This process has been pioneered by Ho et al., who studied p-type and n-type doping by monolayer formation of allylboronic acid pinacol ester and diethyl 1-propylphosphonate, respectively. ${ }^{85}$ After RTA for $5 \mathrm{~s}$ at $900-1050{ }^{\circ} \mathrm{C}$, secondary ion mass spectrometry (SIMS) measurements showed a junction depth of $<50 \mathrm{~nm}$ and surface concentrations of $\sim 5 \times 10^{20}$ atoms $/ \mathrm{cm}^{3}$ and $\sim 1 \times 10^{22}$ atoms $/ \mathrm{cm}^{3}$ for boron and phosphorus, respectively. The use of an oxide-free monolayer is needed to make the dopant diffusion more efficient. $^{85}$ 


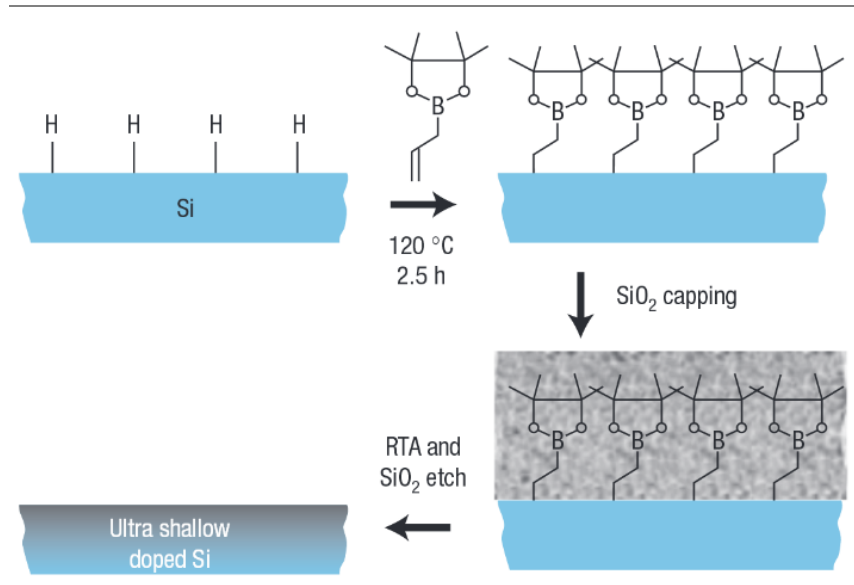

Figure 2.6. Schematic representation of the monolayer doping (MLD) process on silicon substrates. Reproduced with permission. ${ }^{85}$ Copyright 2008, Nature Publishing Group.

The MLD method allows tunability of the doping concentration and doping dose tailored to the desired device. The junction depth can be regulated by the RTA step and temperature, which results in shallow junctions even down to ultrashallow sub-5 $\mathrm{nm}$ junctions. ${ }^{86}$ The total doping dose can be tuned downwards by the use of a bulkier precursor $^{85}$ or by mixing in alkene molecules without dopant atoms. ${ }^{87}$ The latter technique tuned the surface concentrations from $2 \times 10^{19}$ atoms $/ \mathrm{cm}^{3}$ for $100 \%$ dopant molecules (boron and phosphorus) down to $4 \times 10^{18}$ atoms $/ \mathrm{cm}^{3}$ (boron) and $2 \times 10^{18}$ atoms $/ \mathrm{cm}^{3}$ (phosphorus) for $0.1 \%$ dopant adsorbates, i.e., a variation over one order of magnitude. Conversely, the total amount of doping can be regulated upwards by the use of dopant-rich molecules, for example using carborane molecules that have 10 boron atoms per molecule, which resulted in surface concentrations of $\sim 5 \times 10^{19}$ atoms $/ \mathrm{cm}^{3}{ }^{88}$ The MLD method has been expanded to the use of arsenic atoms as n-type dopants using triallylarsine, ${ }^{89}$ methylarsenic acid, ${ }^{90}$ or arsenic azide by click chemistry. ${ }^{91}$ Recently, the use of nitrogen-containing precursor molecules has also been reported, ${ }^{92}$ since nitrogen atoms have a lower thermal diffusion coefficient than phosphorus or boron. This allows for a more accurate annealing process as longer annealing times, i.e., $1050{ }^{\circ} \mathrm{C}$ for $2 \mathrm{~min}$, can be used while still obtaining shallow junctions $(<50 \mathrm{~nm})$. From the in-diffused atoms, however, only $0.5 \%$ was electrically active, which makes this route unacceptable for device applications. Alternatively, alkylphosphonic acids can be used for n-type doping, which form monolayers through a Si-O-P bond. ${ }^{35}$ These bonds are stable up to high temperatures, so the need for a capping layer is avoided. Alkyl chains were released at $500{ }^{\circ} \mathrm{C}$, i.e., before the dopant diffusion occurs, which avoids any carbon contamination.

The MLD process is compatible with 3D nanostructure doping, which makes MLD suitable for doping electronic devices, for example, fin field-effect transistors (FinFETs) ${ }^{93,94}$ For 
example, Ho et al. applied MLD to dope Si nanowires, of which a two-terminal device was constructed. ${ }^{85}$ The doping of the nanowires lowered their resistance at $1 \mathrm{~V}$ from $\sim 100 \mathrm{G} \Omega$ for undoped nanowires to $\sim 2 \mathrm{M} \Omega$ for nanowires doped by n-type MLD. The MLD process was also found to be suitable for fabricating FETs from Si-on-insulator substrates. Photolithography was used to make heavily doped p-type Si regions underneath the Si-metal source/drain contacts only, whereas the channel remained intrinsically doped. This changed the FET performance from a $\sim 0.1 \mu \mathrm{A}$ source-drain current at $0.5 \mathrm{~V}$ for a device with undoped contacts to more than $1.0 \mu \mathrm{A}$ for a doped device. ${ }^{85}$ If local functionalization is required on a smaller scale, nanoimprint lithography can be used to perform MLD at a nanoscale resolution. ${ }^{95}$ This makes MLD a technique that is compatible with the downscaling trend in semiconductor industry.

As another example of device fabrication, O'Connell et al. fabricated Si nanowires with a diameter from 20 to $1000 \mathrm{~nm}$, which were doped with arsenic atoms by MLD. ${ }^{89}$ Even the $20 \mathrm{~nm}$ devices showed a proper current conduction, which indicates that the MLD process can be applied to small feature sizes as well. The resistivity of the nanowires was decreased by five orders of magnitude for nanowires with a width $>40 \mathrm{~nm}$ and by seven orders of magnitude for smaller nanowires.

A variation of the MLD process includes monolayer contact doping (MLCD, Figure 2.7), in which the dopant-containing monolayer is formed on an external substrate. This dummy substrate is brought into contact with the target substrate, after which the stack of substrates is annealed. The formation of a monolayer and a capping layer on the target substrate is avoided by this strategy, which can thus be used when the target substrate is not compatible with HF etching, which is usually required to remove the native oxide and the capping layer. Hazut et al. first employed this technique using phosphine oxide monolayers on intrinsic dummy substrates with native oxide. ${ }^{96}$ In a nanowire device, n-type doped nanowires were obtained by MLCD, which resulted in a highly uniform dopant distribution along the nanowire length (Figure 2.7). The starting intrinsic nanowires performed as an inactive source-drain channel with a resistance of $\sim 20 \mathrm{G} \Omega$ at $2 \mathrm{~V}$, which was lowered to $\sim 20 \mathrm{k} \Omega$ after MLCD. MLCD was thus confirmed to be a successful alternative to MLD. The technique was also used to create parallel $p$-i-n junctions inside nanowires. ${ }^{97}$ Here, Hazut et al. applied MLCD to undoped nanowires by sandwiching the nanowires between two dummy substrates, including one boroncontaining (phenylboronic acid) and one phosphorus-containing (tetraethyl methylenediphosphonate) donor substrate. The junction formation was confirmed by scanning tunneling microscopy measurements in combination with scanning tunneling spectroscopy. 

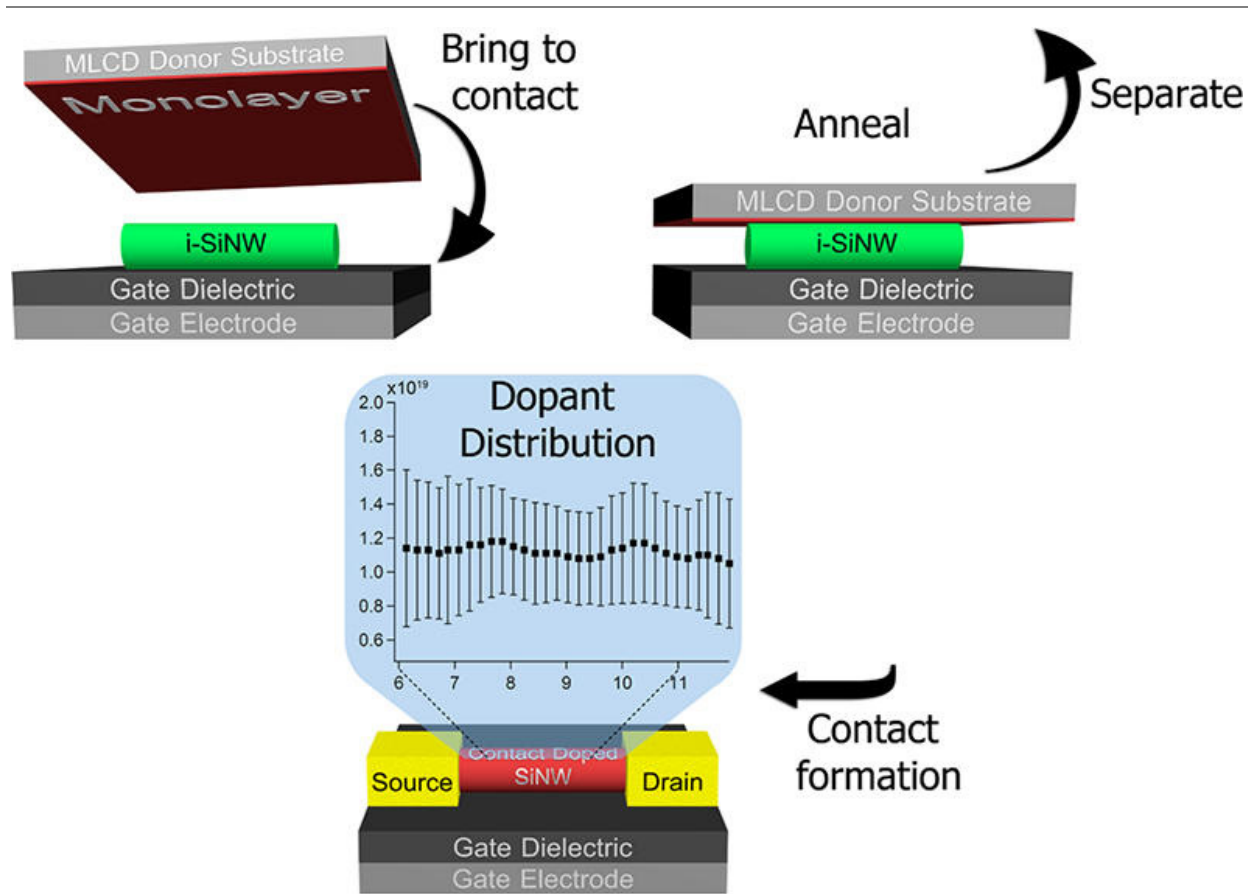

Figure 2.7. Schematic representation of the monolayer contact doping (MLCD) process on silicon nanowires. Reproduced with permission..$^{96}$ Copyright 2012, American Chemical Society.

Whereas the applications of MLD and MLCD described above focused on electronic devices, Puglisi et al. have used MLD for Si nanowire-based solar cells. ${ }^{98}$ Here, doping can be used to create $p-n$ junctions for solar light-induced charge separation. Vertical Si nanowire arrays with a nanowire diameter of $<70 \mathrm{~nm}$ and a length of about $500 \mathrm{~nm}$ were grown by metal-catalyzed chemical vapor deposition. After removal of the gold catalyst, the $\mathrm{H}$-terminated $\mathrm{Si}$ nanowires were dip-coated in a solution of diethyl 1-propylphosphonate. Thermal annealing at 900 or $950{ }^{\circ} \mathrm{C}$ for $500 \mathrm{~s}$ led to a phosphorus concentration of $10^{19}$ atoms $/ \mathrm{cm}^{3}$ and doping of the entire nanowires. The substrates were measured as solar cells, of which a rectifying junction behavior was observed as expected. Electrical characterization showed an increase in the fill factor from 10 to 35\% for the MLD solar cells compared to planar control samples treated equally, although the open-circuit voltage decreased from 0.42 to $0.27 \mathrm{~V}$. The solar cell performance can be increased even further when using 3D micropillars/nanowires with a radial $p-n$ junction, since the larger surface-to-volume ratio and larger junction area enhance the efficiency. The use of MLD to create radial junctions was also reported in solar cells based on nanohole arrays, ${ }^{99,100}$ where the doping was also found to be conformal in the vertical direction. 
Surface transfer doping has been used to obtain p-n junction behavior without physically implementing foreign atoms into the silicon lattice. In this technique, the coupling of molecules onto the surface is used to tune the electrical properties of silicon, so that the charge transfer between the semiconductor surface and the monolayer can be used to locally manipulate the silicon conductivity. ${ }^{101,102}$ The changes depend on the electrondonating ability, work function, and/or dipole moment of the grafted molecules. ${ }^{103}$ To study this effect, Zhang et al. coupled an azobenzene-containing molecule to a silicon surface by hydrosilylation, after which the dipole moment of the azobenzene moiety was changed by switching between UV (to create the cis configuration) and visible light (to create the trans state) illumination. ${ }^{104}$ This changed the electrical properties of the silicon surface, as the conductivity was higher when the molecules were in the trans state. Whereas surface transfer doping is already effective for adsorbed molecules, ${ }^{105}$ covalent monolayer formation is beneficial for allowing close contact between the dopants and the substrate and for avoiding dopant removal. When the adsorbate molecules are electron-withdrawing, the surface behaves as a p-type semiconductor, whereas electrondonating molecules result in $\mathrm{n}$-type conductivity. For example, the binding of terpyridine molecules onto silicon nanowires resulted in a shift of the Fermi level towards the conduction band, which is similar to n-type doping. ${ }^{106}$

The field of surface transfer doping on silicon is still in its infancy, especially regarding the study of possible applications. Since surface transfer doping is compatible with 3D nanostructures, a few reports are available on the use of molecular monolayers on FETs. ${ }^{106-108}$ For example, He et al. used electrografting to create monolayers that influenced the conducting properties (drain current and threshold voltage) of the channel region of metal-oxide-semiconductor FETs (MOSFETs). ${ }^{109}$ Molecules with different electron-donating abilities were used, i.e., $\mathrm{N}_{2}{ }^{+}$-phenyl- $\mathrm{X}$ with $\mathrm{X}=\mathrm{N}\left(\mathrm{CH}_{3}\right)_{2}, \mathrm{NH}_{2}, \mathrm{NO}_{2}$, or a $\mathrm{Mo}_{6}$ oxide cluster (Figure 2.8a). These molecules were grafted onto $\mathrm{H}$-terminated silicon by exposing the sample to a $0.5 \mathrm{mM}$ solution of the diazonium salts, resulting in arylsilicon bonds (Figure 2.8b). For the electron donating molecules 1-3, molecular grafting led to a lower drain current with the highest decrease for molecule 1 and a more negative threshold voltage. The opposite effect was observed when grafting electron-withdrawing molecule 4. The channel conductance was reduced by grafting 1-3 and increased by 4, which scaled directly with the relative electron-donating abilities of the molecules. Similarly, Bashouti et al. modulated n-type Si nanowires in FETs by monolayers with 1,3-dioxan-2-ethyl, butyl, allyl, and propylalcohol moieties by the chlorination/alkylation route. ${ }^{107}$ Here, the channel conductance showed a trend in the order Si-propylalcohol < $\mathrm{Si}_{-} \mathrm{SiO}_{2}<\mathrm{Si}$-allyl $<\mathrm{Si}$-butyl $<\mathrm{Si}$-1,3-dioxan-2-ethyl, which was correlated to a decreasing change in work function. 


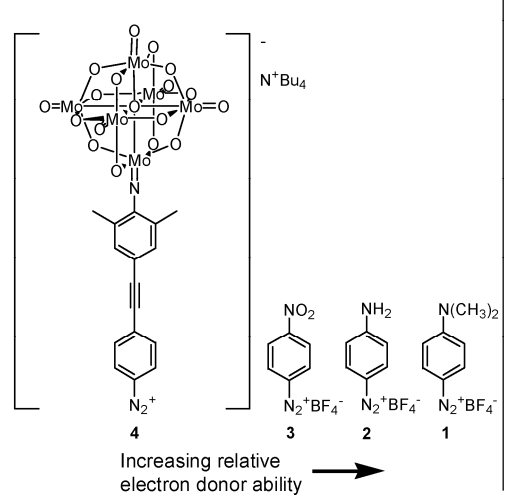

b
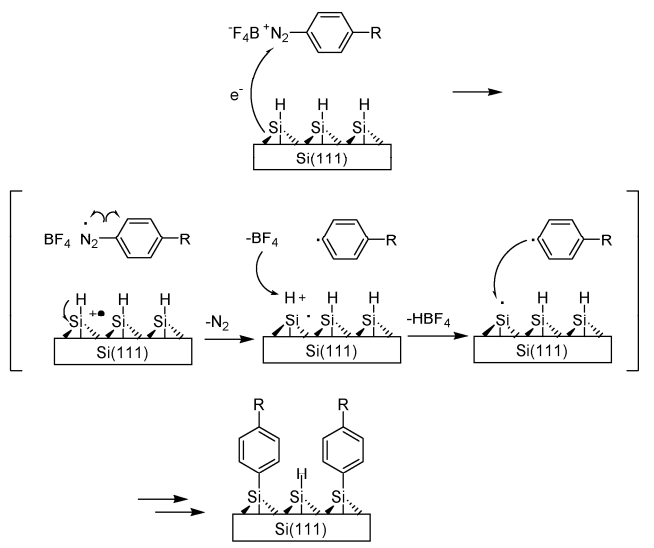

Figure 2.8. a) Chemical structures of the diazonium salts used for electrografting on the channel of a MOSFET. b) The mechanism of the electrografting reaction onto H-terminated Si. Reproduced with permission. ${ }^{109}$ Copyright 2006, American Chemical Society.

Similar to surface transfer doping, metal-insulator-semiconductor (MIS) junctions can result in inversion of the doping carriers, i.e., the carrier type that is dominant at the surface is opposite from the bulk carrier type. ${ }^{71}$ The inverted region then behaves like a $p-n$ junction. ${ }^{110}$ This is reflected in asymmetric $J-V$ characteristics with semiconductorlimited transport at reverse bias up to low forward bias and monolayer thicknessdependent transport at high forward bias. Har-Lavan et al. studied the electrical properties of hydroquinone/1-alcohol monolayers on $\mathrm{Si}-\mathrm{H}$, which led to a surface with Si-O-C bonds, of which 95\% were methoxy and 5\% were hydroquinone groups. ${ }^{69}$ After contacting the surface by $\mathrm{Hg}$, the junction behavior was measured to be close to the Schottky-Mott model, which represents ideal metal-semiconductor junctions. The molecular surface dipole could be changed by $400 \mathrm{mV}$ by changing the length of the alkyl molecules, which resulted in a near-ideal effective electron affinity. The shortest alcohol, i.e., methanol, resulted in the largest surface dipole. The silicon $\mathrm{n}^{+}$substrate was then inverted, so that the monolayer region behaved like $\mathrm{p}^{+}$type semiconductor and the substrate could be measured as a photovoltaic device (Figure 2.9). The open-circuit voltage, i.e., the cross section at the x-axis, shifted towards higher values for substrates with higher substrate doping concentrations (Figure 2.9a). 

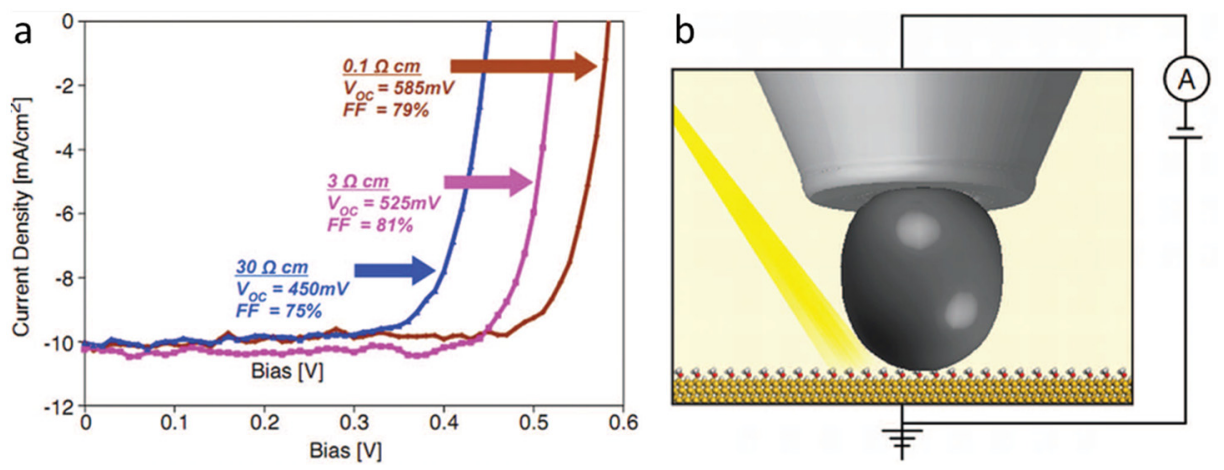

Figure 2.9. a) $J-V$ measurements for $\mathrm{n}-\mathrm{Si}(100)$ substrates with different doping concentrations, with a hydroquinone/methanol monolayer on top, measured under $561 \mathrm{~nm}$ illumination and for comparison adjusted to $J_{p h}=10 \mathrm{~mW} / \mathrm{cm}^{2}$. b) Cartoon of the metal-insulator-semiconductor junction. Reproduced with permission. ${ }^{69}$ Copyright 2012, AIP Publishing.

\subsection{Optics}

For optical or optoelectronic applications, silicon is used in either its porous ${ }^{111}$ or nanoparticle ${ }^{112}$ form, since both possess bright photoluminescence properties at room temperature. These light emission properties can be beneficial for displays, sensing devices, or solar cells. Both silicon configurations, however, suffer from instability and loss of photoluminescence by oxidation. ${ }^{113,114}$ For planar porous silicon, oxide-free monolayers of 1-alkenes, nonconjugated dienes, and aldehydes are the most suited, since monolayers of 1-alkynes or conjugated molecules tend to quench the photoluminescence to large extents. ${ }^{115}$ The reaction route is also of importance, since the use of alkyllithium reagents does result in Si-alkyl and Si-Li surface species, of which the latter is easily hydrolyzed by water and thus results in surface oxidation and a reduced photoluminescence. ${ }^{116,117}$ Similarly, the reaction of 1-alcohols onto Si nanoparticles first quenches the photoluminescence almost completely, which can then be slightly recovered until $10-50 \%$ of the original intensity by a heating step. ${ }^{118}$ Choi et al. studied the influence of different monolayer formation routes on the electrochemiluminescence properties, i.e., luminescence by bias-induced charge injection from a hole/electron source, of porous silicon substrates. ${ }^{119}$ When comparing cathodic/anodic electrografting and Lewis acid-based hydrosilylation, anodic electrografting yielded the brightest emission, but this intensity was only half the value of unfunctionalized $\mathrm{Si}-\mathrm{H}$ surfaces. The emission lifetime was, however, twice as long for the functionalized samples and could be recovered by $10 \mathrm{~s}$ application of a cathodic bias.

On porous silicon substrates, ethyl undecylenate, ${ }^{120} 1,7$-octadiene, ${ }^{115}$ octyl aldehydes, ${ }^{115}$ and 1-dodecene, ${ }^{121}$ amongst others, have been shown to passivate the surface and preserve the photoluminescence properties. Stewart et al. have further explored the 
stability of these photoluminescent surfaces. ${ }^{122}$ To make a distinction between functionalized and unfunctionalized regions, they functionalized porous silicon with 1-alkenes/1-alkynes by local light-promoted hydrosilylation through a photomask. In three steps, different regions of the substrate were functionalized with monolayers of 1-decene, styrene, and 1,5-cyclooctadiene as patterned in their corresponding names (Figure 2.10a). After $15 \mathrm{~s}$ immersion in a boiling $\mathrm{KOH}$ solution $(\mathrm{pH} 12)$, which would normally dissolve micrometer-thick porous silicon layers, UV light irradiation revealed that the patterned areas were still photoluminescent (Figure 2.10b). The untreated background areas were dark and had lost their photoluminescence. Thus, the monolayers stabilized the photoluminescent properties, even when subjected to severe etching conditions by hydroxide ions. This effect appeared to depend on the nature of the monolayer, since the photoluminescence intensity was still at its original value with a monolayer of 1-dodecene, whereas the intensity dropped to $\sim 34 \%$ when adding an aliphatic alkenyl group (a monolayer of 1-octyne) or was even completely quenched by a monolayer of conjugated molecules, such as phenylacetylene (Figure 2.10c).
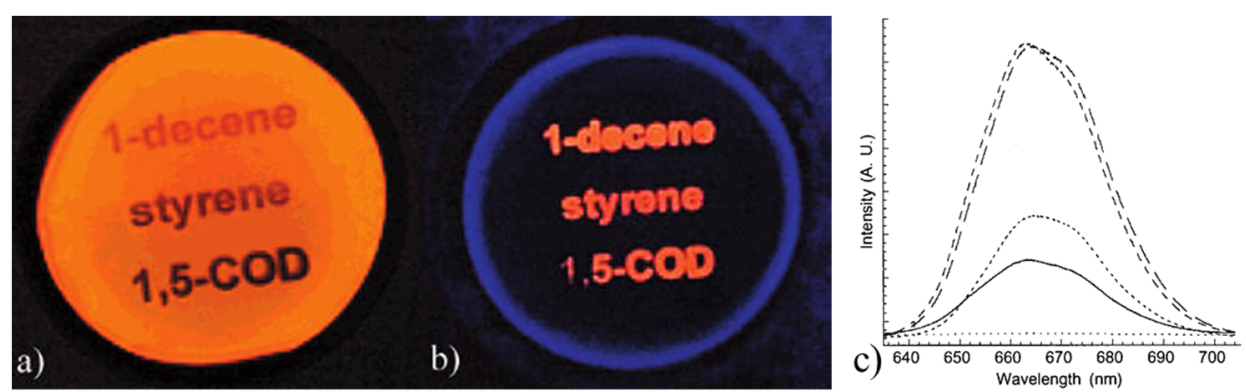

Figure 2.10. a,b) A porous silicon substrate locally functionalized with 1-decene, styrene, and 1,5-cyclooctadiene, as imaged under UV (365 nm) illumination after a) monolayer formation and b) immersion in a boiling $\mathrm{pH} 12 \mathrm{KOH}$ solution. c) The photoluminescence spectra of several monolayers on porous silicon, in order of decreasing intensity: Si-H (--), dodecyl $(--)$, phenethyl $(---)$, octenyl (-), styrenyl (…). Reproduced with permission. ${ }^{122}$ Copyright 2001, American Chemical Society.

Luminescent silicon nanoparticles $(3-6 \mathrm{~nm}$ ) also need surface passivation to prevent oxidation and loss of photoluminescence properties. ${ }^{113}$ Although silane monolayers have been investigated for this purpose, ${ }^{113,123}$ oxide-free monolayers are more suited for surface passivation, especially when aqueous environments are needed. For example, Li et al. applied the thermal hydrosilylation technique to stabilize the photoluminescence properties of silicon quantum dots. ${ }^{113}$ After nanoparticle fabrication, etching in a $\mathrm{HF} / \mathrm{HNO}_{3}$ solution was used to downsize the particles. Although these particles already showed bright visible photoluminescence, the stability was significantly increased when adding a 1-octadecene or undecylenic acid monolayer onto the Si-H surface. These monolayers prevented oxidation of the surface, particle agglomeration, and a blueshift 
of the photoluminescence (Figure 2.11). The 1-octadecene-treated nanoparticles did not show a change in photoluminescence intensity and peak position after 60 days storage in air, whereas the untreated samples showed a decrease in intensity (factor $\sim 24$ ) and a blueshift in peak position within 12 days. This stabilization was attributed to the passivation of the surface, since any surface oxidation was observed by a blueshift. Correspondingly, nanoparticles with a monolayer of undecylenic acid were less stable than those functionalized with 1-octadecene, since the undecylenic acid monolayer was less densely packed.

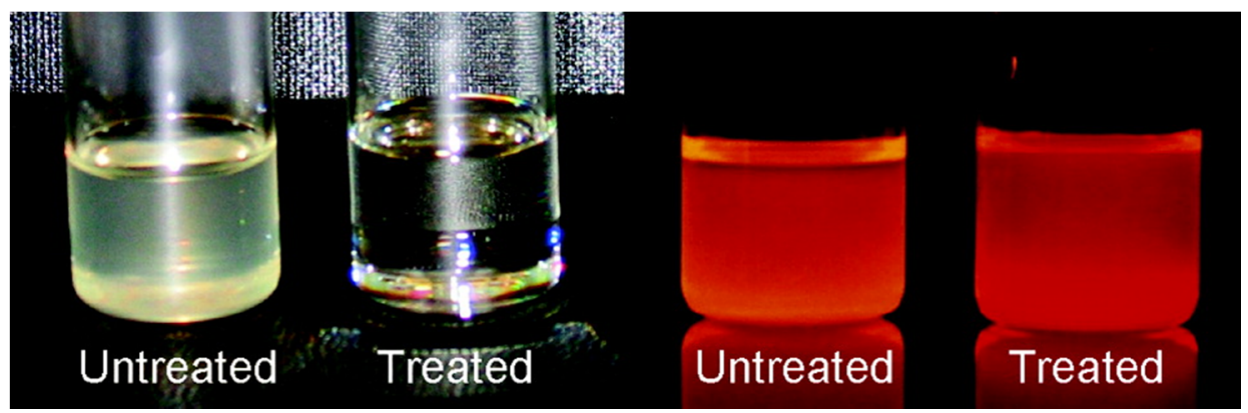

Figure 2.11. Silicon nanoparticles with or without a 1-octadecene monolayer under ambient illumination (left two pictures) or UV illumination (right two pictures). Reproduced with permission. ${ }^{113}$ Copyright 2004, American Chemical Society.

Next to the packing density of the monolayer, the presence of a $\pi$-electron system also influences the luminescence properties of the silicon nanoparticles. ${ }^{124,125}$ The use of $\pi$-conjugated monolayers, e.g., styrene or 1-vinyl naphthalene, not only stabilizes the luminescence properties but also increases the radiation rate compared to 1-decene monolayers. This is opposite to the effect described above for porous silicon substrates, where any conjugated monolayer quenches the photoluminescence. Rosso-Vasic et al. studied the influence of the monolayer length on the luminescence properties. ${ }^{126}$ They synthesized monodisperse silicon nanoparticles $(1.57 \pm 0.21 \mathrm{~nm})$ functionalized with alkyl monolayers by immersing the nanoparticles in a degassed alkene solution with $\mathrm{H}_{2} \mathrm{PtCl}_{6}$ as a catalyst. Infrared spectroscopy and X-ray photoelectron spectroscopy showed no surface oxidation for alkyl monolayers longer than twelve carbon atoms. The surface coverage was about $50 \%$, which is close to the value observed for planar substrates. The fluorescence quantum yield increased for monolayers of longer alkyl chains, i.e., from $13 \%$ for 1 -decene to $23 \%$ for 1 -hexadecene monolayers.

If an optical device is made from a nonporous silicon substrate, monolayers can be used to add luminescent properties by the immobilization of metal complexes. For example, a nickel(II)-based complex was grafted onto Si-H by thermal hydrosilylation, which was confirmed by scanning near-field optical microscopy. ${ }^{127}$ As another option, a multiple- 
step reaction is often used to prevent degradation of the metal complexes during the hydrosilylation reaction, for example for lanthanide complexes. Since the optical properties of these complexes are highly influenced by the geometry of the molecules, a europium(III) complex was coupled indirectly to an N-hydroxysuccinimide (NHS) monolayer by nucleophilic substitution by Condorelli et al. ${ }^{128}$ This multistep process preserved the luminescent properties of the complex.

\subsection{Biomedical devices}

For biological applications, a device is required to be stable in biological fluids or aqueous environments. Si-C linkages are perfectly suited for this purpose, as opposed to silanebased monolayers, for example, of which the Si-O bonds are susceptible to hydrolysis. ${ }^{129}$ Porous silicon degrades in aqueous environments into nontoxic silicic acid within hours to months, depending on its porosity. ${ }^{129}$ The degradation can be advantageous in the case of drug delivery applications, for example, but is undesired for longer term applications. Canham et al. studied the use of oxide-free monolayers to stabilize porous silicon under in vivo conditions, in this case human blood plasma. ${ }^{130} \mathrm{~A}$ Lewis acid-based hydrosilylation route was used to bind 1-dodecyne onto porous silicon, which was then subjected to physiological conditions for several weeks. Whereas the porous film of Si-H control samples was already dissolved within $70 \mathrm{~h}$, the monolayer-functionalized samples hardly changed upon 4 weeks of immersion in simulated body fluid. The oxygen detected along the depth of the sample was just slightly higher for a monolayer sample after 4 weeks under physiological conditions compared to a monolayer sample that had been stored in air for 6 weeks. In addition, Böcking et al. reported the influence of the monolayer coverage on the stability of the porous silicon and its secondary functionalities. ${ }^{131}$ As expected, the higher the surface coverage of the 10-undecenoic acid monolayer, the slower the oxidation and thus dissolution of silicon in aqueous environments. For the samples with the highest monolayer coverage, however, the majority of the carboxylic acid moieties was not available anymore for secondary functionalization by 1-ethyl-3-(3-dimethylaminopropyl)carbodiimide (EDC)/NHS coupling with a tripeptide. This difference was attributed to steric hindrance of the densely packed headgroups. An optimum was found in an intermediate level of alkylation, which showed both a low amount of oxidation and a high functionalization yield.

The immobilization of biomolecules has been a topic of wide interest, e.g., for sensor applications (as described in Section 2.8) or cell studies. Monolayer formation of biomolecules has to be carried out by a mild functionalization technique to avoid deterioration of these mostly labile molecules. For direct coupling onto $\mathrm{Si}-\mathrm{H}$, the mild photochemical route is commonly used, for example to make monolayers of carbohydrates ${ }^{132-134}$ or NHS-biotin combinations. ${ }^{135}$ Alternatively, an indirect route can be used to first establish a densely packed monolayer, onto which the bulkier 
biomolecules are reacted by, for example, click chemistry, ${ }^{136}$ thiol-ene chemistry, ${ }^{137}$ or EDC/NHS coupling. ${ }^{138}$ The latter methods have led to the immobilization of DNA, ${ }^{138-142}$ peptides, ${ }^{143}$ biotin-avidin sequences, ${ }^{144-146}$ antibodies, ${ }^{147}$ or other proteins. ${ }^{129,148,149}$ The desired application then determines the functionality to be used. For example, Qin et al. created an alkyne-functionalized monolayer, protected with a trimethylgermanyl group, which could be reacted subsequently with azide-containing mannose molecules in order to capture E. coli bacteria. ${ }^{136}$ The same platform was used to bind azide-functionalized biotin or mannose onto the surface, which was backfilled with oligo(ethylene glycol) moieties to prevent nonspecific adsorption (Figure 2.12). Protein immobilization was achieved by specific biotin-avidin and mannose-concanavalin A interactions, respectively. The higher the amount of oligo(ethylene glycol) units, the lower the fluorescence intensity of the immobilized proteins, as expected. The optical properties of porous silicon or silicon nanoparticles can also be used for biological applications. The stabilizing effect of the oxide-free monolayer on the photoluminescence (Section 2.6) is then combined with the monolayer's opportunities for secondary functionalization. For example, amino-modified oligonucleotides ${ }^{150}$ or biotin/streptavidin ${ }^{151}$ were coupled onto monolayers with an NHS ester in order to enable the use of Si nanoparticles in biological applications, where both solubility and stability in aqueous environments are required.

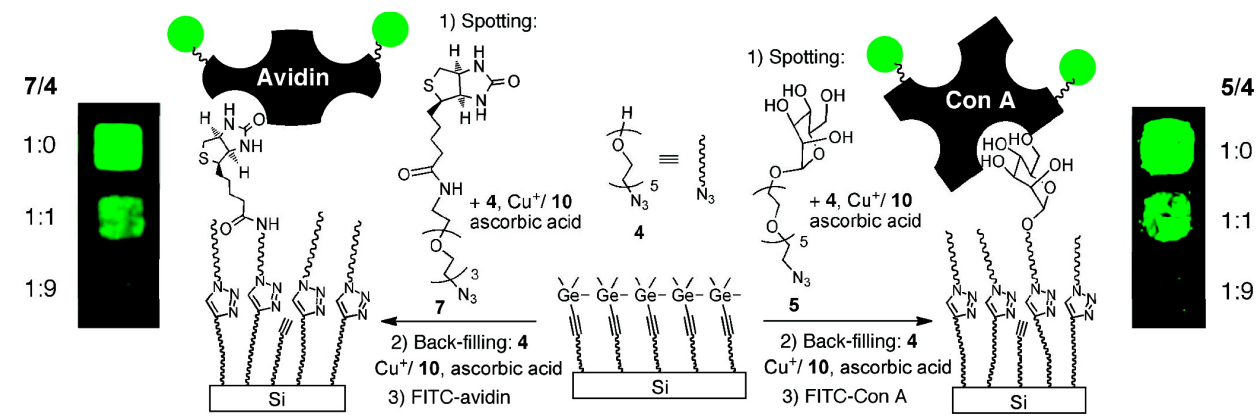

Figure 2.12. Schematic overview a trimethylgermanyl-protected alkyne monolayer with subsequent click chemistry of (left) azide-functionalized biotin for interactions with avidin, and (right) azidefunctionalized mannose for interactions with concanavalin A. Both substrates were backfilled with azide-containing oligo(ethylene glycol) moieties and measured using fluorescence microscopy. Reproduced with permission. ${ }^{136}$ Copyright 2010, American Chemical Society.

Apart from the targeted attachment of (bio)molecules or (bio)compounds described above, antifouling properties could be desired as well. Antifouling is mostly achieved by monolayers that contain oligo(ethylene glycol) moieties. ${ }^{129,152-155}$ Sweetman et al. studied the antifouling properties of Si-C-bound monolayers on porous silicon. ${ }^{129}$ Thermal hydrosilylation was used to bind two alkenes, which were identical apart from a tri(ethylene glycol) group. The oligo(ethylene glycol) group exhibited low-fouling properties towards bovine serum albumin (BSA) but not towards fibronectin (Figure 2.13, 
"adsorbed BSA" or "adsorbed fibronectin"). As a comparison, the fluorescence intensity of covalently bound proteins was added as well, which was consequently higher than that of the adsorbed proteins. They expected the oligo(ethylene glycol) moiety to be too short to prevent adsorption of the bulkier fibronectin, which contains two dimer subunits of $250 \mathrm{kDa}$ compared to $66 \mathrm{kDa}$ for BSA. Flavel et al. observed that the small protein lysozyme (15 kDa) tended to penetrate into the poly(ethylene glycol) monolayer, which made the antifouling properties less effective in repelling this small protein. ${ }^{152}$ Kilian et al. showed that hexa(ethylene glycol) groups not only lead to antifouling properties but also to a higher stability. ${ }^{153}$ Surfaces functionalized with 10-undecylenic acid only withstood exposure to blood plasma at $37^{\circ} \mathrm{C}$ (physiological conditions) for less than $50 \mathrm{~h}$, whereas the samples with hexa(ethylene glycol) had not changed after $72 \mathrm{~h}$.

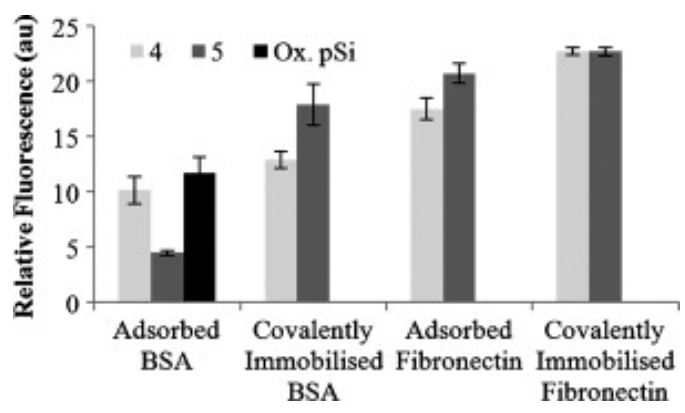

Figure 2.13. Relative fluorescence intensities of adsorbed or covalently bound proteins, i.e., fluorescently labeled BSA or fibronectin, on an alkyl monolayer ("4"), an alkyl monolayer with tri(ethylene glycol) (" 5 "), or unfunctionalized porous silicon ("ox. pSi"). Reproduced with permission. ${ }^{129}$ Copyright 2011, Elsevier.

Porous silicon is an attractive platform for drug release applications, because of the tunability of its pore size and volume, its large surface area, its biodegradability, and its biocompatibility. ${ }^{156,157}$ Oxide-free monolayer functionalization of these surfaces can be of added value, mainly because of the stabilizing effect on porous silicon, as described earlier in this section, and thus a more controlled drug release rate. ${ }^{157}$ The drug can be loaded into the pores of porous silicon ${ }^{158-160}$ or covalently bound to the substrate. ${ }^{156,161}$ As an example of the first type, Anglin et al. created monolayers of 1-dodecene on porous silicon substrates and studied the release of the steroid dexamethasone by a change in optical reflectivity. ${ }^{158}$ The 1-dodecene monolayer prevented, however, loading of the steroid molecules into the pores due to steric hindrance. This was solved by pore widening in the porous silicon preparation step. Release of the steroid was tested in a phosphate-buffered saline solution and turned out to be more controlled for the 1-dodecene-functionalized samples (Figure 2.14). About 90\% of the drug was released over 3 days for the modified samples, whereas the unmodified substrates were already dissolved within $3 \mathrm{~h}$. Alternatively, the drug can be covalently bound to the silicon surface 
in order to control the release by bond breaking. Wu et al. attached the anticancer drug doxorubicin covalently to mesoporous silicon microparticles by a linker of 10-undecenoic acid. ${ }^{161}$ The release was then triggered by oxidation of the Si-Si substrate bonds in water, leading to slow release of the drug. This process was accelerated by adding peroxynitrite as oxidizing species. Similarly, the controlled release was studied for the covalently bound drug daunorubicin, ${ }^{156}$ which is used to treat a specific ocular disease or as an anticancer drug. By degradation of the porous silicon substrate, the drug was released in a linear fashion over 30 days, which is longer than can be obtained by injection of the pure drug.

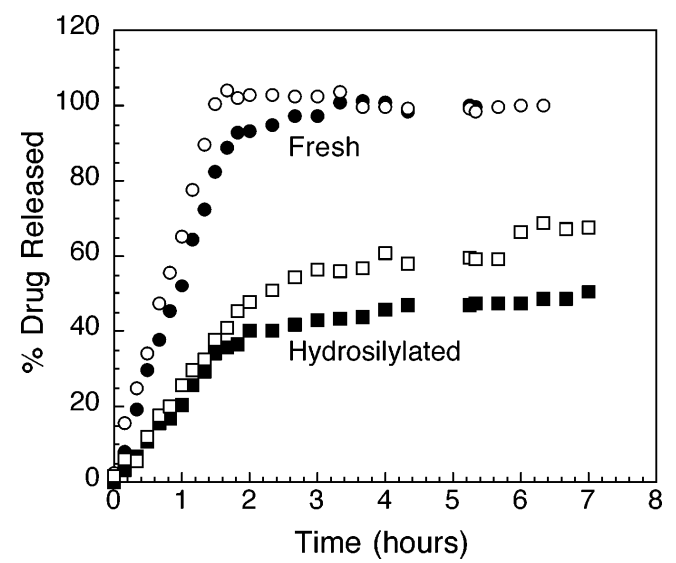

Figure 2.14. Percentage of dexamethasone released as a function of time as measured by UV absorbance in solution. The circles and squares represent unmodified ( $\mathrm{H}$-terminated) and modified (1-dodecene) samples, respectively. The open and solid shapes denote duplicate batches for comparison. Reproduced with permission. ${ }^{158}$ Copyright 2004, American Chemical Society.

\subsection{Sensors}

For chemical sensing applications, an analytical signal is generated as a consequence of the recognition process between the receptor and the analyte. For this purpose, Si-C linkages are beneficial because of the improved signal transfer to the substrate. In biosensing applications, the $\mathrm{Si}-\mathrm{C}$ linkages improve the stability in biological fluids or aqueous environments (see Section 2.7). Sensing devices can be based on a variation in fluorescence, optical, or electrical properties, or a mass change, of which several examples are listed in this section.

Sensors based on supramolecular chemistry are advantageous because of the inherent reversibility of noncovalent interactions, which allows a repeated use of the sensor. For example, Condorelli et al. have studied monolayers of quinoxaline cavitands to detect aromatic compounds in the vapor phase, for example in a benzene detection device. ${ }^{162}$ The quinoxaline cavitand possessed four decenyl groups which were bound by photochemical hydrosilylation, because the chance of retaining the molecular 
recognition properties were the highest for this mild functionalization method. Because of the bulkiness of the cavitand molecules, 1-octene was added to the reactant solution (mole fraction 0.8 ) in order to fully cover the $\mathrm{Si}-\mathrm{H}$ sites and thus prevent surface oxidation (Figure 2.15a). The sensing properties were tested using $\alpha, \alpha, \alpha$-trichlorotoluene (TCT), the chlorine moieties of which were used for detection by XPS. When the functionalized silicon surfaces were exposed to TCT vapor, XPS indeed confirmed complexation of about $80 \%$ of the host molecules, which did not occur for control samples with an inactive methylene-bridged cavitand. The molecular recognition was selective towards aromatic compounds, as tested by a mixture of TCT and bromo-containing linear and cyclic alkanes (Figure 2.15b). The host-guest interactions were reversible by heating up to $80^{\circ} \mathrm{C}$ for $15 \mathrm{~min}$ (Figure 2.15c), and the receptors could be reused thereafter. Similarly, Biavardi et al. covalently bound a tetraphosphonate cavitand onto $\mathrm{Si}-\mathrm{H}$, which is able to complex positively charged ions, such as ammonium and pyridinium salts, or neutral molecules, such as alcohols. ${ }^{163}$ The recognition of ammonium and pyridinium salts was confirmed using XPS and fluorescence spectroscopy. The complexation was reversible by using a base.
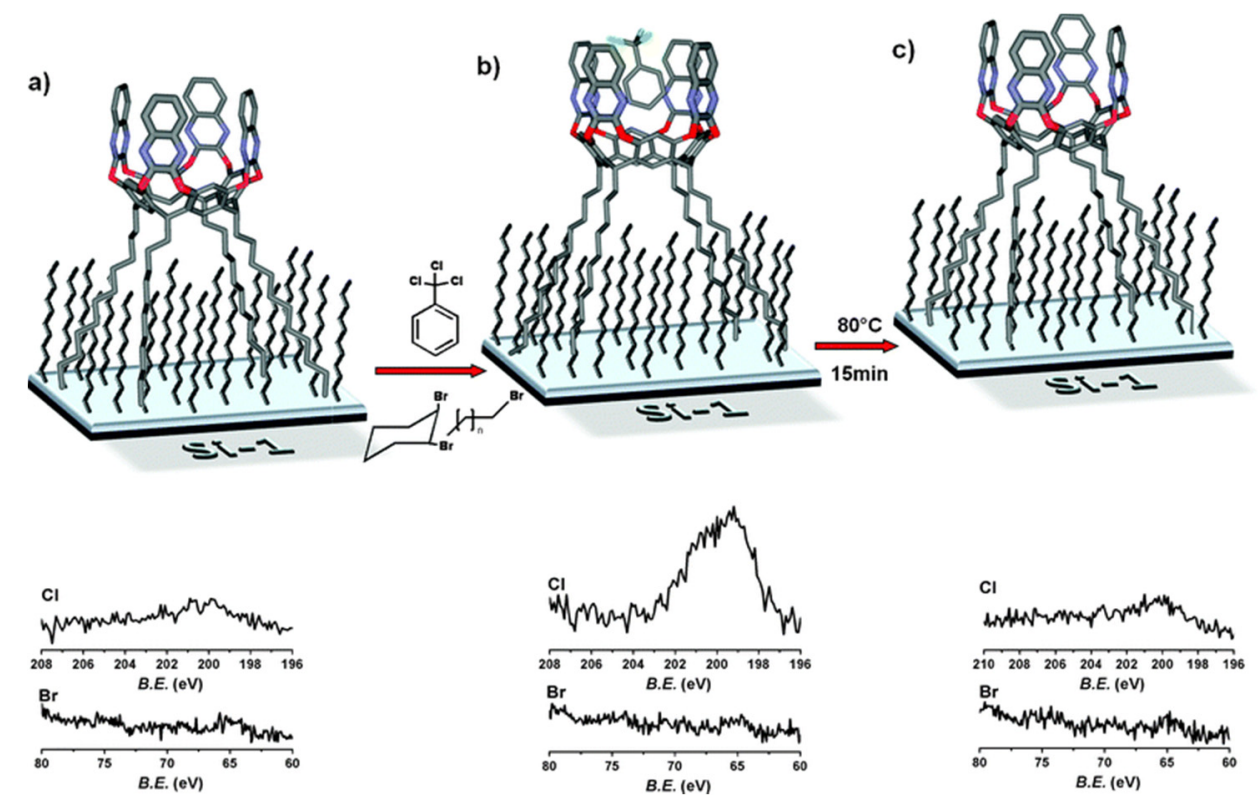

Figure 2.15. Molecular recognition steps as measured by XPS, including a) the quinoxaline cavitandfunctionalized silicon surface, b) competition between chlorine-tagged aromatic compounds and bromine-tagged linear and cyclic alkanes in the vapor phase, and c) decomplexation of the hostguest complex by heating. Reproduced with permission. ${ }^{162}$ Copyright 2010, The Royal Society of Chemistry. 
Another type of sensors is based on a change in optical properties, e.g., optical reflectivity changes after DNA hybridization ${ }^{164}$ or protein-protein interactions. ${ }^{165}$ Trojan et al. used the photoluminescence of porous silicon (see Section 2.6) to build a sensor device. ${ }^{166,167}$ They attached alkene-functionalized $\beta$-cyclodextrin molecules with different spacer lengths $\left(C_{4}, C_{7}, C_{11}\right)$ onto $\mathrm{H}$-terminated porous silicon. The photoluminescence signal of the porous silicon substrate without functionalization was quenched for several concentrations of analytes in the vapor phase (Figure 2.16a). When using a cyclodextrinfunctionalized substrate, however, the photoluminescence changed depending on the analyte concentration and the complexation strength (Figure 2.16b). The strongest recognition was observed for aromatic molecules, for which the photoluminescence was even enhanced for low analyte concentrations. These guests fit most optimally into the cyclodextrin host molecules and could thus not penetrate into the porous silicon matrix to quench the photoluminescence. The highest photoluminescence enhancement was observed for molecules with a $C_{7}$ spacer. For a shorter spacer length $\left(C_{4}\right)$, the packing density of the cyclodextrin molecules was lower, while for a longer spacer $\left(C_{11}\right)$ the electron transfer was less efficient. Although the stability of the monolayer-containing substrates was significantly higher, the limit of detection was increased by one or two orders of magnitude.
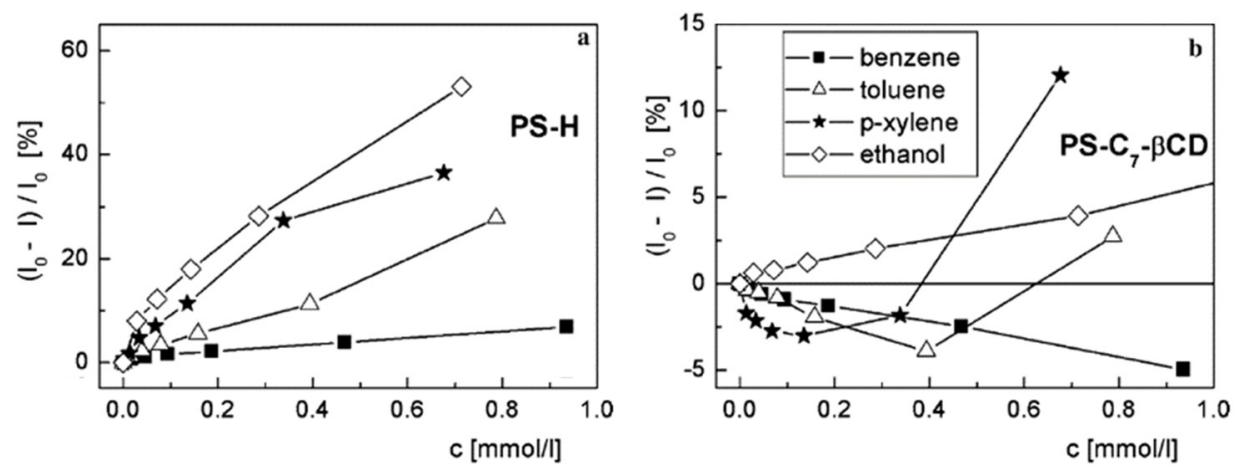

Figure 2.16. Relative photoluminescence intensities for a porous silicon substrate a) as prepared and b) with a $\beta$-cyclodextrin monolayer, with different analyte concentrations in the vapor phase. Reproduced with permission. ${ }^{166}$ Copyright 2007, Springer.

Zhu et al. investigated the use of porous silicon rugate filters, i.e., one-dimensional photonic crystals, as an optical sensor, which detects changes in the refractive index upon a reaction occurring inside the pores. ${ }^{168}$ The porous silicon substrate was functionalized with a monolayer of 1,8-nonadiyne by hydrosilylation. To be able to detect any changes, the pores were functionalized locally, i.e., only a part of freestanding alkyne moieties was reacted further by copper-catalyzed click-chemistry while selectively blocking the other areas with a titanium mask. The reflectivity spectra of the differently functionalized regions showed a redshift from 702.1 (alkyne moieties) to $720.5 \mathrm{~nm}$ (tetra(ethylene 
glycol) moieties). After mask removal, a second click chemistry reaction functionalized the initially masked 1,8-nonadiyne monolayer thus resulting in a chemical pattern. Functionalization with a tetra(ethylene glycol) chain with a hydroxyl moiety was successful and could potentially be used for biomolecule immobilization. This sensing platform has been used to detect cell adhesion ${ }^{169}$ and protease activity, which is a marker of immune response. ${ }^{170,171}$

Assad et al. built a sensing device based on a silicon nanowire FET that responds to analyte binding by changing conductivity. ${ }^{172}$ Any oxide layer lowered the response of the FET sensors, probably due to charge trapping. ${ }^{173}$ Therefore, Si-C bonds were formed on the silicon nanowires by binding alkyl groups by the chlorination/alkylation route. This device could detect polar and apolar compounds in the gas phase down to concentrations of $40 \mathrm{ppb}$. The apolar analyte molecules were adsorbed between the alkyl chains of the monolayer, which resulted in a conformational change of the monolayer and thus a change in conductivity of the silicon nanowire. For polar gas-phase molecules, the sensor response was higher and depended on the dipole moment of the analyte. When using an array of several monolayers, the device could discriminate between simulated vapors of "healthy" and "cancerous" breaths, which is a step towards an electronic nose for cancer diagnosis.

FETs are also commonly used for biosensing applications, mostly for DNA detection. Bunimovich et al. compared monolayers of amine-terminated silane and amineterminated alkyl molecules, onto which single-stranded DNA was electrostatically adsorbed. ${ }^{174}$ For this research, physiological conditions were mimicked by a $0.165 \mathrm{M}$ electrolyte solution, which promotes hybridization. On the other hand, the Debye screening length is lowered to about $1 \mathrm{~nm}$ in this medium, which requires the hybridization to occur close to the surface in order to be detected. Nonetheless, hybridization could be detected by a decrease of the resistance of the silicon nanowires. The devices based on oxide-free Si-C layers showed better FET characteristics and an increased sensitivity by one order of magnitude from $1 \mathrm{nM}$ to $<0.1 \mathrm{nM}$ (Figure 2.17). Another way to improve the sensitivity based on $\mathrm{Si}-\mathrm{C}^{175}$ or $\mathrm{Si}-\mathrm{N}^{176}$ bonds was explored by Masood et al. They created nanowire biosensors with selective functionalization of the nanowires only, in order to avoid the loss of analyte, and thus sensitivity, at the nondetected regions. Oxide-free functionalization was used to create amine-terminated monolayers at the silicon nanowire regions, onto which biotin groups were coupled, whereas the surrounding silicon oxide was not functionalized. Streptavidin-functionalized gold nanoparticles could then be selectively bound onto the silicon nanowire regions. Alternatively, selectively functionalized sensor areas can be achieved by immersion in a silane/alkene mixture, ${ }^{177}$ or by applying a potential on silicon wires to locally activate a hydroquinone monolayer. ${ }^{178}$ This oxidized benzoquinone form was then able to react 
with a biotin-functionalized cyclopentadiene in a Diels-Alder reaction, after which streptavidin molecules could only react onto the wired areas.
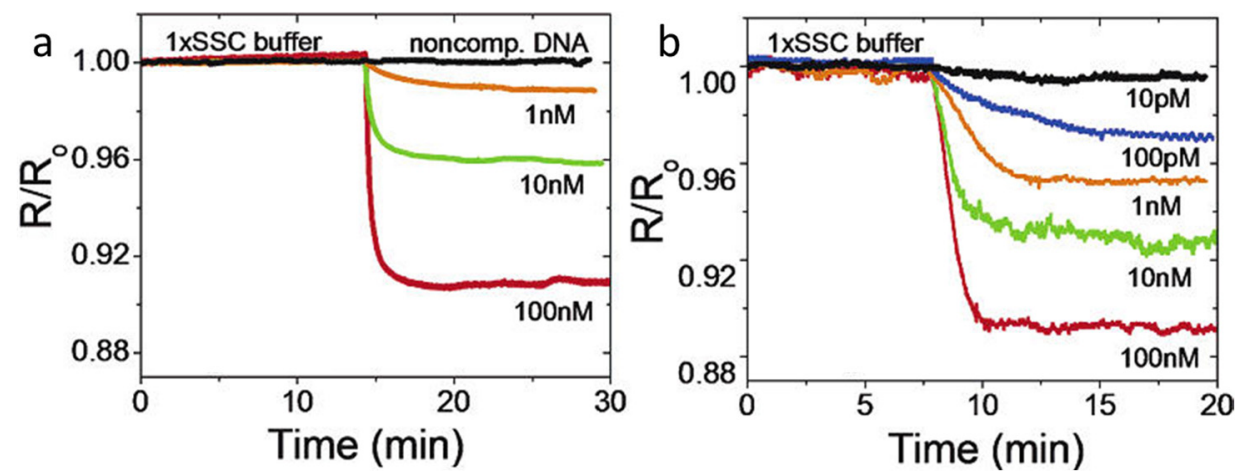

Figure 2.17. Real-time sensing of different concentrations of complementary DNA in $0.165 \mathrm{M}$ electrolyte by silicon nanowires, functionalized with a) an amine-terminated silane on $\mathrm{SiO}_{2}$ and b) amine-terminated alkyl molecules on $\mathrm{Si}-\mathrm{H}$. The black line in (a) represents exposure of the silicon nanowire sensor to $100 \mathrm{nM}$ noncomplementary DNA. Reproduced with permission. ${ }^{174}$ Copyright 2006, American Chemical Society.

Other electrical sensors are based on electrochemistry ${ }^{179}$ or impedance measurements, ${ }^{180-182}$ e.g., for the detection of DNA hybridization ${ }^{181,182}$ or antigenantibody interactions. ${ }^{179,180}$ As an example, Wei et al. reported the coupling of a singlestrand DNA sequence to a Si-C alkyl carboxylic acid monolayer for a DNA sensor based on impedance measurements, which avoids the use of an auxiliary redox couple or fluorescent labelling. ${ }^{182} \mathrm{~A}$ solution with $10 \mu \mathrm{M}$ complementary oligo DNA strands resulted in a real-time change in the capacitance, which was not observed for noncomplementary strands (Figure 2.18a). When starting from a substrate already saturated with complementary DNA, no signal change was observed as expected (Figure 2.18b). Dehybridization with a mixture of formamide and SDS at $60{ }^{\circ} \mathrm{C}$ for $10 \mathrm{~min}$ recovered the chip, which could then be reused (Figure 2.18c). This sensor was further optimized by the use of a hairpin strand as probe on the surface, since any hybridization would then open the hairpin structure and result in a higher sensitivity. ${ }^{183}$ 


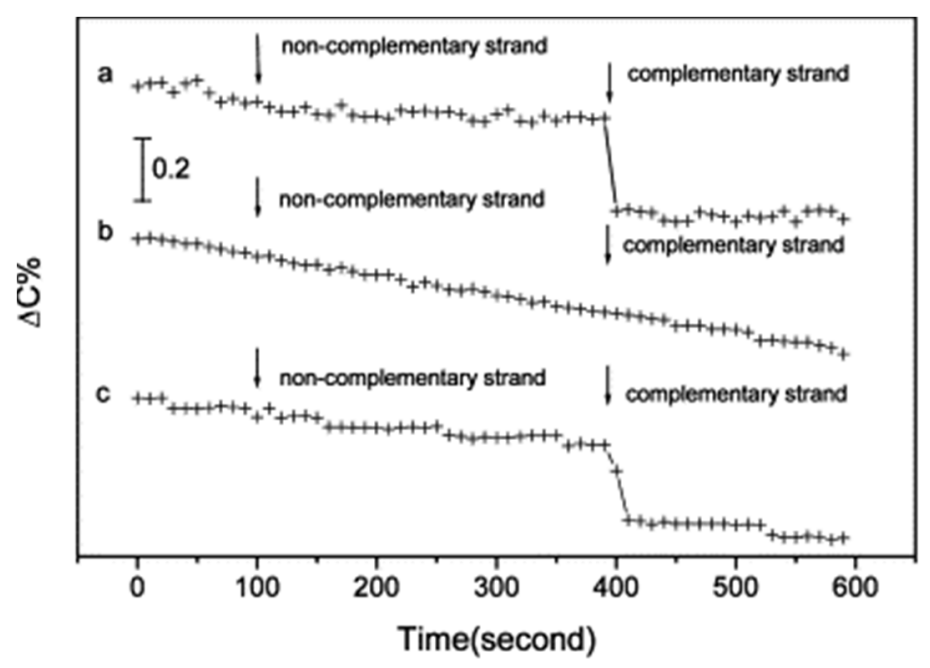

Figure 2.18. Real-time capacitance monitoring upon addition of (non)complementary DNA strands at an electrode modified with single-strand DNA a) as prepared, b) pre-hybridized with its complementary strand, and c) dehybridized for repeated use. Reproduced with permission. ${ }^{182}$ Copyright 2003, Elsevier.

Based on mass adsorption properties, Boiadjiev et al. built a cantilever-based sensor for $\mathrm{Cr}(\mathrm{VI})$ detection, which is relevant because the chromate anion $\left(\mathrm{CrO}_{4}{ }^{2-}\right)$ is toxic to animals and humans. ${ }^{184}$ They created a monolayer of a quaternary ammonium salt on $\mathrm{Si}-\mathrm{H}$ by photochemical hydrosilylation, which kept its affinity for chromate ions after functionalization. When an aqueous solution of $10^{-9}-10^{-4} \mathrm{M}$ chromate was passed over the microcantilever, deflection of the cantilever was detected upon adsorption of the ions onto the monolayer.

\subsection{Conclusions and outlook}

Oxide-free monolayer functionalization of silicon surfaces is a highly attractive method for the formation of stable, oxidation resistant monolayers. As a consequence, the amount of applications reported has rapidly expanded during the last two decades, as here subdivided into the fields of surface passivation, electronics, doping, optics, biomedical devices, and sensors. A wide range of molecules can be coupled to oxide-free molecules by the different routes available, including mild routes to avoid damage to labile molecules. Most of these routes lead to Si-C bonds, but Si-N, Si-O-C, and Si-S have been frequently reported as well. The monolayer headgroup can involve the desired functional moiety or a reactive group available for secondary functionalization. Any functional bulky molecules are mostly reacted on the surface by the latter multi-step procedure. These routes result in silicon surfaces that are either modulated at the siliconmolecule bond and/or obtain a new functionality due to the addition of a specific chemical group. Owing to this versatility, the number of applications is expected to keep 
increasing, especially in the area of silicon micro/nanostructures where molecular monolayers go beyond the limits of conventional fabrication and functionalization techniques.

\subsection{References}

1. J.J. Gooding and S. Ciampi, Chem. Soc. Rev., 2011, 40, 2704-2718.

2. A. Ulman, Chem. Rev., 1996, 96, 1533-1554.

3. M.R. Linford and C.E.D. Chidsey, J. Am. Chem. Soc., 1993, 115, 12631-12632.

4. N.S. Bhairamadgi, S.P. Pujari, F.G. Trovela, A. Debrassi, A.A. Khamis, J.M. Alonso, A.A. Al Zahrani, T. Wennekes, H.A. Al-Turaif, C. van Rijn, Y.A. Alhamed and H. Zuilhof, Langmuir, 2014, 30, 5829-5839.

5. N. Shirahata, A. Hozumi and T. Yonezawa, Chem. Rec., 2005, 5, 145-159.

6. S. Ciampi, J.B. Harper and J.J. Gooding, Chem. Soc. Rev., 2010, 39, 2158-2183.

7. J.M. Buriak, Chem. Commun., 1999, 1051-1060.

8. J.M. Buriak, Chem. Rev., 2002, 102, 1271-1308.

9. D.D.M. Wayner and R.A. Wolkow, J. Chem. Soc., Perkin Trans. 2, 2002, 23-34.

10. G. Collins and J.D. Holmes, J. Mater. Chem., 2011, 21, 11052-11069.

11. R. Boukherroub, Curr. Opin. Solid State Mater. Sci., 2005, 9, 66-72.

12. P. Thissen, O. Seitz and Y.J. Chabal, Prog. Surf. Sci., 2012, 87, 272-290.

13. K.A. Perrine and A.V. Teplyakov, Chem. Soc. Rev., 2010, 39, 3256-3274.

14. A.B. Sieval, R. Linke, H. Zuilhof and E.J.R. Sudholter, Adv. Mater., 2000, 12, 1457-1460.

15. A.B. Sieval, A.L. Demirel, J.W.M. Nissink, M.R. Linford, J.H. van der Maas, W.H. de Jeu, H. Zuilhof and E.J.R. Sudholter, Langmuir, 1998, 14, 1759-1768.

16. A.B. Sieval, R. Opitz, H.P.A. Maas, M.G. Schoeman, G. Meijer, F.J. Vergeldt, H. Zuilhof and E.J.R. Sudholter, Langmuir, 2000, 16, 10359-10368.

17. Q.Y. Sun, L.C.P.M. de Smet, B. van Lagen, M. Giesbers, P.C. Thune, J. van Engelenburg, F.A. de Wolf, H. Zuilhof and E.J.R. Sudholter, J. Am. Chem. Soc., 2005, 127, 2514-2523.

18. R.L. Cicero, M.R. Linford and C.E.D. Chidsey, Langmuir, 2000, 16, 5688-5695.

19. S. Ciampi, E. Luais, M. James, M.H. Choudhury, N.A. Darwish and J.J. Gooding, Phys. Chem. Chem. Phys., 2014, 16, 8003-8011.

20. L. Scheres, A. Arafat and H. Zuilhof, Langmuir, 2007, 23, 8343-8346.

21. A.B. Sieval, V. Vleeming, H. Zuilhof and E.J.R. Sudholter, Langmuir, 1999, 15, 8288-8291.

22. E.G. Robins, M.P. Stewart and J.M. Buriak, Chem. Commun., 1999, 2479-2480.

23. D. Belanger and J. Pinson, Chem. Soc. Rev., 2011, 40, 3995-4048.

24. D.K. Aswal, S.P. Koiry, B. Jousselme, S.K. Gupta, S. Palacin and J.V. Yakhmi, Phys. E, 2009, 41, 325-344.

25. G. Cleland, B.R. Horrocks and A. Houlton, J. Chem. Soc., Faraday Trans., 1995, 91, 4001-4003.

26. M.Y. Bashouti, K. Sardashti, S.W. Schmitt, M. Pietsch, J. Ristein, H. Haick and S.H. Christiansen, Prog. Surf. Sci., 2013, 88, 39-60.

27. K.T. Wong and N.S. Lewis, Acc. Chem. Res., 2014, 47, 3037-3044.

28. R. Boukherroub, S. Morin, F. Bensebaa and D.D.M. Wayner, Langmuir, 1999, 15, 3831-3835.

29. T.P. Chopra, R.C. Longo, K. Cho and Y.J. Chabal, Surf. Sci., 2016, 650, 285-294.

30. W.F. Bergerson, J.A. Mulder, R.P. Hsung and X.Y. Zhu, J. Am. Chem. Soc., 1999, 121, 454-455. 
31. F.Y. Tian and A.V. Teplyakov, Langmuir, 2013, 29, 13-28.

32. X.Y. Zhu, V. Boiadjiev, J.A. Mulder, R.P. Hsung and R.C. Major, Langmuir, 2000, 16, 6766-6772.

33. M.P. Stewart, F. Maya, D.V. Kosynkin, S.M. Dirk, J.J. Stapleton, C.L. McGuiness, D.L. Allara and J.M. Tour, J. Am. Chem. Soc., 2004, 126, 370-378.

34. P. Allongue, C.H. de Villeneuve, J. Pinson, F. Ozanam, J.N. Chazalviel and X. Wallart, Electrochim. Acta, 1998, 43, 2791-2798.

35. R.C. Longo, K. Cho, W.G. Schmidt, Y.J. Chabal and P. Thissen, Adv. Funct. Mater., 2013, 23, 3471-3477.

36. L.H. Yu, N. Gergel-Hackett, C.D. Zangmeister, C.A. Hacker, C.A. Richter and J.G. Kushmerick, J. Phys.: Condens. Matter, 2008, 20, 374114.

37. J.M. Buriak and M.D.H. Sikder, J. Am. Chem. Soc., 2015, 137, 9730-9738.

38. M.J. Hu, F.L. Liu and J.M. Buriak, ACS Appl. Mater. Interfaces, 2016, 8, 11091-11099.

39. G. Shambat, A. Deberardinis, B. Chen, P. Reinke, B.J. Venton, L. Pu, J. Tour and J. Bean, Appl. Surf. Sci., 2009, 255, 8533-8538.

40. M.Y. Bashouti, T. Stelzner, A. Berger, S. Christiansen and H. Haick, J. Phys. Chem. C, 2008, 112, 19168-19172.

41. M.Y. Bashouti, T. Stelzner, S. Christiansen and H. Haick, J. Phys. Chem. C, 2009, 113, 1482314828.

42. O. Assad, S.R. Puniredd, T. Stelzner, S. Christiansen and H. Haick, J. Am. Chem. Soc., 2008, 130, 17670-17671.

43. S. Ciampi, P.K. Eggers, G. Le Saux, M. James, J.B. Harper and J.J. Gooding, Langmuir, 2009, 25, 2530-2539.

44. Q. Nguyen Minh, S.P. Pujari, B. Wang, Z. Wang, H. Haick, H. Zuilhof and C.J.M. van Rijn, Appl. Surf. Sci., 2016, 387, 1202-1210.

45. K.E. Plass, X.L. Liu, B.S. Brunschwig and N.S. Lewis, Chem. Mater., 2008, 20, 2228-2233.

46. A.B. Sieval, C.L. Huisman, A. Schonecker, F.M. Schuurmans, A.S.H. van der Heide, A. Goossens, W.C. Sinke, H. Zuilhof and E.J.R. Sudholter, J. Phys. Chem. B, 2003, 107, 6846-6852.

47. E.J. Nemanick, P.T. Hurley, L.J. Webb, D.W. Knapp, D.J. Michalak, B.S. Brunschwig and N.S. Lewis, J. Phys. Chem. B, 2006, 110, 14770-14778.

48. E.J. Nemanick, P.T. Hurley, B.S. Brunschwig and N.S. Lewis, J. Phys. Chem. B, 2006, 110, 1480014808.

49. N. Alderman, L. Danos, M.C. Grossel and T. Markvart, RSC Adv., 2013, 3, 20125-20131.

50. C.J. Barrelet, D.B. Robinson, J. Cheng, T.P. Hunt, C.F. Quate and C.E.D. Chidsey, Langmuir, 2001, 17, 3460-3465.

51. R. Boukherroub, S. Morin, D.D.M. Wayner, F. Bensebaa, G.I. Sproule, J.M. Baribeau and D.J. Lockwood, Chem. Mater., 2001, 13, 2002-2011.

52. C.A. Hacker, K.A. Anderson, L.J. Richter and C.A. Richter, Langmuir, 2005, 21, 882-889.

53. O. Yaffe, T. Ely, R. Har-Lavan, D.A. Egger, S. Johnston, H. Cohen, L. Kronik, A. Vilan and D. Cahen, J. Phys. Chem. C, 2013, 117, 22351-22361.

54. L.J. Webb and N.S. Lewis, J. Phys. Chem. B, 2003, 107, 5404-5412.

55. A.G. Aberle, Prog. Photovoltaics, 2000, 8, 473-487.

56. M.Y. Bashouti, J. Ristein, H. Haick and S. Christiansen, Hybrid Mater., 2014, 1, 2-14.

57. M.V. Fernandez-Serra, C. Adessi and X. Blase, Nano Lett., 2006, 6, 2674-2678.

58. F. Zhang, D. Liu, Y. Zhang, H. Wei, T. Song and B. Sun, ACS Appl. Mater. Interfaces, 2013, 5, 4678-4684. 
59. G.F. Moore and I.D. Sharp, J. Phys. Chem. Lett., 2013, 4, 568-572.

60. X.J. Shen, B.Q. Sun, F. Yan, J. Zhao, F.T. Zhang, S.D. Wang, X.L. Zhu and S. Lee, ACS Nano, 2010, 4, 5869-5876.

61. B. Fabre, Chem. Rev., 2016, 116, 4808-4849.

62. K. Huang, F. Duclairoir, T. Pro, J. Buckley, G. Marchand, E. Martinez, J.-C. Marchon, B. De Salvo, G. Delapierre and F. Vinet, ChemPhysChem, 2009, 10, 963-971.

63. A.G. Marrani, F. Cattaruzza, F. Decker, R. Zanoni, M. Cossi and M.F. Iozzi, J. Nanosci. Nanotechnol., 2010, 10, 2901-2907.

64. Y. Yang, S. Ciampi, M.H. Choudhury and J.J. Gooding, J. Phys. Chem. C, 2016, 120, 2874-2882.

65. H. Liu, F. Duclairoir, B. Fleury, L. Dubois, Y. Chenavier and J.C. Marchon, Dalton Trans., 2009, 3793-3799.

66. G. Yzambart, B. Fabre, F. Camerel, T. Roisnel and D. Lorcy, J. Phys. Chem. C, 2012, 116, 1209312102.

67. G. Yzambart, B. Fabre and D. Lorcy, Langmuir, 2012, 28, 3453-3459.

68. W.J. Feng and B. Miller, Langmuir, 1999, 15, 3152-3156.

69. R. Har-Lavan, O. Yaffe, P. Joshi, R. Kazaz, H. Cohen and D. Cahen, AIP Adv., 2012, 2, 012164.

70. E.J. Faber, L.C.P.M. de Smet, W. Olthuis, H. Zuilhof, E.J.R. Sudholter, P. Bergveld and A. van den Berg, ChemPhysChem, 2005, 6, 2153-2166.

71. A. Vilan, O. Yaffe, A. Biller, A. Salomon, A. Kahn and D. Cahen, Adv. Mater., 2010, 22, 140-159.

72. F. Thieblemont, O. Seitz, A. Vilan, H. Cohen, E. Salomon, A. Kahn and D. Cahen, Adv. Mater., 2008, 20, 3931-3936.

73. Y.J. Liu and H.Z. Yu, J. Phys. Chem. B, 2003, 107, 7803-7811.

74. Y.S. Cohen, A. Vilan, I. Ron and D. Cahen, J. Phys. Chem. C, 2009, 113, 6174-6181.

75. T. Böcking, A. Salomon, D. Cahen and J.J. Gooding, Langmuir, 2007, 23, 3236-3241.

76. H. Asanuma, E.M. Bishop and H.Z. Yu, Electrochim. Acta, 2007, 52, 2913-2919.

77. S.P. Pujari, E. van Andel, O. Yaffe, D. Cahen, T. Weidner, C.J.M. van Rijn and H. Zuilhof, Langmuir, 2013, 29, 570-580.

78. O. Seitz, T. Böcking, A. Salomon, J.J. Gooding and D. Cahen, Langmuir, 2006, 22, 6915-6922.

79. Z.M. Liu, A.A. Yasseri, J.S. Lindsey and D.F. Bocian, Science, 2003, 302, 1543-1545.

80. B. Fabre, Y. Li, L. Scheres, S.P. Pujari and H. Zuilhof, Angew. Chem., Int. Ed., 2013, 52, 1202412027.

81. B. Fabre, S.P. Pujari, L. Scheres and H. Zuilhof, Langmuir, 2014, 30, 7235-7243.

82. Q.L. Li, G. Mathur, S. Gowda, S. Surthi, Q. Zhao, L.H. Yu, J.S. Lindsey, D.F. Bocian and V. Misra, Adv. Mater., 2004, 16, 133-137.

83. L. Ye, M.P. de Jong, T. Kudernac, W.G. van der Wiel and J. Huskens, Mater. Sci. Semicond. Process., 2017, 62, 128-134.

84. J. O'Connell, S. Biswas, R. Duffy and J.D. Holmes, Nanotechnology, 2016, 27, 342002.

85. J.C. Ho, R. Yerushalmi, Z.A. Jacobson, Z. Fan, R.L. Alley and A. Javey, Nat. Mater., 2008, 7, 6267.

86. J.C. Ho, R. Yerushalmi, G. Smith, P. Majhi, J. Bennett, J. Halim, V.N. Faifer and A. Javey, Nano Lett., 2009, 9, 725-730.

87. L. Ye, S.P. Pujari, H. Zuilhof, T. Kudernac, M.P. de Jong, W.G. van der Wiel and J. Huskens, ACS Appl. Mater. Interfaces, 2015, 7, 3231-3236.

88. L. Ye, A. Gonzalez-Campo, R. Nunez, M.P. de Jong, T. Kudernac, W.G. van der Wiel and J. Huskens, ACS Appl. Mater. Interfaces, 2015, 7, 27357-27361. 
89. J. O'Connell, G.A. Verni, A. Gangnaik, M. Shayesteh, B. Long, Y.M. Georgiev, N. Petkov, G.P. McGlacken, M.A. Morris, R. Duffy and J.D. Holmes, ACS Appl. Mater. Interfaces, 2015, 7, 1551415521.

90. R.C. Longo, E.C. Mattson, A. Vega, W. Cabrera, K. Cho, Y.J. Chabal and P. Thissen, Chem. Mater., 2016, 28, 1975-1979.

91. J. O'Connell, G. Collins, G.P. McGlacken, R. Duffy and J.D. Holmes, ACS Appl. Mater. Interfaces, 2016, 8, 4101-4108.

92. B. Guan, H. Siampour, Z. Fan, S. Wang, X.Y. Kong, A. Mesli, J. Zhang and Y.P. Dan, Sci. Rep., 2015, 5, 12641.

93. I. Ok, K.W. Ang, C. Hobbs, R.H. Baek, C.Y. Kang, J. Snow, P. Nunan, S. Nadahara, P.D. Kirsch and R. Jammy, in 12th International Workshop on Junction Technology, Shanghai, 2012, 29-34.

94. M.P.V. Kumar, C.Y. Hu, K.H. Kao, Y.J. Lee and T.S. Chao, IEEE Trans. Electron Devices, 2015, 3541-3546.

95. W.P. Voorthuijzen, M.D. Yilmaz, W.J.M. Naber, J. Huskens and W.G. van der Wiel, Adv. Mater., 2011, 23, 1346-1350.

96. O. Hazut, A. Agarwala, I. Amit, T. Subramani, S. Zaidiner, Y. Rosenwaks and R. Yerushalmi, ACS Nano, 2012, 6, 10311-10318.

97. O. Hazut, B.C. Huang, A. Pantzer, I. Amit, Y. Rosenwaks, A. Kohn, C.S. Chang, Y.P. Chiu and R. Yerushalmi, ACS Nano, 2014, 8, 8357-8362.

98. R.A. Puglisi, C. Garozzo, C. Bongiorno, S. Di Franco, M. Italia, G. Mannino, S. Scalese and A. La Magna, Sol. Energy Mater. Sol. Cells, 2015, 132, 118-122.

99. C. Garozzo, F. Giannazzo, M. Italia, A. La Magna, V. Privitera and R.A. Puglisi, Mater. Sci. Eng., B, 2013, 178, 686-690.

100. C. Garozzo, C. Bongiorno, S. Di Franco, M. Italia, A. La Magna, S. Scalese, P.M. Sberna and R.A. Puglisi, Phys. Status Solidi A, 2013, 210, 1564-1570.

101. W. Chen, D.C. Qi, X.Y. Gao and A.T.S. Wee, Prog. Surf. Sci., 2009, 84, 279-321.

102. J. Ristein, Science, 2006, 313, 1057-1058.

103. T. He, H.J. Ding, N. Peor, M. Lu, D.A. Corley, B. Chen, Y. Ofir, Y.L. Gao, S. Yitzchaik and J.M. Tour, J. Am. Chem. Soc., 2008, 130, 1699-1710.

104. X.Y. Zhang, Y.Q. Wen, Y.F. Li, G. Li, S.X. Du, H.M. Guo, L.M. Yang, L. Jiang, H.J. Gao and Y. Song, J. Phys. Chem. C, 2008, 112, 8288-8293.

105. A. Miranda-Duran, X. Cartoixa, M.C. Irisson and R. Rurali, Nano Lett., 2010, 10, 3590-3595.

106. R. Haight, L. Sekaric, A. Afzali and D. Newns, Nano Lett., 2009, 9, 3165-3170.

107. M.Y. Bashouti, R.T. Tung and H. Haick, Small, 2009, 5, 2761-2769.

108. C. Suspene, R. Barattin, C. Celle, A. Carella and J.P. Simonato, J. Phys. Chem. C, 2010, 114, 39243931.

109. T. He, J.L. He, M. Lu, B. Chen, H. Pang, W.F. Reus, W.M. Nolte, D.P. Nackashi, P.D. Franzon and J.M. Tour, J. Am. Chem. Soc., 2006, 128, 14537-14541.

110. R. Har-Lavan, I. Ron, F. Thieblemont and D. Cahen, Appl. Phys. Lett., 2009, 94, 043308.

111. B. Hamilton, Semicond. Sci. Technol., 1995, 10, 1187-1207.

112. G. Belomoin, J. Therrien, A. Smith, S. Rao, R. Twesten, S. Chaieb, M.H. Nayfeh, L. Wagner and L. Mitas, Appl. Phys. Lett., 2002, 80, 841-843.

113. X.G. Li, Y.Q. He and M.T. Swihart, Langmuir, 2004, 20, 4720-4727.

114. M. Miyano, T. Nakanishi, S. Wada, Y. Kitagawa, A. Kawashima, K. Fushimi, Y. Morisaki, Y. Chujo and Y. Hasegawa, J. Photopolym. Sci. Technol., 2015, 28, 255-260. 
115. R. Boukherroub, S. Morin, D.D.M. Wayner and D.J. Lockwood, Phys. Status Solidi A, 2000, 182, 117-121.

116. J.H. Song and M.J. Sailor, Inorg. Chem., 1999, 38, 1498-1503.

117. J.H. Song and M.J. Sailor, J. Am. Chem. Soc., 1998, 120, 2376-2381.

118. B. Sweryda-Krawiec, T. Cassagneau and J.H. Fendler, J. Phys. Chem. B, 1999, 103, 9524-9529.

119. H.C. Choi and J.M. Buriak, Chem. Mater., 2000, 12, 2151-2156.

120. R. Boukherroub, D.D.M. Wayner, G.I. Sproule, D.J. Lockwood and L.T. Canham, Nano Lett., 2001, 1, 713-717.

121. M.P. Stewart and J.M. Buriak, Angew. Chem., Int. Ed., 1998, 37, 3257-3260.

122. M.P. Stewart and J.M. Buriak, J. Am. Chem. Soc., 2001, 123, 7821-7830.

123. J. Zou, R.K. Baldwin, K.A. Pettigrew and S.M. Kauzlarich, Nano Lett., 2004, 4, 1181-1186.

124. M. Miyano, S. Endo, H. Takenouchi, S. Nakamura, Y. Iwabuti, O. Shiino, T. Nakanishi and Y. Hasegawa, J. Phys. Chem. C, 2014, 118, 19778-19784.

125. M. Miyano, Y. Kitagawa, S. Wada, A. Kawashima, A. Nakajima, T. Nakanishi, J. Ishioka, T. Shibayama, S. Watanabe and Y. Hasegawa, Photochem. Photobiol. Sci., 2016, 15, 99-104.

126. M. Rosso-Vasic, E. Spruijt, B. van Lagen, L. De Cola and H. Zuilhof, Small, 2008, 4, 1835-1841.

127. S. Di Bella, G.G. Condorelli, A. Motta, A. Ustione and A. Cricenti, Langmuir, 2006, 22, 79527955.

128. G.G. Condorelli, C. Tudisco, A. Motta, A. Di Mauro, F. Lupo, A. Gulino and I.L. Fragala, Eur. J. Inorg. Chem., 2010, 4121-4129.

129. M.J. Sweetman, F.J. Harding, S.D. Graney and N.H. Voelcker, Appl. Surf. Sci., 2011, 257, 67686774.

130. L.T. Canham, C.L. Reeves, J.P. Newey, M.R. Houlton, T.I. Cox, J.M. Buriak and M.P. Stewart, Adv. Mater., 1999, 11, 1505-1507.

131. T. Böcking, K.A. Kilian, K. Gaus and J.J. Gooding, Adv. Funct. Mater., 2008, 18, 3827-3833.

132. L.C.P.M. de Smet, G.A. Stork, G.H.F. Hurenkamp, Q.Y. Sun, H. Topal, P.J.E. Vronen, A.B. Sieval, A. Wright, G.M. Visser, H. Zuilhof and E.J.R. Sudholter, J. Am. Chem. Soc., 2003, 125, 1391613917.

133. L.C.P.M. de Smet, A.V. Pukin, Q.-Y. Sun, B.J. Eves, G.P. Lopinski, G.M. Visser, H. Zuilhof and E.J.R. Sudhölter, Appl. Surf. Sci., 2005, 252, 24-30.

134. N. Shirahata, T. Yonezawa, Y. Miura, K. Kobayashi and K. Koumoto, Langmuir, 2003, 19, 91079109.

135. M. Yang, R.L.M. Teeuwen, M. Giesbers, J. Baggerman, A. Arafat, F.A. de Wolf, J.C.M. van Hest and H. Zuilhof, Langmuir, 2008, 24, 7931-7938.

136. G.T. Qin, C. Santos, W. Zhang, Y. Li, A. Kumar, U.J. Erasquin, K. Liu, P. Muradov, B.W. Trautner and C.Z. Cai, J. Am. Chem. Soc., 2010, 132, 16432-16441.

137. M.A. Caipa Campos, J.M.J. Paulusse and H. Zuilhof, Chem. Commun., 2010, 46, 5512-5514.

138. L. Ruizendaal, S.P. Pujari, V. Gevaerts, J.M.J. Paulusse and H. Zuilhof, Chem. - Asian J., 2011, 6, 2776-2786.

139. T. Strother, W. Cai, X.S. Zhao, R.J. Hamers and L.M. Smith, J. Am. Chem. Soc., 2000, 122, 12051209.

140. A.R. Pike, L.H. Lie, R.A. Eagling, L.C. Ryder, S.N. Patole, B.A. Connolly, B.R. Horrocks and A. Houlton, Angew. Chem., Int. Ed., 2002, 41, 615-617.

141. F. Cattaruzza, A. Cricenti, A. Flamini, M. Girasole, G. Longo, T. Prosperi, G. Andreano, L. Cellai and E. Chirivino, Nucleic Acids Res., 2006, 34, e32. 
142. L. Scheres, J. ter Maat, M. Giesbers and H. Zuilhof, Small, 2010, 6, 642-650.

143. B. Guan, S. Ciampi, G. Le Saux, K. Gaus, P.J. Reece and J.J. Gooding, Langmuir, 2011, 27, 328334.

144. M. Rosso, M. Giesbers, K. Schroen and H. Zuilhof, Langmuir, 2010, 26, 866-872.

145. B.S. Flavel, A.J. Gross, D.J. Garrett, V. Nock and A.J. Downard, ACS Appl. Mater. Interfaces, 2010, 2, 1184-1190.

146. J. Choi, N.S. Wang and V. Reipa, Bioconjugate Chem., 2008, 19, 680-685.

147. W. Liao, F. Wei, M.X. Qian and X.S. Zhao, Sens. Actuators, B, 2004, 101, 361-367.

148. Z.R. Scheibal, W. Xu, J.F. Audiffred, J.E. Henry and J.C. Flake, Electrochem. Solid-State Lett., 2008, 11, K81-K84.

149. D.J. Guo, S.J. Xiao, B. Xia, Shuai-Wei, J. Pei, Y. Pan, X.Z. You, Z.Z. Gu and Z.H. Lu, J. Phys. Chem. B, 2005, 109, 20620-20628.

150. L. Wang, V. Reipa and J. Blasic, Bioconjugate Chem., 2004, 15, 409-412.

151. B.R. Hart, S.E. Letant, S.R. Kane, M.Z. Hadi, S.J. Shields and J.G. Reynolds, Chem. Commun., 2003, 322-323.

152. B.S. Flavel, M. Jasieniak, L. Velleman, S. Ciampi, E. Luais, J.R. Peterson, H.J. Griesser, J.G. Shapter and J.J. Gooding, Langmuir, 2013, 29, 8355-8362.

153. K.A. Kilian, T. Böcking, S. Ilyas, K. Gaus, W. Jessup, M. Gal and J.J. Gooding, Adv. Funct. Mater., 2007, 17, 2884-2890.

154. C.M. Yam, J.M. Lopez-Romero, J.H. Gu and C.Z. Cai, Chem. Commun., 2004, 2510-2511.

155. C.C.A. Ng, S. Ciampi, J.B. Harper and J.J. Gooding, Surf. Sci., 2010, 604, 1388-1394.

156. E.C. Wu, J.S. Andrew, L.Y. Cheng, W.R. Freeman, L. Pearson and M.J. Sailor, Biomaterials, 2011, 32, 1957-1966.

157. K.L. Jarvis, T.J. Barnes and C.A. Prestidge, Adv. Colloid Interface Sci., 2012, 175, 25-38.

158. E.J. Anglin, M.P. Schwartz, V.P. Ng, L.A. Perelman and M.J. Sailor, Langmuir, 2004, 20, $11264-$ 11269.

159. M. Kovalainen, J. Monkare, E. Makila, J. Salonen, V.P. Lehto, K.H. Herzig and K. Jarvinen, Pharm. Res., 2012, 29, 837-846.

160. J.S. Park, J.M. Kinsella, D.D. Jandial, S.B. Howell and M.J. Sailor, Small, 2011, 7, 2061-2069.

161. E.C. Wu, J.H. Park, J. Park, E. Segal, F. Cunin and M.J. Sailor, ACS Nano, 2008, 2, 2401-2409.

162. G.G. Condorelli, A. Motta, M. Favazza, E. Gurrieri, P. Betti and E. Dalcanale, Chem. Commun., 2010, 46, 288-290.

163. E. Biavardi, M. Favazza, A. Motta, I.L. Fragala, C. Massera, L. Prodi, M. Montalti, M. Melegari, G.G. Condorelli and E. Dalcanale, J. Am. Chem. Soc., 2009, 131, 7447-7455.

164. L. De Stefano, L. Rotiroti, I. Rea, L. Moretti, G. Di Francia, E. Massera, A. Lamberti, P. Arcari, C. Sanges and I. Rendina, J. Opt. A: Pure Appl. Opt., 2006, 8, S540-S544.

165. L. De Stefano, M. Rossi, M. Staiano, G. Mamone, A. Parracino, L. Rotiroti, I. Rendina, M. Rossi and S. D'Auria, J. Proteome Res., 2006, 5, 1241-1245.

166. T. Trojan, J. Jindrich, V. Vrkoslav, I. Jelinek and J. Dian, J. Inclusion Phenom. Macrocyclic Chem., 2007, 57, 343-348.

167. V. Vrkoslav, I. Jelinek, T. Trojan, J. Jindrich and J. Dian, Phys. E, 2007, 38, 200-204.

168. Y. Zhu, B. Gupta, B. Guan, S. Ciampi, P.J. Reece and J.J. Gooding, ACS Appl. Mater. Interfaces, 2013, 5, 6514-6521.

169. K.A. Kilian, T. Böcking, K. Gaus and J.J. Gooding, Angew. Chem., Int. Ed., 2008, 47, 2697-2699.

170. M.M. Orosco, C. Pacholski, G.M. Miskelly and M.J. Sailor, Adv. Mater., 2006, 18, 1393-1396. 
171. K.A. Kilian, T. Boecking, K. Gaus, M. Gal and J.J. Gooding, ACS Nano, 2007, 1, 355-361.

172. O. Assad and H. Haick, IEEE Int. Symp. Ind. Electron., 2008, 2040-2044.

173. H. Haick, P.T. Hurley, A.I. Hochbaum, P. Yang and N.S. Lewis, J. Am. Chem. Soc., 2006, 128, 8990-8991.

174. Y.L. Bunimovich, Y.S. Shin, W.S. Yeo, M. Amori, G. Kwong and J.R. Heath, J. Am. Chem. Soc., 2006, 128, 16323-16331.

175. M.N. Masood, S. Chen, E.T. Carlen and A. van den Berg, ACS Appl. Mater. Interfaces, 2010, 2, 3422-3428.

176. M.N. Masood, E.T. Carlen and A. van den Berg, Appl. Surf. Sci., 2015, 337, 105-110.

177. O. Seitz, P.G. Fernandes, G.A. Mahmud, H.C. Wen, H.J. Stiegler, R.A. Chapman, E.M. Vogel and Y.J. Chabal, Langmuir, 2011, 27, 7337-7340.

178. Y.L. Bunimovich, G.L. Ge, K.C. Beverly, R.S. Ries, L. Hood and J.R. Heath, Langmuir, 2004, 20, 10630-10638.

179. G.Z. Liu, M.N. Paddon-Row and J.J. Gooding, Chem. Commun., 2008, 3870-3872.

180. W.S. Yang, J.E. Butler, J.N. Russell and R.J. Hamers, Analyst, 2007, 132, 296-306.

181. W. Cai, J.R. Peck, D.W. van der Weide and R.J. Hamers, Biosens. Bioelectron., 2004, 19, 10131019.

182. F. Wei, B. Sun, Y. Guo and X.S. Zhao, Biosens. Bioelectron., 2003, 18, 1157-1163.

183. F. Wei, B. Sun, W. Liao, J.H. Ouyang and X.S. Zhao, Biosens. Bioelectron., 2003, 18, 1149-1155.

184. V.I. Boiadjiev, G.M. Brown, L.A. Pinnaduwage, G. Goretzki, P.V. Bonnesen and T. Thundat, Langmuir, 2005, 21, 1139-1142. 


\section{Chapter 3}

\section{Molecular monolayers for electrical passivation and functionalization of silicon-based solar energy devices}

Silicon-based solar fuel devices require passivation for optimal performance yet at the same time need functionalization with (photo)catalysts for efficient solar fuel production. Here, we use molecular monolayers to enable electrical passivation and simultaneous functionalization of silicon-based solar cells. Organic monolayers were coupled to silicon surfaces by hydrosilylation in order to avoid an insulating silicon oxide layer at the surface. Monolayers of 1-tetradecyne were shown to passivate silicon micropillar-based solar cells with radial junctions, by which the efficiency increased from $8.7 \%$ to $9.9 \%$ for $\mathrm{n}^{+} / \mathrm{p}$ junctions and from $7.8 \%$ to $8.8 \%$ for $\mathrm{p}^{+} / \mathrm{n}$ junctions. This electrical passivation of the surface, most likely by removal of dangling bonds, is reflected in a higher shunt resistance in the $J-V$ measurements. Monolayers of 1,8-nonadiyne were still reactive for click chemistry with a model catalyst, thus enabling simultaneous passivation and future catalyst coupling.

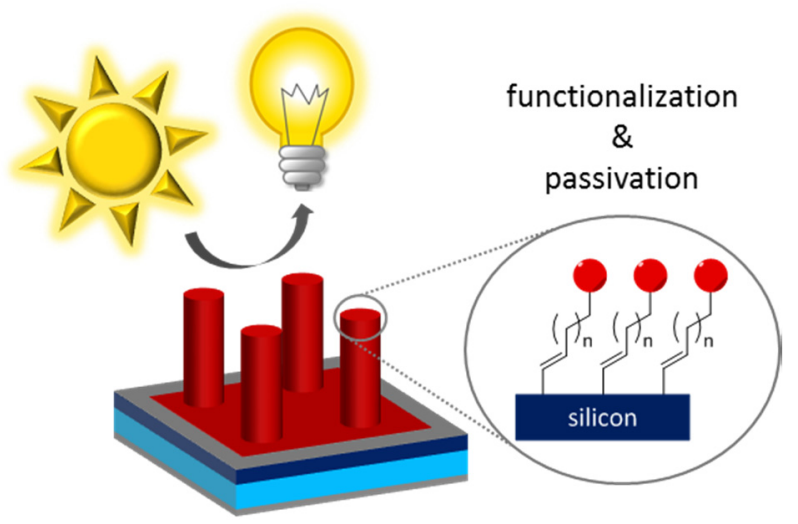

Part of this chapter has been published as: J. Veerbeek, N.J. Firet, W. Vijselaar, R. Elbersen, J.G.E. Gardeniers and J. Huskens, ACS Appl. Mater. Interfaces, 2017, 9, 413-421. 


\subsection{Introduction}

Silicon solar cells have gained increasing attention for electricity production, due to silicon's ease of fabrication, wide availability, and charge separation by using a $\mathrm{p} / \mathrm{n}$ junction. ${ }^{1-3}$ Because of the intermittent presence of sunlight, however, a steady power output is not feasible by photovoltaic cells only. Therefore, it is much more attractive to use sunlight to produce solar fuels, in which solar energy can be stored until needed. ${ }^{4,5}$ Examples of solar fuels include hydrogen from water splitting 6,7 or carbon-based fuels, such as $\mathrm{CO}, \mathrm{CH}_{4}$, or $\mathrm{CH}_{3} \mathrm{OH}$, from combining water splitting and $\mathrm{CO}_{2}{ }^{8}$ One of the envisioned solar-to-fuel devices consists of a tandem configuration, containing two semiconductors assembled with a membrane in between and catalysts on each side to assist water splitting. ${ }^{9,10}$ In this way, the solar cell converts sunlight into power, the catalysts use this power output to split water, and the evolved gases are kept separate by the membrane. To obtain the most efficient, integrated solar-to-fuel device, both an efficient solar cell and catalysts coupled to the surface are required. We focus here on devices that use silicon as one of the semiconductors, primarily for the hydrogen production side.

Structuring of silicon solar cells can increase the solar cell output by a higher surface area for light absorption and less reflection, e.g., using micropyramids or nano/micropillars with radial junctions. ${ }^{11-13} \mathrm{Nano} /$ microstructuring increases the surface area but inevitably also the amount of dangling bonds at the surface. This constitutes undesired recombination sites for electron-hole pairs, thus lowering the output of the solar cell. The negative effect of dangling bonds can be suppressed by applying an electrical passivation layer, ${ }^{14-16}$ e.g., an inorganic layer of silicon oxide, silicon nitride, or aluminum oxide. ${ }^{11,16-19}$

A solar-to-fuel device requires catalyst functionalization and passivation at the same time. For catalyst coupling to silicon, numerous literature examples have shown hydrogen production. ${ }^{10,18,20-22}$ In such an architecture, combining passivation and catalyst coupling can only be obtained in two steps, i.e., first catalyst deposition and then selectively applying a passivation layer by a bottom-up method that does not overgrow the catalyst ${ }^{23}$ or vice versa. ${ }^{18}$ Molecular monolayers, however, offer the solution to fulfill both surface passivation and subsequent catalyst coupling. Monolayer-forming adsorbates can be bound to silicon by direct $\mathrm{Si}-\mathrm{C}$ bonds in order to avoid the presence of silicon oxide as insulating layer and function as an electrical passivation layer. ${ }^{24-28} \mathrm{~A}$ few articles have reported the combination of passivation and secondary functionalization, ${ }^{29-31}$ but to our knowledge, this method has not been applied to silicon solar cells yet.

Here, we use organic molecular monolayers for electrical passivation and simultaneous functionalization of silicon solar cells. Covalent monolayer coupling has been achieved by hydrosilylation, which is a one-step reaction in which molecules with unsaturated carbon- 
carbon end groups are coupled to hydrogen-terminated silicon. ${ }^{32,33} 1$-Tetradecyne has been used to study the passivation effect on silicon solar cells by $\mathrm{J}-\mathrm{V}$ measurements (Section 3.2.1). Silicon solar cells have been fabricated with planar junctions in 2D substrates and radial junctions in 3D micropillars in order to assess the effect of $\mathrm{Si}$ microstructuring on the passivation effect. To test the versatility of this platform, the passivation effect was investigated on both $\mathrm{n}^{+} / \mathrm{p}$ and $\mathrm{p}^{+} / \mathrm{n}$ junctions. A monolayer of the dialkyne 1,8-nonadiyne has been studied to demonstrate that the passivation layers are still available for further functionalization (Section 3.2.2). As a proof of concept, a fluorescent dye has been coupled as model catalyst by the well-developed click chemistry. ${ }^{34}$

\subsection{Results and discussion}

Silicon solar cells with a junction were fabricated in 2D substrates and 3D micropillars (4 $\mu \mathrm{m}$ diameter, $6 \mu \mathrm{m}$ pitch, about $40 \mu \mathrm{m}$ length, Figure 3.1). Planar and pillared $\mathrm{Si}$ substrates were doped to create planar and radial junctions, respectively, according to a procedure reported before. ${ }^{35}$ Both $n^{+} / p$ junctions (defined as $p$-type base with phosphorus doping) and $\mathrm{p}^{+} / \mathrm{n}$ junctions (n-type base wafers doped with boron) were created, with a junction depth of approximately $1 \mu \mathrm{m}$.

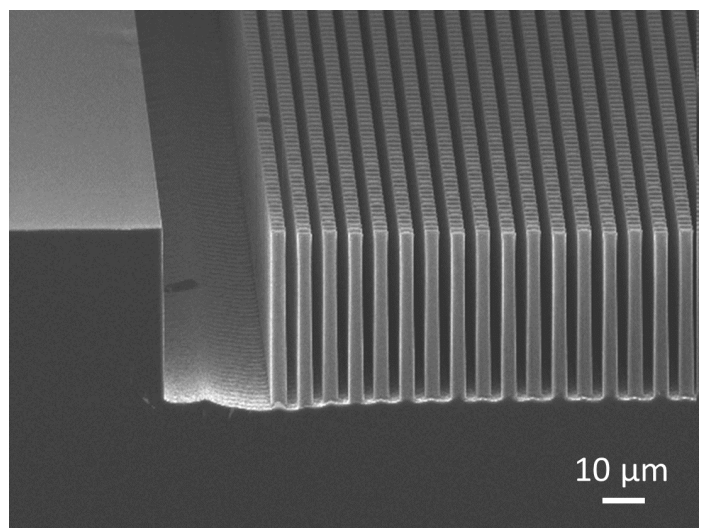

Figure 3.1. Cross-sectional high-resolution scanning electron microscopy (HR-SEM) image of a silicon micropillar array.

\subsubsection{Monolayer passivation}

In order to passivate the surface, monolayers were coupled to the solar cell substrates by a thermal hydrosilylation method in which chemical compounds with unsaturated end groups are grafted onto oxide-free silicon (Scheme 3.1). ${ }^{32,36}$ To that purpose, the silicon substrates were immersed in an aqueous $1 \%$ hydrofluoric acid (HF) solution to remove the native oxide layer and create a hydrogen-terminated surface. An alkyne-terminated molecule, i.e., 1-tetradecyne with a methyl end group, was coupled to hydrogen- 
terminated silicon to create a $\mathrm{Si}-\mathrm{C}$ bound monolayer. This molecule was chosen because of its hydrophobic nature, enabling easy proof of monolayer presence by contact angle measurements.

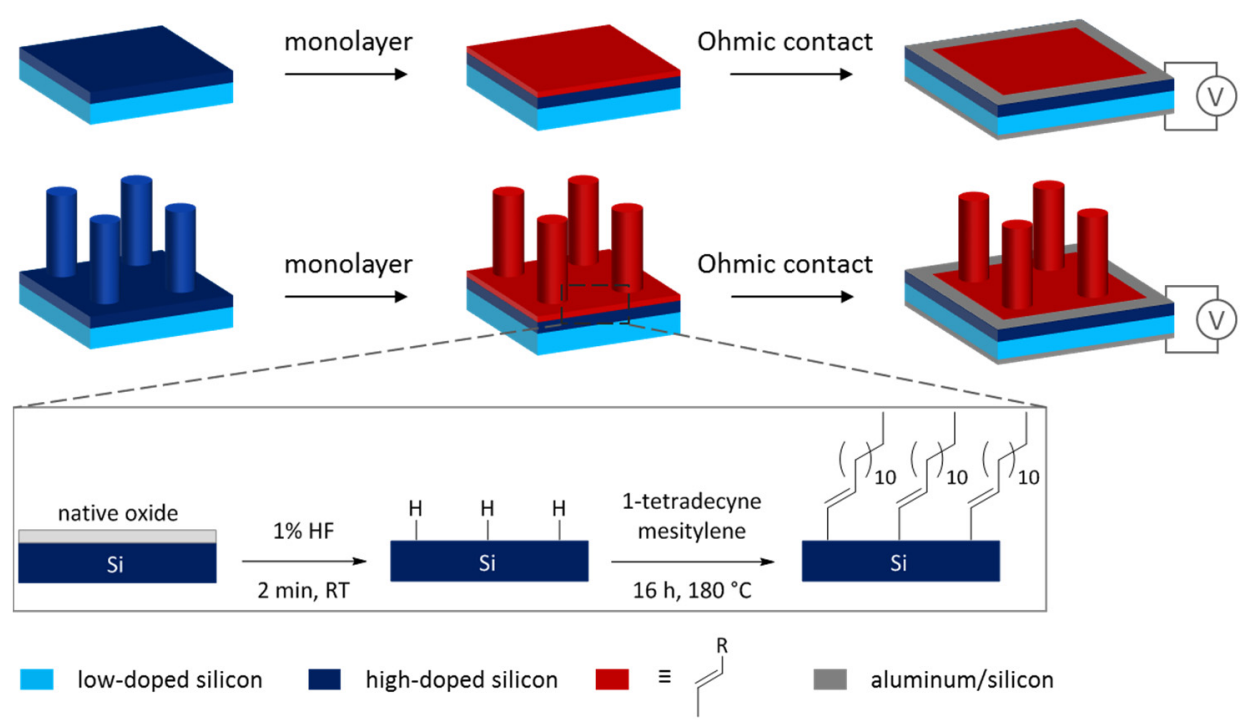

Scheme 3.1. Schematic representation of monolayer coupling to planar and radial junctions at planar and pillared Si substrates, respectively, by hydrosilylation. Aluminum contacts are created in order to assess the solar cell properties of the substrates.

The contact angle of hydrogen-terminated silicon was $42-44^{\circ}$ (Table 3.1) and increased upon hydrosilylation with 1-tetradecyne to $\sim 108^{\circ}$ for $\mathrm{n}^{+} / \mathrm{p}$ junctions and $\sim 95-98^{\circ}$ for $\mathrm{p}^{+} / \mathrm{n}$ junctions, as measured on the planar substrates or planar regions next to the pillar arrays. These values are characteristic for hydrophobic surfaces, thus indicating that the monolayer coupling was successful. The large difference in contact angle between $\mathrm{n}^{+}$and $\mathrm{p}^{+}$surfaces has been reported before for a visible light-induced hydrosilylation reaction ${ }^{37}$ and was attributed to the formation of more electron-hole pairs in $\mathrm{n}^{+}$substrates, which speeds up the radical chain mechanism. The control samples without a monolayer were processed through all steps, but their hydrosilylation step was mimicked with solvent only. For these control samples, the contact angle was $\sim 91-94^{\circ}$ for $\mathrm{n}^{+} / \mathrm{p}$ junctions and $\sim 84-87^{\circ}$ for $\mathrm{p}^{+} / \mathrm{n}$ junctions, which is inexplicably higher than the values of the hydrogenterminated samples.

Table 3.1. Static water contact angle values before and after monolayer formation.

$\begin{array}{lcccc} & 1 \% \mathrm{HF} & \text { Control } & \text { 1-Tetradecyne } & \text { 1,8-Nonadiyne } \\ \mathrm{n}^{+} / \mathrm{p} \text { planar } & 42.3^{\circ} \pm 0.8 & 94.1^{\circ} \pm 1.1 & 108.0^{\circ} \pm 2.6 & 76.0^{\circ} \pm 2.3 \\ \mathrm{n}^{+} / \mathrm{p} \text { radial } & & 91.4^{\circ} \pm 0.5 & 108.5^{\circ} \pm 0.4 & \\ \mathrm{p}^{+} / \mathrm{n} \text { planar } & 43.6^{\circ} \pm 2.3 & 87.1^{\circ} \pm 2.7 & 98.0^{\circ} \pm 0.5 & 82.6^{\circ} \pm 2.0 \\ \mathrm{p}^{+} / \mathrm{n} \text { radial } & & 84.8^{\circ} \pm 2.3 & 95.4^{\circ} \pm 0.8 & \end{array}$


Attenuated total reflection infrared spectroscopy (ATR-IR) spectra of dummy substrates, functionalized simultaneously with the solar cell samples, confirmed the presence of a 1-tetradecyne monolayer by disappearance of the $\mathrm{Si}-\mathrm{H}_{\mathrm{x}}$ stretching modes at $2112 \mathrm{~cm}^{-1}$ and the appearance of $\mathrm{CH}_{3}, \mathrm{CH}_{2}$ (antisymmetric), and $\mathrm{CH}_{2}$ (symmetric) peaks at 2961, 2922 , and $2853 \mathrm{~cm}^{-1}$, respectively (Figure 3.2). As expected, no peaks appeared in the spectra of the control samples, thus indicating that no monolayer was formed on these bare samples.

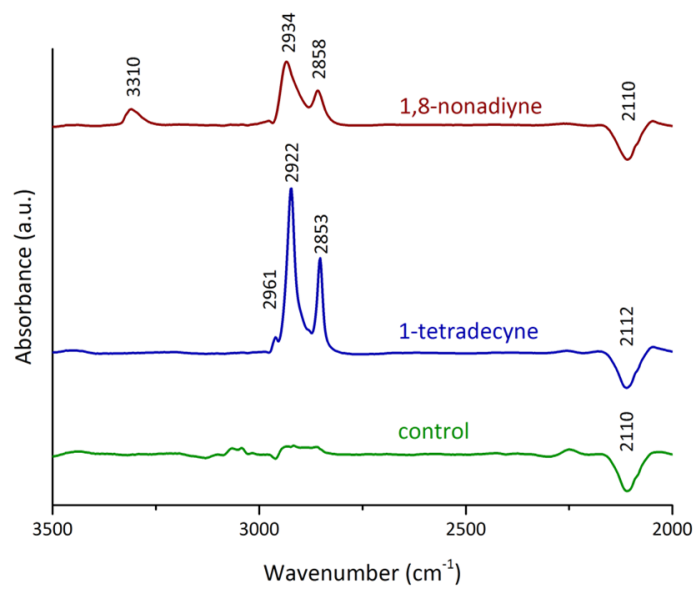

Figure 3.2. ATR-IR spectra of silicon substrates without functionalization (control) and functionalized with 1-tetradecyne or 1,8-nonadiyne, all referenced to an $\mathrm{H}$-terminated silicon sample.

In order to characterize the substrates as solar cells, Ohmic contacts of an aluminum/silicon alloy (99/1\%) were sputtered at the front and back side. The presence of a monolayer at the interface between the solar cell interface and the metal contact suppressed the conductance significantly, probably due to a poor contact between the aluminum and the silicon (data not shown). Therefore, to fabricate an Ohmic contact at the front side of the samples, the active solar cell area $\left(0.5 \times 0.5 \mathrm{~cm}^{2}\right)$ was first protected by photoresist masking. The 1-tetradecyne monolayer was then selectively removed outside the active solar cell areas by oxygen plasma, followed by removal of the resist. The thin silicon oxide layer induced by the oxygen plasma treatment was removed in $1 \% \mathrm{HF}$. The monolayers on the active solar cell areas were still intact after this treatment, as indicated by the high hydrophobicity on the planar junctions. Hereafter, aluminum/silicon alloy was sputtered outside the active/functionalized solar cell areas and on the back side of the samples. 
$J-V$ measurements were performed to investigate the effect of the monolayers on the solar cell performance. The efficiency $(\eta)$ of a solar cell can be calculated by Equation (1):

$$
\eta=\frac{V_{o c} J_{s c} F F}{P_{i n}}
$$

where $V_{o c}$ is the open-circuit voltage, $J_{s c}$ is the short-circuit current density, $F F$ is the fill factor, and $P_{\text {in }}$ is the input power $\left(100 \mathrm{~mW} / \mathrm{cm}^{2}, A M 1.5 \mathrm{G}\right)$. An ideal solar cell would show rectangular $J-V$ behavior, with $V_{o c}, J_{s c}$, and $F F$ as high as possible. FF mainly depends on two resistances, i.e., the shunt resistance $\left(R_{s h}\right)$ and the series resistance $\left(R_{s}\right) . R_{s h}$ is equal to the inverse slope of the $J-V$ curve at $J_{s c}$, which becomes higher when there are less leaking pathways for the current, as is achieved by more effective surface passivation. ${ }^{38,39}$ $R_{s}$ equals the inverse slope of the $J-V$ curve at $V_{o c}$ and reflects the resistance in the materials through which the current passes, and $R_{s}$ should be as low as possible.

$J-V$ measurements were performed on both planar and micropillar array samples with $\mathrm{n}^{+} / \mathrm{p}$ and $\mathrm{p}^{+} / \mathrm{n}$ junctions (Figure 3.3). The overall performance of planar $\mathrm{n}^{+} / \mathrm{p}$ (Figure 3.3a, Table 3.2) and $\mathrm{p}^{+} / \mathrm{n}$ junctions (Figure 3.3c, Table 3.3) did not change significantly after functionalization with a 1-tetradecyne monolayer. Internally, however, $R_{\text {sh }}$ increased by $15 \%$ and $4 \%$, respectively, thus indicating the expected positive effect of surface passivation. The $J-V$ response for micropillar arrays with radial $\mathrm{n}^{+} / \mathrm{p}$ (Figure $3.3 \mathrm{~b}$, Table 3.2) and $\mathrm{p}^{+} / \mathrm{n}$ junctions (Figure 3.3d, Table 3.3) improved significantly. Adding a monolayer enhanced all characteristic parameters, so that the efficiency increased from 8.7 to $9.9 \%$ for $\mathrm{n}^{+} / \mathrm{p}$ junctions and from $7.8 \%$ to $8.8 \%$ for $\mathrm{p}^{+} / \mathrm{n}$ junctions. Notably, $R_{\text {sh }}$ increased from 0.21 to $0.32 \mathrm{k} \Omega \cdot \mathrm{cm}^{2}$ ( $57 \%$ increase) for $\mathrm{n}^{+} / \mathrm{p}$ junctions and from 0.91 to $1.6 \mathrm{k} \Omega \cdot \mathrm{cm}^{2}$ (74\% increase) for $\mathrm{p}^{+} / \mathrm{n}$ junctions, thus confirming surface passivation by the monolayer. The improved efficiencies mainly originate from these differences in $R_{s h}$, whereas the $V_{o c}$ and $J_{s c}$ values changed only slightly, which shows that this passivation method results in an intrinsic change in material properties. 

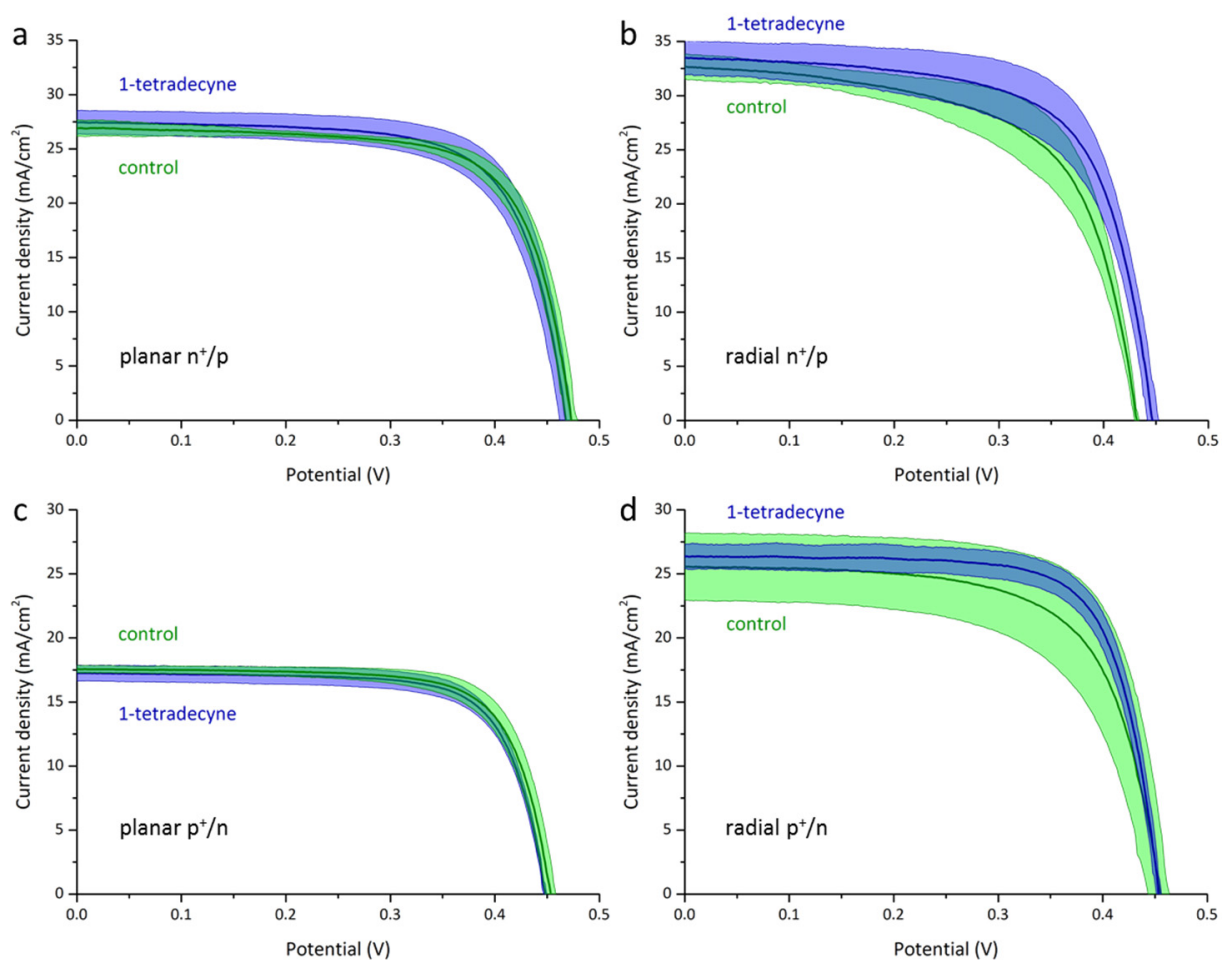

Figure 3.3. $J-V$ measurements of $S i$ samples with a) planar and b) radial $n^{+} / p$ junctions ( $p$-type base wafer) and c) planar and d) radial $\mathrm{p}^{+} / \mathrm{n}$ junctions (n-type base wafer) with and without a 1 -tetradecyne monolayer. The shaded areas indicate the $1 \sigma$-range around the average (at least four samples were analyzed for each configuration).

Table 3.2. $J-V$ characteristics of $\mathrm{n}^{+} / \mathrm{p}$ planar and radial junctions with and without a 1-tetradecyne monolayer.

\begin{tabular}{lcccccc} 
& \multicolumn{2}{c}{ Planar $\mathrm{n}^{+} / \mathrm{p}$ junctions } & \multicolumn{3}{c}{ Radial $\mathrm{n}^{+} / \mathrm{p}$ junctions } \\
& Control & 1-Tetradecyne & \pm & Control & 1-Tetradecyne & \pm \\
$R_{s}\left(\Omega \cdot \mathrm{cm}^{2}\right)$ & $1.5 \pm 0.1$ & $1.5 \pm 0.1$ & $-2 \% *$ & $1.6 \pm 0.1$ & $1.5 \pm 0.1$ & $9 \%$ \\
$R_{s h}\left(\mathrm{k} \Omega \cdot \mathrm{cm}^{2}\right)$ & $0.61 \pm 0.59$ & $0.69 \pm 0.02$ & $15 \%$ & $0.21 \pm 0.03$ & $0.32 \pm 0.08$ & $57 \%$ \\
$V_{o c}(\mathrm{~V})$ & $0.47 \pm 0.01$ & $0.47 \pm 0.01$ & $-1 \%$ & $0.43 \pm 0.01$ & $0.45 \pm 0.01$ & $4 \%$ \\
$J_{s c}\left(\mathrm{~mA} / \mathrm{cm}^{2}\right)$ & $26.9 \pm 0.8$ & $27.4 \pm 1.1$ & $2 \%$ & $32.7 \pm 1.2$ & $33.5 \pm 1.5$ & $2 \%$ \\
$F F(\%)$ & $71 \pm 4$ & $70 \pm 2$ & $-1 \%$ & $62 \pm 8$ & $66 \pm 4$ & $7 \%$ \\
$\eta(\%)$ & $9.0 \pm 0.4$ & $9.0 \pm 0.6$ & $0 \%$ & $8.7 \pm 1.0$ & $9.9 \pm 1.1$ & $14 \%$
\end{tabular}

$* R_{s}$ should be as low as possible, so a lower value is shown as positive difference 
Table 3.3. J-V characteristics of $\mathrm{p}^{+} / \mathrm{n}$ planar and radial junctions with and without a 1-tetradecyne monolayer.

\begin{tabular}{lcccccc} 
& \multicolumn{3}{c}{ Planar $\mathrm{p}^{+} / \mathrm{n}$ junctions } & \multicolumn{3}{c}{ Radial $\mathrm{p}^{+} / \mathrm{n}$ junctions } \\
& Control & 1 -Tetradecyne & \pm & Control & 1 -Tetradecyne & \pm \\
$R_{s}\left(\Omega \cdot \mathrm{cm}^{2}\right)$ & $2.2 \pm 0.1$ & $2.3 \pm 0.1$ & $-2 \% *$ & $1.9 \pm 0.3$ & $1.5 \pm 0.1$ & $27 \% *$ \\
$R_{s h}\left(\mathrm{k} \Omega \cdot \mathrm{cm}^{2}\right)$ & $1.39 \pm 0.22$ & $1.45 \pm 0.16$ & $4 \%$ & $0.91 \pm 0.11$ & $1.58 \pm 0.13$ & $74 \%$ \\
$V_{o c}(\mathrm{~V})$ & $0.45 \pm 0.01$ & $0.45 \pm 0.01$ & $-1 \%$ & $0.45 \pm 0.01$ & $0.45 \pm 0.01$ & $0 \%$ \\
$J_{s c}\left(\mathrm{~mA} / \mathrm{cm}^{2}\right)$ & $17.6 \pm 0.3$ & $17.3 \pm 0.6$ & $-2 \%$ & $25.6 \pm 2.6$ & $26.4 \pm 1.0$ & $3 \%$ \\
$F F(\%)$ & $74 \pm 3$ & $74 \pm 1$ & $0 \%$ & $67 \pm 6$ & $73 \pm 1$ & $9 \%$ \\
$\eta(\%)$ & $5.9 \pm 0.3$ & $5.7 \pm 0.3$ & $-3 \%$ & $7.8 \pm 1.5$ & $8.8 \pm 0.4$ & $12 \%$
\end{tabular}

$* R_{s}$ should be as low as possible, so a lower value is shown as positive difference

The passivation effect can also be derived from plotting the local ideality factor versus the potential (Figure 3.4), as extracted from the $J$ - $V$ behavior in the dark. The local ideality factor is expected to decrease when the number of defect sites is lowered upon applying a passivation layer. ${ }^{19,40,41}$ This effect was indeed observed for $\mathrm{n}^{+} / \mathrm{p}$ junctions, where the local ideality factor decreased for both planar and micropillar array samples with a 1-tetradecyne monolayer compared to unfunctionalized samples. For $\mathrm{p}^{+} / \mathrm{n}$ junctions, no difference in local ideality factor was observed, but the more pronounced differences in $R_{\text {sh }}$ already confirmed the passivation effect on these junctions.
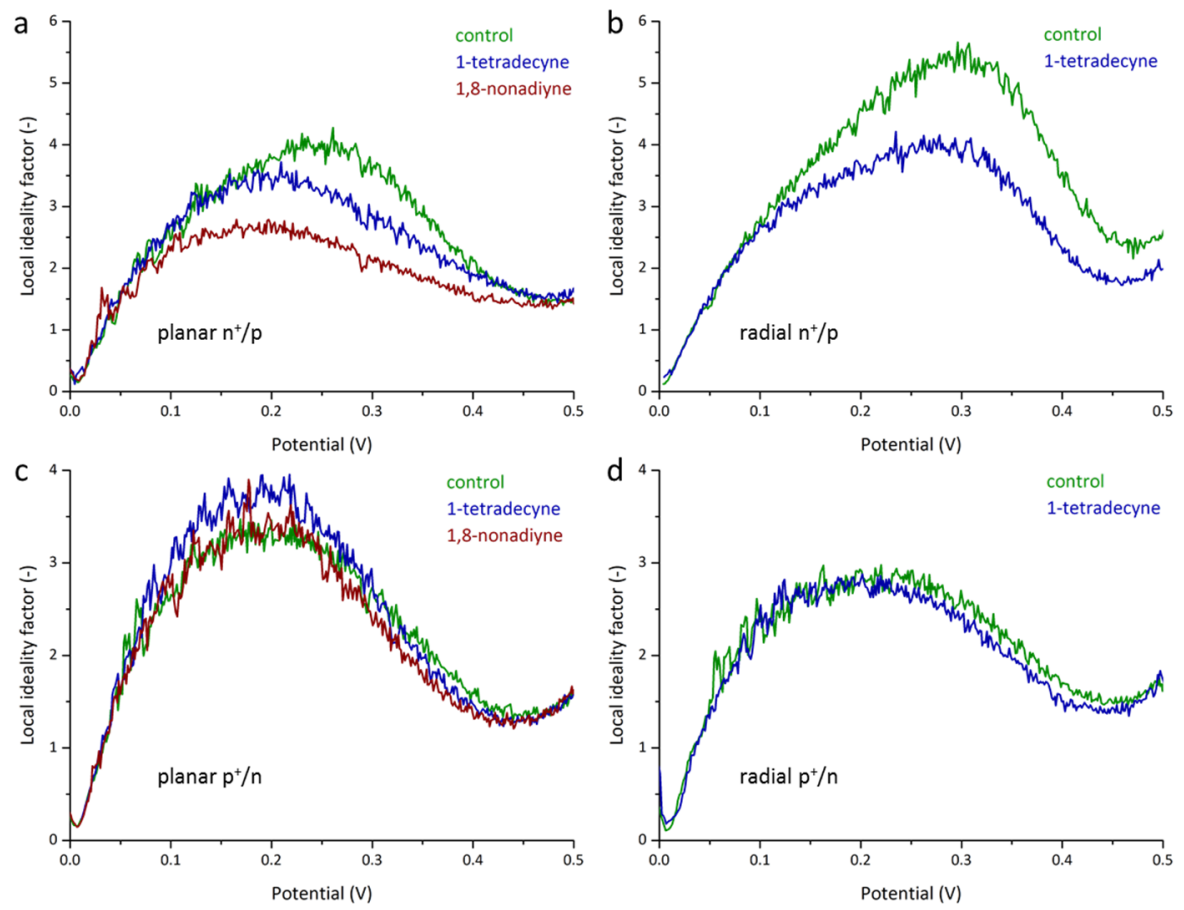

Figure 3.4. The local ideality factor for Si samples with a) planar and b) radial $\mathrm{n}^{+} / \mathrm{p}$ junctions (p-type base wafer) and c) planar and d) radial $\mathrm{p}^{+} / \mathrm{n}$ junctions ( $\mathrm{n}$-type base wafer) with and without a 1-tetradecyne or 1,8-nonadiyne monolayer. 
The passivation effect is most clearly visible on the micropillared solar cells, which can be explained by the difference in absolute surface area compared to a planar solar cell. With the micropillar layout used the total surface area has increased by a factor of $\sim 17$. As stated above, dangling bonds at the silicon surface provide undesired recombination sites for electron-hole pairs, which can be suppressed by coupling a molecular monolayer onto the surface. The micropillar solar cells contain more dangling bonds due to both their 3D structuring and the introduction of scallops during the pillar fabrication. ${ }^{11,42}$ This makes the effect of a molecular passivation layer more pronounced, thus emphasizing the need for passivation even more.

\subsubsection{Dual passivation and functionalization}

To allow both passivation and functionalization, an adsorbate with two functional groups, at the head and tail of the molecule, is required. A suitable choice is the dialkyne 1,8-nonadiyne, where one alkyne group can be coupled to the solar cell by hydrosilylation and the other alkyne group can be functionalized subsequently with an azide-containing molecule using the well-studied copper-catalyzed click chemistry ${ }^{34}$ or thiol-yne chemistry. ${ }^{43}$

Because we expected a very similar passivation effect of 1,8-nonadiyne compared to 1-tetradecyne, the electrical passivation effect of 1,8-nonadiyne was tested only on planar silicon solar cells. The hydrosilylation reaction was carried out in pure 1,8-nonadiyne in order to suppress back bending of the second alkyne group towards the hydrogen-terminated silicon surface. The contact angle of 1,8-nonadiyne-terminated surfaces was $76.0^{\circ}$ and $82.6^{\circ}$ for $\mathrm{n}^{+} / \mathrm{p}$ and $\mathrm{p}^{+} / \mathrm{n}$ planar junctions, respectively (Table 3.1). The latter value is similar to the literature, where $82^{\circ}$ was reported for a 1,8 -nonadiyne monolayer on $\mathrm{p}^{+}$silicon. ${ }^{44}$ ATR-IR further evidenced the monolayer formation by the disappearance of $\mathrm{Si}-\mathrm{H}_{\mathrm{x}}$ stretching modes at $2110 \mathrm{~cm}^{-1}$ and the appearance of $\mathrm{C} \equiv \mathrm{CH}, \mathrm{CH}_{2}$ (antisymmetric), and $\mathrm{CH}_{2}$ (symmetric) peaks at 3310, 2934, and $2858 \mathrm{~cm}^{-1}$, respectively (Figure 3.2), similar to spectra reported in the literature. ${ }^{43}$

After aluminum/silicon contact formation, $J-V$ measurements on the planar junctions did not show a significant difference of the efficiencies (Figure 3.5, Table 3.4). Looking at the individual parameters, however, $R_{\text {sh }}$ was improved, which indicates a passivated surface. The largest increase was observed for $\mathrm{n}^{+} / \mathrm{p}$ junctions (Figure 3.5a), similarly as described above for 1-tetradecyne. The values of $R_{s h}$ for the 1,8-nonadiyne-functionalized samples were higher than those obtained with 1-tetradecyne, indicating a better electrical passivation effect. This trend was also observed by a decrease of the local ideality factor (Figure 3.4), which was for planar $\mathrm{n}^{+} / \mathrm{p}$ junctions even close to ideal diode behavior, as featured by a local ideality factor below 2 under forward bias. ${ }^{40}$ 

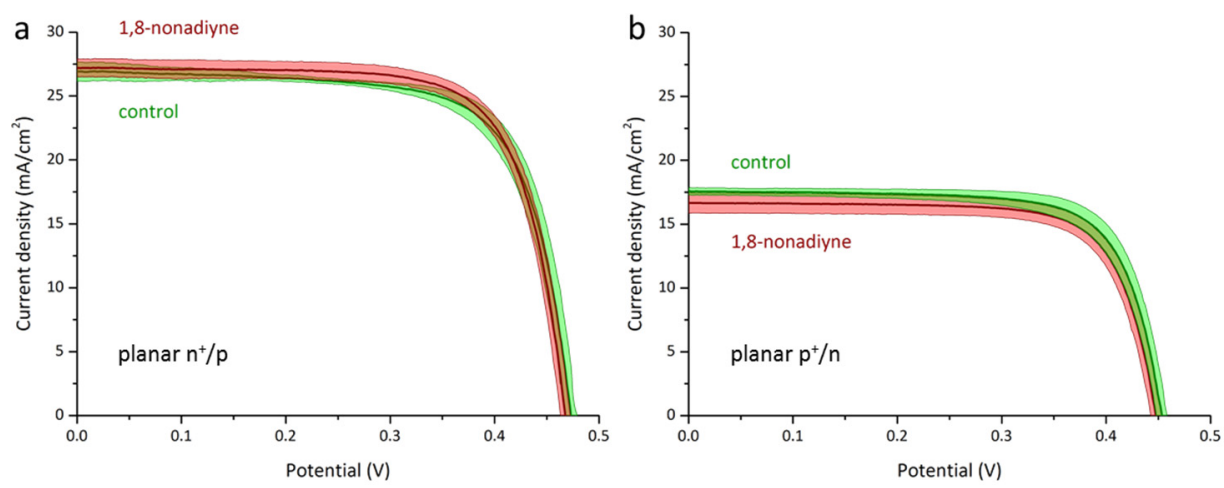

Figure 3.5. J-V measurements of planar a) $n^{+} / p$ junctions ( $p$-type base wafer) and $b$ ) $p^{+} / n$ junctions (n-type base wafer) with and without a 1,8-nonadiyne monolayer. The shaded areas indicate the $1 \sigma$-range around the average (at least four samples were analyzed for each configuration).

Table 3.4. J-V characteristics of planar $\mathrm{n}^{+} / \mathrm{p}$ and $\mathrm{p}^{+} / \mathrm{n}$ junctions with and without a 1,8-nonadiyne monolayer.

\begin{tabular}{lcccccc} 
& \multicolumn{3}{c}{ Planar $\mathrm{n}^{+} / \mathrm{p}$ junctions } & \multicolumn{3}{c}{ Planar $\mathrm{p}^{+} / \mathrm{n}$ junctions } \\
& Control & $1,8-$ Nonadiyne & \pm & Control & 1,8 -Nonadiyne & \pm \\
$R_{s}\left(\Omega \cdot \mathrm{cm}^{2}\right)$ & $1.5 \pm 0.1$ & $1.5 \pm 0.1$ & $4 \%^{*}$ & $2.2 \pm 0.1$ & $2.3 \pm 0.1$ & $-6 \% *$ \\
$R_{s h}\left(\mathrm{k} \Omega \cdot \mathrm{cm}^{2}\right)$ & $0.61 \pm 0.59$ & $1.74 \pm 0.01$ & $188 \%$ & $1.39 \pm 0.22$ & $1.65 \pm 0.14$ & $19 \%$ \\
$V_{o c}(\mathrm{~V})$ & $0.47 \pm 0.01$ & $0.47 \pm 0.01$ & $-1 \%$ & $0.45 \pm 0.01$ & $0.45 \pm 0.01$ & $-1 \%$ \\
$J_{s c}\left(\mathrm{~mA} / \mathrm{cm}^{2}\right)$ & $26.9 \pm 0.8$ & $27.2 \pm 0.7$ & $1 \%$ & $17.6 \pm 0.3$ & $16.7 \pm 0.8$ & $-5 \%$ \\
$F F(\%)$ & $71 \pm 4$ & $73 \pm 1$ & $3 \%$ & $74 \pm 3$ & $74 \pm 1$ & $1 \%$ \\
$\eta(\%)$ & $9.0 \pm 0.4$ & $9.3 \pm 0.2$ & $3 \%$ & $5.9 \pm 0.3$ & $5.5 \pm 0.3$ & $-5 \%$
\end{tabular}

${ }^{*} R_{s}$ should be as low as possible, so a lower value is shown as positive difference

As a proof of concept to confirm that the alkyne headgroup is still available for further functionalization, click chemistry was performed with an azide-functionalized dye as a model catalyst (Figure 3.6a). Microcontact printing was used to provide contrast with the background signal in fluorescence microscopy. A planar silicon substrate was successfully functionalized with azide-fluor 488 (Figure 3.6b, c). A line pattern is observed with a periodicity equal to that of the stamp used. X-ray photoelectron spectroscopy (XPS) of the monolayer after click chemistry showed the presence of the triazole moiety by two characteristic bands at 400 and $402 \mathrm{eV}$ in the N1s region (Figure 3.6d). Any physisorbed azide species would have appeared at $405 \mathrm{eV},{ }^{34}$ which was not observed in this spectrum (data not shown). Further evidence for the formation of the dye monolayer is the appearance of a peak at $288 \mathrm{eV}$ in the $\mathrm{C} 1 \mathrm{~s}$ spectrum owing to the introduction of carbonyl groups (Figure 3.6e). 

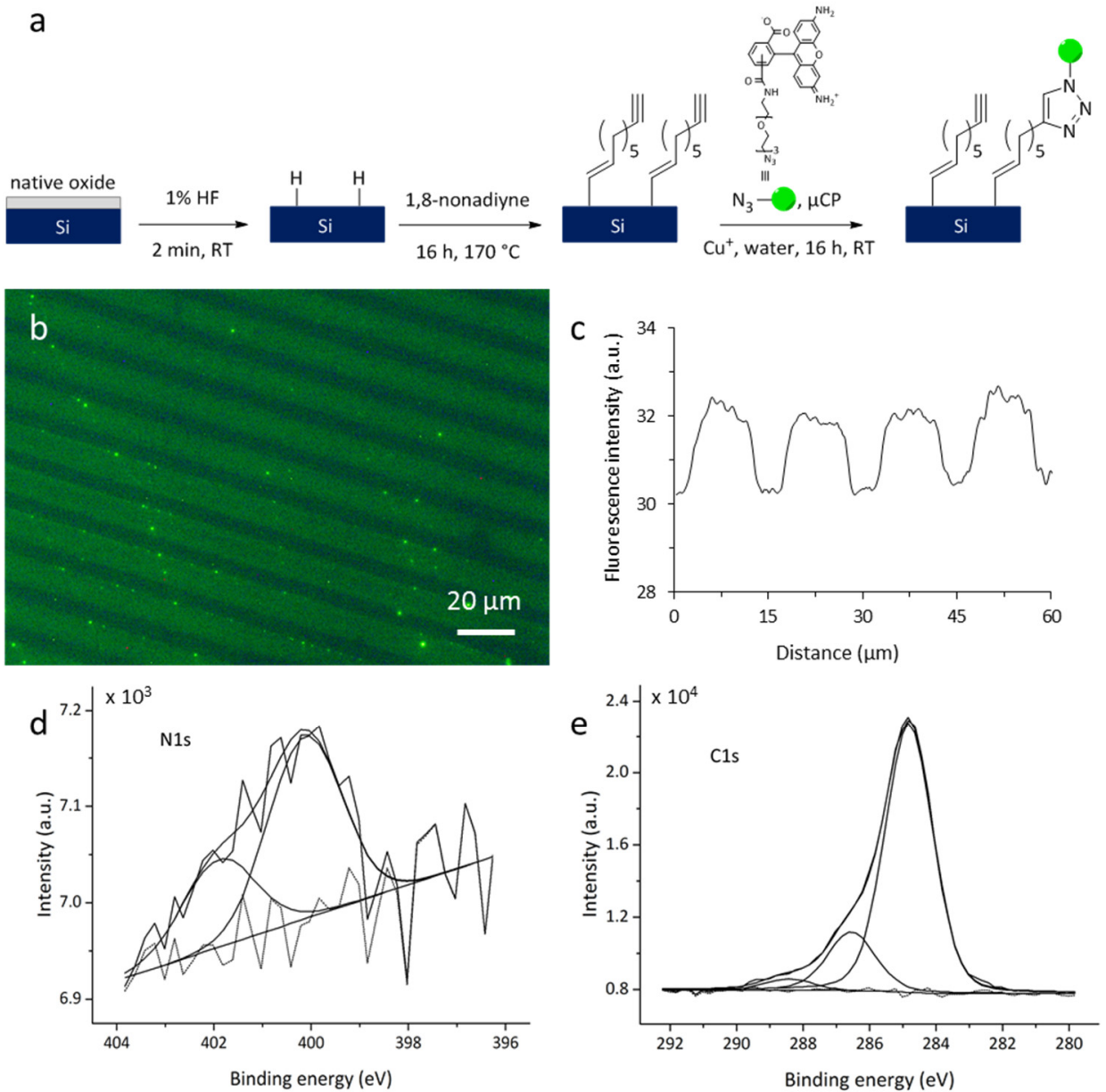

Figure 3.6. a) Schematic representation of 1,8-nonadiyne coupling to silicon by hydrosilylation and subsequent click chemistry with an azide-functionalized dye. b) Fluorescence microscopy image after microcontact printing, exposure time $10 \mathrm{~s}$, brightness and contrast increased by $30 \% \mathrm{c}$ ) Fluorescence intensity profile of the original image of (b). d,e) XPS spectra of d) the N1s region and e) the $\mathrm{C} 1 \mathrm{~s}$ region after microcontact printing, including deconvoluted signals.

\subsection{Conclusions}

In summary, we have shown the electrical passivation of silicon solar cells using molecular monolayers of 1-tetradecyne and 1,8-nonadiyne. The passivation effect was particularly observed by a substantial increase of the shunt resistance, which increased by $57 \%$ and $74 \%$ for $\mathrm{n}^{+} / \mathrm{p}$ and $\mathrm{p}^{+} / \mathrm{n}$ micropillar solar cells, respectively, when adding a monolayer of 1-tetradecyne. The molecular monolayers suppressed dangling bonds at the silicon surface, thus preventing recombination at those sites. This passivation effect was less pronounced in the case of planar junctions, because of their lower surface area and thus lower number of dangling bonds. Nevertheless, an increasing trend in shunt resistance 
was observed from control samples towards samples with monolayers of 1-tetradecyne and 1,8-nonadiyne. The latter monolayer was still available for further functionalization, as shown by coupling to a model catalyst. Since the length of the 1,8-nonadiyne is less than $1 \mathrm{~nm}$, we expect still a decent electrical contact between silicon and any catalyst bound on top of the monolayer, as indicated by the use of alkyl monolayers to prepare molecular junctions ${ }^{45,46}$ and the successful detection of redox-active groups on silicon bridged through alkyl monolayers. ${ }^{47,48}$ In the same manner, it should therefore be possible to functionalize silicon solar cells with an azide-functionalized photocatalyst towards a solar-to-fuel device. The resistance of the monolayer could be lowered by the use of conjugated molecules as passivation and functionalization layer. ${ }^{49,50}$

\subsection{Acknowledgments}

Nienke Firet is gratefully acknowledged for her work during the initial experiments, for her help, and for fruitful discussions. Wouter Vijselaar and Rick Elbersen are gratefully acknowledged for the fabrication of the (micropillar) solar cells and for fruitful discussions. Wouter is also kindly thanked for supplying the numerical model to fit the $\mathrm{J}-\mathrm{V}$ measurements. Carlo Nicosia is gratefully acknowledged for the synthesis of TBTA.

\subsection{Experimental section}

\subsubsection{Materials}

Silicon wafers ( $<100>$-oriented, $100 \mathrm{~mm}$ diameter, single side polished) were obtained from Okmetic (Finland) as p-type (boron, resistivity 5-10 $\Omega \cdot \mathrm{cm}$, thickness $525 \mu \mathrm{m}$ ) or n-type (phosphorus, 1-10 $\Omega \cdot \mathrm{cm}, 375 \mu \mathrm{m}$ ). For infrared spectroscopy, double side polished p-type silicon wafers were used with parameters equal to the single side polished p-type wafers. Mesitylene (>98\%, Sigma-Aldrich) and 1,8-nonadiyne (98\%, Sigma-Aldrich) were dried over molecular sieves (0.3 nm). Dichloromethane (99.7\%, Actu-All) was dried over anhydrous magnesium sulphate (Merck). Acetone (VLSI, BASF), acetonitrile (ACS grade, $\mathrm{CH}_{3} \mathrm{CN}$, Merck), azide-fluor 488 (>90\%, Sigma-Aldrich), buffered hydrogen fluoride (VLSI, BHF, 7:1, Technic France), ethanol (absolute, VWR), ethylenediaminetetraacetic acid disodium salt dihydrate (>99\%, EDTA, Sigma-Aldrich), hydrofluoric acid 1\% (aqueous, VLSI, Technic France), phosphate-buffered saline powder ( $\mathrm{pH} 7.4$, results in $10 \mathrm{mM}$ PBS with $0.138 \mathrm{M} \mathrm{NaCl}$, Sigma-Aldrich), photoresist AZ9260 (Merck Microchemicals), photoresist OiR 906-12 (Fujifilm), 2-propanol (VLSI, BASF), resist developer OPD 4262 (Fujifilm), 1-tetradecyne (>97\%, Sigma-Aldrich), tetrakis-(acetonitrile)copper(I) hexafluorophosphate $\left(\mathrm{Cu}(\mathrm{I})\left(\mathrm{CH}_{3} \mathrm{CN}\right)_{4} \mathrm{PF}_{6}\right.$, Sigma-Aldrich), and Tween-20 (Aldrich) were used as received. Tris-(benzyltriazolylmethyl)amine (TBTA) was synthesized according to a procedure from the literature. ${ }^{51}$ Hexane was obtained from a solvent purification system (MB SPS-800). Milli-Q water with a resistivity $>18 \mathrm{M} \Omega \cdot \mathrm{cm}$ was obtained from a 
Milli-Q Integral water purification system (Merck Millipore). Glassware used for the hydrosilylation reactions was dried overnight at $120^{\circ} \mathrm{C}$.

\subsubsection{Methods}

Fabrication of silicon micropillars. Silicon micropillars were fabricated as reported before ${ }^{35}$ but without the use of silicon dioxide as a hard mask (Scheme 3.2). In short, cleaned silicon substrates were covered with a $100 \mathrm{~nm}$ thick silicon-rich silicon nitride layer (SiRN) using low-pressure chemical vapor deposition (LPCVD). The SiRN layer was removed from the front side using reactive ion etching, leaving a protective SiRN layer at the back side. After cleaning, a photoresist layer (OiR 906-12) on the front side was patterned by standard photolithography, resulting in $0.5 \times 0.5 \mathrm{~cm}^{2}$ hexagonal arrays of dots (diameter $4 \mu \mathrm{m}$, spacing $2 \mu \mathrm{m}$, packing density $35 \%$ ) for each $2 \times 2 \mathrm{~cm}^{2}$ sample. This resist layer functioned as a mask during deep reactive ion etching of silicon using the Bosch process. This process resulted in micropillars with a length of approximately $40 \mu \mathrm{m}$, which were cleaned by an $\mathrm{O}_{2} / \mathrm{CF}_{4}$ plasma before further processing.

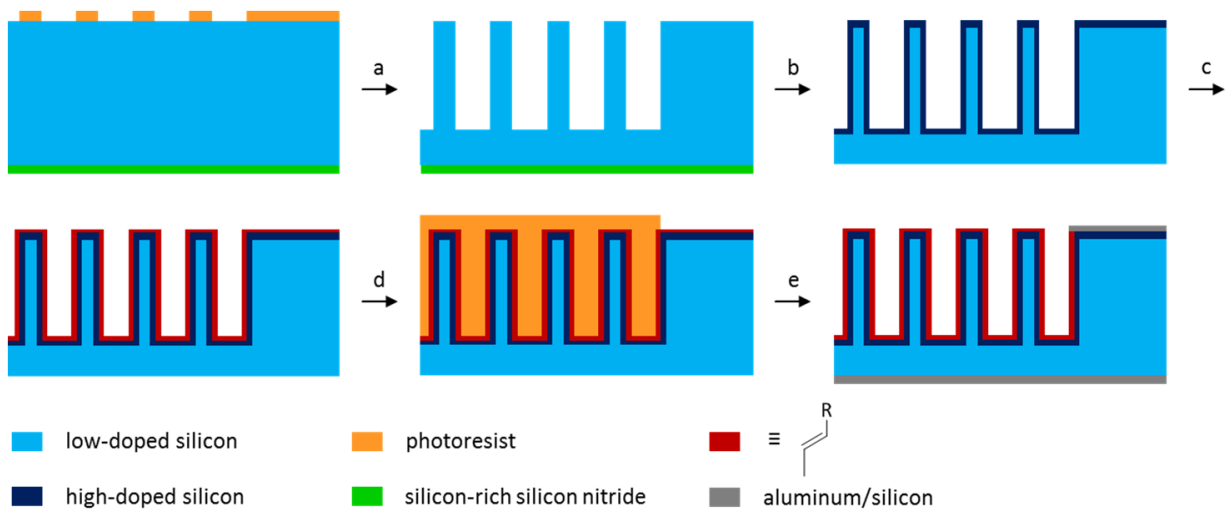

Scheme 3.2. Schematic fabrication process with a) micropillar fabrication using deep reactive ion etching, b) radial junction formation by dopant deposition and in-diffusion, followed by silicon nitride removal, c) organic monolayer coupling by hydrosilylation, d) photoresist coverage and photolithography, and e) monolayer removal outside the active solar cell areas and Ohmic contact formation at the front and back sides.

Junction fabrication. Planar silicon substrates and the silicon micropillars fabricated as described above were doped as reported before. ${ }^{35}$ Doping was performed on freshly cleaned substrates by depositing a dopant containing oxide layer, followed by a thermal drive-in step to diffuse the dopant into the silicon. In the case of $n^{+} / p$ junctions, $p$-type substrates were covered with a phosphorus oxide layer by LPCVD. Thermal diffusion was performed at $1050{ }^{\circ} \mathrm{C}$ for 15 min under nitrogen flow. Opposite $\mathrm{p}^{+} / \mathrm{n}$ junctions were created by doping $n$-type substrates with boron by solid-source dotation (SSD). A boron 
oxide $\left(\mathrm{B}_{2} \mathrm{O}_{5}\right)$ layer was grown from boron nitride source wafers and simultaneously diffused into the silicon at $1050{ }^{\circ} \mathrm{C}$ for $15 \mathrm{~min}$. After removal of the remaining $\mathrm{B}_{2} \mathrm{O}_{5}$ layer by $10 \mathrm{~min}$ immersion in $\mathrm{BHF}$, the wafers were further oxidized at $800{ }^{\circ} \mathrm{C}$ for $30 \mathrm{~min}$ to remove the Si-B bonds. The back side of these wafers was doped with phosphorus (similarly as described above) to create $\mathrm{n}^{+} \mathrm{Si}$, which was needed to create Ohmic contacts later on. In all cases, the SiRN protection layer was removed, and the samples were cleaned before further processing.

Monolayer coupling. To couple the alkyne-terminated molecules to the silicon substrates, first a hydrogen-terminated surface was created by 2 min immersion in an aqueous $1 \% \mathrm{HF}$ solution. Subsequently, the wafers were transferred into a full-wafer setup, equipped with a capillary as a nitrogen inlet and a reflux condenser, which was flushed with nitrogen for $10 \mathrm{~min}$. The adsorbate solution $(80 \mathrm{~mL})$, consisting of a $5 \% \mathrm{v} / \mathrm{v}$ solution of 1-tetradecyne in mesitylene or pure 1,8-nonadiyne, was added to the setup via a septum after degassing the solution by four freeze-pump-thaw cycles. The control samples were processed through the same steps as the samples with a monolayer, but the reaction step was performed in pure mesitylene, i.e., in the absence of adsorbate. The hydrosilylation reactions were performed overnight under continuous nitrogen flow at $180^{\circ} \mathrm{C}$ for 1 -tetradecyne, $170{ }^{\circ} \mathrm{C}$ for 1,8 -nonadiyne, and $180{ }^{\circ} \mathrm{C}$ for the control samples in pure mesitylene. The wafers were cleaned by immersion in hexane, rinsing with ethanol, 10 min ultrasonication in dichloromethane to remove any physisorbed material, and subsequently dried in a stream of nitrogen. The functionalized samples were sealed in a nitrogen glovebox and stored under nitrogen prior to characterization.

The monolayer was removed outside the active solar cell areas in order to make a proper Ohmic contact. Therefore, the active areas were first protected with photoresist. The planar junctions were protected by spin coating photoresist (OiR 906-12, $6000 \mathrm{rpm}, 30 \mathrm{~s}$ ) on top of the monolayer. The resist layer was baked at $95{ }^{\circ} \mathrm{C}$ for $90 \mathrm{~s}$, patterned using standard photolithography (3 S UV exposure), and immersed in resist developer (OPD 4262, 45 s). The micropillar arrays were protected by spin coating photoresist (AZ9260, $1000 \mathrm{rpm}, 30 \mathrm{~s}$ ) in between the pillars, overnight drying in a desiccator at $1 \times 10^{-3} \mathrm{mbar}$, photolithography ( 3 cycles of $10 \mathrm{~s}$ UV exposure and $10 \mathrm{~s}$ delay), and resist development (OPD 4262, $7 \mathrm{~min}$ ). In all cases, the wafers were exposed to oxygen plasma (Tepla 300E, $0.25 \mathrm{mbar}, 300 \mathrm{~W}$ ) for $2 \mathrm{~min}$ to remove the monolayer from the nonactive areas. On the planar junctions, the solar cell area was marked before resist removal in order to align the Ohmic contacts later on. The resist was removed by $15 \mathrm{~min}$ ultrasonication in acetone. Afterwards, the samples were rinsed with 2-propanol and dried under nitrogen. 
Click chemistry by microcontact printing. Poly(dimethylsiloxane) (PDMS) stamps were prepared by casting the precursor and curing agent (Sylgard 184, Dow Corning) at a 10:1 volume ratio onto a silicon master. Air bubbles were removed by vacuum for $30 \mathrm{~min}$, and the stamps were cured overnight at $60^{\circ} \mathrm{C}$. Before microcontact printing, the cut stamps (10 $\mu \mathrm{m}$ lines and $5 \mu \mathrm{m}$ spacing) were oxidized by oxygen plasma (power tuned to $40 \mathrm{~mA}$ ) for $30 \mathrm{~s}$. The stamps were inked with $75 \mu \mathrm{L}$ of azide-fluor 488 solution ( $2 \mathrm{mM}$ in $\mathrm{CH}_{3} \mathrm{CN}$ ) and $25 \mu \mathrm{L}$ of catalyst solution $\left(2 \mathrm{mM} \mathrm{Cu}(\mathrm{I})\left(\mathrm{CH}_{3} \mathrm{CN}\right)_{4} \mathrm{PF}_{6}\right.$ and $2 \mathrm{mM}$ TBTA in $\mathrm{CH}_{3} \mathrm{CN} /$ ethanol, ratio 2:1 v/v) for $4 \mathrm{~min}$. After drying in a stream of nitrogen, the stamp was brought into conformal contact with a planar substrate (functionalized with a monolayer of 1,8-nonadiyne) for $2 \mathrm{~h}$. Subsequently, the printed substrate was rinsed with acetonitrile and ethanol, and sonicated in PBS with $0.05 \% \mathrm{v} / \mathrm{v}$ Tween-20 for $1 \mathrm{~min}$. After rinsing with a $0.05 \% \mathrm{w} / \mathrm{v}$ EDTA solution in water to remove any copper traces, the substrate was dried under nitrogen.

Ohmic contact formation. Ohmic contacts were made in order to study the electrical properties of the formed junctions by recording $J-V$ curves. Native oxide was removed from the wafers by a $1 \% \mathrm{HF}$ dip, followed by sputtering a $1 \mu \mathrm{m}$ thick layer of aluminum/silicon alloy (99/1\% Al/Si, Oxford PL400, $500 \mathrm{~W}, 8: 24 \mathrm{~min}$ ) at the front side and $500 \mathrm{~nm}$ at the back side. At the front side, the active solar cell areas were protected by a stainless steel mask. The wafers were diced into single solar cells with $0.5 \times 0.5 \mathrm{~cm}^{2}$ active area and surrounding aluminum up to $2 \times 2 \mathrm{~cm}^{2}$.

\subsubsection{Equipment}

Contact angle measurements. Static contact angles were measured with Milli-Q water droplets on a Krüss G10 Contact Angle Measuring Instrument equipped with a CCD camera. Contact angle values were determined automatically by a drop shape analysis software. Contact angles were measured immediately after the hydrosilylation. At least three drops were measured and averaged. On the micropillar arrays, the contact angle was measured on the planar regions next to the pillars.

Attenuated total reflection infrared spectroscopy. Infrared spectra were measured on double side polished silicon wafer pieces treated similarly to the solar cell samples. Spectra were collected with a Bruker spectrometer (Vertex 70v) equipped with an attenuated total reflection accessory $(L=15 \mathrm{~mm})$ and MCT detector. The spectra were recorded with a resolution of $4 \mathrm{~cm}^{-1}$ and 1024 scans. Each spectrum was referenced to a hydrogen-terminated silicon sample. A spline baseline correction was applied.

$J-V$ measurements. Samples were positioned perpendicular to a $300 \mathrm{~W}$ xenon arc light source with a filter. The position of the samples with respect to the lamp was calibrated to 1 sun intensity (AM 1.5G). J-V curves were recorded with a VersaSTAT 4 potentiostat 
from -0.7 to $0.7 \mathrm{~V}$ at $0.2 \mathrm{~V} / \mathrm{s}$. For each setting at least four different samples were measured and averaged. The current densities were normalized to the sample area, instead of the actual surface area of the pillars. The series and shunt resistances were determined by linearly fitting the inverse slope of the average graph at the $x$-axis $\left(-10<J<10 \mathrm{~mA} / \mathrm{cm}^{2}\right)$ and $y$-axis $(-0.15<V<0.15 \mathrm{~V})$, respectively. The minimum and maximum series and shunt resistance were acquired by fitting the average graph \pm standard deviation, after which the standard deviation of the resistances was determined by one-fourth of the range.

The local ideality factor was extracted from the dark $J-V$ measurements by determining the slope of the $\ln (J)$ versus potential plot for each potential value according to Equation (2) and then averaging the local ideality factor over five points. The relation between current density (in the dark) and potential is given by the Shockley diode equation:

$$
\ln (J)=\ln \left(J_{0}\right)+\left(\frac{q}{n k T}\right) V
$$

where $J$ is the current density, $J_{0}$ is the dark saturation current density, $q$ is the absolute value of electron charge, $n$ is the ideality factor, $k$ is the Boltzmann constant, $T$ is the absolute temperature, and $V$ is the potential.

High-resolution scanning electron microscopy. HR-SEM images of cross sections of micropillar arrays were obtained with a FEI Sirion HR-SEM with a through-the-lens detector (TLD), operated at an acceleration voltage of $10 \mathrm{kV}$.

Fluorescence microscopy. Fluorescence microscopy images were acquired in air on an Olympus inverted research microscope IX71 equipped with a mercury burner U-RFL-T as light source and a digital Olympus DP70 camera. Blue excitation $\left(\lambda_{\text {ex }}=490-510 \mathrm{~nm}\right)$ and green emission $\left(\lambda_{e m}=520-550 \mathrm{~nm}\right.$ ) were filtered using a Chroma filter cube. Intensity profiles were obtained by averaging the images from three different places at the surface.

X-ray photoelectron spectroscopy. XPS measurements were performed on a Physical Electronics Quantera SXM setup equipped with an Al K $\alpha$ X-ray source (1486.6 eV). A detector angle of $45^{\circ}$ was used, and collected spectra were calibrated on the C1s peak at $284.8 \mathrm{eV}$.

\subsection{References}

1. M.D. Kelzenberg, D.B. Turner-Evans, M.C. Putnam, S.W. Boettcher, R.M. Briggs, J.Y. Baek, N.S. Lewis and H.A. Atwater, Energy Environ. Sci., 2011, 4, 866-871.

2. E. Garnett and P.D. Yang, Nano Lett., 2010, 10, 1082-1087.

3. T. Saga, NPG Asia Mater., 2010, 2, 96-102. 
4. M.G. Walter, E.L. Warren, J.R. McKone, S.W. Boettcher, Q.X. Mi, E.A. Santori and N.S. Lewis, Chem. Rev., 2010, 110, 6446-6473.

5. K. Sun, S. Shen, Y. Liang, P.E. Burrows, S.S. Mao and D. Wang, Chem. Rev., 2014, 114, 86628719.

6. P.P. Edwards, V.L. Kuznetsov, W.I.F. David and N.P. Brandon, Energy Policy, 2008, 36, 43564362.

7. J. Nowotny, C.C. Sorrell, L.R. Sheppard and T. Bak, Int. J. Hydrogen Energy, 2005, 30, 521-544.

8. W.G. Tu, Y. Zhou and Z.G. Zou, Adv. Mater., 2014, 26, 4607-4626.

9. J.M. Spurgeon, M.G. Walter, J.F. Zhou, P.A. Kohl and N.S. Lewis, Energy Environ. Sci., 2011, 4, 1772-1780.

10. Y.D. Hou, B.L. Abrams, P.C.K. Vesborg, M.E. Bjorketun, K. Herbst, L. Bech, A.M. Setti, C.D. Damsgaard, T. Pedersen, O. Hansen, J. Rossmeisl, S. Dahl, J.K. Norskov and I. Chorkendorff, Nat. Mater., 2011, 10, 434-438.

11. R. Elbersen, W. Vijselaar, R.M. Tiggelaar, H. Gardeniers and J. Huskens, Adv. Energy Mater., 2016, 6, 1501728.

12. Y.L. Li, Q. Chen, D.Y. He and J.S. Li, Nano Energy, 2014, 7, 10-24.

13. M.D. Kelzenberg, S.W. Boettcher, J.A. Petykiewicz, D.B. Turner-Evans, M.C. Putnam, E.L. Warren, J.M. Spurgeon, R.M. Briggs, N.S. Lewis and H.A. Atwater, Nat. Mater., 2010, 9, 239244.

14. M.V. Fernandez-Serra, C. Adessi and X. Blase, Nano Lett., 2006, 6, 2674-2678.

15. A.G. Aberle, Prog. Photovoltaics, 2000, 8, 473-487.

16. A.D. Mallorquí, E. Alarcón-Lladó, I.C. Mundet, A. Kiani, B. Demaurex, S. De Wolf, A. Menzel, M. Zacharias and A. Fontcuberta i Morral, Nano Res., 2015, 8, 673-681.

17. M.Z. Rahman, Renewable Sustainable Energy Rev., 2014, 30, 734-742.

18. Y.Q. Qu, L. Liao, R. Cheng, Y. Wang, Y.C. Lin, Y. Huang and X.F. Duan, Nano Lett., 2010, 10, 1941-1949.

19. W. Vijselaar, R. Elbersen, R.M. Tiggelaar, H. Gardeniers and J. Huskens, Adv. Energy Mater., 2016, 1601497.

20. I. Oh, J. Kye and S. Hwang, Nano Lett., 2012, 12, 298-302.

21. S.Y. Reece, J.A. Hamel, K. Sung, T.D. Jarvi, A.J. Esswein, J.J.H. Pijpers and D.G. Nocera, Science, 2011, 334, 645-648.

22. E.L. Warren, J.R. McKone, H.A. Atwater, H.B. Gray and N.S. Lewis, Energy Environ. Sci., 2012, 5, 9653-9661.

23. N.A. Ray, R.P. Van Duyne and P.C. Stair, J. Phys. Chem. C, 2012, 116, 7748-7756.

24. E.J. Nemanick, P.T. Hurley, L.J. Webb, D.W. Knapp, D.J. Michalak, B.S. Brunschwig and N.S. Lewis, J. Phys. Chem. B, 2006, 110, 14770-14778.

25. L.E. O'Leary, E. Johansson, B.S. Brunschwig and N.S. Lewis, J. Phys. Chem. B, 2010, 114, 1429814302.

26. A.B. Sieval, C.L. Huisman, A. Schonecker, F.M. Schuurmans, A.S.H. van der Heide, A. Goossens, W.C. Sinke, H. Zuilhof and E.J.R. Sudholter, J. Phys. Chem. B, 2003, 107, 6846-6852.

27. M.Y. Bashouti, J. Ristein, H. Haick and S. Christiansen, Hybrid Mater., 2014, 1, 2-14.

28. F. Zhang, D. Liu, Y. Zhang, H. Wei, T. Song and B. Sun, ACS Appl. Mater. Interfaces, 2013, 5, 4678-4684.

29. O. Assad, S.R. Puniredd, T. Stelzner, S. Christiansen and H. Haick, J. Am. Chem. Soc., 2008, 130, 17670-17671. 
30. S. Ciampi, P.K. Eggers, G. Le Saux, M. James, J.B. Harper and J.J. Gooding, Langmuir, 2009, 25, 2530-2539.

31. K.E. Plass, X.L. Liu, B.S. Brunschwig and N.S. Lewis, Chem. Mater., 2008, 20, 2228-2233.

32. A.B. Sieval, R. Linke, H. Zuilhof and E.J.R. Sudholter, Adv. Mater., 2000, 12, 1457-1460.

33. J.M. Buriak, Chem. Commun., 1999, 1051-1060.

34. S. Ciampi, T. Böcking, K.A. Kilian, M. James, J.B. Harper and J.J. Gooding, Langmuir, 2007, 23, 9320-9329.

35. R. Elbersen, R.M. Tiggelaar, A. Milbrat, G. Mul, H. Gardeniers and J. Huskens, Adv. Energy Mater., 2015, 5, 1401745.

36. L. Scheres, M. Giesbers and H. Zuilhof, Langmuir, 2010, 26, 10924-10929.

37. Q.Y. Sun, L.C.P.M. de Smet, B. van Lagen, M. Giesbers, P.C. Thune, J. van Engelenburg, F.A. de Wolf, H. Zuilhof and E.J.R. Sudholter, J. Am. Chem. Soc., 2005, 127, 2514-2523.

38. D. Zielke, J.H. Petermann, F. Werner, B. Veith, R. Brendel and J. Schmidt, Phys. Status Solidi $R R L, 2011,5,298-300$.

39. S. Banerjee and W.A. Anderson, Appl. Phys. Lett., 1986, 49, 38-40.

40. O. Breitenstein, J. Bauer, A. Lotnyk and J.M. Wagner, Superlattices Microstruct., 2009, 45, 182 189.

41. P.R. Pudasaini, D. Elam and A.A. Ayon, J. Phys. D: Appl. Phys., 2013, 46, 235104.

42. H.V. Jansen, M.J. de Boer, S. Unnikrishnan, M.C. Louwerse and M.C. Elwenspoek, J. Micromech. Microeng., 2009, 19, 033001.

43. N.S. Bhairamadgi, S. Gangarapu, M.A. Caipa Campos, J.M.J. Paulusse, C.J.M. van Rijn and H. Zuilhof, Langmuir, 2013, 29, 4535-4542.

44. M. James, T.A. Darwish, S. Ciampi, S.O. Sylvester, Z.M. Zhang, A. Ng, J.J. Gooding and T.L. Hanley, Soft Matter, 2011, 7, 5309-5318.

45. A. Salomon, T. Böcking, J. Gooding and D. Cahen, Nano Lett., 2006, 6, 2873-2876.

46. A. Vilan, O. Yaffe, A. Biller, A. Salomon, A. Kahn and D. Cahen, Adv. Mater., 2010, 22, 140-159.

47. A.G. Marrani, F. Cattaruzza, F. Decker, R. Zanoni, M. Cossi and M.F. Iozzi, J. Nanosci. Nanotechnol., 2010, 10, 2901-2907.

48. B. Fabre, Chem. Rev., 2016, 116, 4808-4849.

49. D.J. Wold, R. Haag, M.A. Rampi and C.D. Frisbie, J. Phys. Chem. B, 2002, 106, 2813-2816.

50. H.L. Yip, S.K. Hau, N.S. Baek, H. Ma and A.K.Y. Jen, Adv. Mater., 2008, 20, 2376-2382.

51. T.R. Chan, R. Hilgraf, K.B. Sharpless and V.V. Fokin, Org. Lett., 2004, 6, 2853-2855. 


\section{Chapter 4}

\section{Highly doped silicon nanowires by monolayer doping}

Controlling the doping concentration of silicon nanostructures is challenging. Here, we investigated three different monolayer doping techniques to obtain silicon nanowires with a high doping dose. These routes were based on conventional monolayer doping, starting from covalently bound dopant-containing molecules, or on monolayer contact doping, in which a source substrate coated with a monolayer of a carborane silane was the dopant source. As a third route, both techniques were combined to retain the benefits of conformal monolayer formation and the use of an external capping layer. These routes were used for doping fragile porous nanowires fabricated by metal-assisted chemical etching. The porosity was used to tune the total doping dose inside the nanowires, as measured by X-ray photoelectron spectroscopy and secondary ion mass spectrometry measurements. The higher the porosity, the higher the surface that was available for dopant-containing molecules, which in turn led to a higher doping dose. Slightly porous nanowires could be doped by all three routes, which resulted in highly doped nanowires with (projected areal) doping doses of $10^{14}-10^{15}$ boron atoms $/ \mathrm{cm}^{2}$ compared to $10^{12}$ atoms $/ \mathrm{cm}^{2}$ for a nonporous planar sample. Highly porous nanowires were not compatible with the conventional monolayer doping technique, but monolayer contact doping and the combined route resulted for these highly porous nanowires in tremendously high doping doses up to $10^{17}$ boron atoms $/ \mathrm{cm}^{2}$.

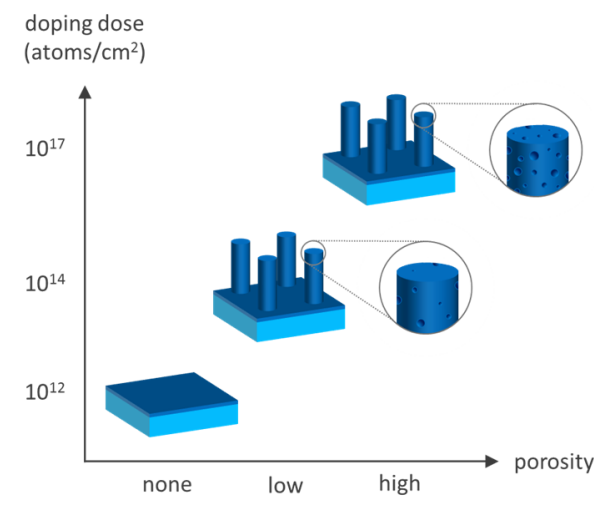

Part of this chapter has been published as: J. Veerbeek, L. Ye, W. Vijselaar, T. Kudernac, W.G. van der Wiel and J. Huskens, Nanoscale, 2017, 9, 2836-2844. 


\subsection{Introduction}

Silicon is an earth-abundant material that is commonly used for electronic and energy applications owing to its ease of structuring, ${ }^{1}$ versatile options for surface chemistry, ${ }^{2}$ and the large availability of doping methods. ${ }^{1}$ Silicon nanowire (Si NW) arrays are of high interest in sensing, photovoltaic, and electronic applications because of their onedimensional architecture and unique electrical, optical, and mechanical properties, and the availability of simple fabrication methods. ${ }^{3-5}$ These properties depend on the silicon doping level, ${ }^{6}$ thus achieving control over the doping dose is crucial for Si NW-based device fabrication.

Many different techniques exist to fabricate Si NWs, such as vapor-liquid-solid (VLS) growth, ${ }^{7,8}$ dry etching processes, ${ }^{9,10}$ and metal-assisted chemical etching (MACE). ${ }^{11,12}$ MACE has attracted attention during the last decade because it is a relatively inexpensive and simple approach. In the two-step method, a silicon wafer is covered with a metal film, mostly silver or gold, that promotes wet etching down into silicon when exposed to a $\mathrm{HF} / \mathrm{H}_{2} \mathrm{O}_{2}$ solution. The position of the nanowires can be defined using lithography, ${ }^{13-15}$ the diameter is determined by the gaps in the patterned metal film, and the length can be tuned by the etching time.

While many techniques are available to dope silicon, adding dopants to Si NWs is challenging. Most doping techniques that are applied to flat Si are destructive or not compatible with nonplanar structures. In situ doping is possible by VLS but suffers from inhomogeneous dopant distribution along the nanowire length. ${ }^{16}$ Doping Si NWs fabricated by MACE is even more challenging since these nanowires are partly porous, depending on the $\mathrm{H}_{2} \mathrm{O}_{2}$ concentration of the MACE solution and the doping concentration of the base wafer used. ${ }^{17-20}$ The techniques that have been used to dope MACE nanowires, i.e., chemical vapor deposition ${ }^{21-23}$ and spin-on dopants, ${ }^{24-26}$ lack uniformity and/or tunability of the doping dose. Epitaxial growth overcomes these problems but is only applicable to smooth nanowire surfaces. ${ }^{9}$ MACE applied to a Si wafer with a planar p-n junction does result in doped Si NWs, ${ }^{27-29}$ but does not allow for radial and shallow junction formation, nor does it give independent control over the doping dose and NW length as the initial doping should match the targeted NW length.

Monolayer doping (MLD) is suitable for creating high-quality, shallow junctions in silicon. ${ }^{30,31}$ It provides inherently uniform doping, also on 3D structures, since the initial formation of the self-assembled monolayer leads to a self-limiting coverage. The doping dose can be tuned downwards using mixed $M^{32}$ or upwards using dopant-rich molecules. ${ }^{33}$ Furthermore, ex situ doping is possible by monolayer contact doping (MLCD), in which the target substrate is doped by a source substrate that is functionalized with a monolayer of dopant molecules. ${ }^{34,35}$ MLD techniques have been used to dope $\mathrm{Si}$ 
NWs completely $\left(M L D,{ }^{30,36} \mathrm{MLCD}{ }^{34}\right)$ or with a parallel junction $\left(\mathrm{MLCD}^{35}\right)$, but, to our knowledge, the doping of MACE nanowires using MLD techniques has not yet been investigated.

Here, we report the use of three monolayer doping approaches, i.e., MLD (Section 4.2.2), MLCD (Section 4.2.3), and a combined method (Section 4.2.4), on Si NWs created by MACE. The influence of porosity on the final doping dose of the Si NWs is investigated. Wafers with different base doping concentrations were used to create NW arrays with different porosities (Section 4.2.1), as imaged by high-resolution scanning electron microscopy (HR-SEM). The coverage of dopant-containing molecules on these NWs is studied by contact angle measurements and X-ray photoelectron spectroscopy (XPS). Secondary ion mass spectrometry (SIMS) is used to determine the total doping dose after in-diffusion into the silicon lattice.

\subsection{Results and discussion}

Three different monolayer doping processes were tested on MACE-fabricated nanowires (Scheme 4.1). The first route employs the normal MLD process (Scheme 4.1a), which involves coupling of a monolayer of boron dopant-containing molecules to oxide-free, $\mathrm{H}$-terminated silicon by hydrosilylation. ${ }^{37,38}$ A silicon oxide capping layer is deposited to prevent the release of the molecules during the subsequent heating step. Rapid thermal annealing (RTA) ensures diffusion of the dopant atoms into the underlying silicon. The capping layer is removed in an aqueous buffered hydrogen fluoride (BHF) solution. Porous silicon NWs, however, are possibly incompatible with the oxide removal step, since BHF etches porous silicon. Therefore, MLCD (Scheme 4.1b) was tested, as it avoids capping layer formation and removal. This route uses a source substrate functionalized with boron dopant molecules, which is brought into contact with the silicon nanowire target substrate. RTA ensures boron in-diffusion into silicon. The use of a carborane silane molecule, which contains 10 boron atoms per molecule, can lead to higher doping levels. ${ }^{33}$

Because it is unknown whether the dopant is distributed conformally along the length of the nanowires in the MLCD process (Scheme 4.1b), a combination of MLD and MLCD was also tried (Scheme 4.1c). In this process, a monolayer is formed on the H-terminated nanowire surface by hydrosilylation as is done in the normal MLD process, followed by capping using a dummy wafer. While porous silicon is etched by BHF, it is compatible with the short $1 \% \mathrm{HF}$ dip required for the hydrosilylation reaction. ${ }^{39,40}$ Instead of capping the monolayer on the target substrate using silicon oxide which would require HF etching, a dummy substrate with silicon oxide is placed on top of the nanowire substrate as is normally done in MLCD. In this way, the benefits of direct and conformal monolayer formation on the target and the use of an external capping substrate are combined. 


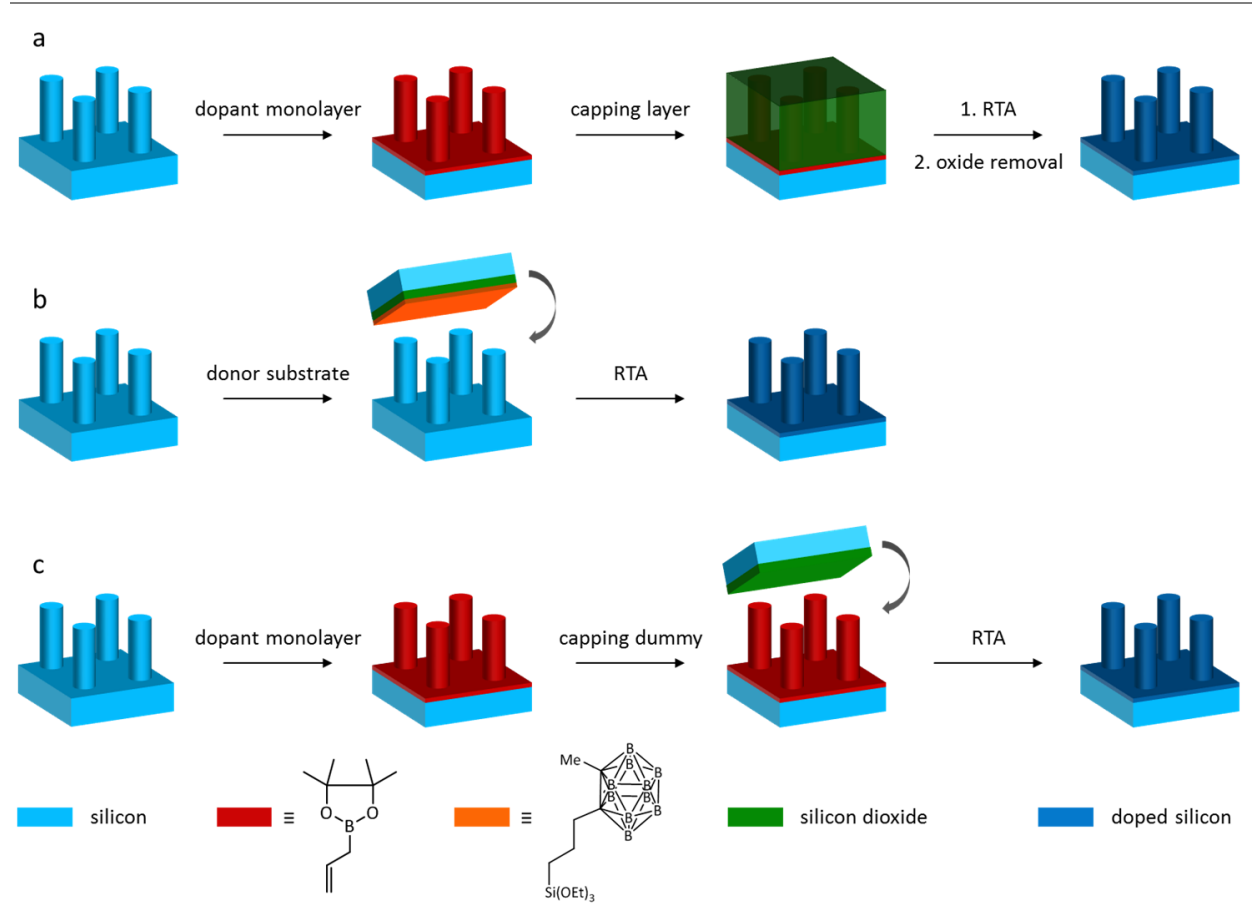

Scheme 4.1. Schematic illustration of the three different monolayer doping processes tested on MACE-fabricated silicon nanowire substrates, including a) MLD, b) MLCD, and c) MLD with external capping layer (RTA = rapid thermal annealing).

\subsubsection{Nanowire synthesis}

Nanowires were fabricated by nanolithography, i.e., either nanoimprint lithography or nanosphere lithography, and subsequent MACE. ${ }^{13,18,41}$ Most samples were created using nanosphere lithography (Scheme 4.2), where a close-packed array of polystyrene (PS) spheres was formed on a cleaned silicon substrate (Figure 4.1a). The diameter of the spheres was $447 \mathrm{~nm}$ in the dried state, which was decreased to about $280 \mathrm{~nm}$ by oxygen reactive ion etching (Figure 4.1b). Subsequently, a $40 \mathrm{~nm}$ silver layer was sputtered on top (Figure 4.1c), and lift-off of the polystyrene spheres was achieved in dichloromethane, resulting in a silver layer with gaps (Figure 4.1d). Exposing the substrate to an aqueous $\mathrm{HF} / \mathrm{H}_{2} \mathrm{O}_{2}$ solution caused wet etching of the silicon substrate underneath the silver layer. After etching of the resulting wires to the desired length, the silver layer was removed in $\mathrm{HNO}_{3}$ to yield clean silicon nanowire arrays. 


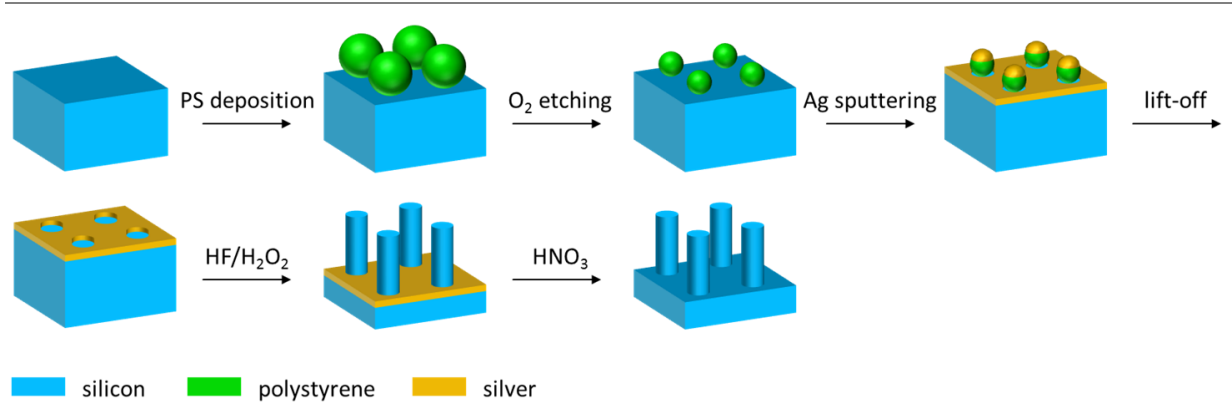

Scheme 4.2. Schematic illustration of the fabrication of Si NWs by combining nanosphere lithography and MACE.
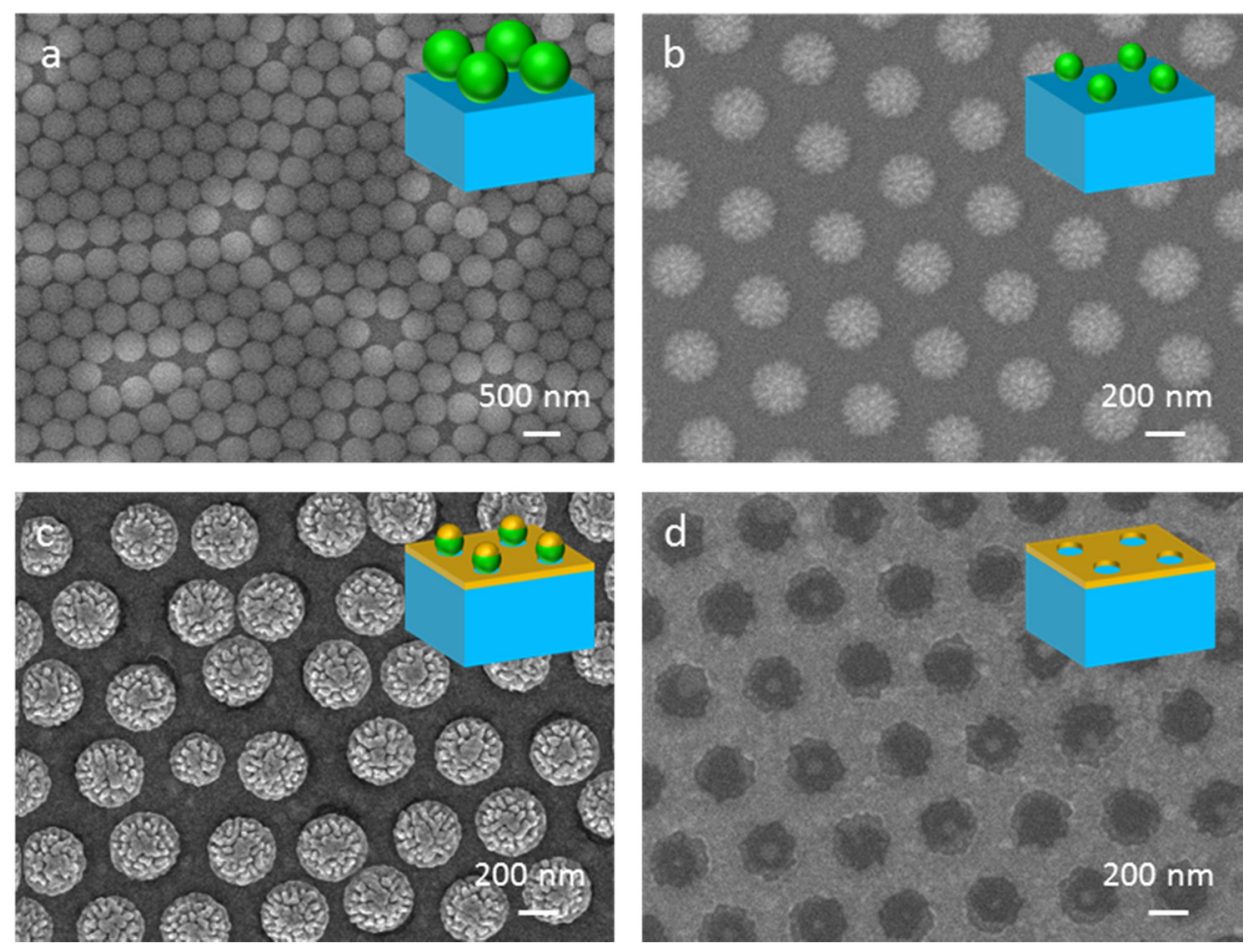

Figure 4.1. Top-view HR-SEM images of samples resulting from nanosphere lithography, including a) close-packed array formation of polystyrene spheres, b) diameter decrease by oxygen reactiveion etching, c) silver sputtering on top, and d) lift-off of polystyrene spheres.

The nanowires were prepared from p-type $(5-10 \Omega \cdot \mathrm{cm})$, n-type $(1-10 \Omega \cdot \mathrm{cm})$, and $\mathrm{n}^{++}(0.0012-0.0015 \Omega \cdot \mathrm{cm})$ silicon substrates. The fabrication process was equal for the different base wafers, but the etching rate was higher for the $\mathrm{n}^{++}$samples. All wires were tuned to be about $300 \mathrm{~nm}$ in diameter and $1 \mu \mathrm{m}$ in length (Figure 4.2). HR-SEM images showed a low porosity at the top of the p-type and n-type samples. In contrast, the 
$\mathrm{n}^{++}$samples were highly porous including a porous layer in the underlying Si substrate of $1.8 \mu \mathrm{m}$ underneath the nanowires, with a pore size of a few to $10 \mathrm{~nm}$.
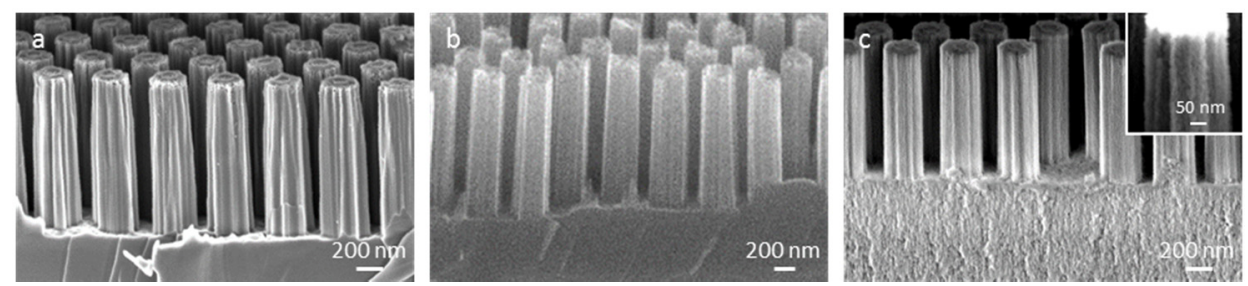

Figure 4.2. Cross-sectional HR-SEM images of cleaned Si NWs from a) p-type, b) n-type, and c) $\mathrm{n}^{++}$substrates (inset: zoom-in with pores shown as dark spots).

\subsubsection{Monolayer doping}

The usual MLD process (Scheme 4.1a) was tested on these nanowires. Boron dopant molecules (allylboronic acid pinacol ester) were coupled covalently to hydrogenterminated Si NWs created upon a short HF dip. Contact angle measurements on an intrinsic dummy substrate, functionalized simultaneously with the nanowire samples, indicated monolayer formation by a value of $73.6^{\circ} \pm 0.9$ comparable to a value reported before. $^{32}$ XPS showed successful monolayer coupling on $\mathrm{p}$-type and $\mathrm{n}^{++}$nanowires, as evidenced by the presence of boron (Table 4.1). The amount of boron on the highly porous $\mathrm{n}^{++}$nanowires is significantly higher than on the $\mathrm{p}$-type nanowires and on a planar intrinsic silicon substrate measured before. ${ }^{32}$ This is in correspondence with the high porosity detected in HR-SEM, resulting in a larger monolayer coverage for the $\mathrm{n}^{++}$samples. The $\mathrm{B} / \mathrm{C}$ ratio approaches the theoretical value of $0.11\left(\mathrm{C}_{9} \mathrm{H}_{17} \mathrm{O}_{2} \mathrm{~B}\right)$ on the $\mathrm{n}^{++}$nanowires. The lower value for the $\mathrm{p}$-type nanowires is probably due to adventitious carbon, as observed before on a planar sample. ${ }^{32}$

Table 4.1. Relative boron and carbon concentrations from XPS measurements on surfaces modified with monolayers of allylboronic acid pinacol ester.

$\begin{array}{llll}\text { Sample } & \mathrm{B}(\%) & \mathrm{C}(\%) & \mathrm{B} / \mathrm{C} \\ \text { p nanowires } & 0.68 \pm 0.32 & 25.5 \pm 0.8 & 0.027 \pm 0.013 \\ \mathrm{n}^{++} \text {nanowires } & 3.92 \pm 0.56^{*} & 40.2 \pm 0.8 & 0.098 \pm 0.014 \\ \text { i planar }^{32} & 1.09 \pm 0.10 & 34.1 \pm 2.0 & 0.032 \pm 0.003\end{array}$

$* p<0.01$ compared to either $p$ nanowires or i planar (Student's $t$-test, $n=4$ )

To test the influence of the amount of the dopant on junction formation, the boron dopants were diffused into silicon by depositing a $\mathrm{SiO}_{2}$ capping layer followed by RTA. MLD was successfully applied to the $p$ - and n-type nanowires. HR-SEM images showed no change in shape before and after MLD (Figure 4.3a, b). In contrast, the highly porous $\mathrm{n}^{++}$nanowires were etched away in this process (Figure 4.3c). This appeared to be caused 
by the BHF step, as tested on an undoped $\mathrm{n}^{++}$control sample with nanowires (data not shown). Consequently, the normal MLD process cannot be performed on $\mathrm{n}^{++}$nanowires.
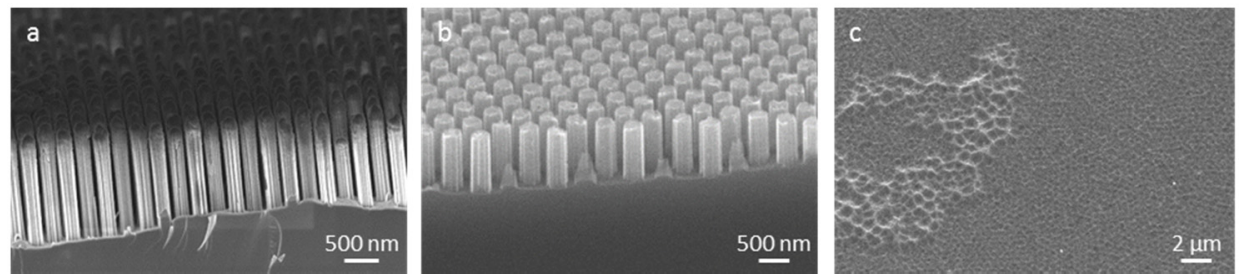

Figure 4.3. a, b) Cross-sectional and c) top-view HR-SEM images of Si NWs after MLD on a) p-type, b) n-type, and c) $n^{++}$substrates.

SIMS (Figure 4.4, Table 4.2) was used to measure the amount of boron after MLD on $\mathrm{p}$ - and n-type nanowires. The peak concentrations were about $10^{19}$ atoms $/ \mathrm{cm}^{3}$, which are comparable to the values reported before for boron-doped planar samples. ${ }^{32}$ The total boron dose, per projected surface area, was determined from the area under the concentration vs. depth graph (Figure 4.4) and amounted to $7.3 \times 10^{14}$ atoms $/ \mathrm{cm}^{2}$ for p-type nanowires, $1.6 \times 10^{14}$ atoms $/ \mathrm{cm}^{2}$ for n-type nanowires, and $2.9 \times 10^{12}$ atoms $/ \mathrm{cm}^{2}$ for a planar intrinsic sample. The total boron dose for the nanowire samples is thus two orders of magnitude higher than for the planar sample. It should be noted that the base concentration of the p-type nanowires is $\sim 10^{15}$ atoms $/ \mathrm{cm}^{3}$ (i.e., $10^{11}$ atoms $/ \mathrm{cm}^{2}$ for $1 \mu \mathrm{m}$ depth) and thus does not contribute significantly to the total amount of boron. No conclusions can be drawn about the distribution of boron atoms along the nanowire length, since the voids in between the nanowires result in random surface bombardments during the SIMS measurements.

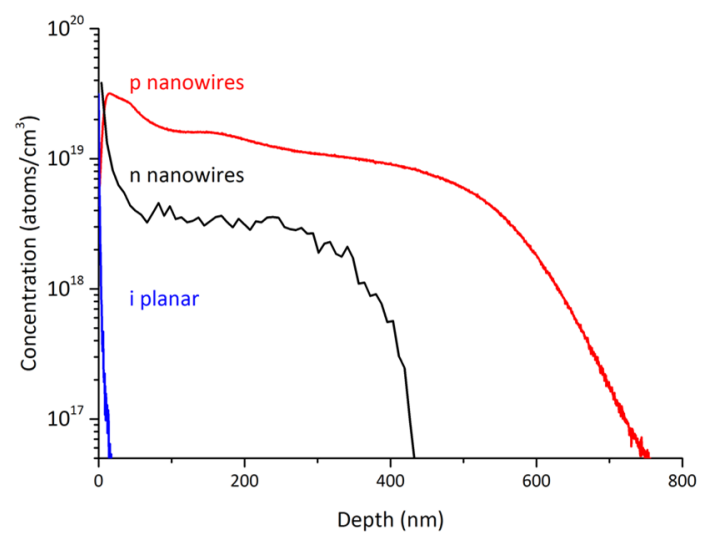

Figure 4.4. SIMS depth profiles of boron dopant atoms in p-type nanowires, n-type nanowires, and an intrinsic planar sample after MLD. 
Table 4.2. Overview of total boron doses in atoms $/ \mathrm{cm}^{2}$ as determined by SIMS.

$\begin{array}{llll}\text { Sample } & \text { MLD (a) } & \text { MLCD (b)* } & \text { MLD \& MLCD (c) } \\ \text { i planar } & 2.9 \times 10^{12} & 4.4 \times 10^{13} \text {, ref. } 42 & \\ \text { p nanowires } & 7.3 \times 10^{14} & 5.9 \times 10^{15} & 1.9 \times 10^{15} \\ \mathrm{n} \text { nanowires } & 1.6 \times 10^{14} & & \\ \mathrm{n}^{++} \text {nanowires } & \text { N/A* } & 8.1 \times 10^{15} & 1.4 \times 10^{17} \\ { }^{*} \text { Carborane molecules used with 10 boron atoms/molecule } & \\ \text { ** The } \mathrm{n}^{++} \text {nanowires were not compatible with the MLD process }\end{array}$

The observed two orders higher doping dose for the nanowires is larger than would have been expected for smooth, nonporous nanowires. The theoretical total surface area of the nanowire samples is just 6.5 times higher than that of a planar surface (based on nanowires with a diameter of $300 \mathrm{~nm}$, a length of $1 \mu \mathrm{m}$, and a pitch of $447 \mathrm{~nm}$ ). Surface roughness and a noncylindrical nanowire shape do increase the total surface area, while packing defects could slightly decrease the amount of nanowires present. In any case, the increase in total doping dose from planar to nanowire samples cannot be explained solely by the geometry.

We hypothesize that the porosity induced by MACE, even in lowly doped base wafers, offers a larger area for the covalent attachment of molecules in the MLD step and for in-diffusion during RTA, both of which enhance the total doping dose. Whereas most literature reports conclude that base wafers with a low doping concentration $(1-10 \Omega \cdot \mathrm{cm})$ lead to solid nanowires, ${ }^{19,20}$ Geyer et al. ${ }^{18}$ have concluded that MACE always results in a porous silicon layer. The thickness of this porous layer depends on the doping concentration of the base wafer. For doping levels lower than $10^{18}$ atoms $/ \mathrm{cm}^{3}$ $(>0.02 \Omega \cdot \mathrm{cm})$, a porous layer of about $100 \mathrm{~nm}$ was observed using transmission electron microscopy. Base wafers with more than $10^{18}$ atoms $/ \mathrm{cm}^{3}$ led to a porous silicon layer of several micrometers. Since we are using base wafers with a low doping concentration, the $\mathrm{p}$ - and n-type nanowires are expected to be porous with pores too small to be detected in HR-SEM. We therefore attribute the higher doping dose of the $p$ - and n-type nanowires to the porosity of the NWs. We note that the enhanced uptake and binding of the boron adsorbate after monolayer formation is not ( $p$-type wires) or less ( $\mathrm{n}^{++}$wires) visible by XPS (see Table 4.1), which is attributed to the surface sensitivity of XPS of only a few $\mathrm{nm}$ which obscures dopants present deeper inside the NWs.

\subsubsection{Monolayer contact doping}

As stated above, highly porous $\mathrm{n}^{++}$nanowires were not compatible with the BHF step of the MLD process. Therefore, MLCD was tested, in which a source substrate with a dopantcontaining monolayer is brought into contact with the target substrate (Scheme 4.1b). Here, we used a carborane silane as the monolayer on a silicon oxide source substrate, 
thus making use of the easier formation of silane layers compared to hydrosilylation and an oxide layer underneath that suppresses diffusion into the source substrate. ${ }^{42}$ Both p-type and $\mathrm{n}^{++}$nanowires were used as target samples. After the RTA step, HR-SEM images (Figure 4.5a) showed that the p-type nanowires were still intact. However, in the case of the $\mathrm{n}^{++}$nanowires, the piranha cleaning (for removal of carbon residuals) and $1 \% \mathrm{HF}$ dip (for native oxide removal) resulted in bundling of the nanowires and cracking of the porous layer underneath (Figure 4.5b), and this sample was therefore not further studied. Instead, performing only the RTA step, without the preceding cleaning and oxide removal steps, did not damage the $\mathrm{n}^{++}$nanowires (Figure 4.5c).
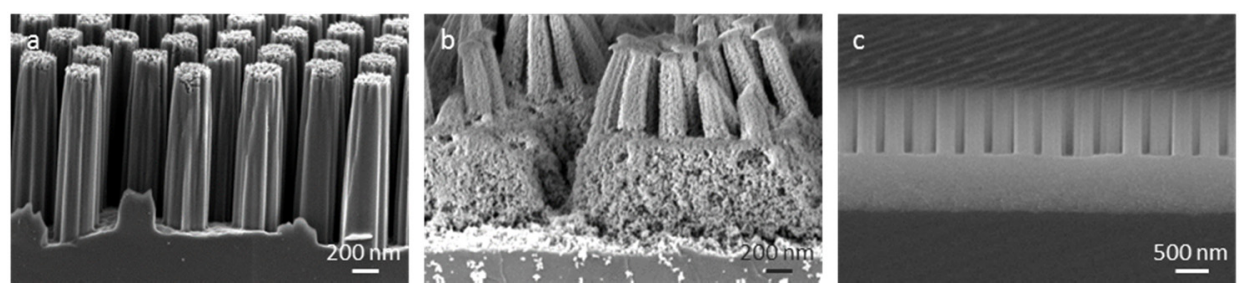

Figure 4.5. Cross-sectional HR-SEM images of Si NWs after MLCD on a) p-type, b) $n^{++}$, and c) noncleaned $\mathrm{n}^{++}$substrates.

The (projected) areal doping dose of these samples amounted to $5.9 \times 10^{15}$ atoms $/ \mathrm{cm}^{2}$ for the $\mathrm{p}$-type nanowires and $8.1 \times 10^{15}$ atoms $/ \mathrm{cm}^{2}$ for the $\mathrm{n}^{++}$nanowires, as determined by SIMS (Figure 4.6, Table 4.2). These values are again two orders of magnitude higher than the value of $4.4 \times 10^{13}$ atoms $/ \mathrm{cm}^{2}$ reported for an intrinsic planar sample. ${ }^{42}$ The source substrate contained a carborane silane, which contains 10 boron atoms per molecule, i.e., for equal coverages the doping dose is expected to be 10 times higher than for the MLD process, as has also been confirmed on flat surfaces. ${ }^{33}$ The $p$-type nanowires show an 8 times higher doping dose than the MLD samples, which is, within the experimental error, as expected. The doping dose of the $\mathrm{n}^{++}$nanowires (shown in Figure 4.5c) was even higher, even though the native oxide layer was not removed on beforehand. This means that MLCD can even be applied onto highly porous nanowires, without damaging their structure. 


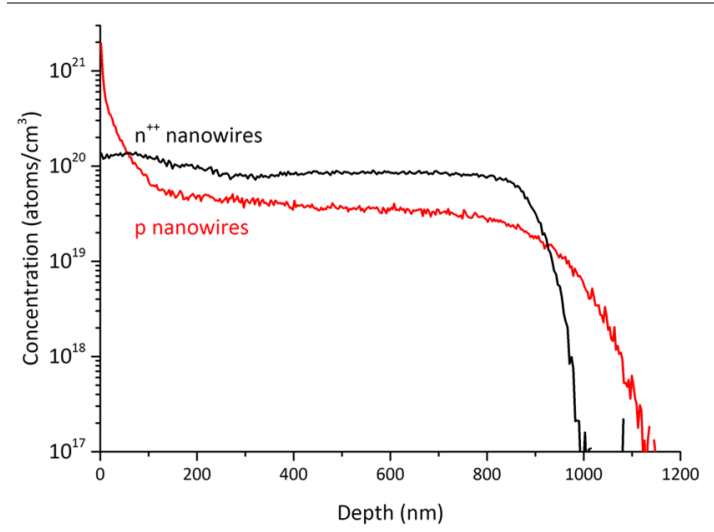

Figure 4.6. SIMS depth profiles of boron dopant atoms in $\mathrm{n}^{++}$and $\mathrm{p}$-type nanowires after MLCD.

Since the SIMS analysis does not confirm whether the dopants were electrically active, ${ }^{43,44}$ the sheet resistance of the $\mathrm{n}^{++}$nanowire arrays was measured. The sheet resistance equaled to $78 \Omega / \square$, whereas $2 \times 10^{3} \Omega / \square$ was found before for a planar intrinsic sample. ${ }^{32}$ This confirms the enhanced doping concentration of the porous nanowires. The sheet resistance can be converted into the doping peak concentration, however, the actual junction depth of these porous nanowires is unknown. When assuming a junction depth of $100 \mathrm{~nm}$, a doping peak concentration of $10^{20}$ atoms $/ \mathrm{cm}^{3}$ was obtained, which matches well with the concentrations observed in SIMS (Figure 4.6) and thus indicates that the dopants are predominantly electrically active. Since this sample showed one of the highest doping doses in the SIMS measurements, it is assumed that the implemented dopants are also electrically active in the other samples.

\subsubsection{Monolayer doping with an external capping layer}

To combine the advantages of MLD in conformal monolayer formation on the nanowires directly and of MLCD in supplying an external capping layer that avoids further wet etching steps on the target sample, a combination of MLD and MLCD was tested (Scheme 4.1c). The dopant-containing monolayer was coupled to the nanowires, but the oxide capping layer was applied as a dummy substrate. After RTA, HR-SEM images (Figure 4.7) showed that both $\mathrm{p}$ and $\mathrm{n}^{++}$nanowires were still intact. The $\mathrm{n}^{++}$nanowires showed blobs on top, which could be $\mathrm{SiO}_{2}$ residuals as judged from the SEM contrast.
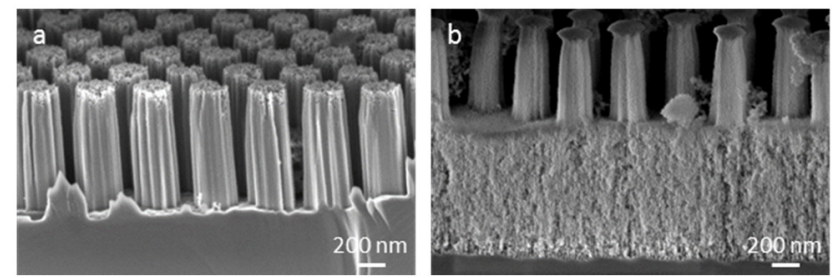

Figure 4.7. Cross-sectional HR-SEM images of Si NWs after MLD with an external capping layer on a) p-type and b) $\mathrm{n}^{++}$substrates. 
The (projected) areal doping dose was determined to be $1.9 \times 10^{15}$ atoms $/ \mathrm{cm}^{2}$ for the p-type base nanowires and $1.4 \times 10^{17}$ atoms $/ \mathrm{cm}^{2}$ for the $\mathrm{n}^{++}$nanowires (Figure 4.8 , Table 4.2). The doping dose of the $p$-type nanowires is even higher than that of the $p$-type wires doped by MLD or MLCD (when correcting for the difference in boron loading in the dopant molecules). These results indicate that the combined route can also successfully dope nanowires. The $\mathrm{n}^{++}$nanowires showed a tremendously high doping dose, which is even higher than the $\mathrm{n}^{++}$nanowires treated by MLCD. For the $\mathrm{n}^{++}$nanowires doped by the combined MLD/MLCD route, the sheet resistance was measured to be $102 \Omega / \square$ (approx. $10^{20}$ atoms $/ \mathrm{cm}^{3}$ for a junction depth of $100 \mathrm{~nm}$ ), which shows that the main part of the dopants was electrically active. These data confirm the idea that the porosity leads to a larger monolayer coverage and thus a higher doping dose. The bottleneck of the capping layer removal can be overcome in this combined MLD/MLCD process by placing a dummy substrate with an oxide layer on top of the nanowires.

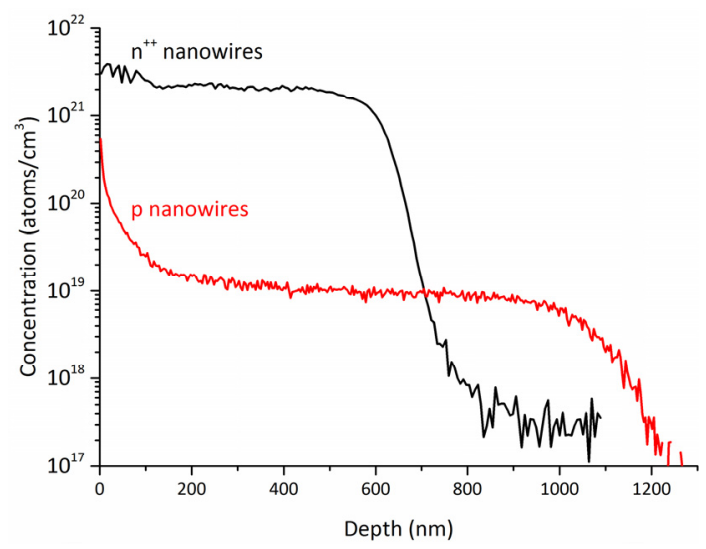

Figure 4.8. SIMS depth profiles of boron dopant atoms in $\mathrm{n}^{++}$and $\mathrm{p}$-type nanowires after MLD with an external capping layer.

\subsection{Conclusions}

Overall, high doping doses were obtained in Si NWs by making use of their porosity. MACE led to slightly porous and highly porous nanostructures, depending on the doping concentration of the base wafers. The porosity provided a higher surface area accessible for doping by monolayers of dopant-rich molecules. In the case of slightly porous ( $p$ or $n$ ) nanowires, three different monolayer doping techniques (MLD, MLCD, and a combined method) were successfully applied. This resulted in highly doped $\mathrm{p}$ - and $\mathrm{n}$-type nanowires with (projected) areal doping doses of about $10^{14}-10^{15}$ atoms $/ \mathrm{cm}^{2}$, which is much higher than for a nonporous planar reference sample $\left(10^{12}\right.$ atoms $\left./ \mathrm{cm}^{2}\right)$. Highly porous nanowires were not compatible with the capping layer used in MLD because the necessary oxide removal step after RTA damages the NWs. Two alternative techniques were found to be suitable, i.e., MLCD and MLD with an external capping layer. Especially 
the latter, combining the conformal monolayer formation of MLD with the advantage of an external capping layer of MLCD, led to an extraordinarily high doping dose of $10^{17}$ atoms $/ \mathrm{cm}^{2}$. All in all, the results show that monolayer doping techniques are perfectly suited to create arrays of highly doped Si NWs, which is beneficial for solar-tofuel devices, for example. A higher doping concentration of the base wafer leads to a higher porosity in the nanowires and thus boosts the doping dose even further. Even more control on the doping dose could be achieved by varying the annealing temperature and time.

\subsection{Acknowledgments}

Liang Ye is gratefully acknowledged for the monolayer doping of all samples, for his help, and for fruitful discussions. Arántzazu González-Campo and Rosario Núñez (Institut de Ciència de Materials de Barcelona, Spain) are gratefully acknowledged for supplying the silane-functionalized carborane compound. Wei Zhu is gratefully acknowledged for his help with the polystyrene beads monolayer formation. Roald Tiggelaar (Mesoscale Chemical Systems group, University of Twente) is kindly thanked for supplying the $\mathrm{n}^{++}$wafer. Wouter Vijselaar is gratefully acknowledged for performing the sheet resistance measurements.

\subsection{Experimental section}

\subsubsection{Materials}

Silicon wafers (<100>-oriented, $100 \mathrm{~mm}$ diameter) were obtained from Okmetic (Finland) as p-type (boron doped, resistivity 5-10 $\Omega \cdot \mathrm{cm}, \sim 10^{15}$ doping atoms $/ \mathrm{cm}^{3}$, thickness $525 \mu \mathrm{m}$, single side polished), n-type (phosphorus, $1-10 \Omega \cdot \mathrm{cm}, \sim 10^{15}$ doping atoms $/ \mathrm{cm}^{3}$, $375 \mu \mathrm{m}$, single side polished), $\mathrm{n}^{++}$(phosphorus, 0.0012-0.0015 $\Omega \cdot \mathrm{cm}$, $\sim 10^{19}$ doping atoms $/ \mathrm{cm}^{3}, 380 \mu \mathrm{m}$, double side polished), or from Topsil as an intrinsic wafer (phosphorus, $10^{4}-10^{5} \Omega \cdot \mathrm{cm}, \sim 10^{11}$ doping atoms $/ \mathrm{cm}^{3}, 515-535 \mu \mathrm{m}$, single side polished). Chemicals were used as received unless stated otherwise. Polystyrene (PS) spheres of $500 \mathrm{~nm}$ diameter functionalized with carboxyl groups were obtained from Polysciences. Allylboronic acid pinacol ester (97\%), mesitylene (>98\%), poly(methyl methacrylate) (PMMA, $M_{w}=350 \mathrm{kDa}$ ), and sodium dodecyl sulfate (SDS, >99\%) were obtained from Sigma-Aldrich. Acetone (pure) and ethanol (absolute) were purchased from VWR, anisole (99\%) from Acros, dichloromethane (99.7\%) from Actu-All and $1 \mathrm{H}, 1 \mathrm{H}, 2 \mathrm{H}, 2 \mathrm{H}$-perfluorodecyltrichlorosilane (PFDTS, 97\%) from ABCR. Hydrofluoric acid (VLSI, HF, 50\%), sulfuric acid ( $\left.\mathrm{VLSI}, \mathrm{H}_{2} \mathrm{SO}_{4}, 96 \%\right)$, hydrochloric acid (VLSI, $\mathrm{HCl}, 36 \%$ ), hydrogen peroxide (VLSIn, $\left.\mathrm{H}_{2} \mathrm{O}_{2}, 31 \%\right)$, and nitric acid (VLSI, $\mathrm{HNO}_{3}, 69 \%$ ) were obtained from BASF. Buffered hydrogen fluoride (VLSI, BOE 7:1) and $1 \%$ aqueous hydrofluoric acid (VLSI, HF) were ordered from Technic France. Toluene was obtained from a solvent purification system (MB SPS-800). A silane-functionalized carborane compound, 
1-triethoxysilyl-2-methyl-carborane, was prepared according to the literature. ${ }^{45,46} \mathrm{Milli}-\mathrm{Q}$ water with a resistivity $>18 \mathrm{M} \Omega \cdot \mathrm{cm}$ was obtained from a Milli-Q Integral water purification system (Merck Millipore). Glassware used for the monolayer reactions was dried for at least $3 \mathrm{~h}$ at $120^{\circ} \mathrm{C}$.

\subsubsection{Methods}

Fabrication of Si NWs by nanoimprint lithography. Si NWs were fabricated by combining nanolithography and MACE. Specifically, nanoimprint lithography ( $p$-type, MLD route) and nanosphere lithography (other samples) were used. For the nanoimprint lithography step, a silicon mold containing a square array of holes with $200 \mathrm{~nm}$ diameter, $200 \mathrm{~nm}$ spacing, and $300 \mathrm{~nm}$ depth was obtained from Eulitha (Switzerland). Before use, the mold was cleaned with piranha solution $\left(96 \% \mathrm{H}_{2} \mathrm{SO}_{4}\right.$ and $31 \% \mathrm{H}_{2} \mathrm{O}_{2}$ mixed at $3: 1 \mathrm{v} / \mathrm{v}$ ) for 30 min, rinsed with water and dried carefully in a stream of nitrogen. An antisticking layer of PFDTS was applied by overnight gas-phase deposition in a desiccator followed by $30 \mathrm{~min}$ baking at $120^{\circ} \mathrm{C}$. Sonication in acetone for $10 \mathrm{~min}$ was used to remove any polymerized residuals.

As a target substrate, a layer of PMMA was spin coated (6 wt\% 350 kDa PMMA in anisole, $500 \mathrm{rpm}$ for $5 \mathrm{~s}, 3000 \mathrm{rpm}$ for $30 \mathrm{~s}$ ) on a piranha-cleaned silicon substrate (p-type). Nanoimprint lithography was performed by bringing the mold and substrate into contact at 10 bar pressure using a hand-press (Specac) at $180^{\circ} \mathrm{C}$ for $5 \mathrm{~min}$. The residual layer was removed by reactive ion etching using a custom-built etching machine (10 mTorr, $20 \mathrm{sccm} \mathrm{O}, 20 \mathrm{~W}, 100 \mathrm{~s}$ ).

Fabrication of Si NWs by nanosphere lithography. For the nanosphere lithography step (Scheme 4.2), close-packed arrays of PS spheres were created at the water/air interface and transferred onto a submerged silicon substrate according to a procedure adapted from the literature. ${ }^{47,48}$ More specifically, silicon wafers $\left(p, n\right.$, or $\left.n^{++}\right)$were diced into $2 \times 2 \mathrm{~cm}^{2}$ pieces, which were rinsed with ethanol and sonicated in acetone for $10 \mathrm{~min}$ to remove the particles generated during dicing. A 0.65\% w/v solution of $500 \mathrm{~nm}$ PS spheres in 1:1 water:ethanol was sonicated for several hours to make a uniform suspension. Oxygen plasma (SPI Plasma Prep II, 40 mA, $10 \mathrm{~min}$ ) was used to turn the silicon substrates hydrophilic, as well as a glass Petri dish and a small microscope glass slide. With the glass slide in the middle of the Petri dish, water was added until the level was just above the glass slide without wetting the top of the glass slide. The PS solution $(120 \mu \mathrm{L})$ was dispersed onto the glass slide in order to spread the spheres from the glass slide to the water/air interface. The water level was increased slowly, and $50 \mu \mathrm{L}$ of an aqueous $2 \% \mathrm{w} / \mathrm{v}$ SDS solution was added as the surfactant. Opposite to the side where the PS spheres started to cluster, the glass slide was carefully removed from the solution and replaced by a silicon substrate. The silicon substrate was moved underneath the PS 
spheres, after which the water level was carefully decreased by removing all water. The rest of the solution evaporated over time, resulting in well-ordered hexagonally packed PS arrays reflecting blue light when seen under an angle. The diameter of the PS spheres deposited on a Si substrate was reduced by reactive ion etching using a custom-built etching machine (10 mTorr, $20 \mathrm{sccm} \mathrm{O}_{2}, 20 \mathrm{~W}, 2.5 \mathrm{~min}$ ).

Metal-assisted chemical etching. For the MACE process, ${ }^{13,18,41}$ a silver layer of $40 \mathrm{~nm}$ was sputtered on top of a lithography-patterned Si substrate using a custom-built sputtering system. Lift-off of the PMMA features was achieved by 60 min sonication in acetone, whereas lift-off of the PS spheres was achieved by 90 min immersion and $5 \mathrm{~min}$ sonication in dichloromethane. Exposing the substrates to an aqueous solution of $4.9 \mathrm{M}$ $\mathrm{HF} / 0.2 \mathrm{M} \mathrm{H}_{2} \mathrm{O}_{2}$ for 8 min (p-type, used for MLCD and MLD with an external capping layer) or $4 \mathrm{~min}\left(\mathrm{n}^{++}\right.$, used for MLCD and MLD with an external capping layer) resulted in silicon nanowire arrays. The samples doped by MLD were etched with slightly different solutions, namely $5.8 \mathrm{M} \mathrm{HF} / 0.1 \mathrm{M} \mathrm{H}_{2} \mathrm{O}_{2}$ for 2 min (p-type) or $4.9 \mathrm{M} \mathrm{HF} / 0.1 \mathrm{M} \mathrm{H}_{2} \mathrm{O}_{2}$ for $3 \mathrm{~min}$ (n-type). After rinsing with water, the silver layer was removed by 2 min immersion in an aqueous $\mathrm{HNO}_{3}$ solution $\left(69 \% \mathrm{HNO}_{3}\right.$ diluted with deionized water in a 1:1 ratio) followed by rinsing with water. RCA2 cleaning $\left(36 \% \mathrm{HCl}, 31 \% \mathrm{H}_{2} \mathrm{O}_{2}\right.$, and deionized water mixed at $1: 1: 5 \mathrm{v} / \mathrm{v} / \mathrm{v}$ ) for $15 \mathrm{~min}$ at $70{ }^{\circ} \mathrm{C}$ was used to remove any metal residuals. Piranha cleaning for $15 \mathrm{~min}$ was used to clean the samples before doping. The nanowire fabrication and monolayer doping were performed without storage in between to avoid deterioration of the NW surface.

Monolayer doping. MLD was performed by a procedure reported before. ${ }^{32} \mathrm{~A} 5 \% \mathrm{v} / \mathrm{v}$ solution of allylboronic acid pinacol ester in mesitylene was degassed by three freeze-pump-thaw cycles. A cleaned silicon nanowire substrate was immersed in a $1 \% \mathrm{HF}$ solution for $1 \mathrm{~min}$ to remove the native silicon oxide and to create a hydrogen-terminated silicon surface. After rinsing in Milli-Q water and drying in a nitrogen stream, the silicon substrate was immersed in the degassed solution inside a nitrogen glovebox. A roundbottom flask, equipped with a capillary as a nitrogen inlet and a reflux condenser, was heated to $180^{\circ} \mathrm{C}$ in an oil bath overnight under a low continuous nitrogen flow. Afterwards the samples were rinsed with ethanol, acetone, and Milli-Q water. Samples were sonicated in acetone for $2 \mathrm{~min}(50 \%$ power, $80 \mathrm{kHz}$ ) to remove any physisorbed material and subsequently dried in a stream of nitrogen.

Following monolayer formation, a $\mathrm{SiO}_{2}$ capping layer was sputtered onto the samples using a custom-built sputtering system. A layer thickness of $100 \mathrm{~nm}$ was used for planar substrates, whereas $300 \mathrm{~nm}$ was sputtered onto nanowire substrates to fill up the voids. Backfilling with $1 \mathrm{sccm}$ of oxygen ensured the formation of stoichiometric $\mathrm{SiO}_{2}$ layers. Annealing (Solaris 150 rapid thermal annealing system) was performed by a temperature 
ramp-up at $75^{\circ} \mathrm{C} / \mathrm{s}$ to $1000{ }^{\circ} \mathrm{C}$, holding at $1000^{\circ} \mathrm{C}$ for $6 \mathrm{~s}$, and a temperature ramp-down at $125^{\circ} \mathrm{C} / \mathrm{s}$ to $400^{\circ} \mathrm{C}$. The capping layer was stripped by immersing the substrates in an aqueous buffered HF solution for $10 \mathrm{~min}$. The samples were cleaned by a piranha solution for $15 \mathrm{~min}$, followed by immersion in a $1 \% \mathrm{HF}$ solution for $1 \mathrm{~min}$.

Monolayer contact doping. MLCD was performed as described before. ${ }^{42}$ To prepare a dopant source substrate, $100 \mathrm{~nm}$ of thermal $\mathrm{SiO}_{2}\left(1100{ }^{\circ} \mathrm{C}\right.$ for $\left.45 \mathrm{~min}\right)$ was grown on a cleaned p-type silicon substrate to avoid doping losses into the source substrate. After acetone sonication for $10 \mathrm{~min}$ and piranha cleaning for $30 \mathrm{~min}$, the silane-functionalized carborane molecule was coupled covalently to the oxide layer. A $1 \% \mathrm{v} / \mathrm{v}$ solution of the carborane silane in toluene was transported into a nitrogen glovebox, where the $\mathrm{SiO}_{2}$ substrate was immersed in the solution in a closed flask. The setup was kept in a fumehood with a balloon filled with dry nitrogen to avoid moisture in the reaction solution. After overnight reaction at room temperature, the source substrate was rinsed with ethanol, acetone, and Milli-Q water. Sonication in acetone for 10 min was used to remove any physisorbed material.

Si NWs on a Si substrate prepared by the MACE process described above were subjected to $20 \mathrm{~s}$ immersion in $1 \% \mathrm{HF}$, after which they were used as the target substrate in MLCD. The target substrate, with the source substrate on top, was annealed under the same conditions as described for the MLD process. The samples were cleaned by immersing in a piranha solution for $15 \mathrm{~min}$, followed by a $1 \%$ HF dip for $1 \mathrm{~min}$.

Monolayer doping with an external capping layer. This route starts with the covalent coupling of an allylboronic acid pinacol ester monolayer on the nanowire-coated substrate, as described above for the normal MLD technique. The capping layer was not formed on the nanowire substrate but on a dummy substrate ( $100 \mathrm{~nm}$ of thermal $\mathrm{SiO}_{2}$ on p-type silicon). This external capping layer avoids the use of HF etching on the target substrate, which is used in MLD to remove the capping layer. The annealing and cleaning were equal to the MLCD procedure described above.

\subsubsection{Equipment}

High-resolution scanning electron microscopy. HR-SEM images of cross sections of the nanowire arrays were obtained with a FEI Sirion HR-SEM with a through-the-lens detector (TLD) or a Zeiss Merlin HR-SEM system with an InLens or HE-SE2 detector, operated at typical acceleration voltages of $10-15 \mathrm{kV}$ and $1.4 \mathrm{kV}$, respectively.

Contact angle measurements. Static contact angles were measured with Milli- $Q$ water on a Krüss G10 Contact Angle Measuring Instrument equipped with a CCD camera and 
drop shape analysis software. Contact angles were measured directly after the hydrosilylation reaction and averaged over three drops.

X-ray photoelectron spectroscopy. XPS measurements were performed on a Quantera SXM setup from Physical Electronics equipped with an Al K $\alpha$ X-ray source (1486.6 eV). Take-off angles of $20^{\circ}$ and $45^{\circ}$ were used for recording signals from monolayers on a planar substrate and nanowires, respectively. Collected spectra were calibrated on the C1s peak at $284.8 \mathrm{eV}$.

Secondary ion mass spectrometry. SIMS measurements were performed by Jurgen van Berkum, Philips Innovation Service. Depth profiles were recorded using time-of-flight SIMS on an Ion-Tof TOF-SIMS IV instrument in positive mode using $0.5,1$, or $2 \mathrm{keV} \mathrm{O}_{2}{ }^{+}$ ions for sputtering. The sample with p-type nanowires doped by MLD was measured using dynamic SIMS on a Cameca IMS $6 \mathrm{f}$ system with $3 \mathrm{keV} \mathrm{O}_{2}{ }^{+}$primary ions in positive mode. For both SIMS techniques concentration calibration was based on a reference ${ }^{11} \mathrm{~B}$ implant. Depth scale calibration was based on crater depth measurements using optical profilometry.

Sheet resistance measurements. The sheet resistances of the nanowire samples were measured using a four-point probe setup from Polytec in the p-type doping mode. The complete samples were probed from the top side (Figure 4.9). The peak dopant concentrations were calculated using an exponential doping profile.

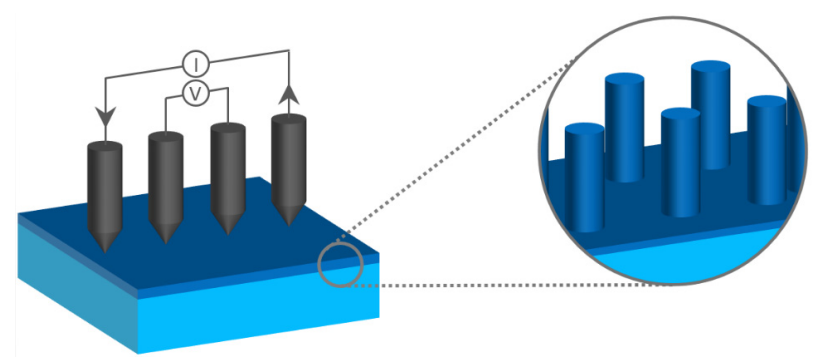

Figure 4.9. Schematic of the four-point probe setup to measure the resistivity of the nanowire samples.

\subsection{References}

1. R. Elbersen, W. Vijselaar, R.M. Tiggelaar, H. Gardeniers and J. Huskens, Adv. Mater., 2015, 27, 6781-6796.

2. G. Collins and J.D. Holmes, J. Mater. Chem., 2011, 21, 11052-11069.

3. Y.L. Wang, T.Y. Wang, P.M. Da, M. Xu, H. Wu and G.F. Zheng, Adv. Mater., 2013, 25, 51775195.

4. K.Q. Peng, X. Wang, L. Li, Y. Hu and S.T. Lee, Nano Today, 2013, 8, 75-97. 
5. N.P. Dasgupta, J.W. Sun, C. Liu, S. Brittman, S.C. Andrews, J. Lim, H.W. Gao, R.X. Yan and P.D. Yang, Adv. Mater., 2014, 26, 2137-2184.

6. N. Nafie, M.A. Lachiheb, M. Ben Rabha, W. Dimassi and M. Bouaicha, Phys. E, 2014, 56, 427430.

7. H.J. Yang, F.W. Yuan and H.Y. Tuan, Chem. Commun., 2010, 46, 6105-6107.

8. L. Yu, B. O'Donnell, M. Foldyna and P. Roca i Cabarrocas, Nanotechnology, 2012, 23, 194011.

9. J. Yoo, S.A. Dayeh, W. Tang and S.T. Picraux, Appl. Phys. Lett., 2013, 102, 093113.

10. E. Garnett and P.D. Yang, Nano Lett., 2010, 10, 1082-1087.

11. W.K. To, C.H. Tsang, H.H. Li and Z.F. Huang, Nano Lett., 2011, 11, 5252-5258.

12. Z.P. Huang, N. Geyer, P. Werner, J. de Boor and U. Gosele, Adv. Mater., 2011, 23, 285-308.

13. Z.P. Huang, H. Fang and J. Zhu, Adv. Mater., 2007, 19, 744-748.

14. X. Wang, K.Q. Peng, X.L. Wu and S.T. Lee, Electrochem. Commun., 2010, 12, 509-512.

15. J. de Boor, N. Geyer, J.V. Wittemann, U. Gosele and V. Schmidt, Nanotechnology, 2010, 21, 095302.

16. E. Koren, Y. Rosenwaks, J.E. Allen, E.R. Hemesath and L.J. Lauhon, Appl. Phys. Lett., 2009, 95, 092105.

17. Y.Q. Qu, H.L. Zhou and X.F. Duan, Nanoscale, 2011, 3, 4060-4068.

18. N. Geyer, B. Fuhrmann, Z.P. Huang, J. de Boor, H.S. Leipner and P. Werner, J. Phys. Chem. C, 2012, 116, 13446-13451.

19. C. Chiappini, X.W. Liu, J.R. Fakhoury and M. Ferrari, Adv. Funct. Mater., 2010, 20, 2231-2239.

20. X. Zhong, Y.Q. Qu, Y.C. Lin, L. Liao and X.F. Duan, ACS Appl. Mater. Interfaces, 2011, 3, 261270.

21. E.C. Garnett and P.D. Yang, J. Am. Chem. Soc., 2008, 130, 9224-9225.

22. G. Dong, F. Liu, J. Liu, H. Zhang and M. Zhu, Nanoscale Res. Lett., 2013, 8, 1-17.

23. K.Q. Peng, Y. Xu, Y. Wu, Y.J. Yan, S.T. Lee and J. Zhu, Small, 2005, 1, 1062-1067.

24. H.D. Um, J.Y. Jung, H.S. Seo, K.T. Park, S.W. Jee, S.A. Moiz and J.H. Lee, Jpn. J. Appl. Phys., 2010, 49, 04DN02.

25. J.S. Sadhu, H.X. Tian, T. Spila, J. Kim, B. Azeredo, P. Ferreira and S. Sinha, Nanotechnology, 2014, 25, 375701.

26. X. Wang, K.L. Pey, C.H. Yip, E.A. Fitzgerald and D.A. Antoniadis, J. Appl. Phys., 2010, 108, 124303.

27. H. Lee, J. Hong, S. Lee, S.D. Kim, Y.W. Kim and T. Lee, Appl. Surf. Sci., 2013, 274, 79-84.

28. K.Q. Peng, Z.P. Huang and J. Zhu, Adv. Mater., 2004, 16, 73-76.

29. S. Lee, J.H. Koo, J. Seo, S.-D. Kim, K.H. Lee, S. Im, Y.-W. Kim and T. Lee, J. Nanopart. Res., 2012, $14,1-10$.

30. J.C. Ho, R. Yerushalmi, Z.A. Jacobson, Z. Fan, R.L. Alley and A. Javey, Nat. Mater., 2008, 7, 6267.

31. J.C. Ho, R. Yerushalmi, G. Smith, P. Majhi, J. Bennett, J. Halim, V.N. Faifer and A. Javey, Nano Lett., 2009, 9, 725-730.

32. L. Ye, S.P. Pujari, H. Zuilhof, T. Kudernac, M.P. de Jong, W.G. van der Wiel and J. Huskens, ACS Appl. Mater. Interfaces, 2015, 7, 3231-3236.

33. L. Ye, A. Gonzalez-Campo, R. Nunez, M.P. de Jong, T. Kudernac, W.G. van der Wiel and J. Huskens, ACS Appl. Mater. Interfaces, 2015, 7, 27357-27361.

34. O. Hazut, A. Agarwala, I. Amit, T. Subramani, S. Zaidiner, Y. Rosenwaks and R. Yerushalmi, ACS Nano, 2012, 6, 10311-10318. 
35. O. Hazut, B.C. Huang, A. Pantzer, I. Amit, Y. Rosenwaks, A. Kohn, C.S. Chang, Y.P. Chiu and R. Yerushalmi, ACS Nano, 2014, 8, 8357-8362.

36. R.A. Puglisi, C. Garozzo, C. Bongiorno, S. Di Franco, M. Italia, G. Mannino, S. Scalese and A. La Magna, Sol. Energy Mater. Sol. Cells, 2015, 132, 118-122.

37. A.B. Sieval, R. Linke, H. Zuilhof and E.J.R. Sudholter, Adv. Mater., 2000, 12, 1457-1460.

38. J.M. Buriak, Chem. Commun., 1999, 1051-1060.

39. Y. Zhu, B. Gupta, B. Guan, S. Ciampi, P.J. Reece and J.J. Gooding, ACS Appl. Mater. Interfaces, 2013, 5, 6514-6521.

40. N.H. Maniya, S.R. Patel and Z.V.P. Murthy, Mater. Res. Bull., 2014, 57, 6-12.

41. K. Peng, M. Zhang, A. Lu, N.-B. Wong, R. Zhang and S.-T. Lee, Appl. Phys. Lett., 2007, 90, 163123.

42. L. Ye, A. González-Campo, T. Kudernac, R. Nuñez, M.P. de Jong, W.G. van der Wiel and J. Huskens, Langmuir, 2017, in press, DOI: 10.1021/acs.langmuir.7b00157.

43. V. Schmidt, J.V. Wittemann, S. Senz and U. Gosele, AdV. Mater., 2009, 21, 2681-2702.

44. K.K. Lew, L. Pan, T.E. Bogart, S.M. Dilts, E.C. Dickey, J.M. Redwing, Y.F. Wang, M. Cabassi, T.S. Mayer and S.W. Novak, Appl. Phys. Lett., 2004, 85, 3101-3103.

45. A. Gonzalez-Campo, E.J. Juarez-Perez, C. Vinas, B. Boury, R. Sillanpaa, R. Kivekas and R. Nunez, Macromolecules, 2008, 41, 8458-8466.

46. A. Gonzalez-Campo, B. Boury, F. Teixidor and R. Nunez, Chem. Mater., 2006, 18, 4344-4353.

47. G.S. Hong, C. Li and L.M. Qi, Adv. Funct. Mater., 2010, 20, 3774-3783.

48. C. Li, G. Hong, P. Wang, D. Yu and L. Qi, Chem. Mater., 2009, 21, 891-897. 


\section{Chapter 5}

\section{Maskless spatioselective functionalization of silicon nanowires}

Spatioselective functionalization of silicon nanowires was achieved without the use of a masking material. The designed process is based on a combination of metal-assisted chemical etching (MACE) to fabricate silicon nanowires and the use of hydrosilylation to form molecular monolayers. After MACE, a monolayer of 1-undecene, 1-tetradecyne, or 1,8-nonadiyne was formed on the exposed silicon nanowire surfaces. A second MACE step was expected to elongate the nanowires, so that the freshly exposed silicon surface could be functionalized using click chemistry in a subsequent functionalization step, resulting in visualization of the different segments. When monolayers of 1-undecene or 1-tetradecyne were formed on the upper segment, however, the second MACE step did not extend the nanowires. In contrast, nanowires functionalized with 1,8-nonadiyne could be elongated in the second MACE step at an approximately 8 times slower etching rate. The elongation resulted in a contrast difference in high-resolution scanning electron microscopy (HR-SEM) images, which indicated the formation of nanowires that were covered with a monolayer only at the top parts. Secondary functionalization of the monolayer with an azide-functionalized dye by click chemistry was possible, but fluorescence imaging did not allow distinction between the two segments. Instead, click chemistry with azide-functionalized nanoparticles was successfully used to functionalize the nanowires. The spatioselective presence of 1,8-nonadiyne gave a threefold higher particle density on the upper segment functionalized with 1,8-nonadiyne than on the lower segment without monolayer. These results indicate the successful spatioselective functionalization of silicon nanowires fabricated by MACE.
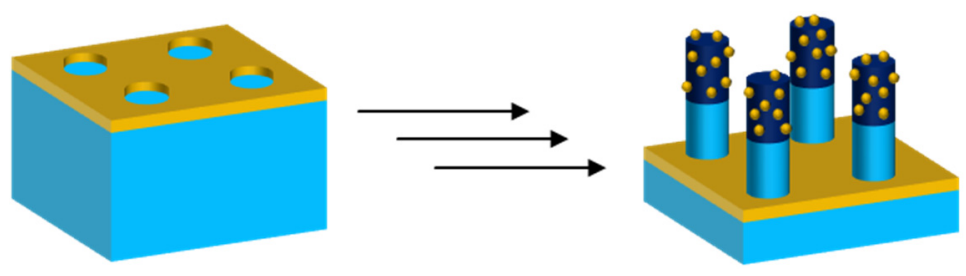


\subsection{Introduction}

Silicon nanostructures and nano/microwire arrays are attractive because of their optical, electronic, electrochemical, mechanical, and thermal properties. ${ }^{1}$ To tune these nanostructures towards a specific application, surface functionalization can offer versatility and specificity, for example, to couple photocatalysts onto the surface for hydrogen production. ${ }^{2,3}$ Whereas surface functionalization is mostly applied onto the entire surface of a $\mathrm{Si}$ micro/nanostructure, spatioselective functionalization of 3D nanostructures, i.e., the functionalization of parts of the structure, is useful when several functionalities should be combined. ${ }^{4}$ For example, oxidation and reduction reactions can be performed on two different ends of a nanowire when these are site-specifically functionalized with the respective catalysts. ${ }^{5,6}$

Spatioselective functionalization of 3D nanostructures can be achieved by prohibiting access of functionalization agents to specific areas of the structures, i.e., by protecting specific segments with a masking material, such as a polymer (resist), ${ }^{6-10}$ a metal, ${ }^{11}$ nanoparticles (NPs), ${ }^{12}$ or a membrane. ${ }^{13-15}$ These techniques, however, always require extra steps, such as the addition of the masking material, the removal of the masking material from the sites that should not be blocked and finally the mask removal after functionalization. Very few studies provide processes that avoid such multiple masking/unmasking steps. For example, Milbrat et al. have developed a method for the spatioselective functionalization of silicon microwires with axial p-n junctions. ${ }^{16}$ The charges directed by the junction, either in the dark or under illumination, were used to electrochemically deposit silver or platinum NPs in a spatially selective manner. This method, however, is applicable to metals in general but not to organic molecules. The use of molecular monolayers would offer versatility, for example, by secondary functionalization enabled by the chosen end moieties. ${ }^{17-19}$

Here, we aim for the spatioselective functionalization of silicon nanowires with molecular monolayers without the use of a masking material. We have designed a process (Scheme 5.1) based on metal-assisted chemical etching (MACE) as the nanowire fabrication method, in which a metal film (silver or gold) with patterned gaps is etched into the underlying silicon substrate upon immersion in a $\mathrm{HF} / \mathrm{H}_{2} \mathrm{O}_{2}$ solution. ${ }^{20-23}$ The diameter of the resulting nanowires is determined by the pattern in the metal film, whereas the length is determined by the etching time. Spatioselective functionalization was tested by two MACE steps with monolayer formation after the first step and functionalization of the top or bottom section of the nanowire after the second MACE step. The first MACE step is used to etch nanowires until a certain length, after which a monolayer is formed on the exposed silicon surface. A second MACE step is expected to elongate the nanowires and thus expose an unfunctionalized part of the silicon nanowires, which could then be functionalized differently in the final functionalization step. 


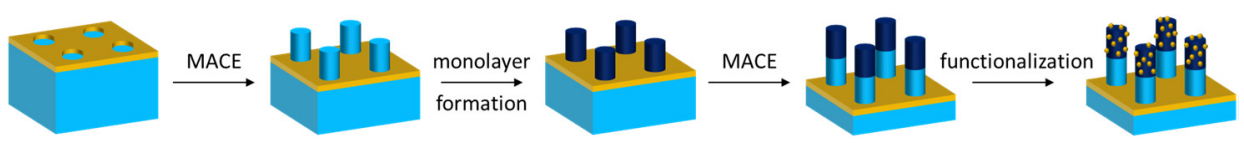

Scheme 5.1. Schematic illustration of the spatioselective functionalization process.

Monolayer formation has been achieved by hydrosilylation of alkenes or alkynes, i.e., by linking terminally unsaturated carbon-carbon bonds onto $\mathrm{H}$-terminated Si surfaces. ${ }^{24,25}$ This technique results in direct $\mathrm{Si}-\mathrm{C}$ bond formation and avoids the presence of silicon oxide in between the substrate and the monolayer. If silicon oxide would be present, the second MACE step would etch away the silicon oxide on the first part including the monolayer. Specifically, 1-undecene or 1-tetradecyne were chosen as (methylterminated) dummy molecules, and the dialkyne 1,8-nonadiyne, which can potentially be reacted further by click chemistry with azide-functionalized model catalysts, as a functional adsorbate. We here compare the different process parameters that influence the success rate of the selectively functionalized nanowire fabrication process.

\subsection{Results and discussion}

Two routes were envisaged towards the spatioselective functionalization of silicon nanowires (Scheme 5.2). Both routes are based on the same order of steps: i) MACE, ii) monolayer formation, iii) MACE, iv) functionalization. The first route (Scheme 5.2a) was designed using monolayer formation with a dummy molecule on the upper part, i.e., an alkene/alkyne (1-undecene or 1-tetradecyne, Scheme 5.3) with a nonreactive methyl end moiety. As a requirement, these monolayers should survive the second MACE step. After the second MACE step, monolayer formation with a dialkyne (1,8-nonadiyne, Scheme 5.3) could be employed, which then selectively forms on the lower part since the upper segment is already covered by a monolayer of 1-undecene or 1-tetradecyne. To visualize the difference between the two segments, the 1,8-nonadiyne monolayer could be functionalized further by copper-catalyzed click chemistry ${ }^{17}$ (Scheme 5.3) with an azidefunctionalized dye (characterized by fluorescence microscopy) or azide-functionalized gold NPs (characterized by HR-SEM). The second route (Scheme 5.2b) involves the formation of a 1,8-nonadiyne monolayer after the first MACE step. After the second MACE step to elongate the nanowires, the lower segment remains unfunctionalized and click chemistry onto the exposed alkyne moieties attached to the upper nanowire segments could be used to visualize the spatial selectivity. 


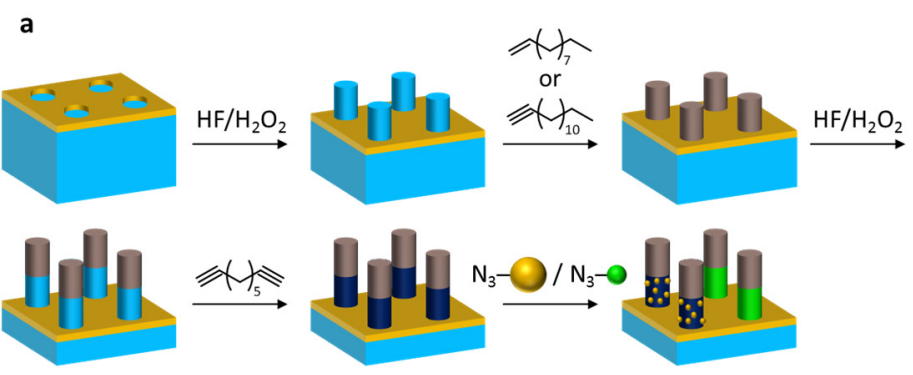

b
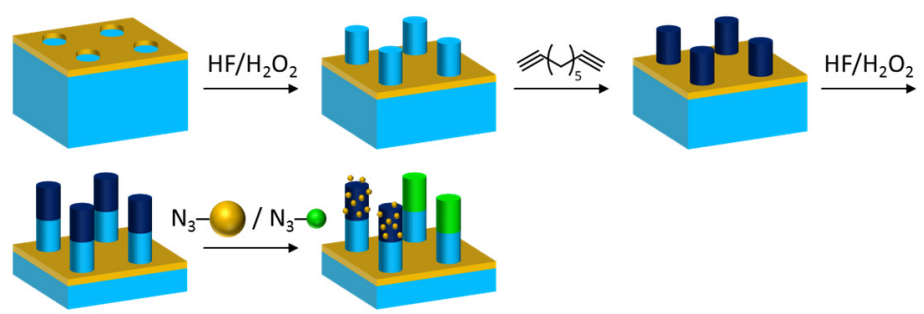

silicon

silver or gold

dummy molecule

(1-undecene or 1-tetradecyne)

1,8-nonadiyne $\quad \mathrm{N}_{3}-\mathrm{O} \equiv$ azide-Au NPs

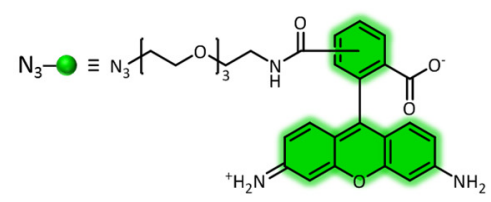

Scheme 5.2. Schematic illustration of the two different routes tested for the spatioselective functionalization of MACE-fabricated silicon nanowires: a) MACE|1-undecene/1-tetradecyne| MACE |1,8-nonadiyne |click chemistry and b) MACE|1,8-nonadiyne |MACE |click chemistry).

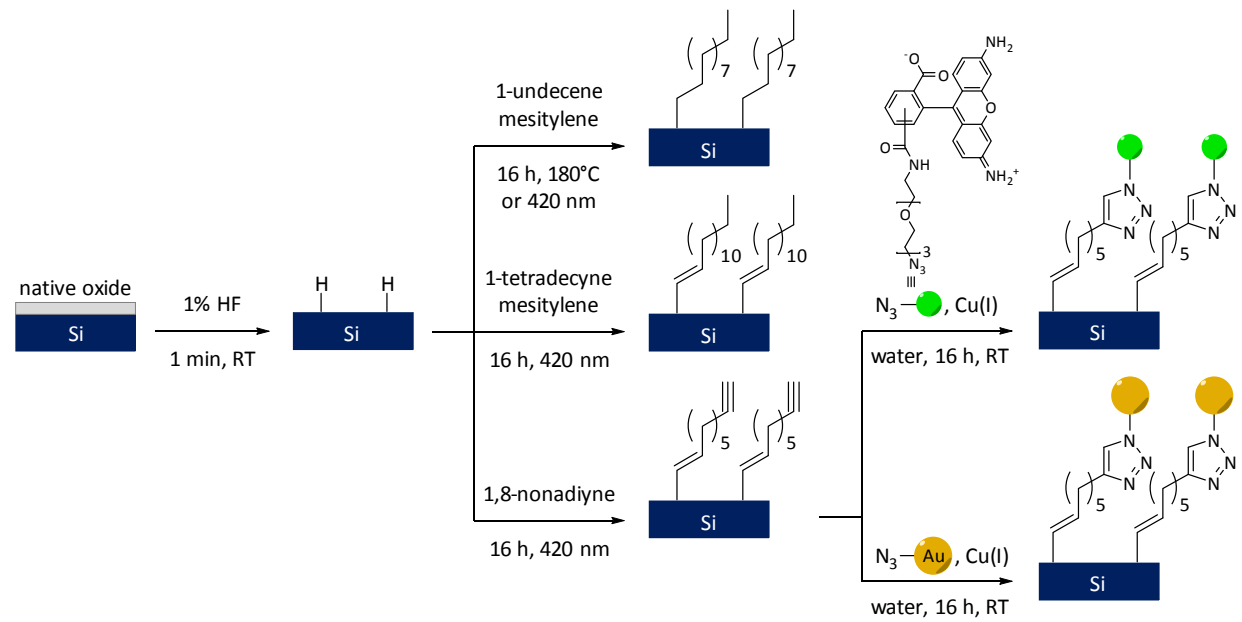

Scheme 5.3. Schematic representation of monolayer coupling to Si-H nanowires by hydrosilylation with alkenes or alkynes and subsequent click chemistry of an azide-functionalized dye or azidefunctionalized gold NPs onto the alkyne end groups of 1,8-nonadiyne. 


\subsubsection{First MACE step and monolayer formation}

Silicon nanowires were created by combining nanosphere lithography and MACE. ${ }^{26,27}$ Nanosphere lithography (Scheme 5.4) was used to form a close-packed array of polystyrene spheres on a cleaned p-type silicon substrate. The diameter of the spheres was decreased from $447 \mathrm{~nm}$ (dried state) to about $280 \mathrm{~nm}$ by oxygen reactive ion etching. After sputtering a metal film (silver or gold) on top, lift-off of the polystyrene spheres resulted in a metal film with patterned openings. Exposure of these metal-coated samples to an aqueous solution of $\mathrm{HF} / \mathrm{H}_{2} \mathrm{O}_{2}$ ensured wet etching of the silicon substrate underneath the metal layer. Immersion for $7 \mathrm{~min}(\mathrm{Ag})$ or $15 \mathrm{~min}$ (Au) resulted in nanowires with a length of about 2.1 and 1.0-1.1 $\mu \mathrm{m}$, and a diameter of 390 and $260 \mathrm{~nm}$, respectively, as shown by HR-SEM imaging (Figure 5.1a, c, e).

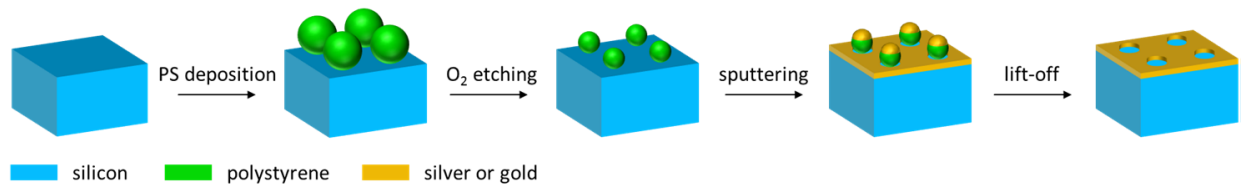

Scheme 5.4. Schematic illustration of the patterned metal film formation by nanosphere lithography.
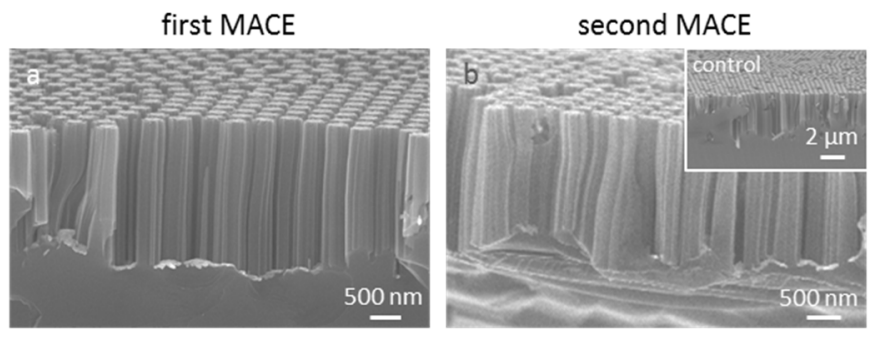

$\mathrm{Ag}$
1-undecene
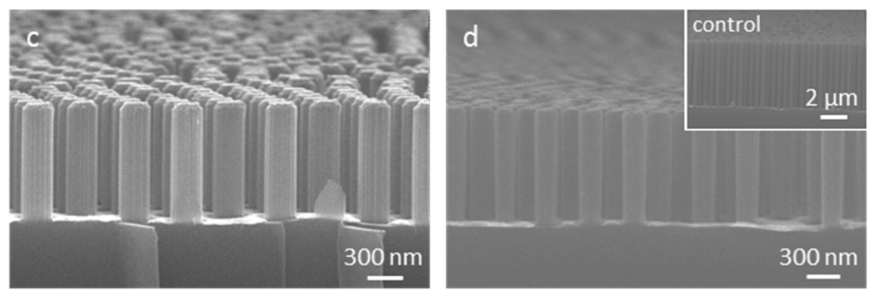

$\mathrm{Au}$

1-undecene
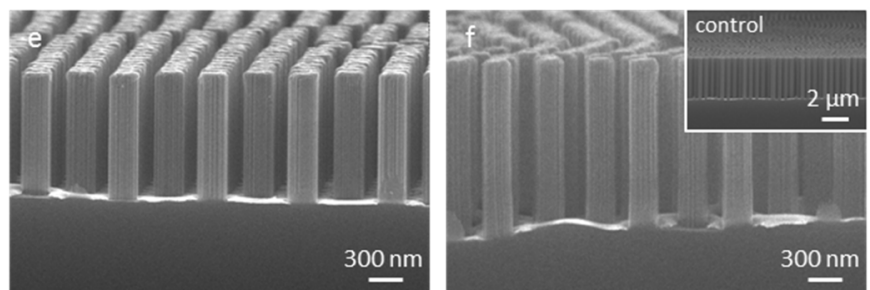

$\mathrm{Au}$

1,8-nonadiyne

Figure 5.1. Cross-sectional HR-SEM images of silicon nanowires using a-b) a Ag or c-f) a Au film, imaged $a, c, e)$ after the first MACE step and $b, d, f)$ after the second MACE step on samples with photochemically formed monolayers of a-d) 1-undecene or e,f) 1,8-nonadiyne (insets: control samples without monolayer formation). 
Monolayer formation was achieved by thermal or photochemical hydrosilylation (Scheme 5.3). ${ }^{24,25}$ This oxide-free monolayer formation technique significantly increases the chance that the monolayer stays intact during the second MACE step, since HF, present in the MACE solution, quickly removes silicon oxide. Monolayer formation was first tested by the thermal hydrosilylation method, since this generally results in higher quality monolayers than the photochemical route. The temperature used for this reaction ( $180{ }^{\circ} \mathrm{C}$ for 1 -undecene in mesitylene), however, resulted in agglomeration of the gold film (Figure 5.2). The photochemical route was therefore used by reaction under illumination of $420 \mathrm{~nm}$ light, which did not affect the metal films.
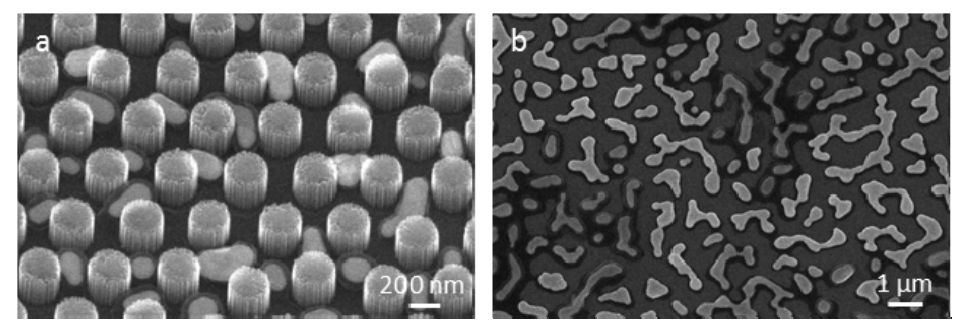

Figure 5.2. Top-view HR-SEM images of a Au film after thermal hydrosilylation $\left(180^{\circ} \mathrm{C}\right)$ imaged a) within and $b$ ) outside the nanowires region.

The photochemical hydrosilylation technique (Scheme 5.3) was used to form monolayers onto the exposed silicon nanowire surfaces after the first MACE step. On one half of the sample with nanowires, an $\mathrm{H}$-terminated silicon surface was created by short immersion in an aqueous $1 \% \mathrm{HF}$ solution. Subsequently, a monolayer of 1-undecene, 1-tetradecyne, or 1,8-nonadiyne with Si-C bonds was formed under illumination with a $420 \mathrm{~nm}$ LED. Whereas the hydrosilylation of 1-undecene and 1-tetradecyne was performed in a $5 \% \mathrm{v} / \mathrm{v}$ solution in mesitylene, 1,8-nonadiyne was used in its pure form to suppress back bending of the second alkyne group towards the hydrogen-terminated silicon surface. Cleaning was performed without ultrasonication in order to avoid damage to the metal film. The other half of the sample was used as a control, on which the second MACE step was tested without preceding monolayer formation. For the first route (Scheme 5.2a), presence of the monolayer was indicated by a contact angle of $130.7^{\circ} \pm 2.1$ (1-undecene) or $132.6^{\circ} \pm 2.2$ (1-tetradecyne) on top of the nanowires, confirming the combined effect of nanostructuring and the presence of the hydrophobic monolayer. Nanowires with a monolayer of 1,8-nonadiyne (Scheme 5.2b) were less hydrophobic due to their alkyne end moieties, as shown by a contact angle of $112.1^{\circ} \pm 3.6$. The nanowires without monolayer showed a contact angle of $40.4^{\circ} \pm 3.5,70.7^{\circ} \pm 6.5$, or $89.0^{\circ} \pm 7.2$, indicative of (varying degrees of) regrowth of native silicon oxide.

Images after the first and second MACE steps indicated a preference for using a gold film over a silver film, since i) a gold film gave more homogeneously etched samples (Figure 
5.1) and ii) the silver film was sometimes dissolved and redeposited on the lower segment of the nanowires, after which the bottom of the nanowires got etched until they broke off (data not shown). The etch rate, however, was lower for samples with a gold film compared to a silver film. This is a disadvantage since the second MACE step, and thus the exposure of the monolayer on the upper segment to the MACE solution, has to be longer.

\subsubsection{Second MACE step and monolayer formation}

Upon the second MACE step, the nanowires with a monolayer of 1-undecene (Scheme 5.2a) were unexpectedly not elongated after $15 \mathrm{~min}(\mathrm{Ag})$ or $45 \mathrm{~min}$ (Au) of MACE (Figure $5.1 b, d)$. HR-SEM images showed that the unfunctionalized nanowires increased in length to $3.7(\mathrm{Ag})$ and $4.8 \mu \mathrm{m}(\mathrm{Au})$, while the nanowires functionalized with 1-undecene remained 2.1 and $1.0 \mu \mathrm{m}$, respectively (Figure $5.1 \mathrm{~b}, \mathrm{~d}$ ). The same effect was observed for silicon nanowires functionalized with 1-tetradecyne (data not shown).

Although these data already indicated a clear effect of the presence of a monolayer on the etching behavior, contact angle measurements could not be used to verify whether the monolayers on the nanowires survived the second MACE step, since the top of the nanowires got roughened and resulted in contact angles of $130.8^{\circ} \pm 2.0$ and $138.8^{\circ} \pm 0.5$ for the functionalized (1-tetradecyne) and unfunctionalized samples, respectively. As a control, therefore, a planar substrate with a monolayer of 1-tetradecyne was immersed in the MACE solution for 10, 20, and 40 min, after which the contact angle was measured (Figure 5.3, left axis). The contact angle decreased due to exposure to the etching solution, thus indicating that the monolayer had deteriorated. These values were converted into the fraction of monolayer that was still intact (assuming a full monolayer at $\mathrm{t}=\mathrm{O}$ ) and the fraction of $\mathrm{Si}-\mathrm{H}$ formed (contact angle $78^{\circ}$ for a fully $\mathrm{H}$-terminated surface) using Cassie's law (Figure 5.3, right axis). This indicates that about $44 \%$ of the monolayer was still intact after 40 min exposure to the MACE solution, whereas a second MACE step of 45 min had not lengthened the nanowires yet. A longer second MACE step would induce even more damage to the monolayer, if it would be possible to elongate the nanowires at all. More importantly, when viewing the process envisaged in Scheme $5.2 \mathrm{a}$, the incomplete monolayer at the upper segment would be back-filled with 1,8-nonadiyne molecules in the second functionalization step. Spatioselective functionalization can thus not be achieved by this route, since 1,8-nonadiyne would be present on both segments. 


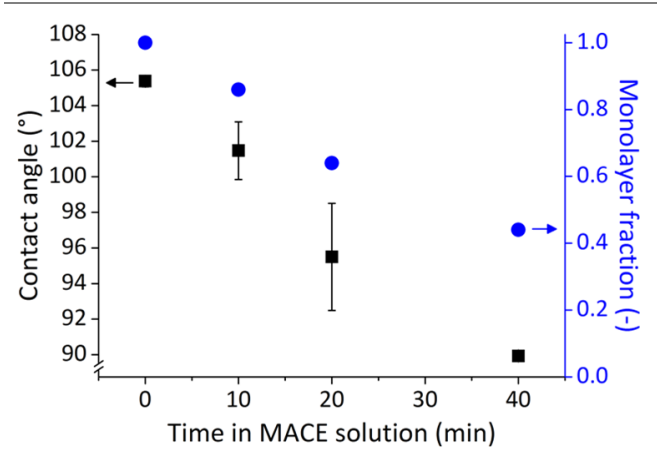

Figure 5.3. Contact angle measurements (black squares, left axis) of a 1-tetradecyne monolayer on a $p(100)$ planar sample measured after the reaction and after exposure to the MACE solution, and the monolayer fraction (blue circles, right axis) that is still intact as determined by Cassie's law.

For the other route, with 1,8-nonadiyne on the upper segment and an unfunctionalized lower segment (Scheme 5.2b), the second MACE step was tested as well. Only samples with a gold film were tested for this adsorbate for the reasons mentioned above. Additionally, it was observed that a silver film could be destroyed by contact with 1,8-nonadiyne (Figure 5.4). Contrary to the results with 1-undecene/1-tetradecyne, a second MACE step of 30 min after 1,8-nonadiyne attachment resulted in an increase of the length of the nanowires of 200-300 nm, from $1.1 \mu \mathrm{m}$ to 1.3-1.4 $\mu \mathrm{m}$ (Figure 5.1f). The unfunctionalized control samples were elongated up to $3.0 \mu \mathrm{m}$, which shows that the presence of the monolayer has a retarding effect (of approximately a factor 8 ) on the second MACE step. The effect of 30 min exposure of the 1,8-nonadiyne monolayer to the MACE solution could not be tested by contact angle measurements as described above for 1-tetradecyne (Figure 5.3), since the contact angle of a full monolayer of 1,8-nonadiyne on a planar sample $\left(82^{\circ}\right)$ was too close to that of a deteriorated monolayer $\left(\mathrm{Si}-\mathrm{H}, 78^{\circ}\right)$. This made a proper determination of the fraction of intact monolayer impossible.

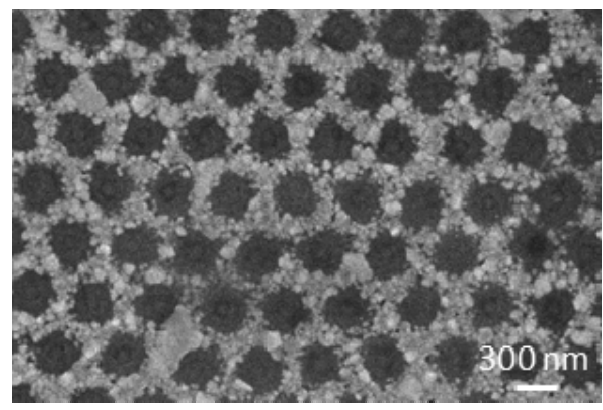

Figure 5.4. Top-view HR-SEM image of a Ag film after overnight immersion in 1,8-nonadiyne. 
The HR-SEM data do not clarify what causes the retardation or complete inhibition of the second MACE step. The difference in etching between the 1,8-nonadiyne and 1-undecene/1-tetradecyne samples seems to originate from the monolayer formation step, since the unfunctionalized control samples were etched as expected. The main differences between the two routes (Scheme 5.2) include the adsorbates (1,8-nonadiyne versus 1-undecene/1-tetradecyne) and the solvents (none versus mesitylene). Two main explanations are hypothesized: i) the MACE solution is not able to enter the voids when a hydrophobic monolayer is present and ii) a contamination at the metal/silicon interface is preventing the second MACE step. To the first point, the more hydrophilic the nanowires are, the higher the chance that the aqueous MACE solution enters the voids to continue etching. As shown by contact angle measurements, the monolayers of 1-undecene and 1-tetradecyne were more hydrophobic than those of 1,8-nonadiyne and could thus prevent the MACE solution from reaching the metal film at the bottom of the voids. To the second point, if something would be present on the metal/silicon interface, the second MACE step could be delayed or prohibited. A layer on top of the metal film, instead, does not prohibit MACE, 22 i.e., only the interface between the metal film and the underlying silicon substrate is of importance. After the first MACE step, porous silicon is formed underneath the metal film, ${ }^{22}$ which could theoretically be filled up with reagents or solvents (from the reaction or cleaning steps). This would, however, not explain why the samples with 1,8-nonadiyne could be etched further in the second MACE step. Control experiments were performed by overnight immersion of silicon samples with a gold-patterned film after lift-off, i.e., before the first MACE step, in mesitylene, 1-undecene, or 1,8-nonadiyne. The first MACE step was successful for all of these samples, although the etch rate was lower than for samples without immersion. Preliminary tests on immersion of silicon nanowires in mesitylene and a subsequent second MACE step, did not conclusively show whether the nanowires could be elongated or not. Further research is thus required to verify whether these hypotheses are correct.

\subsubsection{Secondary functionalization}

In the second route (Scheme 5.2b), the copper-catalyzed click reaction ${ }^{17,28}$ was used to investigate whether the 1,8-nonadiyne monolayer was still present after the second MACE step and spatioselective functionalization could be achieved. In this reaction, an azide-functionalized molecule or particle is coupled onto the alkyne end moiety of the 1,8-nonadiyne monolayer using $\mathrm{Cu}(\mathrm{I})$ as the catalyst (Scheme 5.3). Either an azidefunctionalized dye was chosen for detection by fluorescence microscopy, or azidefunctionalized gold NPs to allow detection by high-resolution scanning electron microscopy. A Cu(I) stabilizing ligand (TBTA) was used to increase the rate of the click chemistry within the voids between the nanowires. ${ }^{29}$ 
After functionalization with azide-fluor 488, the nanowires were scraped off the surface and immobilized onto a microscope glass slide. As a disadvantage, this induced a high background fluorescence and showed irregularly shaped fluorescent objects, probably due to residuals of the gold film used for MACE. Nonetheless, fluorescence microscopy showed needle-shaped objects that are most likely the functionalized nanowires based on their size (Figure 5.5). Comparison of the length of the wires in the fluorescence and brightfield images indicates, however, that the resolution of these images is too low to conclude whether the $200-300 \mathrm{~nm}$ bottom segments had remained unfunctionalized. Confocal microscopy did not solve this resolution issue. A longer second MACE step would be needed to increase the contrast between the two segments but could also damage the 1,8-nonadiyne monolayer on the upper segment and thus was not pursued further here.

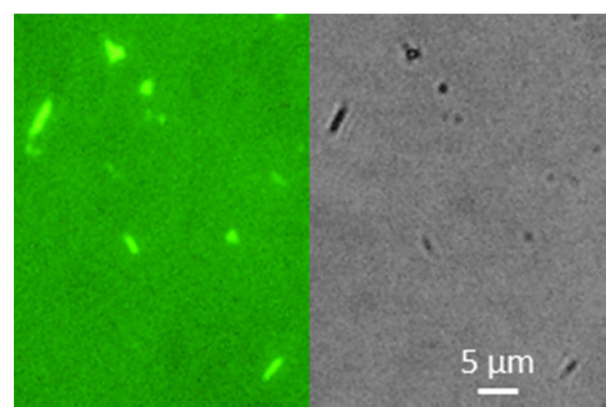

Figure 5.5. Microscopy images after click chemistry with an azide-functionalized dye onto silicon nanowires (Au film) functionalized with a monolayer of 1,8-nonadiyne on the upper segment (left: fluorescence microscopy image, 200 ms exposure time, brightness increased by 30\%, right: brightfield image, 5 ms exposure time).

To circumvent the practical issues associated with fluorescent dye functionalization and imaging, click chemistry with azide-functionalized gold NPs (10 nm diameter) was tested. In this way, the nanowires did not have to be transferred to a microscope slide, since HR-SEM imaging of sample cross sections was sufficient, and a higher resolution could be obtained. After click chemistry, HR-SEM images showed a clear contrast between the upper functionalized segment and the lower unfunctionalized segment (Figure 5.6a). This contrast was also visible when imaging before click chemistry (Figure 5.1f) but was more clearly visible in the higher resolution image of Figure 5.6a. Since the contrast was not visible on the control samples, it is assumed that this indicates the presence of a 1,8-nonadiyne monolayer on the upper segment. A carbon-containing layer is known to give a darker contrast in SEM images compared to the, most likely, silicon oxidecontaining lower segment which is much brighter. The height of the lower segment in the HR-SEM image (Figure 5.6a) corresponds well with the nanowire length increase observed for the second MACE step, as discussed above. 

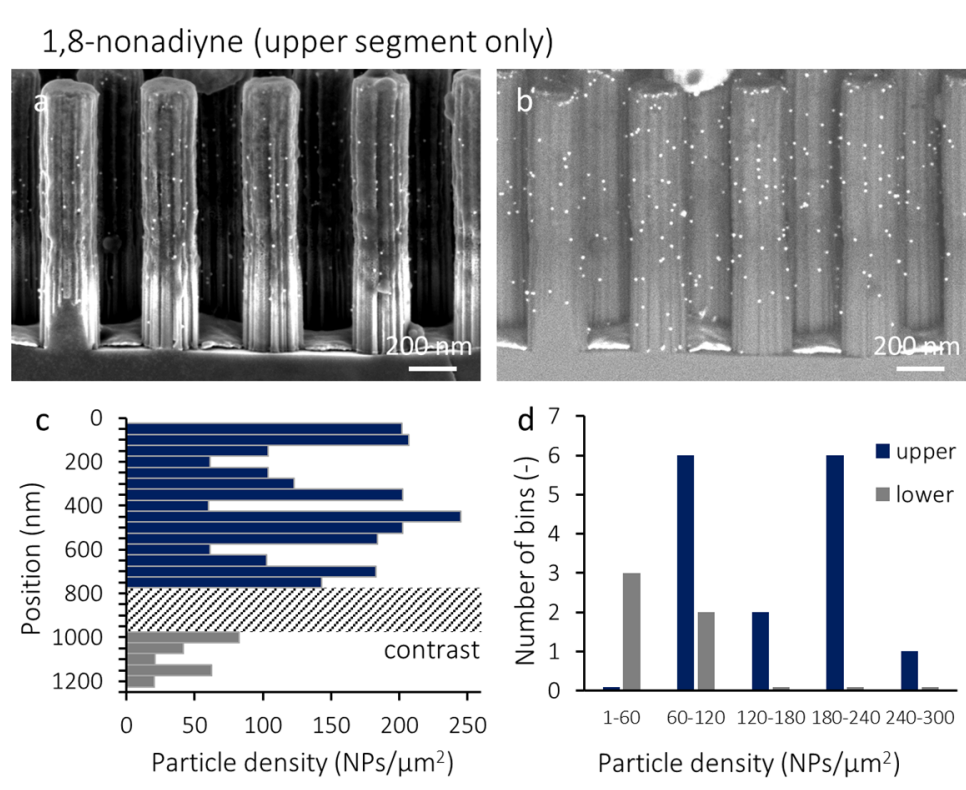

1-undecene
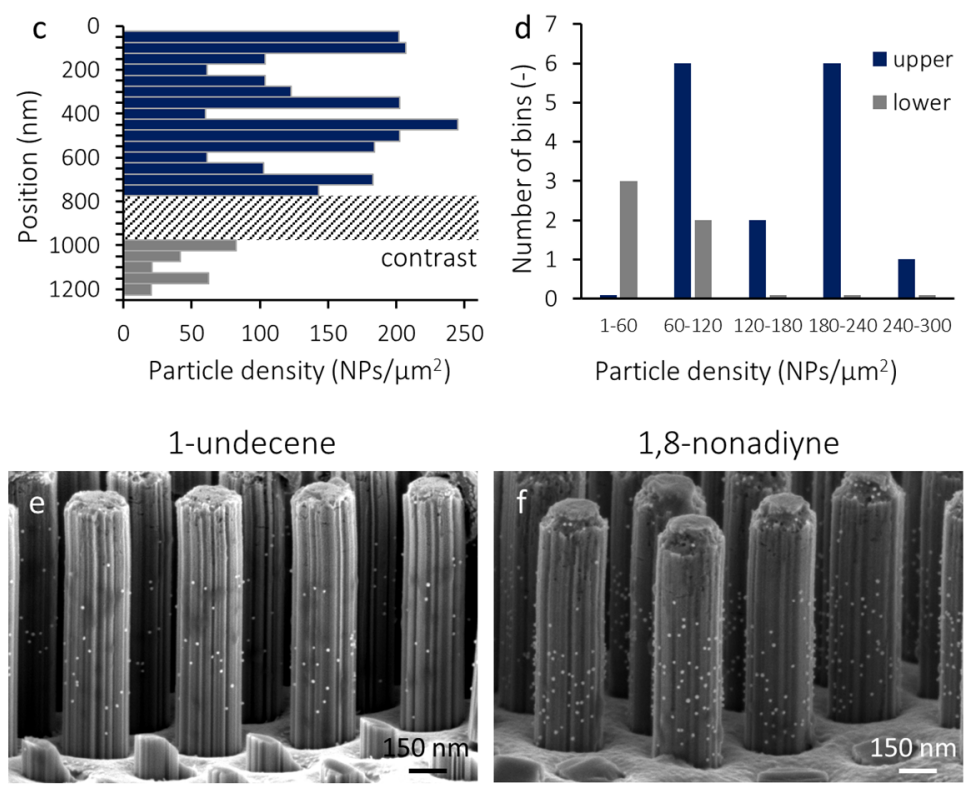

Figure 5.6. a,b) Cross-sectional HR-SEM images of silicon nanowires (Au film) after click chemistry with azide-functionalized gold NPs on a) nanowires functionalized with a 1,8-nonadiyne monolayer on the upper segment and b) the corresponding ESB image. c) The particle count along the nanowire length (bin size $50 \mathrm{~nm}$ ). d) The number of bins from (c) sorted on particle density for the two segments. e,f) Cross-sectional HR-SEM images after click chemistry with azide-functionalized gold NPs on nanowires fully functionalized with a monolayer of e) 1-undecene and f) 1,8-nonadiyne.

After click chemistry, the azide-functionalized gold NPs should be present on the upper segment only. An energy-selective backscatter (ESB) detector was used to reflect compositional variations on the sample based on atomic number, which showed NPs over the entire nanowire length (Figure 5.6b). Nonetheless, particle counting along the nanowire length (Figure 5.6c) clearly showed more NPs on the segment above the contrasting line. Quantitatively, the average particle densities, as calculated from the SEM images, were $136 \pm 70 \mathrm{NPs} / \mu^{2}$ on the 1,8-nonadiyne functionalized upper segment $(0-0.8 \mu \mathrm{m})$ and $45 \pm 27 \mathrm{NPs} / \mu \mathrm{m}^{2}$ on the unfunctionalized lower segment (1.0-1.3 $\left.\mu \mathrm{m}\right)$, where the gradual transition in contrast between 0.8 and $1.0 \mu \mathrm{m}$ was not taken into account. The threefold difference between the two segments ( $p<0.01$, Student's $t$-test) 
is also reflected in Figure 5.6d, where the upper segment shows higher particle densities than the lower segment. Nonspecific adsorption of the gold NPs was also observed on control samples with a monolayer of 1-undecene on the full nanowires (Figure 5.6e, $81 \pm 15 \mathrm{NPs} / \mu^{2}$ ), but the average particle density was much lower than on the same nanowires with 1,8-nonadiyne (Figure 5.6f, $212 \pm 17 \mathrm{NPs} / \mu \mathrm{m}^{2}$ ). Although the NPs were inhomogeneously distributed along the nanowires that were fully functionalized with 1,8-nonadiyne, particles were clearly observed at the lower part of these nanowires. The much lower amount of particles present at the lower part of the nanowires shown in Figure $5.6 \mathrm{~b}$ should thus be due to the absence of a 1,8-nonadiyne monolayer. Overall, on nanowires fabricated by the 1,8-nonadiyne route (Scheme $5.2 \mathrm{~b}$ ) the differences in contrast and in the amount of NPs present on the two segments clearly indicate successful spatioselective functionalization.

\subsection{Conclusions and outlook}

In summary, the spatioselective functionalization of silicon nanowires was tested using consecutively: i) MACE, ii) monolayer formation, iii) MACE, iv) functionalization by click chemistry. Nanowires were successfully fabricated and subsequently functionalized with 1-alkenes/alkynes by hydrosilylation. When the top segment, exposed after the first MACE step, was covered with a monolayer of 1-undecene or 1-tetradecyne, the second MACE step inexplicably did not elongate the nanowires. In contrast, nanowires functionalized with 1,8-nonadiyne could be lengthened from $1.1 \mu \mathrm{m}$ to $1.3-1.4 \mu \mathrm{m}$. A contrast difference observed in HR-SEM images of the 1,8-nonadiyne-functionalized nanowires indicated the formation of the 1,8-nonadiyne monolayer only on the top section of the nanowires. Click chemistry with an azide-functionalized dye was possible, but presence of the dye only on the top section could not be verified. The reaction with azide-functionalized nanoparticles, however, confirmed the successful secondary functionalization and showed a higher particle density on the upper segment. These results indicate that spatioselective functionalization of silicon nanowires by MACE is possible.

Further research could elucidate why/when the second MACE step does (not) work. To conclude whether the hydrophobicity of the monolayer is the origin, testing the second MACE step on nanowires functionalized with a hydrophilic alkyne, for example with a carboxylic acid end group, ${ }^{30,31}$ could be useful. Alternatively, it might help to add a surfactant, e.g., ethanol ${ }^{32}$ or sodium dodecyl sulfate, to lower the interfacial tension. Next, the influence of the metal/silicon interface could be studied further, i.e., whether something present at this interface is prohibiting the second MACE step. For example, the role of mesitylene as a solvent can be eliminated by monolayer formation in pure 1-undecene/1-tetradecyne or in a solution of 1,8-nonadiyne in mesitylene. Considering the specific click chemistry reaction used, the nonspecific adsorption of the azide- 
functionalized gold NPs on the segment without 1,8-nonadiyne should be lowered, for example using a poly(ethylene glycol) antifouling monolayer.

The spatioselective formation of a 1,8-nonadiyne monolayer was shown here by a contrast difference in the HR-SEM images, and azide-functionalized nanoparticles were successfully used to functionalize the nanowires further. The compounds used here, however, are just an example for the applicability of this process. End group of the alkynecontaining molecules used in the monolayer formation can be chosen as desired, and the subsequent secondary functionalization can be tuned accordingly. The fabrication method also offers versatility, since i) the height of each segment could be tuned, depending on the application, for instance different amounts of catalysts based on their respective catalytic activities, ii) more than two segments could be made by adding more MACE/functionalization cycles. Thus, this fabrication method offers a relatively simple solution for the creation of spatioselectively functionalized nanowires without the use of a masking material.

\subsection{Acknowledgments}

Carlo Nicosia is gratefully acknowledged for the synthesis of TBTA. Wei Zhu is gratefully acknowledged for his help with the polystyrene beads monolayer formation.

\subsection{Experimental section}

\subsubsection{Materials}

Silicon wafers (<100>-oriented, $100 \mathrm{~mm}$ diameter, single side polished) were obtained from Okmetic (Finland) as p-type (boron, resistivity 5-10 $\Omega \cdot \mathrm{cm}$, thickness $525 \mu \mathrm{m}$ ). Polystyrene spheres of $500 \mathrm{~nm}$ diameter functionalized with carboxyl groups were obtained from Polysciences. Azide-functionalized gold NPs of $10 \mathrm{~nm}$ diameter were obtained from NanoCS, with a particle concentration of $0.5 \mathrm{mg} / \mathrm{mL}$ in water (based on gold salt, $2.8 \times 10^{13}$ particles $/ \mathrm{mL}$ ), a size distribution $<15 \%$ and a poly(ethylene glycol) linker between the NPs and the azide groups. Mesitylene (>98\%, Sigma-Aldrich) and 1,8-nonadiyne (98\%, Sigma-Aldrich) were dried over molecular sieves $(0.3 \mathrm{~nm})$. Dichloromethane (99.7\%, Actu-All) was dried over anhydrous magnesium sulphate (Merck). Acetone (pure, VWR), L-ascorbic acid (>99\%, Sigma-Aldrich), azide-fluor 488 (>90\%, Sigma-Aldrich), copper(II) sulfate pentahydrate $(99.995 \%$ metals basis, $\mathrm{Cu}(\mathrm{II}) \mathrm{SO}_{4} .5 \mathrm{H}_{2} \mathrm{O}$, Sigma-Aldrich), ethanol (absolute, VWR), hydrofluoric acid $1 \%$ (aqueous, VLSI, 1\% HF, Technic France), hydrofluoric acid 50\% (aqueous, VLSI, 50\% HF, BASF), hydrogen peroxide (VLSIn, $\mathrm{H}_{2} \mathrm{O}_{2}, 31 \%$, BASF), sodium dodecyl sulfate (SDS, >99\%, SigmaAldrich), 1-tetradecyne (>97\%, Sigma-Aldrich), and 1-undecene (97\%, Sigma-Aldrich) were used as received. Tris-(benzyltriazolylmethyl)amine (TBTA) was synthesized according to a procedure from the literature. ${ }^{33}$ Hexane was obtained from a solvent 
purification system (MB SPS-800). Milli-Q water with a resistivity $>18 \mathrm{M} \Omega \cdot \mathrm{cm}$ was obtained from a Milli-Q Integral water purification system (Merck Millipore). Glassware used for the hydrosilylation reactions was dried overnight at $120^{\circ} \mathrm{C}$.

\subsubsection{Methods}

Fabrication of silicon nanowires. Silicon nanowires were fabricated by combining nanosphere lithography (Scheme 5.4) and MACE. A close-packed array of $500 \mathrm{~nm}$ polystyrene (PS) spheres was formed at the water/air interface and transferred onto a silicon substrate according to a procedure adapted from the literature. ${ }^{34,35}$ Specifically, small $2 \times 2 \mathrm{~cm}^{2}$ samples were diced from silicon wafers ( $p$-type), which were rinsed with ethanol and sonicated in acetone for $10 \mathrm{~min}$ to remove particles generated during dicing. A $0.65 \% \mathrm{w} / \mathrm{v}$ solution of $500 \mathrm{~nm}$ PS spheres in 1:1 water:ethanol was sonicated for several hours to make a uniform suspension. The silicon samples, a glass Petri dish, and a small microscope glass slide were turned hydrophilic by oxygen plasma (SPI Plasma Prep II, $40 \mathrm{~mA}, 10 \mathrm{~min}$ ). The glass slide was put in the middle of the Petri dish, after which water was added until the level was just above the glass slide without wetting the top of the glass slide. The PS solution $(120 \mu \mathrm{L})$ was dispersed onto the glass slide in order to diffuse the spheres from the glass slide to the water/air interface. After slowly increasing the water level, $50 \mu \mathrm{L}$ of an aqueous $2 \% \mathrm{w} / \mathrm{v}$ SDS solution was added as surfactant. Opposite to the side where the PS spheres started to cluster, the glass slide was carefully removed from the solution, and a silicon substrate was submerged. The silicon substrate was moved underneath the PS spheres, and the water level was carefully lowered by removing all water. The rest of the solution evaporated over time, resulting in wellordered hexagonally packed PS arrays reflecting blue light when seen under an angle.

The diameter of the PS spheres was reduced by reactive ion etching using a custom-built etching machine (10 mTorr, $20 \mathrm{sccm} \mathrm{O}, 20 \mathrm{~W}, 2.5 \mathrm{~min}$ ). Then, a silver layer of $40 \mathrm{~nm}$ (100 W, $1.0 \mathrm{~min}$ ) or a gold layer of $30 \mathrm{~nm}(200 \mathrm{~W}, 45 \mathrm{~s})$ was sputtered on top using a custom-built sputtering system. Lift-off of the PS spheres was achieved by $90 \mathrm{~min}$ immersion and $5 \mathrm{~min}$ sonication in dichloromethane. For the MACE step, ${ }^{22,26,27}$ the substrates were immersed in an aqueous solution of $5.8 \mathrm{M} \mathrm{HF/0.1} \mathrm{M} \mathrm{H} \mathrm{H}_{2}(50 \% \mathrm{HF}$, $31 \% \mathrm{H}_{2} \mathrm{O}_{2}$, and water mixed at 20/0.9/79.1 v/v\%) for $7 \mathrm{~min}(\mathrm{Ag})$ or $15 \mathrm{~min}(\mathrm{Au})$ to obtain silicon nanowire arrays. The samples were rinsed with water and dried under nitrogen. The samples were cut into two halves to use one half as a control without monolayer formation. After monolayer formation on the other half, the second MACE step was performed on both halves in the same MACE solution for $15 \mathrm{~min}(\mathrm{Ag})$ and 30 or $45 \mathrm{~min}$ (Au).

Monolayer formation. Monolayers of 1-undecene, 1-tetradecyne, or 1,8-nonadiyne were formed by hydrosilylation (Scheme 5.3). The adsorbate solution (3-6 mL), consisting of a 
$5 \% \mathrm{v} / \mathrm{v}$ solution of 1 -undecene in mesitylene, a $5 \% \mathrm{v} / \mathrm{v}$ solution of 1 -tetradecyne in mesitylene or pure 1,8-nonadiyne, was degassed by four freeze-pump-thaw cycles for the hydrosilylation reaction. After MACE and HR-SEM imaging, a hydrogen-terminated surface was reactivated on the p-type silicon nanowires by 1 min immersion in an aqueous $1 \% \mathrm{HF}$ solution, to remove the native oxide grown during HR-SEM imaging. The substrates were then immediately immersed in the degassed adsorbate solution inside a nitrogen glovebox. The reaction flask was equipped with a capillary as a nitrogen inlet and a reflux condenser. The hydrosilylation reactions were performed overnight under continuous nitrogen flow at $180{ }^{\circ} \mathrm{C}$ (for 1-undecene) for the thermal route and under illumination of a $420 \mathrm{~nm}$ LED $(130 \mathrm{~mW})$ at a $1 \mathrm{~cm}$ distance for the photochemical route (for 1-undecene, 1-tetradecyne, or 1,8-nonadiyne). The nanowires were cleaned by immersion in hexane, rinsed with ethanol and dichloromethane, and subsequently dried in a stream of nitrogen. The samples were not sonicated in order to keep the metal film intact.

Click chemistry. Copper-catalyzed azide-alkyne cycloaddition (click chemistry, Scheme 5.3) was used to couple the fluorescent dye azide-fluor 488 or azide-functionalized gold NPs onto a 1,8-nonadiyne monolayer. Silicon nanowires were overnight incubated with $60 \mu \mathrm{L}$ of the azide solution ( $2 \mathrm{mM}$ azide-fluor 488 in water, or azide-functionalized gold NPs as received) and $60 \mu \mathrm{L}$ of the catalyst solution ( $1.5 \mathrm{mM} \mathrm{Cu}(\mathrm{II}) \mathrm{SO}_{4} .5 \mathrm{H}_{2} \mathrm{O}, 1.5 \mathrm{mM}$ TBTA, $60 \mathrm{mM} \mathrm{L}$-ascorbic acid in ethanol/water, ratio $5: 2 \mathrm{v} / \mathrm{v}$ ) in a silicone isolator (Electron Microscopy Sciences). A glass slide on top was used to avoid solvent evaporation. Afterwards, the samples were sequentially rinsed with water, ethanol, water, ethanol, immersed in acetone to remove the glue of the isolator, sonicated in ethanol for $1 \mathrm{~min}$ and dried in a stream of nitrogen.

\subsubsection{Equipment}

Contact angle measurements. Static contact angles were measured with Milli-Q water on a Krüss G10 Contact Angle Measuring Instrument equipped with a CCD camera and drop shape analysis software. Contact angles were measured directly after the hydrosilylation reaction and averaged over three drops.

High-resolution scanning electron microscopy. HR-SEM images of cross sections of the nanowire arrays were obtained with a FEI Sirion HR-SEM with a through-the-lens detector (TLD) or a Zeiss Merlin HR-SEM system with an InLens or ESB detector, operated at typical acceleration voltages of $10-15 \mathrm{kV}$ and $1.6 \mathrm{kV}$, respectively. Fresh cuts were made to assess the cross sections after each step.

Fluorescence microscopy. Silicon nanowires functionalized with azide-functionalized dye were removed from the surface using a scalpel and immersed in a small volume of 
ethanol. The solution was pipetted onto a microscope glass slide, after which the ethanol was evaporated to confine the nanowires onto the glass slide. Fluorescence microscopy images were acquired in air on an Olympus inverted research microscope IX71 equipped with a mercury burner U-RFL-T as light source and a digital Olympus DP70 camera. Blue excitation ( $\lambda_{e x}=490-510 \mathrm{~nm}$ ) and green emission $\left(\lambda_{e m}=520-550 \mathrm{~nm}\right)$ were filtered using a Chroma filter cube.

\subsection{References}

1. N.P. Dasgupta, J.W. Sun, C. Liu, S. Brittman, S.C. Andrews, J. Lim, H.W. Gao, R.X. Yan and P.D. Yang, Adv. Mater., 2014, 26, 2137-2184.

2. I. Oh, J. Kye and S. Hwang, Nano Lett., 2012, 12, 298-302.

3. S.Y. Reece, J.A. Hamel, K. Sung, T.D. Jarvi, A.J. Esswein, J.J.H. Pijpers and D.G. Nocera, Science, 2011, 334, 645-648.

4. A. Walther and A.H.E. Müller, Chem. Rev., 2013, 113, 5194-5261.

5. Y.Q. Qu, L. Liao, R. Cheng, Y. Wang, Y.C. Lin, Y. Huang and X.F. Duan, Nano Lett., 2010, 10, 1941-1949.

6. Y.Q. Qu, T. Xue, X. Zhong, Y.C. Lin, L. Liao, J.N. Choi and X.F. Duan, Adv. Funct. Mater., 2010, 20, 3005-3011.

7. T.E. McKnight, C. Peeraphatdit, S.W. Jones, J.D. Fowlkes, B.L. Fletcher, K.L. Klein, A.V. Melechko, M.J. Doktycz and M.L. Simpson, Chem. Mater., 2006, 18, 3203-3211.

8. X.Y. Ling, I.Y. Phang, C. Acikgoz, M.D. Yilmaz, M.A. Hempenius, G.J. Vancso and J. Huskens, Angew. Chem., Int. Ed., 2009, 48, 7677-7682.

9. I. Park, Z.Y. Li, A.P. Pisano and R.S. Williams, Nano Lett., 2007, 7, 3106-3111.

10. C. Liu, J.Y. Tang, H.M. Chen, B. Liu and P.D. Yang, Nano Lett., 2013, 13, 2989-2992.

11. J.S. Yu, J.Y. Kim, S. Lee, J.K.N. Mbindyo, B.R. Martin and T.E. Mallouk, Chem. Commun., 2000, 2445-2446.

12. A.M. Yake, C.E. Snyder and D. Velegol, Langmuir, 2007, 23, 9069-9075.

13. Z. Wang and M. Brust, Nanoscale Res. Lett., 2007, 2, 34-39.

14. C.T. Sousa, D.C. Leitao, J. Ventura, P.B. Tavares and J.P. Araujo, Nanoscale Res. Lett., 2012, 7, 168.

15. K. Skinner, C. Dwyer and S. Washburn, Nano Lett., 2006, 6, 2758-2762.

16. A. Milbrat, R. Elbersen, R. Kas, R.M. Tiggelaar, H. Gardeniers, G. Mul and J. Huskens, Adv. Mater., 2016, 28, 1400-1405.

17. Y. Li and C.Z. Cai, Chem. - Asian J., 2011, 6, 2592-2605.

18. N. Herzer, C. Haensch, S. Hoeppener and U.S. Schubert, Langmuir, 2010, 26, 8358-8365.

19. M.D. Yilmaz and J. Huskens, Soft Matter, 2012, 8, 11768-11780.

20. Z.P. Huang, N. Geyer, P. Werner, J. de Boor and U. Gosele, Adv. Mater., 2011, 23, 285-308.

21. Y.Q. Qu, H.L. Zhou and X.F. Duan, Nanoscale, 2011, 3, 4060-4068.

22. N. Geyer, B. Fuhrmann, Z.P. Huang, J. de Boor, H.S. Leipner and P. Werner, J. Phys. Chem. C, 2012, 116, 13446-13451.

23. X. Zhong, Y.Q. Qu, Y.C. Lin, L. Liao and X.F. Duan, ACS Appl. Mater. Interfaces, 2011, 3, 261270.

24. S. Ciampi, J.B. Harper and J.J. Gooding, Chem. Soc. Rev., 2010, 39, 2158-2183. 
25. J.M. Buriak, Chem. Commun., 1999, 1051-1060.

26. Z.P. Huang, H. Fang and J. Zhu, Adv. Mater., 2007, 19, 744-748.

27. K. Peng, M. Zhang, A. Lu, N.-B. Wong, R. Zhang and S.-T. Lee, Appl. Phys. Lett., 2007, 90, 163123.

28. S. Ciampi, T. Böcking, K.A. Kilian, M. James, J.B. Harper and J.J. Gooding, Langmuir, 2007, 23, 9320-9329.

29. S. Ciampi, T. Böcking, K.A. Kilian, J.B. Harper and J.J. Gooding, Langmuir, 2008, 24, 5888-5892.

30. R. Boukherroub, J.T.C. Wojtyk, D.D.M. Wayner and D.J. Lockwood, J. Electrochem. Soc., 2002, 149, H59-H63.

31. F. Cattaruzza, A. Cricenti, A. Flamini, M. Girasole, G. Longo, A. Mezzi and T. Prosperi, J. Mater. Chem., 2004, 14, 1461-1468.

32. K. Balasundaram, J.S. Sadhu, J.C. Shin, B. Azeredo, D. Chanda, M. Malik, K. Hsu, J.A. Rogers, P. Ferreira, S. Sinha and X.L. Li, Nanotechnology, 2012, 23, 305304.

33. T.R. Chan, R. Hilgraf, K.B. Sharpless and V.V. Fokin, Org. Lett., 2004, 6, 2853-2855.

34. G.S. Hong, C. Li and L.M. Qi, Adv. Funct. Mater., 2010, 20, 3774-3783.

35. C. Li, G. Hong, P. Wang, D. Yu and L. Qi, Chem. Mater., 2009, 21, 891-897. 


\section{Chapter 6}

\section{Selective silicon nanowire functionalization: towards early cancer DNA detection}

Silicon nanowire chips can function as sensors for cancer DNA detection, whereby selective functionalization of the silicon sensing areas over the surrounding silicon oxide would prevent loss of analyte and thus increase the sensitivity. The thermal hydrosilylation of unsaturated carbon-carbon bonds onto $\mathrm{H}$-terminated silicon has been studied here to selectively functionalize the silicon nanowires with a monolayer of 1,8-nonadiyne. The silicon oxide areas, however, appeared to be functionalized as well. The selectivity towards the $\mathrm{Si}-\mathrm{H}$ regions was increased by introducing an extra HF treatment after the 1,8-nonadiyne monolayer formation. This step (partly) removed the monolayer from the silicon oxide regions, whereas the $\mathrm{Si}-\mathrm{C}$ bonds at the $\mathrm{Si}$ areas remained intact. The alkyne headgroups of immobilized 1,8-nonadiyne were functionalized with PNA probes by coupling azido-PNA and thiol-PNA by click chemistry and thiol-yne chemistry, respectively. Although both functionalization routes were successful, hybridization could only be detected on the samples with thiol-PNA. No fluorescence was observed when introducing dye-labelled noncomplementary DNA, which indicates specific DNA hybridization. These results open up the possibilities for creating silicon nanowire-based sensors for binding tumor DNA in both a specific way and with a higher sensitivity.

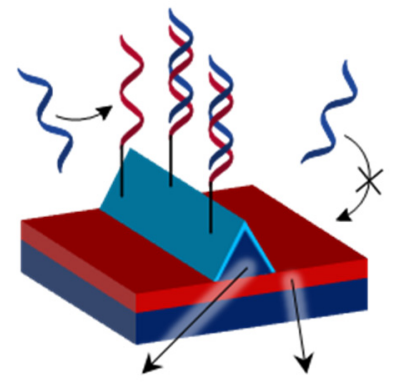

silicon silicon oxide 


\subsection{Introduction}

Early diagnostics of diseases, in particular cancer, is receiving increasing attention as early detection promises a higher cure rate and/or longer survival periods. ${ }^{1}$ Detecting tumor DNA is preferably noninvasive, for example based on blood ${ }^{2}$ or urine samples. ${ }^{3}$ As a sensor, lab-on-a-chip configurations are attractive since analysis outside the hospital is possible, for example at the general practitioner or even at home as a do-it-yourself test. ${ }^{4,5}$ Ideally, the DNA detection is highly specific, i.e., for cancer DNA only, in particular for a well-recognized marker sequence, and highly sensitive, i.e., detecting the cancer DNA at low concentrations, also in the presence of a lot of other background DNA.

Surface chemistry can be used to specifically capture tumor DNA. ${ }^{6,7}$ For this purpose, a specific DNA or peptide nucleic acid (PNA) oligo can be attached as a probe sequence to the surface, which consists of the complementary strand for the disease-specific DNA sequence. PNA is an artificially synthesized polymer that functions as a mimic for DNA and contains a neutral peptide-like backbone instead of a negatively charged deoxyribose phosphate backbone. ${ }^{8}$ The spacing between the nucleotides is equal for DNA and PNA, which makes PNA-DNA hybridization possible. For sensing purposes, PNA is preferred as a probe since i) PNA-DNA interactions are stronger than DNA-DNA interactions due to the absence of electrostatic repulsion and ii) PNA-DNA interactions often show a better selectivity. ${ }^{9}$ The absence of charge repulsion also prevents inhibition of DNA approach to the sensing surface, which then enables a higher sensitivity.

Sensors to detect PNA-DNA hybridization rely on signal transduction based on surface plasmon resonance ${ }^{10}$ or electrical measurements, ${ }^{11-14}$ for example, and are frequently based on silicon nanowires on a chip. In the literature, hydrosilylation chemistry, in which unsaturated carbon-carbon bonds are coupled onto oxide-free silicon surfaces, is commonly used for fabricating silicon sensors for DNA detection. ${ }^{11-15}$ Hydrosilylation is advantageous because the resulting $\mathrm{Si}-\mathrm{C}$ bonds are stable in aqueous environment, and the absence of an insulating silicon oxide layer improves the electrical contact with the underlying substrate and thus the sensitivity of the sensor as well. ${ }^{14}$ Nonetheless, any adsorption of analyte DNA outside the sensor area, either specific or nonspecific, would result in a loss of sensitivity. Therefore, the sensitivity of a DNA sensor device can be enormously increased when the probe is only bound at the sensing areas. Here, we focus on chips with silicon nanowires surrounded by silicon oxide, ${ }^{16}$ of which the silicon regions should be functionalized selectively with the probe DNA or PNA. Hydrosilylation could potentially be used to selectively functionalize the silicon nanowires over the silicon oxide surroundings, as has been suggested in the literature. ${ }^{15,17,18}$ This material-selective functionalization has, however, not yet been studied in detail. 
Here, we study the selective functionalization of the sensing area of a chip, i.e., its silicon nanowires, whereas the surrounding silicon oxide should not be functionalized. Specifically, the dialkyne 1,8-nonadiyne is coupled to $\mathrm{H}$-terminated silicon in order to achieve material-selective functionalization. Whereas most examples from literature are based on photochemical hydrosilylation, i.e., coupling under illumination by light, we have used thermal hydrosilylation since this technique generally yields monolayers with a higher coverage. ${ }^{19}$ The freestanding alkyne group of the 1,8-nonadiyne monolayer can then be functionalized with azide or thiol moieties by copper-catalyzed click chemistry ${ }^{20,21}$ and thiol-yne chemistry, ${ }^{22,23}$ respectively. First, the specific functionalization of silicon nanowires on a chip is tested by click chemistry with dummy molecules, i.e., with an azide-functionalized dye and with azide-functionalized nanoparticles to enable characterization by fluorescence microscopy and high-resolution scanning electron microscopy (HR-SEM), respectively (Section 6.2.1). Thereafter, azido-PNA or thiol-PNA is coupled onto the 1,8-nonadiyne monolayer as a proof of concept for a biosensor. PNA-DNA hybridization with cancer DNA is investigated using fluorescence microscopy and quartz crystal microbalance (QCM) measurements (Section 6.2.2).

\subsection{Results and discussion}

The chip designed for early cancer diagnostics is based on silicon nanowires as sensing areas surrounded by inactive silicon oxide $\left(\mathrm{SiO}_{\mathrm{x}}\right)$, as reported before (Scheme 6.1). ${ }^{16} \mathrm{~A}$ sensor consists of two silicon nanowires with a triangular cross section, bridging two contact pads. A substrate contained several sensors with the same design and different nanowire lengths. For proof of principle tests, chips with silicon-rich silicon nitride contact pads instead of metal contacts were used, ${ }^{16}$ which did not allow for electrical characterization. Instead, fluorescence microscopy, HR-SEM, and QCM were used to verify the selective functionalization routes. In the nanowire fabrication process, two rectangular areas of silicon oxide, next to the silicon nanowires, are slightly etched (Scheme 6.1). The composition of these areas is similar to the surrounding silicon oxide, but the difference in thickness can be seen as a contrast in the microscopy images.

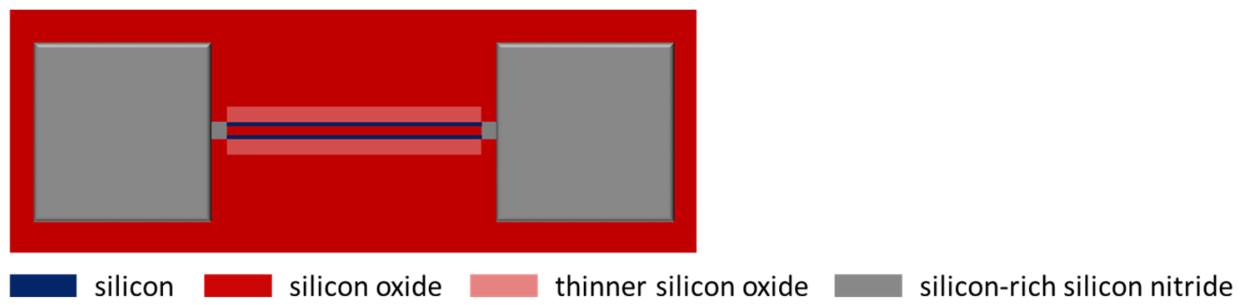

Scheme 6.1. Schematic illustration of the chips with silicon nanowires. 
The process to functionalize the silicon nanowires specifically with probe PNA, while not functionalizing the silicon oxide areas, is shown in Scheme 6.2. First, 4 min immersion in an aqueous solution of $1 \%$ hydrofluoric acid (HF) leads to removal of the thin oxide layer from the silicon nanowires. The surroundings, including the rectangular areas around the nanowires, consist of a thick $(>120 \mathrm{~nm})$ silicon oxide layer, which is only marginally removed by the HF dip. Nonetheless, the treatment needs to be controlled well to avoid underetching, and thereby potential removal, of the $\mathrm{Si}$ nanowires. Subsequent monolayer formation with 1,8-nonadiyne only applies onto the $\mathrm{H}$-terminated silicon nanowires. Functionalized PNA can be coupled thereafter onto the alkyne headgroup by click chemistry with azido-PNA ${ }^{24,25}$ or thiol-yne chemistry with thiol-PNA. ${ }^{26,27}$ Introducing complementary DNA (cDNA) onto this chip should result in specific and spatioselective binding onto the probe-functionalized nanowires. Since cDNA only adsorbs onto the sensing areas of the chip, tumor DNA detection should thereby be possible with a higher sensitivity.

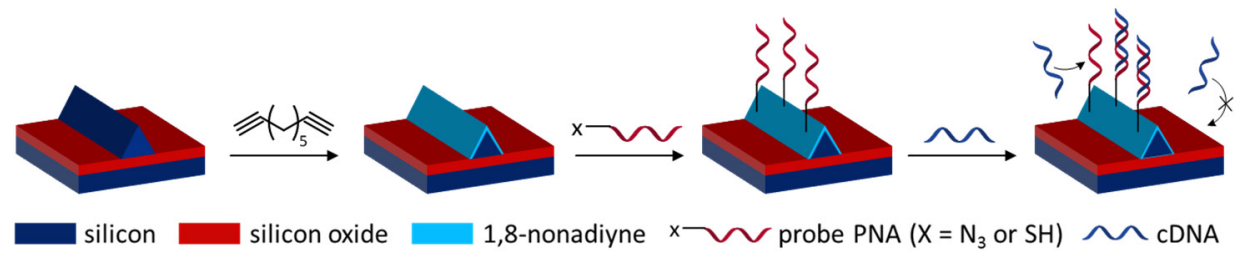

Scheme 6.2. Schematic illustration of the material-selective monolayer formation and subsequent probe PNA modification onto $\mathrm{H}$-terminated silicon nanowires surrounded by silicon oxide.

\subsubsection{Material-selective monolayer formation}

Selective monolayer formation at the silicon nanowires was tested using click chemistry with dummy compounds (Scheme 6.3). As stated above, a monolayer of 1,8-nonadiyne is first formed onto H-terminated silicon nanowires. Click chemistry with an azidefunctionalized dye or azide-functionalized gold nanoparticles (NPs) was used to allow characterization by fluorescence microscopy and HR-SEM, respectively, in order to probe the success and the selectivity of the preceding monolayer formation step. 
a
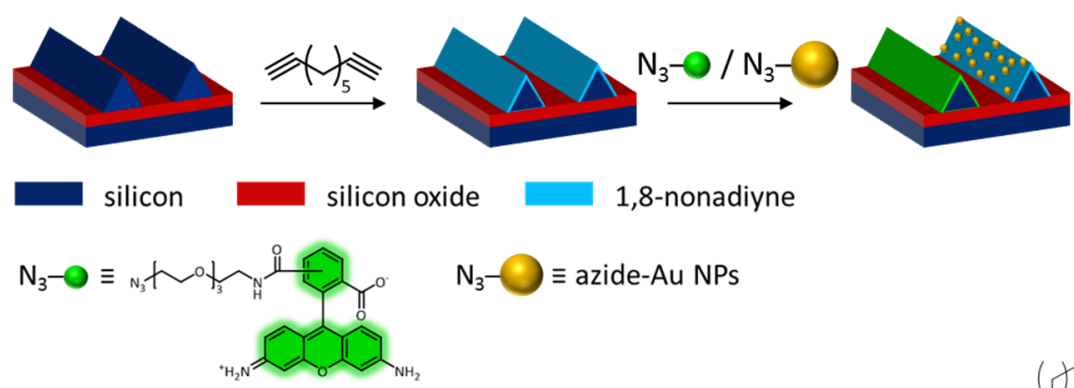

b

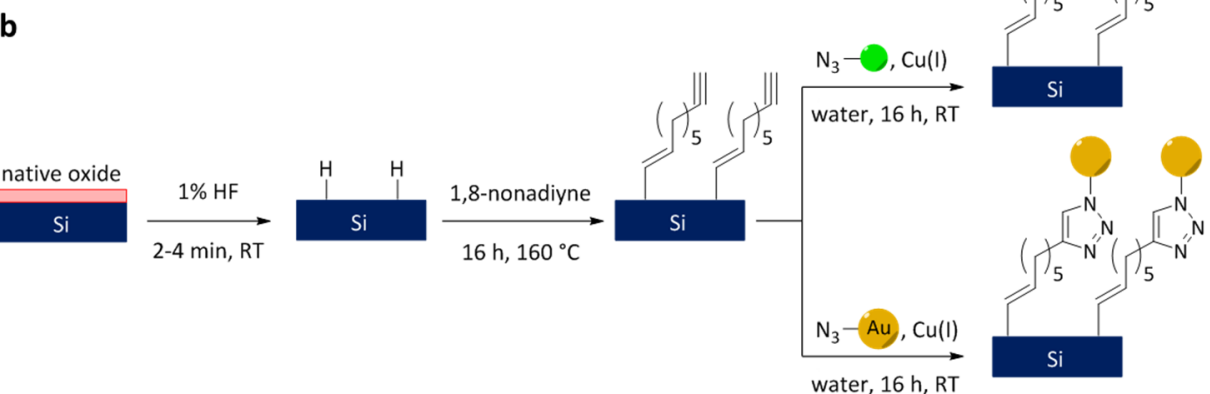

Scheme 6.3. a) Schematic illustration and b) reaction scheme of the click chemistry routes tested at silicon nanowires functionalized with a 1,8-nonadiyne monolayer.

To properly discriminate between monolayer formation at the $\mathrm{Si}$ and $\mathrm{SiO}_{\mathrm{x}}$ regions, first tests were performed on patterns larger than the nanowires on chip (150 nm diameter). Using photolithography, patterns of silicon oxide dots were created with a diameter of $100 \mu \mathrm{m}$ and a thickness of $160 \mathrm{~nm}$, surrounded by $\mathrm{Si}-\mathrm{H}$ due to silicon oxide removal on these resist-free areas (Scheme 6.4). Immediately afterwards, a monolayer of 1,8-nonadiyne was formed on the patterned substrate as described in Chapter 3, i.e., by thermal hydrosilylation $\left(160^{\circ} \mathrm{C}\right)$ of the pure 1,8-nonadiyne. After click chemistry with an azide-functionalized dye, fluorescence imaging was expected to show nonfluorescent silicon oxide dots, surrounded by fluorescent silicon. Figure 6.1a showed, however, the inverted pattern with a higher intensity at the dots compared to the surrounding silicon. This observation does not necessarily mean that the coverage of the dye is higher at the dots, as silicon is known to heavily quench fluorescence signals. ${ }^{28}$

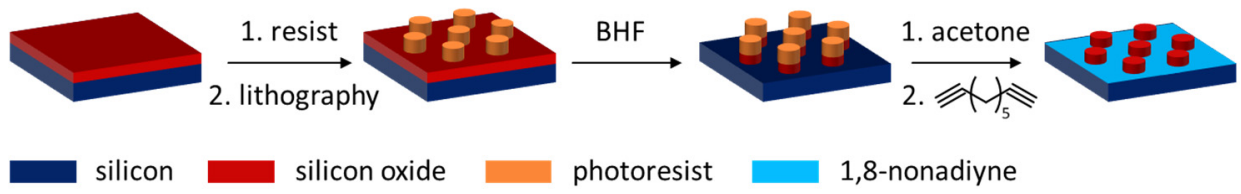

Scheme 6.4. Schematic illustration of the formation of silicon oxide dots surrounded by $\mathrm{H}$-terminated silicon and subsequent material-selective monolayer formation (BHF = buffered hydrogen fluoride). 


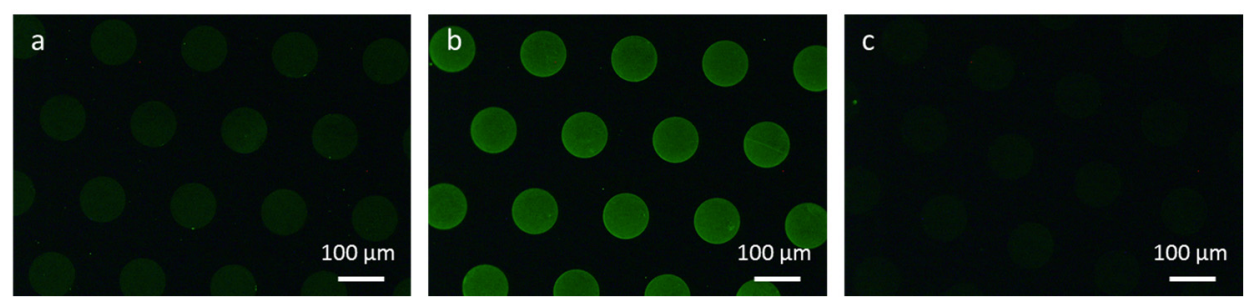

Figure 6.1. Fluorescence microscopy images (exposure time $1 \mathrm{~s}$ ) of $\mathrm{SiO}_{2} / \mathrm{Si}$ patterns functionalized with a) 1,8-nonadiyne and azide-functionalized dye, b) the same sequence as (a) with an extra BHF $\operatorname{dip}(10 \mathrm{~s})$ after the 1,8-nonadiyne monolayer formation, and c) a control sample without 1,8-nonadiyne.

X-ray photoelectron spectroscopy (XPS) was chosen as a more detailed characterization method on the selective monolayer formation, as element scans could distinguish between unbound azide groups and triazole moieties after click chemistry. XPS elemental mapping showed more $\mathrm{O}$ atoms at the dots, as expected due to the $\mathrm{SiO}_{2}$ composition (Figure 6.2a). The expected contrast in $\mathrm{C}$ and $\mathrm{N}$ was, however, hardly distinguishable. This cannot be due to physisorption of the dye, as a control sample without a 1,8-nonadiyne monolayer did not show any fluorescence (Figure 6.1c) nor $\mathrm{N}$ atoms (Figure 6.2c) by fluorescence microscopy and XPS, respectively. We therefore attribute these observations to, here undesired, 1,8-nonadiyne monolayer formation at the $\mathrm{SiO}_{2}$ dots. This nonselective functionalization of oxidized and unoxidized silicon by hydrosilylation has been observed before in the literature. ${ }^{29}$ This was supported by a change in the contact angle of a planar $\mathrm{SiO}_{2}$ substrate from hydrophilic $\left(<20^{\circ}\right)$ after a $1 \% \mathrm{HF}$ dip to $77.8^{\circ} \pm 1.2$ after the 1,8 -nonadiyne reaction, which is comparable to a 1,8 -nonadiyne monolayer on $\mathrm{Si}-\mathrm{H}$ (Section 6.2.2, vide infra). In the literature a 2 min $2 \% \mathrm{HF}$ dip was sufficient to remove the monolayer from the oxidized regions. ${ }^{29}$ Here, a buffered hydrogen fluoride (BHF) dip for $10 \mathrm{~s}$ lowered the contact angle to $<20^{\circ}$, which indicates removal of any undesired monolayer at the silicon oxide parts. The $\mathrm{Si}-\mathrm{C}$ bound monolayer should withstand this treatment, as observed in Chapter 5 where comparable HF concentrations were used. 


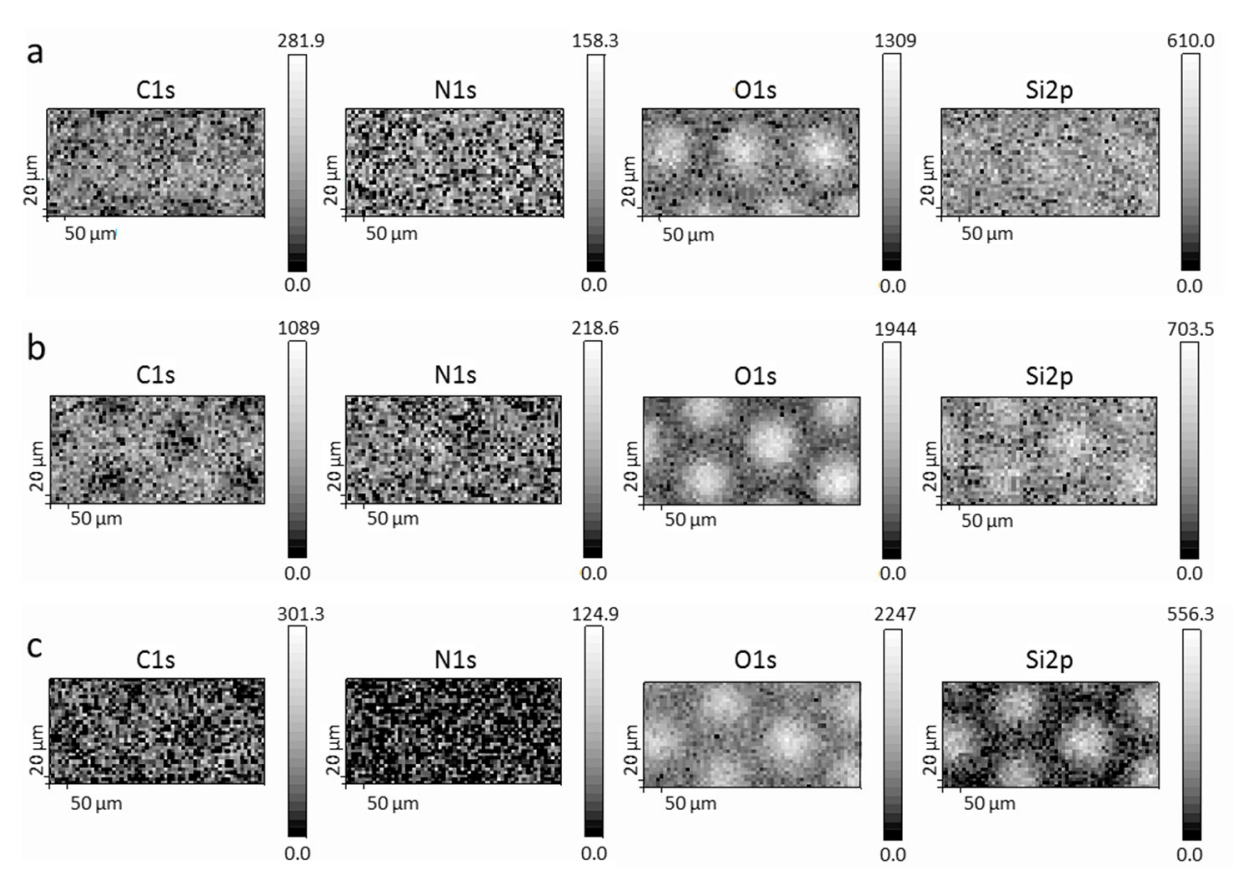

Figure 6.2. Elemental mapping of the $\mathrm{C} 1 \mathrm{~s}, \mathrm{~N} 1 \mathrm{~s}, \mathrm{O} 1 \mathrm{~s}$, and $\mathrm{Si} 2 \mathrm{p}$ regions on the $\mathrm{SiO}_{2} / \mathrm{Si}$ patterned substrates of a) Figure 6.1a, b) Figure 6.1b, and c) Figure 6.1c.

When an extra BHF dip was performed between the 1,8-nonadiyne monolayer formation and the click chemistry step on a patterned sample, an even higher fluorescence intensity was observed at the dots (Figure 6.1b). Nonetheless, the XPS elemental mapping showed more $\mathrm{C}$ and $\mathrm{Si}$ at the areas outside the dots (Figure 6.2b), as expected from the selective presence of a 1,8-nonadiyne monolayer. The difference in composition was also reflected by the Si2p element spectra, in which oxidized Si was observed at the dots (Figure 6.3a) and mainly unoxidized Si outside the dots (Figure 6.3b). For the N1s signal, however, hardly a difference could be detected between the $\mathrm{Si}$ and $\mathrm{SiO}_{2}$ regions in the mappings (Figure 6.2b). The element spectra recorded at (Figure 6.3c) and outside (Figure 6.3d) the dots showed the presence of $\mathrm{N}$ atoms at both areas. The highest intensity was observed at the $\mathrm{Si}$ areas, as expected. The presence of $\mathrm{N}$ atoms at the $\mathrm{SiO}_{2}$ dots still denotes physisorption of dye or undesired 1,8-nonadiyne monolayer formation (and subsequent click chemistry) at these regions, even though the BHF dip should have removed the monolayer selectively. The deconvoluted spectra showed two bands at 399 and $402 \mathrm{eV}$ in the N1s region for both areas (Figure 6.3c, d), which are characteristic for the formation of a triazole moiety. Any physisorbed azide-containing compound would have appeared at $405 \mathrm{eV},{ }^{21}$ which was not observed in these spectra. All these observations denote undesired 1,8-nonadiyne monolayer formation at the $\mathrm{SiO}_{2}$ dots, although in a lesser extent than the desired monolayer formation at the Si areas. 

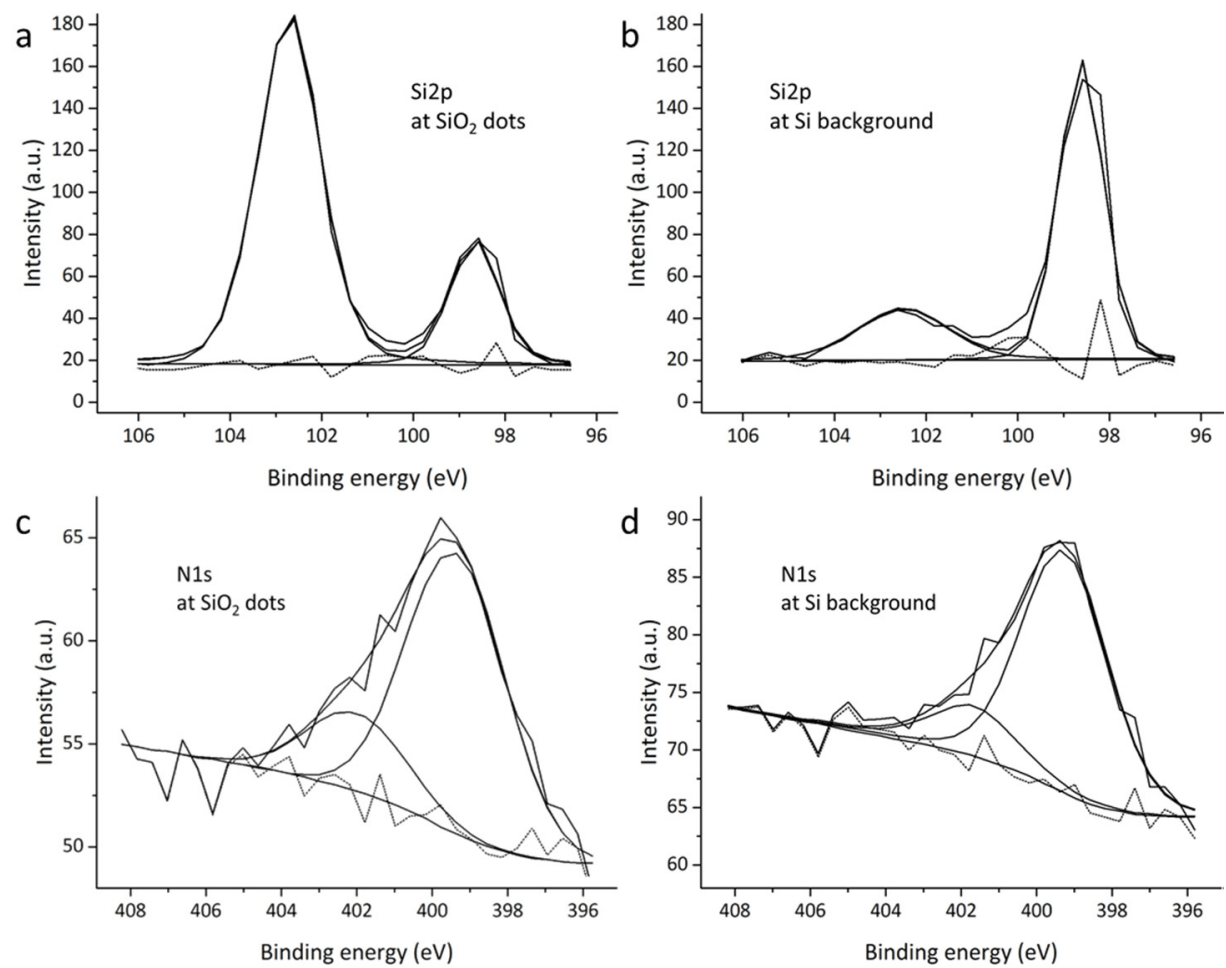

Figure 6.3. XPS spectra of the $\mathrm{SiO}_{2} / \mathrm{Si}$ patterned substrates of Figure $6.1 \mathrm{~b}$, including a) the $\mathrm{Si} 2 \mathrm{p}$ region at the $\mathrm{SiO}_{2}$ dots, b) the $\mathrm{Si} 2 \mathrm{p}$ region at the surrounding $\mathrm{Si}, \mathrm{c}$ ) the $\mathrm{N} 1 \mathrm{~s}$ region at the $\mathrm{SiO}_{2}$ dots, and d) the N1s region at the surrounding Si.

The monolayer formation process was transferred onto chips with silicon nanowires surrounded by silicon oxide. After 1,8-nonadiyne monolayer formation, click chemistry was performed with an azide-functionalized dye or $10 \mathrm{~nm}$ azide-functionalized Au NPs. Both fluorescence microscopy (Figure 6.4a, d) and HR-SEM images (Figure 6.4e, f) showed successful functionalization of the silicon nanowires, as indicated by a bright fluorescence and dots with a bright contrast in the HR-SEM images, respectively. A control sample without 1,8-nonadiyne was only slightly fluorescent after click chemistry (Figure 6.4c, d), although no fluorescent signal was expected at all. For the HR-SEM image, an energyselective backscatter (ESB) detector was used to display compositional variations on the sample based on atomic number (Figure 6.4f). Whereas these HR-SEM images do seem to show specific functionalization, the background fluorescence observed at the oxidized areas in Figure 6.4a could denote nonspecific physisorption and/or undesired 1,8-nonadiyne monolayer formation, as observed above for the $\mathrm{SiO}_{2}$ dots pattern. The higher background fluorescence in the rectangular areas around the silicon nanowires is expected to be due to a higher surface roughness, which could lead to a higher monolayer coverage. An extra BHF dip between the 1,8-nonadiyne monolayer formation and the 
click chemistry step resulted in a lower fluorescence signal of the $\mathrm{SiO}_{x}$ background and showed more defined presence of the dye at the nanowires only (Figure 6.4b, d). Thus, material-selective functionalization at the nanowires seems to be possible, although removal of 1,8-nonadiyne from the silicon oxide areas is a necessary step.
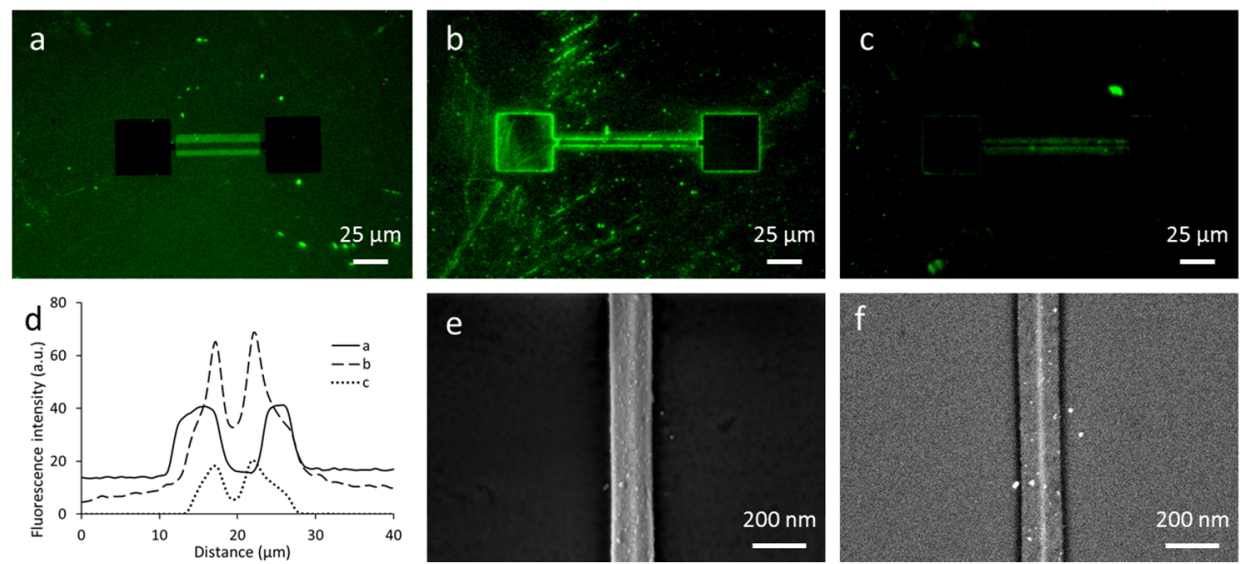

Figure 6.4. Selective functionalization of silicon nanowires on chips with a 1,8-nonadiyne monolayer characterized by a-d) fluorescence microscopy after click chemistry with an azide-functionalized dye (exposure time $2 \mathrm{~s}$ ), and e-f) HR-SEM imaging after click chemistry with azide-functionalized Au NPs. The fluorescence microscopy images include a) a chip treated with 1,8-nonadiyne and azidefunctionalized dye, b) a chip treated additionally with a $10 \mathrm{~s}$ BHF dip after the 1,8-nonadiyne monolayer formation, c) a control sample without 1,8-nonadiyne, and d) the corresponding fluorescence intensity profiles. The HR-SEM images include e) an InLens zoom-in image of a silicon nanowire and f) the corresponding ESB image to show a contrast in elements.

\subsubsection{PNA-DNA hybridization}

In order to use the chips for cancer DNA detection, surface chemistry can be used to provide specific hybridization. Tests on PNA-DNA hybridization were first performed on planar silicon substrates. A monolayer of 1,8-nonadiyne was formed on $\mathrm{H}$-terminated $\mathrm{Si}$ as sketched above (Scheme 6.3b). Subsequently, two functionalization routes were used to couple PNA probes onto the freestanding alkyne moiety, i.e., click chemistry with azido-PNA (Scheme 6.5a) and thiol-yne chemistry with thiol-PNA (Scheme 6.5b). In the latter reaction, potentially two thiol groups can bind to one alkyne headgroup. ${ }^{23}$ 
a

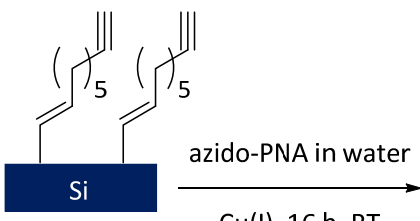

$\mathrm{Cu}(\mathrm{l}), 16 \mathrm{~h}, \mathrm{RT}$
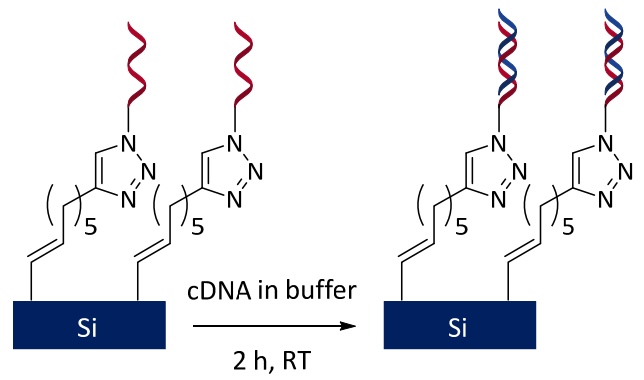

$\mathrm{Si}$

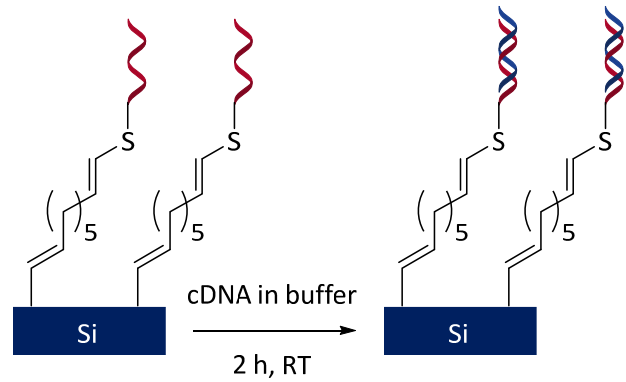

$2 \mathrm{~h}, \mathrm{RT}$

b

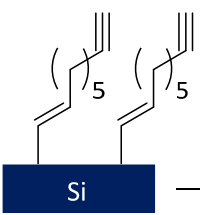
thiol-PNA in PBS

$365 \mathrm{~nm}, 1 \mathrm{~h}, \mathrm{RT}$

Scheme 6.5. Reaction schemes of a) click chemistry with azido-PNA and b) thiol-yne chemistry with thiol-PNA onto a 1,8-nonadiyne monolayer, followed by hybridization with complementary DNA.

Click chemistry was performed as described in Section 6.2.1 and resulted in a change of the contact angle from $78.3^{\circ} \pm 2.2$ for a 1,8 -nonadiyne monolayer to $50.1^{\circ} \pm 1.4$ after azido-PNA coupling. This lowering in contact angle indicates azido-PNA coupling to the surface, as the increased hydrophilicity is expected from the polar structural groups. ${ }^{30}$ Thiol-yne chemistry was performed by exposing the 1,8-nonadiyne monolayer to a solution of thiol-PNA in phosphate-buffered saline (PBS) under illumination with a $365 \mathrm{~nm}$ light source. The thiol-PNA-functionalized surface changed the contact angle from $87.6^{\circ} \pm 1.1$ after 1,8 -nonadiyne to $46.5^{\circ} \pm 3.2$ after thiol-PNA, again indicating a hydrophilic surface and thus proper functionalization. XPS measurements confirmed the coupling of PNA for both routes by the atomic percentages of $\mathrm{N}$ and $\mathrm{S}$, which elements are absent in the 1,8-nonadiyne monolayer, but increase to $16 \% \mathrm{~N}$ after click chemistry (each azido-PNA molecule contains $94 \mathrm{~N}$ atoms) and $0.26 \% \mathrm{~S}$ after thiol-yne chemistry (each thiol-PNA molecule contains $1 \mathrm{~S}$ atom). Thus, azido-PNA and thiol-PNA have been successfully coupled onto 1,8-nonadiyne monolayers.

Hybridization tests (Scheme 6.5) were performed at micrometer-sized lines of azido-PNA and thiol-PNA to be able to visualize hybridization with dye-labeled cDNA by a contrast in the fluorescence signal. On a fully formed 1,8-nonadiyne monolayer, lines of PNA were created by microcontact printing. Azido-PNA was microcontact printed using $\mathrm{Cu}(\mathrm{I})\left(\mathrm{CH}_{3} \mathrm{CN}\right)_{4} \mathrm{PF}_{6}$ and TBTA as stabilizing ligand, ${ }^{31}$ as opposed to the use of a $\mathrm{Cu}(\mathrm{II})$ salt with ascorbic acid for the click chemistry reactions described above. Seen the different 
procedure, XPS was used again to verify whether the azido-PNA coupling was successful. On a separate sample, an atomic percentage of $12 \% \mathrm{~N}$ was observed after microcontact printing, which indicates a successful coupling. The yield of the click reaction is comparable to the $\mathrm{Cu}(\mathrm{II})$ reaction described above $(16 \% \mathrm{~N})$ when taking into account the maximum coverage of $2 / 3$ due to the spacing of the microcontact printing stamp ( $10 \mu \mathrm{m}$ diameter, $5 \mu \mathrm{m}$ spacing) and the use of a different azido-PNA sequence. For microcontact printing of the thiol-yne reaction, ${ }^{22}$ the stamp with lines ( $5 \mu \mathrm{m}$ diameter, $3 \mu \mathrm{m}$ spacing) was inked with a thiol-PNA solution in PBS, equal to the samples that were fully immersed. As a difference, the substrate was illuminated through the stamp. After microcontact printing, hybridization with a fluorescently labeled cDNA (dye-cDNA, rhodamine) did not result in the expected fluorescent pattern for the substrate with azido-PNA (Figure 6.5a, c). For the samples functionalized with thiol-PNA, however, fluorescent lines were observed after hybridization (Figure 6.5b, c).
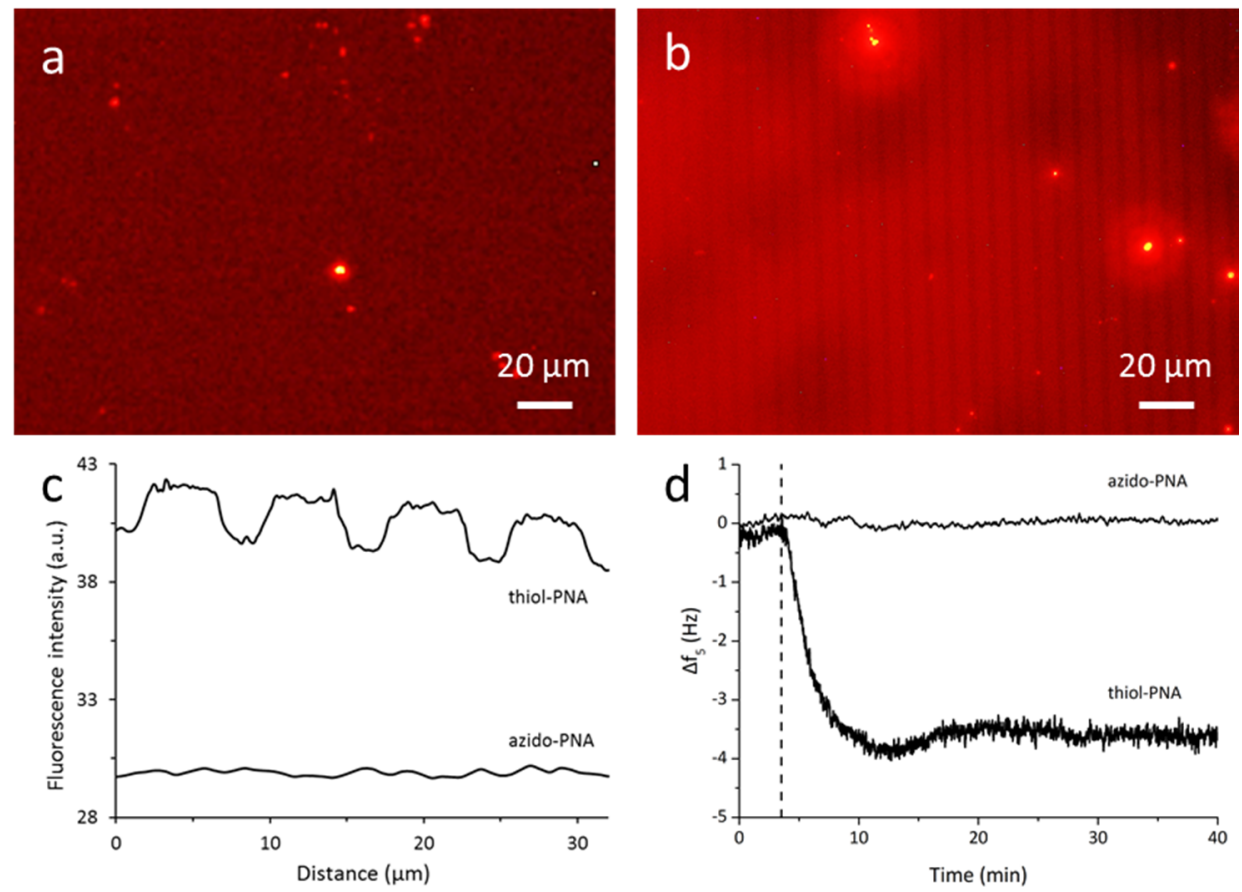

Figure 6.5. a,b) Fluorescence microscopy images (brightness increased by $20 \%$, contrast increased by $40 \%$, exposure time $20 \mathrm{~s}$ ) after hybridization with dye-cDNA on silicon substrates with a 1,8-nonadiyne monolayer functionalized by microcontact printing of a) azido-PNA and $b$ ) thiol-PNA. c) The corresponding fluorescence intensity profiles of the original images. d) QCM-D measurements on silicon sensors with azido-PNA or thiol-PNA attached to a 1,8-nonadiyne monolayer, showing the fifth resonance frequency overtone $\left(\Delta f_{5}\right)$ when adding a $3 \mu \mathrm{M} c D N A$ (azido-PNA) or $2 \mu \mathrm{M}$ cDNA (thiol-PNA) solution in buffer; the vertical dashed line indicates the time at which the flow of cDNA was started. 
The hybridization step was quantified further using quartz crystal microbalance with dissipation monitoring (QCM-D), where a decrease in resonance frequency reflects an increase in mass at the surface. The frequency was monitored while flowing cDNA over PNA-functionalized QCM sensors (Figure 6.5d). These measurements supported the observations of the fluorescence microscopy images. No hybridization was observed for the azido-PNA surface, whereas the thiol-PNA-functionalized QCM sensor showed a decrease of the resonance frequency upon addition of a $2 \mu \mathrm{M}$ cDNA solution. This reflects successful PNA-DNA hybridization for the thiol-PNA substrates. As a rough estimation, the Sauerbrey equation was used to convert the observed frequency change $(3.4 \mathrm{~Hz})$ into a mass change, giving an adsorbed mass of about $12 \mathrm{ng} / \mathrm{cm}^{2}$. In the best case, i.e., assuming no water adsorption, this mass change corresponds to a cDNA coverage of about $10^{-12} \mathrm{~mol} / \mathrm{cm}^{2}$, which is comparable to values reported before in the literature for PNA/DNA hybridization at surfaces. ${ }^{10,32}$ Considering the azido-PNA substrates, the reason for the absence of hybridization is unknown, as the presence of azido-PNA was confirmed by XPS. Two different azido-PNA sequences were tested, including a sequence similar to the thiol-PNA oligonucleotide, which was expected to be successful seen the positive microcontact printing and QCM-D results. Backfilling of the 1,8-nonadiyne monolayer with azide-functionalized tetra(ethylene glycol) as antifouling layer did not improve the results either.

The successful hybridization on thiol-PNA samples described above was transferred onto silicon nanowire chips. After applying the same functionalization route to couple thiol-PNA onto a 1,8-nonadiyne monolayer, hybridization with dye-functionalized DNA was characterized using fluorescence microscopy. This resulted in a clear fluorescence signal after immersion in a dye-cDNA solution (Figure 6.6a, d), as observed in Figure 6.4a. When adding a dye-functionalized noncomplementary DNA (dye-ncDNA) onto the PNA monolayer, no fluorescence could be detected (Figure 6.6b, d), which indicates that the PNA-DNA interactions are specific. A control sample without 1,8-nonadiyne monolayer did not show fluorescence either after immersion in dye-cDNA (Figure 6.6c, d), which indicates that there is no physisorption of dye-cDNA in the absence of PNA. Consequently, the fluorescence observed in Figure 6.6a, in particular in the $\mathrm{SiO}_{x}$ areas, is likely due to the, here undesired, presence of a 1,8-nonadiyne monolayer with PNA at the surrounding silicon oxide. 

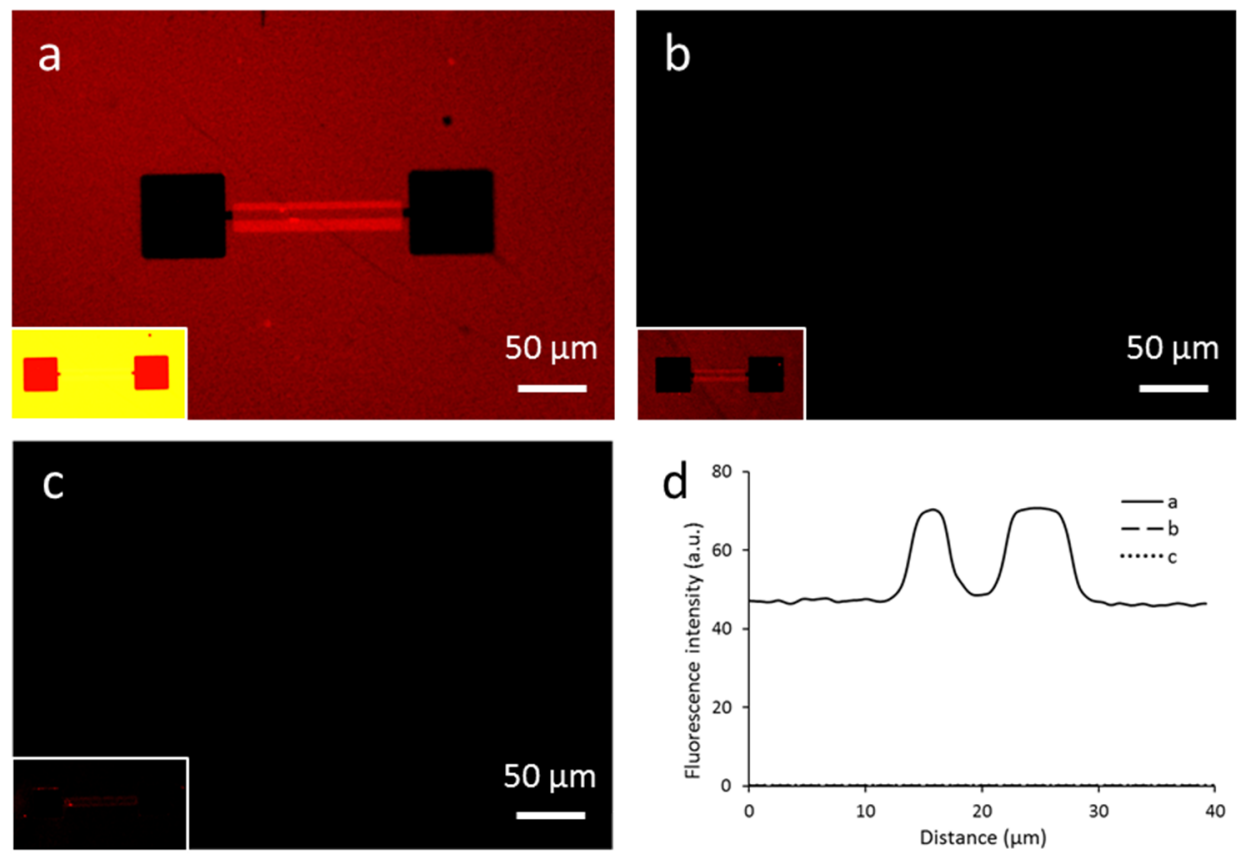

Figure 6.6. a-c) Fluorescence microscopy images of silicon nanowires on chips functionalized with a 1,8-nonadiyne monolayer and thiol-PNA, after adding a) dye-cDNA, b) dye-ncDNA, and c) a control sample without 1,8-nonadiyne, immersed in dye-cDNA. The exposure time is $50 \mathrm{~ms}$ for the main images and $2 \mathrm{~s}$ for the insets. d) The corresponding fluorescence intensity profiles of the main images, where the profiles of (b) and (c) are located at zero intensity.

\subsection{Conclusions and outlook}

In summary, selective functionalization of silicon nanowire sensors with probe PNA appeared impossible in a direct way. Hydrosilylation of 1,8-nonadiyne led to a covalently bound monolayer at both the $\mathrm{Si}-\mathrm{H}$ and the $\mathrm{SiO}_{x}$ regions, as shown by fluorescence microscopy and XPS after click chemistry with an azide-functionalized dye. An extra BHF dip after 1,8-nonadiyne monolayer formation was used to partly remove the monolayer from the oxidized regions. This seemed to result in successful local functionalization at the silicon nanowires only, although the BHF treatment only resulted in a minor contrast between the $\mathrm{Si}$ and $\mathrm{SiO}_{2}$ regions for surfaces patterned at a larger scale. The reason for this apparent difference between the substrates is still unknown. Thus, selective functionalization of silicon over silicon oxide seems to be possible when using an extra (B) HF treatment, but this step requires more optimization to increase the selectivity.

Monolayers of 1,8-nonadiyne functionalized with probe PNA were used to test the hybridization with complementary DNA (cDNA) at the surface. Azido-PNA and thiol-PNA were successfully coupled onto the 1,8-nonadiyne monolayer, as confirmed by contact angle and XPS measurements. For unknown reasons, no hybridization could be detected 
on the samples with azido-PNA. Nonetheless, successful hybridization of cDNA onto the substrates with thiol-PNA was confirmed by fluorescence microscopy and QCM-D measurements. On chips, hybridization was only observed when using cDNA and not for the noncomplementary variant, which indicates specificity towards a disease-specific DNA sequence. More tests would be required to verify whether single mismatch DNA does not lead to hybridization either, as this could comprise the difference between healthy and unhealthy DNA. Next, PNA/DNA hybridization has to be tested on chips with selective monolayer formation, i.e., with a (B)HF dip after 1,8-nonadiyne monolayer formation, as applied in Section 6.2.1.

To increase the selectivity of the 1,8-nonadiyne monolayer formation on silicon, the surrounding silicon oxide could first be functionalized with a silane-functionalized antifouling compound, for example poly(ethylene glycol) silane. This reaction should not occur at the $\mathrm{Si}-\mathrm{H}$ regions, so that the second step could be used to backfill the $\mathrm{Si}-\mathrm{H}$ regions. The creation of $\mathrm{Si}-\mathrm{H}$ is then the most crucial step, since the HF dip should occur before the silane formation and the $\mathrm{Si}-\mathrm{H}$ moieties should remain for a prolonged period of time. Alternatively, a one-step reaction could be performed with a mixture of silanebased and alkyne-based molecules, which preferably graft onto the oxidized and unoxidized regions, respectively. ${ }^{17}$ In addition, the thermal hydrosilylation route could be replaced by the photochemical version. Mischki et al. observed no difference in contact angle when reacting $\mathrm{Si}-\mathrm{H}$ and oxidized silicon with 1-decene under thermal conditions $\left(102^{\circ}\right.$ for both areas), whereas the photochemical route showed a contact angle of $91^{\circ}$ at the $\mathrm{Si}-\mathrm{H}$ regions and $59^{\circ}$ at the oxidized areas. ${ }^{29}$ Alternatively, the reaction could be performed in the dark, since the oxidized areas then showed an even lower contact angle $\left(33^{\circ}\right)$, whereas 1 -alkynes could still react onto $\mathrm{Si}-\mathrm{H}$ with relatively high yield. ${ }^{33}$

Next, the chip design could be used to increase the selectivity of the monolayer formation. One approach applies a potential at the nanowires to locally activate a homogeneously formed monolayer. In this way, a hydroquinone monolayer at silicon nanowires can be oxidized locally, for example, after which the next functionalization step is only applicable to these oxidized molecules. ${ }^{34}$ Another approach applies a bias onto the silicon nanowires to locally remove a protective polymer from the surface, which is called nanoscale Joule heating. ${ }^{35,36}$

All in all, these suggestions can be used for a more in-depth research on the selective 1,8-nonadiyne monolayer formation at the silicon nanowires. A proof of principle was shown for PNA/DNA hybridization after thiol-PNA coupling, which is required to further develop the silicon nanowire sensor. Herein, the $\mathrm{Si}-\mathrm{C}$ bound monolayer is beneficial to increase the specificity by using a specific marker sequence and to improve the sensitivity by avoiding an insulating silicon oxide layer. Further research is needed to validate 
whether the tumor DNA can be detected at concentrations low enough for early diagnostics and whether the tumor DNA can be detected in physiological solutions, i.e., in the presence of a lot of other background DNA.

\subsection{Acknowledgments}

The BIOS group (University of Twente) is kindly thanked for supplying the chips with silicon nanowires. From this group, Rik Rurup is gratefully acknowledged for fruitful discussions, proofreading of the manuscript, and his help with the hybridization experiments and general biology-related questions. Raymond Steen is gratefully acknowledged for performing the initial experiments on selective functionalization. Songyue Chen and Jan van Nieuwkasteele are acknowledged for sharing their experience on the chip design. Roberto Corradini and co-workers (Department of Chemistry, University of Parma) are gratefully acknowledged for the synthesis of the azido-PNA and thiol-PNA. Roberto Ricciardi is kindly thanked for his help with the QCM-D experiments and sharing his experience on the PNA/DNA hybridization. Wouter Vijselaar is gratefully acknowledged for growing the silicon oxide layer used for the patterned substrates. Carlo Nicosia is thanked for the synthesis of TBTA.

\subsection{Experimental section}

\subsubsection{Materials}

Boron-doped p-type silicon wafers (<100>-oriented, $100 \mathrm{~mm}$ diameter, single side polished, resistivity 5-10 $\Omega \cdot \mathrm{cm}$, thickness $525 \mu \mathrm{m}$ ) were obtained from Okmetic (Finland). Chips with silicon nanowires were fabricated as reported before ${ }^{16}$ and consisted of two silicon nanowires with a triangular cross section, bridging two silicon-rich silicon nitride contact pads surrounded by silicon oxide. Chips without metal contacts were used, which did not allow for electrical characterization. Silicon-coated QCM sensors QSX-Si, consisting of gold electrodes with $200 \mathrm{~nm}$ sputtered, polycrystalline silicon (resonance frequency of $5 \mathrm{MHz}$ ), were obtained from LOT-QuantumDesign $\mathrm{GmbH}$.

Acetone (pure, VWR), acetonitrile (ACS grade, $\mathrm{CH}_{3} \mathrm{CN}$, Merck), L-ascorbic acid (>99\%, Sigma-Aldrich), azide-fluor 488 (>90\%, Sigma-Aldrich), buffered hydrogen fluoride (VLSI, BHF, 7:1, Technic France), copper(II) sulfate pentahydrate (99.995\% metals basis, $\mathrm{Cu}(\mathrm{II}) \mathrm{SO}_{4} .5 \mathrm{H}_{2} \mathrm{O}$, Sigma-Aldrich), dimethyl sulfoxide (anhydrous, $>99.9 \%$, DMSO, SigmaAldrich), ethanol (absolute, VWR), ethylenediaminetetraacetic acid disodium salt dihydrate (>99\%, EDTA, Sigma-Aldrich), hydrofluoric acid 1\% (aqueous, VLSI, Technic France), hydrogen peroxide (33\%, $\left.\mathrm{H}_{2} \mathrm{O}_{2}, V W R\right), O$-(2-azidoethyl)-O'-methyl-triethylene glycol (azido-TEG, >90\%, Sigma-Aldrich), phosphate-buffered saline powder $(\mathrm{pH} 7.4$, results in $10 \mathrm{mM}$ PBS with $0.138 \mathrm{M} \mathrm{NaCl}$, Sigma-Aldrich), photoresist OiR 906-12 or OiR 907-17 (Fujifilm), resist developer OPD 4262 (Fujifilm), sodium chloride (>99.5\%, NaCl, 
Sigma-Aldrich), sodium citrate monobasic (>99\%, Sigma-Aldrich), sodium dodecyl sulfate (SDS, >99\%, Sigma-Aldrich), sulfuric acid (95\%, $\mathrm{H}_{2} \mathrm{SO}_{4}, \mathrm{VWR}$ ), tetrakis(acetonitrile)copper(I) hexafluorophosphate $\left(\mathrm{Cu}(\mathrm{I})\left(\mathrm{CH}_{3} \mathrm{CN}\right)_{4} \mathrm{PF}_{6}\right.$, Sigma-Aldrich), tris(2carboxyethyl)phosphine hydrochloride (TCEP, Sigma-Aldrich), and Tween-20 (Aldrich) were used as received. SSC buffer $20 \times$ consisted of $3 \mathrm{M}$ sodium chloride and $0.3 \mathrm{M}$ sodium citrate at $\mathrm{pH} 7.0$ in water. Tris-(benzyltriazolylmethyl)amine (TBTA) was synthesized according to a procedure from the literature. ${ }^{37}$ Hexane was obtained from a solvent purification system (MB SPS-800). Milli-Q water with a resistivity $>18 \mathrm{M} \Omega \cdot \mathrm{cm}$ was obtained from a Milli-Q Integral water purification system (Merck Millipore). Glassware used for the hydrosilylation reaction was dried overnight at $120^{\circ} \mathrm{C}$. The dialkyne 1,8-nonadiyne (98\%, Sigma-Aldrich) was dried over molecular sieves (0.3 nm). Dichloromethane (99.7\%, Actu-All) was dried over anhydrous magnesium sulphate (Merck). Azide-functionalized gold NPs of $10 \mathrm{~nm}$ diameter were obtained from NanoCS, with a particle concentration of $0.5 \mathrm{mg} / \mathrm{mL}$ in water (based on gold salt, $2.8 \times 10^{13}$ particles $/ \mathrm{mL}$ ), a size distribution $<15 \%$, and a poly(ethylene glycol) linker between the NPs and the azide groups.

The PNA sequences were synthesized by solid phase methodologies as reported before ${ }^{10}$ and included X-R-R-GCA GCG CGT TGG CAC-Gly-NH $\mathrm{N}_{2}$ as azido-PNA $1297 \mu \mathrm{M}$ in water, $X=2$-azide-acetic acid, $R=$ [2-(2-aminoethoxy)ethoxy]acetic acid, azide group at the $\mathrm{N}$ terminus $\left(5^{\prime}\right)$, probe for bladder cancer), X-R-R-CTA CGC CAC CAG CT-NH ${ }_{2}$ as azido-PNA 2 ( $272 \mu \mathrm{M}$ in water, azide group at the $\mathrm{N}$ terminus $\left(5^{\prime}\right)$, wild type of probe for colon cancer), and SPDP-PEG ${ }_{4}$-CTA CGC CAC CAG CT-NH ${ }_{2}$ for (protected) thiol-PNA (369 $\mu \mathrm{M}$ in water, SPDP $=N$-succinimidyl 3-(2-pyridyldithio)propionate, $P E G=$ poly(ethylene glycol), thiol group at the $\mathrm{N}$ terminus $\left(5^{\prime}\right)$, wild type of probe for colon cancer). The thiol-PNA was deprotected by adding $1 \mathrm{mM}$ TCEP in PBS. The used ( $n$ )CDNA sequences were obtained from Eurofins Genomics and included 5'-GCG TGC CAA CGC GCT GCG CAT-3' (100 $\mu \mathrm{M}$ in water) as CDNA for azido-PNA ${ }_{1}$ and 5'-AGC TGG TGG CGT AG-3' (100 $\mu \mathrm{M}$ in water) as CDNA for azido-PNA $A_{2}$ and thiol-PNA. The latter cDNA was obtained both with and without fluorescent rhodamine at its $5^{\prime}$ end. As dye-ncDNA, the sequence 5'-CTA CGC CAC CAG CT-3' was obtained with a rhodamine dye at the $5^{\prime}$ end.

\subsubsection{Methods}

Silicon oxide patterning. To make a pattern of silicon oxide dots, first a $160 \mathrm{~nm}$ thick $\mathrm{SiO}_{2}$ layer was grown by wet oxidation on a cleaned silicon $\mathrm{p}(100)$ wafer. A photoresist layer was spin coated on the front side (OiR 906-12, $6000 \mathrm{rpm}, 30 \mathrm{~s}$ ), baked at $95^{\circ} \mathrm{C}$ for $90 \mathrm{~s}$, patterned using standard photolithography ( $3 \mathrm{~s}$ UV exposure), immersed in resist developer (OPD 4262, $45 \mathrm{~s}$ ), and baked at $120^{\circ} \mathrm{C}$ for $10 \mathrm{~min}$. This resulted in a hexagonal array of resist dots with both a diameter and spacing of $100 \mu \mathrm{m}$, which was used as a mask to etch away the surrounding silicon oxide layer by $135 \mathrm{~s}$ immersion in an aqueous 
BHF solution. After resist removal by acetone rinsing, the resulting substrate contained silicon oxide dots surrounded by $\mathrm{H}$-terminated silicon. Without extra $1 \% \mathrm{HF}$ dip, a 1,8-nonadiyne monolayer was formed following the procedure described below.

Monolayer formation of 1,8-nonadiyne. To form a 1,8-nonadiyne monolayer on silicon substrates by hydrosilylation (Scheme 6.3b), the pure 1,8-nonadiyne solution was first degassed by four freeze-pump-thaw cycles. The silicon substrates, i.e., planar silicon pieces or silicon nanowires on chip, were cleaned by 5 min ultrasonication in acetone and for the chips an additional 25 min piranha cleaning $\left(95 \% \mathrm{H}_{2} \mathrm{SO}_{4}\right.$ and $33 \% \mathrm{H}_{2} \mathrm{O}_{2}$ mixed at $3: 1 \mathrm{v} / \mathrm{v})$. A hydrogen-terminated surface was created by $2 \mathrm{~min}$ and $4 \mathrm{~min}$ exposure to an aqueous $1 \% \mathrm{HF}$ solution to remove the native oxide, respectively. After rinsing in Milli- $\mathrm{Q}$ water and drying in a nitrogen stream, the substrates were immersed in the degassed 1,8-nonadiyne solution inside a nitrogen glovebox. A round-bottom reaction flask was equipped with a capillary as a nitrogen inlet and a reflux condenser. The hydrosilylation reaction was performed overnight under a low continuous nitrogen flow at $160{ }^{\circ} \mathrm{C}$. Afterwards the samples were cleaned by immersion in hexane, rinsing with dichloromethane, rinsing with ethanol, 5 min ultrasonication in dichloromethane to remove any physisorbed material, and subsequently dried in a stream of nitrogen.

Click chemistry with azide-functionalized dye, Au NPs, PNA, or TEG. Copper-catalyzed azide-alkyne cycloaddition (click chemistry, Scheme 6.3b, Scheme 6.5a) was used to couple the fluorescent dye azide-fluor 488, azide-functionalized gold NPs, azidefunctionalized $\mathrm{PNA}_{1}$, or azide-functionalized TEG onto a 1,8-nonadiyne monolayer. The substrate was overnight incubated with $25 \mu \mathrm{L}$ of the azide solution ( $2 \mathrm{mM}$ azide-fluor 488 in water, azide-functionalized gold NPs as received, $297 \mu \mathrm{M}$ azido-PNA ${ }_{1}$ in water, $2 \mathrm{mM}$ azide-TEG in water) and $25 \mu \mathrm{L}$ of the catalyst solution $\left(2 \mathrm{mM} \mathrm{Cu}(\mathrm{II}) \mathrm{SO}_{4} .5 \mathrm{H}_{2} \mathrm{O}, 80 \mathrm{mM}\right.$ $\mathrm{L}$-ascorbic acid in water (for the azide-dye, azido-PNA $A_{1}$, and azide-TEG click chemistry) or in DMSO (for the azide-NPs)) in a silicone isolator (Electron Microscopy Sciences). A glass slide on top was used to avoid solvent evaporation. Afterwards, the samples were sequentially rinsed with water, ethanol, immersed in acetone to remove the glue of the isolator, and sonicated in PBS with $0.05 \% \mathrm{v} / \mathrm{v}$ Tween-20 for $2 \mathrm{~min}$ (azido-PNA ) or 5 min (azide-dye, azide-Au NPs, and azide-TEG). After rinsing with a $0.05 \% \mathrm{w} / \mathrm{v}$ EDTA solution in water to remove any copper traces, the substrate was dried under nitrogen.

Thiol-yne chemistry with thiol-PNA. Thiol-yne chemistry (Scheme 6.5b) was used to couple thiol-PNA onto a 1,8-nonadiyne monolayer. The substrate was covered with a $10 \mu \mathrm{M}$ solution of thiol-PNA in PBS. The reaction was performed for $1 \mathrm{~h}$ under illumination by a $365 \mathrm{~nm}$ light source (4 W) at a $0.5 \mathrm{~cm}$ distance. Subsequently, the sample was sonicated in PBS for 1 min, rinsed with water, and dried under nitrogen. 
Microcontact printing of azido-PNA or thiol-PNA. Poly(dimethylsiloxane) (PDMS) stamps were prepared by casting the precursor poly(dimethylsiloxane) and curing agent (Sylgard 184, Dow Corning) at 10:1 volume ratio onto a silicon master. Air bubbles were removed by vacuum for $30 \mathrm{~min}$, and the stamps were cured overnight at $60{ }^{\circ} \mathrm{C}$. Before microcontact printing, the cut stamps ( $10 \mu \mathrm{m}$ lines and $5 \mu \mathrm{m}$ spacing for azido-PNA , and $5 \mu \mathrm{m}$ lines and $3 \mu \mathrm{m}$ spacing for thiol-PNA) were oxidized by oxygen plasma (power tuned at $40 \mathrm{~mA}$ ) for $30 \mathrm{~s}$. The stamp for click chemistry was inked with $75 \mu \mathrm{L}$ of azido-PNA 2 ( $272 \mu \mathrm{M}$ in water) and $25 \mu \mathrm{L}$ of catalyst solution ( $2 \mathrm{mM} \mathrm{Cu}(\mathrm{I})\left(\mathrm{CH}_{3} \mathrm{CN}\right)_{4} \mathrm{PF}_{6}$ and $2 \mathrm{mM}$ TBTA in $\mathrm{CH}_{3} \mathrm{CN} /$ ethanol, ratio $2: 1 \mathrm{v} / \mathrm{v}$ ) for $4 \mathrm{~min}$. After drying in a stream of nitrogen, the stamp was brought into conformal contact with the substrate for $2 \mathrm{~h}$. Subsequently, the printed substrate was rinsed with ethanol and water, and dried under nitrogen. For the thiol-yne reaction, the stamp was inked with $40 \mu \mathrm{L}$ of thiol-PNA ( $25 \mu \mathrm{M}$ in PBS) for 4 min. After drying the stamp under nitrogen, the stamp was brought into conformal contact with the substrate for $75 \mathrm{~min}$ under UV illumination (365 nm $(4 \mathrm{~W})$ at a $0.5 \mathrm{~cm}$ distance). Afterwards, the substrate was rinsed with PBS and water, and dried in a stream of nitrogen.

PNA-DNA hybridization. Hybridization with dye-(n)cDNA was performed by covering the PNA-monolayer-containing sample with a $2 \mu \mathrm{M}$ solution of dye- $(\mathrm{n}) \mathrm{CDNA}$ in buffer $(5 \times \mathrm{SSC}$, optionally with $0.2 \% \mathrm{w} / \mathrm{v}$ SDS). The reaction was performed for $2 \mathrm{~h}$ at room temperature under aluminum foil. Afterwards, the samples were sonicated in PBS with $0.05 \% \mathrm{v} / \mathrm{v}$ Tween-20 for 2 min, rinsed with water, and dried in a stream of nitrogen.

\subsubsection{Equipment}

Contact angle measurements. Static contact angles were measured with Milli-Q water on a Krüss G10 Contact Angle Measuring Instrument equipped with a CCD camera. Contact angle values were determined automatically by a drop shape analysis software. Contact angles were measured directly after the reaction and averaged over at least three drops.

Fluorescence microscopy. Fluorescence microscopy images were recorded in air on an Olympus inverted research microscope IX71 equipped with a mercury burner U-RFL-T as light source and a digital Olympus DP70 camera. To image the fluorescence of the azide-fluor 488 dye, blue excitation ( $490 \leq \lambda_{\text {ex }} \leq 510 \mathrm{~nm}$ ) and green emission $\left(520 \leq \lambda_{e m} \leq 550 \mathrm{~nm}\right.$ ) were filtered using a Chroma filter cube. For the rhodamine-labeled DNA sequences, green excitation $\left(510 \leq \lambda_{e x} \leq 550 \mathrm{~nm}\right)$ and red emission $\left(\lambda_{e m} \geq 590 \mathrm{~nm}\right)$ were filtered using an Olympus filter cube. Intensity profiles were obtained by a rectangular average over a part of the surface.

X-ray photoelectron spectroscopy. XPS measurements were performed on a Quantera SXM setup from Physical Electronics equipped with an Al Ka X-ray source (1486.6 eV). A 
take-off angle of $45^{\circ}$ was used, and collected spectra were calibrated on the C1s peak at $284.8 \mathrm{eV}$.

High-resolution scanning electron microscopy. HR-SEM images of nanowires on a chip were obtained with a Zeiss Merlin HR-SEM system with an InLens or ESB detector, operated at a typical acceleration voltages of $1.4 \mathrm{kV}$.

Quartz crystal microbalance with dissipation monitoring. QCM-D sensograms were recorded using a Q-Sense E4 module (Biolin Scientific) with two peristaltic pumps. Siliconcoated QCM sensors were cleaned by 5 min immersion in a piranha solution $\left(95 \% \mathrm{H}_{2} \mathrm{SO}_{4}\right.$ and $33 \% \mathrm{H}_{2} \mathrm{O}_{2}$ mixed at $3: 1 \mathrm{v} / \mathrm{v}$ ) and 5 min ultrasonication in acetone. To expose only the active sensor area to $1 \% \mathrm{HF}$, the remaining areas of the silicon QCM sensors were first protected by photoresist. The active area at the top side of the sensor was covered with a small suction cup, after which photoresist OiR 907-17 was spin coated three times (1000 rpm, $30 \mathrm{~s}$ ). After baking for $10 \mathrm{~min}$ at $120^{\circ} \mathrm{C}$, the entire back side was covered with photoresist using the same spin coating parameters. After a 3 min $1 \% \mathrm{HF}$ dip, the resist was removed by acetone rinsing, and the substrates were immediately modified with a monolayer of 1,8-nonadiyne and azido-PNA $A_{1}$ or thiol-PNA as described above. Afterwards, QCM-D measurements were started by sequentially recording a baseline in Milli-Q water and buffer (PBS for azido-PNA ${ }_{1}$ and $5 \times$ SSC with $0.2 \%$ w/v SDS for thiol-PNA) until stable. Hybridization was tested with $3 \mu \mathrm{M}$ cDNA (azido-PNA $A_{1}$ ) or $2 \mu \mathrm{M}$ cDNA (thiol-PNA) solutions in the same buffer. The flow rate was set at $100 \mu \mathrm{L} / \mathrm{min}$, and the temperature was kept at $22^{\circ} \mathrm{C}$. The sensograms were treated with a linear baseline correction to correct for a drift in the signal.

\subsection{References}

1. S. Lee, H. Huang and M. Zelen, Stat. Methods Med. Res., 2004, 13, 443-456.

2. M.G. Best, N. Sol, I. Kooi, J. Tannous, B.A. Westerman, F. Rustenburg, P. Schellen, H. Verschueren, E. Post, J. Koster, B. Ylstra, N. Ameziane, J. Dorsman, E.F. Smit, H.M. Verheul, D.P. Noske, J.C. Reijneveld, R.J.A. Nilsson, B.A. Tannous, P. Wesseling and T. Wurdinger, Cancer Cell, 2015, 28, 666-676.

3. J.H. Appel, H. Ren, M.L.Y. Sin, J.C. Liao and J. Chae, Analyst, 2016, 141, 652-660.

4. M. Mir, A. Homs and J. Samitier, Electrophoresis, 2009, 30, 3386-3397.

5. J.G.E. Gardeniers and A. van den Berg, Anal. Bioanal. Chem., 2004, 378, 1700-1703.

6. L. Scheres, J. ter Maat, M. Giesbers and H. Zuilhof, Small, 2010, 6, 642-650.

7. A. Calabretta, D. Wasserberg, G.A. Posthuma-Trumpie, V. Subramaniam, A. van Amerongen, R. Corradini, T. Tedeschi, S. Sforza, D.N. Reinhoudt, R. Marchelli, J. Huskens and P. Jonkheijm, Langmuir, 2011, 27, 1536-1542.

8. P.E. Nielsen and M. Egholm, Curr. Issues Mol. Biol., 1999, 1, 89-104.

9. F.P. Schwarz, S. Robinson and J.M. Butler, Nucleic Acids Res., 1999, 27, 4792-4800.

10. H. Park, A. Germini, S. Sforza, R. Corradini, R. Marchelli and W. Knoll, Biointerphases, 2007, 2, 80-88. 
11. W. Cai, J.R. Peck, D.W. van der Weide and R.J. Hamers, Biosens. Bioelectron., 2004, 19, $1013-$ 1019.

12. F. Wei, B. Sun, Y. Guo and X.S. Zhao, Biosens. Bioelectron., 2003, 18, 1157-1163.

13. P. Michaels, M.T. Alam, S. Ciampi, W. Rouesnel, S.G. Parker, M.H. Choudhury and J.J. Gooding, Chem. Commun., 2014, 50, 7878-7880.

14. Y.L. Bunimovich, Y.S. Shin, W.S. Yeo, M. Amori, G. Kwong and J.R. Heath, J. Am. Chem. Soc., 2006, 128, 16323-16331.

15. G.J. Zhang, J.H. Chua, R.E. Chee, A. Agarwal, S.M. Wong, K.D. Buddharaju and N. Balasubramanian, Biosens. Bioelectron., 2008, 23, 1701-1707.

16. S.Y. Chen, J.G. Bomer, W.G. van der Wiel, E.T. Carlen and A. van den Berg, ACS Nano, 2009, 3, 3485-3492.

17. O. Seitz, P.G. Fernandes, G.A. Mahmud, H.C. Wen, H.J. Stiegler, R.A. Chapman, E.M. Vogel and Y.J. Chabal, Langmuir, 2011, 27, 7337-7340.

18. M.N. Masood, S. Chen, E.T. Carlen and A. van den Berg, ACS Appl. Mater. Interfaces, 2010, 2, 3422-3428.

19. Q.Y. Sun, L.C.P.M. de Smet, B. van Lagen, M. Giesbers, P.C. Thune, J. van Engelenburg, F.A. de Wolf, H. Zuilhof and E.J.R. Sudholter, J. Am. Chem. Soc., 2005, 127, 2514-2523.

20. Y. Li and C.Z. Cai, Chem. - Asian J., 2011, 6, 2592-2605.

21. S. Ciampi, T. Böcking, K.A. Kilian, M. James, J.B. Harper and J.J. Gooding, Langmuir, 2007, 23, 9320-9329.

22. C. Wendeln, S. Rinnen, C. Schulz, H.F. Arlinghaus and B.J. Ravoo, Langmuir, 2010, 26, 1596615971.

23. N.S. Bhairamadgi, S. Gangarapu, M.A. Caipa Campos, J.M.J. Paulusse, C.J.M. van Rijn and H. Zuilhof, Langmuir, 2013, 29, 4535-4542.

24. N.K. Devaraj, G.P. Miller, W. Ebina, B. Kakaradov, J.P. Collman, E.T. Kool and C.E.D. Chidsey, J. Am. Chem. Soc., 2005, 127, 8600-8601.

25. S.Y. Lim, W.-y. Chung, H.K. Lee, M.S. Park and H.G. Park, Biochem. Biophys. Res. Commun., 2008, 376, 633-636.

26. J. Escorihuela, M.J. Banuls, R. Puchades and A. Maquieira, J. Mater. Chem. B, 2014, 2, 85108517.

27. D. Meziane, A. Barras, A. Kromka, J. Houdkova, R. Boukherroub and S. Szunerits, Anal. Chem., 2012, 84, 194-200.

28. L. Danos, R. Greef and T. Markvart, Thin Solid Films, 2008, 516, 7251-7255.

29. T.K. Mischki, R.L. Donkers, B.J. Eves, G.P. Lopinski and D.D.M. Wayner, Langmuir, 2006, 22, 8359-8365.

30. A. Cattani-Scholz, D. Pedone, M. Dubey, S. Neppl, B. Nickel, P. Feulner, J. Schwartz, G. Abstreiter and M. Tornow, ACS Nano, 2008, 2, 1653-1660.

31. C. Nicosia, J. Cabanas-Danés, P. Jonkheijm and J. Huskens, ChemBioChem, 2012, 13, 778-782.

32. E.G. Hvastkovs and D.A. Buttry, Langmuir, 2009, 25, 3839-3844.

33. L. Scheres, A. Arafat and H. Zuilhof, Langmuir, 2007, 23, 8343-8346.

34. Y.L. Bunimovich, G.L. Ge, K.C. Beverly, R.S. Ries, L. Hood and J.R. Heath, Langmuir, 2004, 20, 10630-10638.

35. I. Park, Z.Y. Li, A.P. Pisano and R.S. Williams, Nano Lett., 2007, 7, 3106-3111.

36. H.H. Liu, T.H. Lin and J.T. Sheu, ACS Appl. Mater. Interfaces, 2013, 5, 10048-10053.

37. T.R. Chan, R. Hilgraf, K.B. Sharpless and V.V. Fokin, Org. Lett., 2004, 6, 2853-2855. 


\section{Chapter 7}

\section{Electrochemistry of redox-active guest molecules at $\beta$-cyclodextrin-functionalized silicon electrodes}

Functionalization of silicon-based sensing devices with self-assembled receptor monolayers offers flexibility and specificity towards the requested analyte as well as the possibility of sensor reuse. As electrical sensor performance is determined by electron transfer, we functionalized $\mathrm{H}$-terminated silicon substrates with $\beta$-cyclodextrin $(\beta-C D)$ molecules to investigate the electronic coupling between these host monolayers and the substrate. A trivalent (one ferrocene and two adamantyl moieties), redox-active guest was bound to the $\beta-C D$ surface with a coverage of about $10^{-11} \mathrm{~mol} / \mathrm{cm}^{2}$ and an overall binding constant of $1.5 \times 10^{9} \mathrm{M}^{-1}$. This packing density of the host monolayers on silicon is lower than that for similar $\beta-C D$ monolayers on gold. The monolayers were comparable on lowly doped $\mathrm{p}$-type and highly doped $\mathrm{p}^{++}$substrates regarding their packing density and the extent of oxide formation. Nonetheless, the electron transfer was more favorable on $\mathrm{p}^{++}$substrates, as shown by the lower values of the peak splitting and peak widths in the cyclic voltammograms. These results show that the electron transfer rate on the host monolayers is not only determined by the composition of the monolayer, but also by the doping level of the substrate.

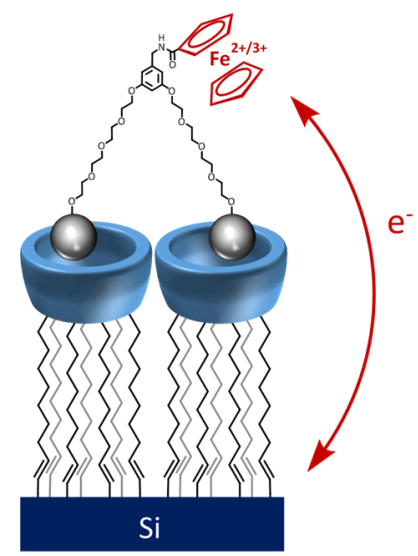

Part of this chapter has been published as: J. Veerbeek, A. Méndez-Ardoy and J. Huskens, ChemElectroChem, 2017, in press, DOI: 10.1002/celc.201600872. 


\subsection{Introduction}

Silicon substrates are of high interest for the fabrication of electronic devices, for example for sensing, ${ }^{1}$ data storage, ${ }^{2}$ and energy conversion. ${ }^{1}$ Placing functional groups onto the surface, for example by self-assembled monolayers, enables more flexibility and specificity towards an application, for instance to make an analyte-specific sensor. ${ }^{3-5}$ These devices, however, rely on direct charge transfer from/to the silicon substrate, which makes studies on these electron transfer processes essential. Typical monolayer parameters that influence the electron transfer include the way of surface coupling, the length and conductivity of the molecules used, and the packing of the monolayer.

When choosing molecules for self-assembled monolayers on sensing devices, supramolecular chemistry is attractive because of its modularity and reversibility. ${ }^{6,7}$ Specifically, cyclodextrin (CD) host molecules can be used for self-assembled monolayer formation by coupling to the surface. ${ }^{8-10} \mathrm{CD}$ molecules, which are cyclic oligosaccharides consisting of $6(\alpha), 7(\beta)$, or $8(\gamma) \alpha$-D-glucopyranose units, can be functionalized on their primary rim with substrate-coupling moieties, mostly performed in a multipodant way to increase monolayer stability. Monolayers of $\beta-C D$ have been applied, mostly for sensing purposes, on gold, ${ }^{11-18}$ glass, ${ }^{19-21}$ and silicon, ${ }^{22}$ for example.

For a silicon-based sensing device, oxide-free functionalization is required because any silicon oxide would function as an insulating layer and prevent charge transfer from/to the surface. ${ }^{23}$ Hydrosilylation is a suitable technique to functionalize silicon in an oxidefree way, since unsaturated carbon-carbon bonds are coupled to $\mathrm{H}$-terminated silicon, resulting in a direct $\mathrm{Si}-\mathrm{C}$ coupling. ${ }^{24,25} \mathrm{~A}$ few examples have been reported in which alkene-functionalized $\beta-C D$ molecules have been grafted onto $\mathrm{Si}-\mathrm{H}$ by a photochemical hydrosilylation method. ${ }^{22,26,27}$ These monolayers enabled measuring host-guest interactions by impedance spectroscopy ${ }^{22}$ or by a photoluminescence response, ${ }^{26,27}$ which confirmed the applicability of $\beta-C D$ host monolayers on silicon in sensing applications. These examples are based on electron transfer, which means that the sensor performance depends on the electronic coupling between the host-guest complexes and the substrate. The electron transfer processes between these $\beta$-CD host monolayers and the silicon substrate have, however, not been studied in detail.

Here, we use a redox-active guest to study the electronic coupling of a $\beta-C D$ host monolayer on silicon substrates. A new heptapodant alkyne-functionalized $\beta-C D$ molecule has been synthesized as the host molecule, since alkynes have been reported to result in better monolayers than alkenes. ${ }^{28,29}$ Monolayers of this $\beta$-CD derivative have been formed by a hydrosilylation reaction. Host-guest complexes were formed with a ferrocene-containing trivalent guest, since ferrocene-based guests have a characteristic redox signal. ${ }^{11,17,18}$ Electrochemistry was used to evaluate the properties of the electrical 
contact between the host layer and the underlying surface, as well as the influence of the doping concentration of the silicon substrates, i.e., lowly doped p-type (Si(p)) and highly doped $\mathrm{p}$-type $\left(\mathrm{Si}\left(\mathrm{p}^{++}\right)\right)$. Additionally, the electrochemical measurements were used to quantify the packing density of the host monolayer.

\subsection{Results and discussion}

Scheme 7.1a shows the monolayer formation on silicon substrates with heptaalkynefunctionalized $\beta-C D$ host 1 and its subsequent host-guest chemistry with the bisadamantyl ferrocene guest 2 . To avoid any insulating silicon oxide between the $\beta-C D$ monolayer and the substrate, functionalization of the Si surface with $\beta$-CD host molecules was achieved by hydrosilylation, by which unsaturated carbon-carbon bonds are coupled covalently to $\mathrm{H}$-terminated silicon. ${ }^{24,25}$ The heptaalkyne-functionalized $\beta$-CD molecule 1 (Scheme 7.1b) was synthesized by coupling 10-undecynoic acid to heptaaminofunctionalized $\beta-C D$. This resulted in complete substitution of all seven amino groups as confirmed by electrospray ionization mass spectrometry (ESI-MS). Formation of the desired product was further supported by elemental analysis and proton and carbon nuclear magnetic resonance (NMR) spectroscopy (see Figure 7.5 in the Experimental section).

a
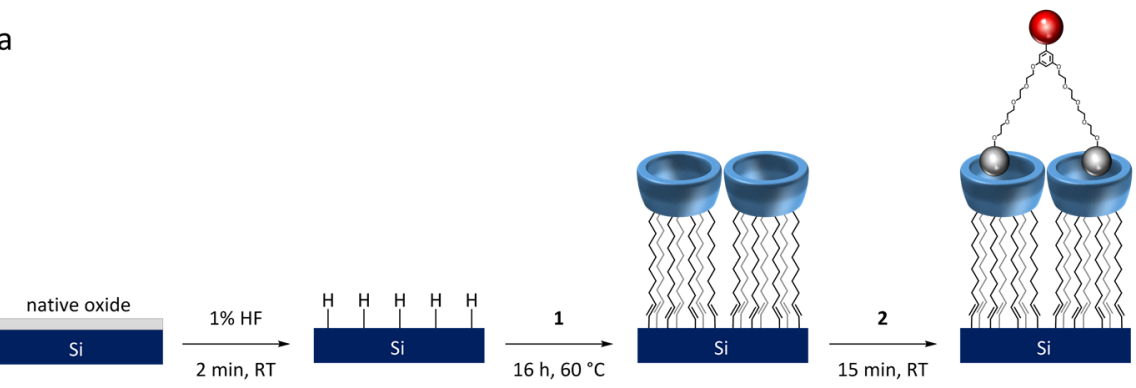

b
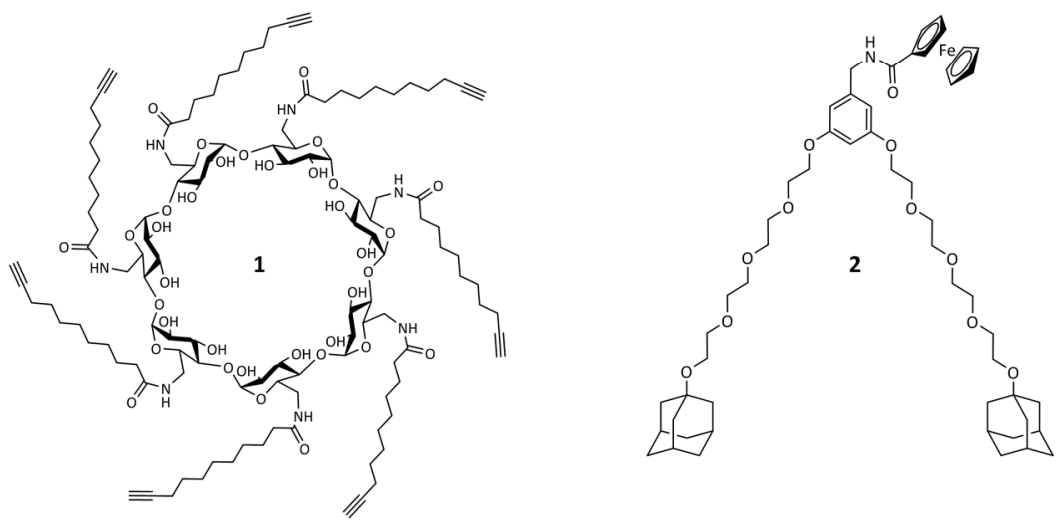

Scheme 7.1. a) Schematic procedure of silicon surface functionalization by covalent coupling of 1 to $\mathrm{H}$-terminated $\mathrm{Si}$, followed by host-guest chemistry with 2 . b) Chemical structures of heptaalkynefunctionalized $\beta-C D 1$ and bis-adamantyl ferrocene guest 2 . 


\subsubsection{Monolayer formation}

To make monolayers of 1 on $\mathrm{Si}(\mathrm{Si}-1)$, the native oxide layer of $\mathrm{Si}$ was first removed from lowly doped (p-type) or highly doped $\left(\mathrm{p}^{++}\right) \mathrm{Si}(100)$ substrates by short immersion in $1 \%$ aqueous hydrofluoric acid (HF). To enable electrochemistry at a later stage, an aluminum/silicon alloy was sputtered as Ohmic contact at the back side. The substrates were directly immersed in a $4 \mathrm{mM}$ solution of 1 in THF/DMF/mesitylene at $60^{\circ} \mathrm{C}$ for $16 \mathrm{~h}$. The resulting monolayer was characterized by contact angle measurements, showing $85.1^{\circ} \pm 1.9$ and $84.2^{\circ} \pm 3.3$ for $p$-type and $p^{++}$silicon, respectively. These values are higher than expected for the hydrophilic rim of the host cavity, as (advancing) contact angle values of $55^{\circ}$ and $49^{\circ}$ have been reported for $\beta-C D$ monolayers on gold ${ }^{30}$ and glass, ${ }^{8}$ respectively. Attenuated total reflection infrared spectroscopy (ATR-IR) spectra of dummy substrates, functionalized simultaneously with the electrochemistry samples, confirmed the formation of $\mathrm{Si}-1$ by the appearance of $\mathrm{O}-\mathrm{H} / \mathrm{N}-\mathrm{H}$ stretch vibrations in the $3600-3200 \mathrm{~cm}^{-1}$ range, $\mathrm{C}-\mathrm{H}$ stretches at 2968, 2937, $2857 \mathrm{~cm}^{-1}$ and the disappearance of $\mathrm{Si}-\mathrm{H}_{\mathrm{x}}$ stretches around $2108 \mathrm{~cm}^{-1}$ (Figure 7.1). Furthermore, no $\mathrm{C} \equiv \mathrm{CH}$ stretch vibration was visible at $3300 \mathrm{~cm}^{-1}$, thus indicating that the alkyne groups had fully reacted onto the surface.

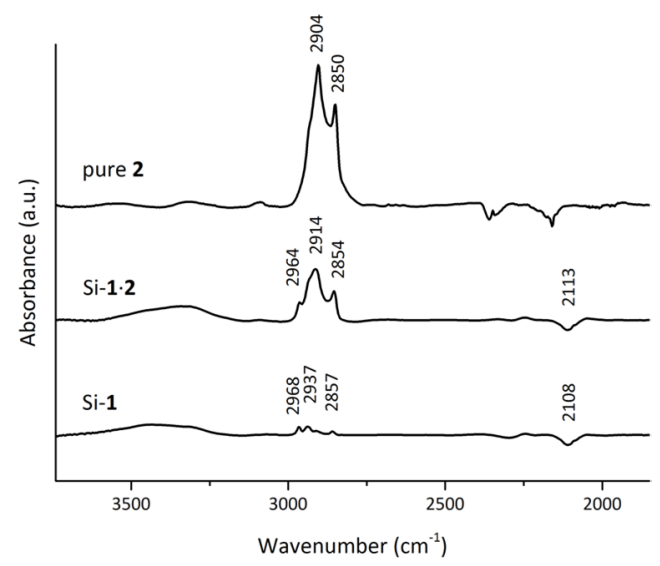

Figure 7.1. ATR-IR spectra of silicon substrates functionalized with 1 ( $\beta-C D, S i-1)$ and 2 ( $\beta-C D$ with guest 2, Si-1.2) referenced to an $\mathrm{H}$-terminated silicon sample. The FT-IR spectrum of pure 2 is added for comparison.

X-ray photoelectron spectroscopy (XPS) provided further evidence for successful Si-1 monolayer formation (Figure 7.2). The C1s core levels (Figure 7.2a, c) could be deconvoluted and fitted to three peaks at 288.3, 286.5, and $284.8 \mathrm{eV}$ for p-type Si and 288.5, 286.5, and $284.8 \mathrm{eV}$ for $\mathrm{p}^{++} \mathrm{Si}$, which correspond to the $\underline{\mathrm{C}}=\mathrm{O}, \underline{\mathrm{C}}-\mathrm{O} / \underline{\mathrm{C}}-\mathrm{N}$, and $\underline{\mathrm{C}}-\mathrm{C}$ moieties, respectively. ${ }^{31}$ The $\underline{\mathrm{C}}=\mathrm{O}$ peaks amounted to $6.7 \%$ (p-type) and $6.1 \%\left(\mathrm{p}^{++}\right)$of the total $C$ signal. These values match well with the expected value of $5.9 \%$. It was not possible to determine the number of alkyne groups bound to the surface, since the signals 
for neither the $\mathrm{Si}-\mathrm{C}=\mathrm{C}$ moiety $\left(283.5 \mathrm{eV}^{32}\right)$ nor the $\underline{\mathrm{C}}-\mathrm{C} \equiv \mathrm{C}$ moiety $\left(286.2 \mathrm{eV}^{32}\right)$ could be clearly deconvoluted in the $\mathrm{C} 1 \mathrm{~s}$ region. The atomic concentration ratios of $\mathrm{C}: \mathrm{N}$ equaled to 35 for both $\mathrm{p}$ and $\mathrm{p}^{++} \mathrm{Si}$, which is higher than the stoichiometric value of 17 , probably due to some adventitious carbon contaminants. The Si2p core spectrum (Figure 7.2b, d) shows that the surface (after storage under nitrogen for two days before measuring) was partly converted into $\mathrm{SiO}_{2}$, seen from the signals at $102.5 \mathrm{eV}$ (p-type, 14\%) or $102.4 \mathrm{eV}$ $\left(\mathrm{p}^{++}, 13 \%\right)$. This could be explained by $\mathrm{i}$ ) the bulkiness of the heptaalkyne-functionalized $\beta-C D$ molecules, resulting in a less densely packed monolayer, and/or ii) the covalent nature of the binding of the heptapodant host molecules, which lowers the lateral mobility compared to monolayer formation on gold. Any Si-O-C moieties would have been visible in the XPS spectra at lower binding energies, thus indicating that the $\beta-C D$ molecules were covalently coupled by $\mathrm{Si}-\mathrm{C}$ bonds, indicating that $\mathrm{SiO}_{2}$ only occurred at the nonreacted sites.
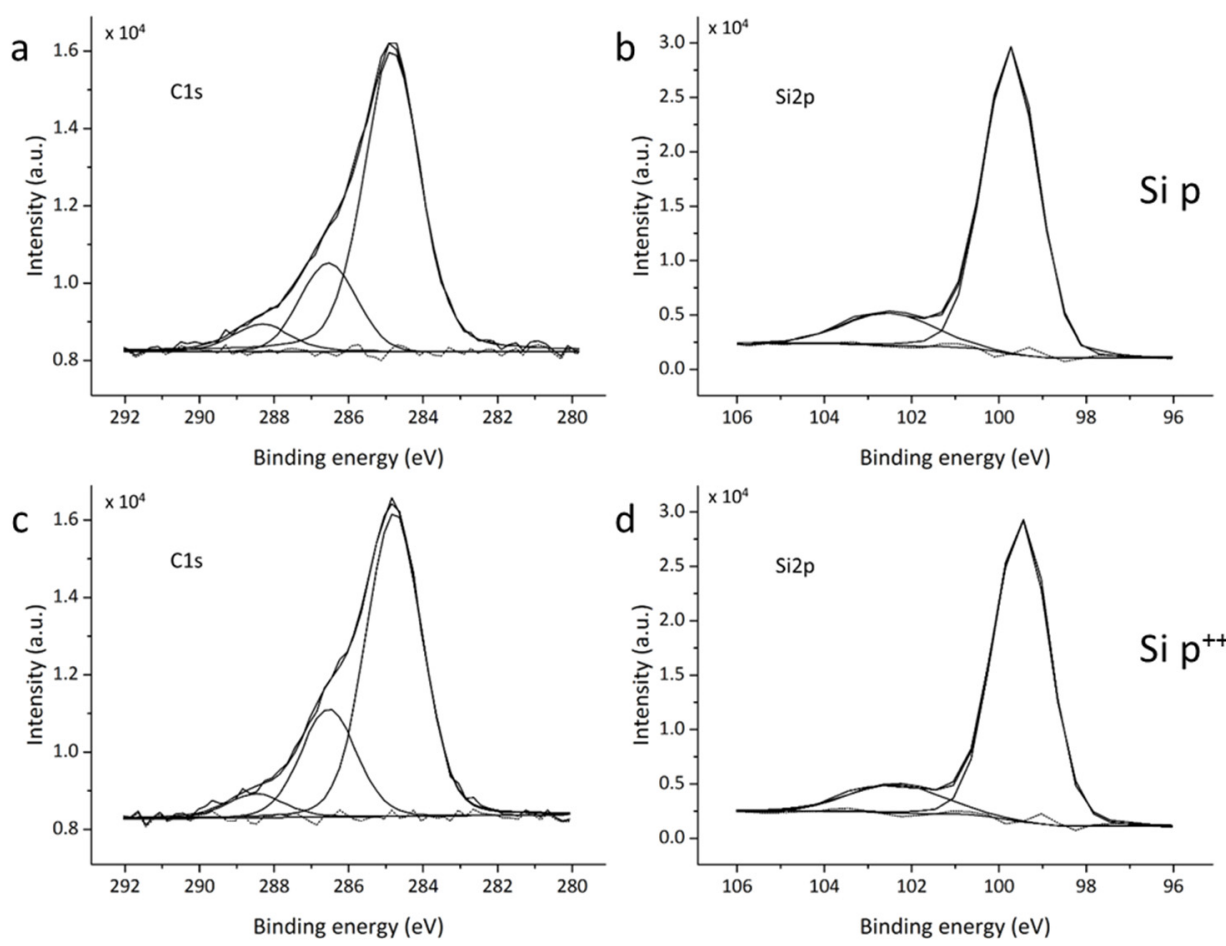

Figure 7.2. XPS spectra of Si-1 on a,b) p-type and c,d) $p^{++}$silicon, showing a,c) the $\mathrm{C} 1 \mathrm{~s}$ region and $b, d)$ the Si2p region including deconvoluted signals.

\subsubsection{Guest immobilization}

To study host-guest chemistry on Si-1, trivalent guest 2 (Scheme 7.1b) was used as designed before, ${ }^{11}$ consisting of two tetra(ethylene glycol) spacers with adamantyl moieties for stable anchoring onto $\beta-C D$ surfaces and an electroactive ferrocene moiety 
linked through a benzene ring. Guest immobilization was achieved by immersion in a $10 \mu \mathrm{M}$ solution of 2 , resulting in silicon surfaces with covalently bound $\beta-C D$ and supramolecularly bound ferrocene guest (Si-1-2). The concentration of $10 \mu \mathrm{M}$ is expected to lead to practically saturated, divalently bound 2 on the surface of Si-1. ${ }^{11}$ The contact angle of these surfaces was $83.7^{\circ} \pm 2.0$ and $79.5^{\circ} \pm 1.2$ for $p$-type and $\mathrm{p}^{++}$silicon, respectively, which is slightly more hydrophilic than the Si-1 host surfaces. ATR-IR (Figure 7.1) showed a clear increase in the $\mathrm{C}-\mathrm{H}$ stretch signals. XPS of Si-1.2 surfaces further confirmed guest immobilization by the presence of Fe (data not shown).

Electrochemical experiments were used to study the electronic coupling between the guest 2 and the Si-1 surface and the density of $\beta-C D$ groups on the surface. After guest immobilization, cyclic voltammograms were measured without guest or $\beta-C D$ in solution (Figure 7.3a, d). The ferrocene redox activity is clearly visible, with a half-wave potential $\left(E_{1 / 2}\right)$ of 0.523 and $0.528 \mathrm{~V}$ vs. $\mathrm{Ag} / \mathrm{AgCl}$ for $\mathrm{p}$ and $\mathrm{p}^{++}$silicon, respectively, which is comparable to previous measurements on gold. ${ }^{11}$ Cyclic voltammetry showed that the host-guest binding was reversible, since the ferrocene peaks disappeared upon competition with an aqueous $10 \mathrm{mM} \beta-\mathrm{CD}$ solution (Figure 7.3c, f). The peak current increased linearly with the scan rate (Figure $7.3 \mathrm{~b}, \mathrm{e}$ ), which confirms that the redox activity originates from a surface-confined species instead of a diffusion-controlled reaction. ${ }^{33}$ The slope of these graphs can be used to calculate the surface coverage of the guest molecules by Equation (1):

$$
i_{p}=\frac{n^{2} F^{2}}{4 R T} A \Gamma_{0} v
$$

where $i_{p}$ is the peak current, $n$ is the number of electrons transferred in the redox event, $F$ is the Faraday constant, $R$ is the gas constant, $T$ is the temperature, $A$ is the electrode area, $\Gamma_{0}$ is the surface coverage, and $v$ is the scan rate. Using the graph of the anodic peak current versus the scan rate, guest surface coverages of $1.2 \times 10^{-11} \mathrm{~mol} / \mathrm{cm}^{2}$ and $1.1 \times 10^{-11} \mathrm{~mol} / \mathrm{cm}^{2}$ were found for $\mathrm{p}$ and $\mathrm{p}^{++}$substrates, respectively. Assuming divalent guest binding as reported before, ${ }^{11}$ the host coverages equaled to $2.4 \times 10^{-11} \mathrm{~mol} / \mathrm{cm}^{2}$ and $2.2 \times 10^{-11} \mathrm{~mol} / \mathrm{cm}^{2}$, respectively. The packing density of $\beta-C D$ monolayers on silicon has not been reported before, but alkyl-functionalized $\beta-C D$ molecules on gold showed a host coverage of $5.9 \times 10^{-11} \mathrm{~mol} / \mathrm{cm}^{2}$ determined by the same method. ${ }^{11}$ It can be concluded that the $\beta-C D$ monolayers on silicon showed about half of the packing densities compared to the highly packed monolayers on gold. This is in line with the XPS data that showed the presence of $\mathrm{SiO}_{2}$. 

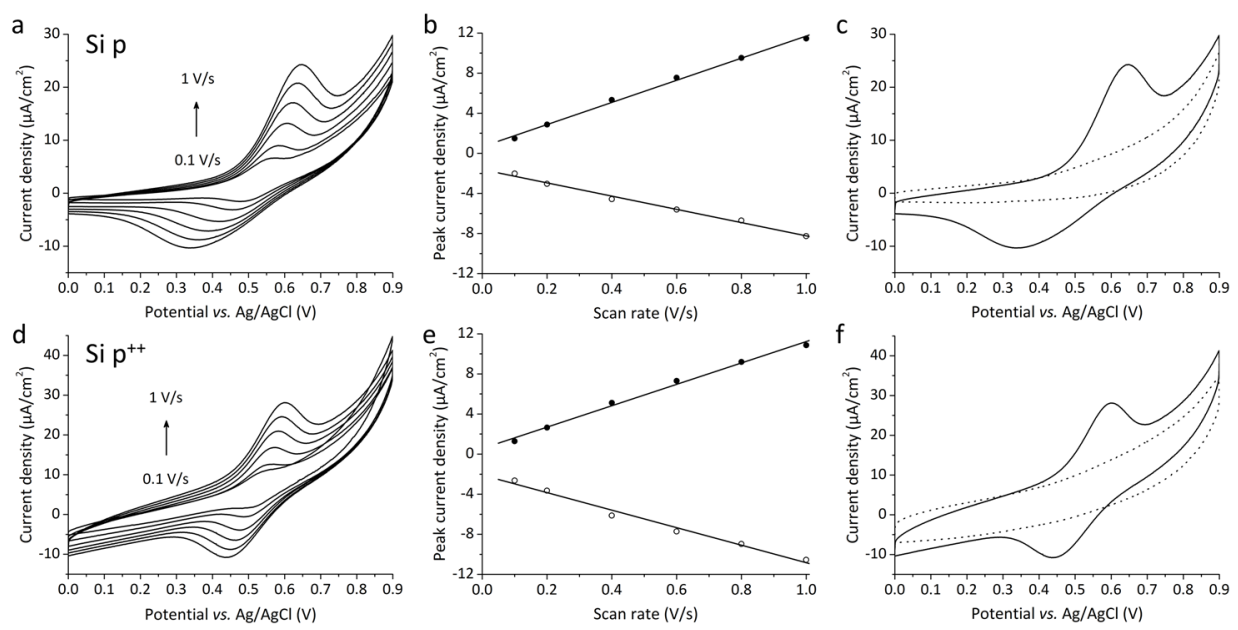

Figure 7.3. Electrochemical characterization of Si-1.2 on a-c) p-type and d-f) $\mathrm{p}^{++}$substrates. a,d) Cyclic voltammetry at $0.1,0.2,0.4,0.6,0.8$ and $1.0 \mathrm{~V} / \mathrm{s}$ in an aqueous solution of $1 \mathrm{M} \mathrm{NaClO}_{4}$. $b, e)$ The corresponding graphs reporting the dependence of the anodic (closed dots) and cathodic (open dots) peak current densities on scan rate. $\mathrm{c}, \mathrm{f}$ ) Cyclic voltammograms at $1 \mathrm{~V} / \mathrm{s}$ before (solid line) and after (dotted line) competition with a $10 \mathrm{mM} \beta-C D$ solution in water.

Whereas the host-guest chemistry described so far was comparable for $\mathrm{p}$ and $\mathrm{p}^{++} \mathrm{Si}$, the electron transfer properties slightly differed. The peak splitting at $0.1 \mathrm{~V} / \mathrm{s}$ was $52 \mathrm{mV}$ for $\mathrm{p}$-type $\mathrm{Si}$ and $18 \mathrm{mV}$ for $\mathrm{p}^{++} \mathrm{Si}$, of which the latter value is the closest to the expected value of $0 \mathrm{mV}$ for an ideal surface-confined electron transfer process. The peak separation increased for higher scan rates, e.g., to $286 \mathrm{mV}$ for $\mathrm{p}$-type Si and $136 \mathrm{mV}$ for $\mathrm{p}^{++}$Si at $1 \mathrm{~V} / \mathrm{s}$, which indicates that the electron transfer gets kinetically limited and shifts from reversible to quasi-reversible. This effect was confirmed by unequal peak currents for the anodic and cathodic reactions. Irreversible electron transfer (peak splitting $>200 \mathrm{mV}^{34}$ ) was observed upward of $0.8 \mathrm{~V} / \mathrm{s}$ for $\mathrm{p}$-type $\mathrm{Si}$ and $2 \mathrm{~V} / \mathrm{s}$ for $\mathrm{p}^{++} \mathrm{Si}$. This behavior could be due to the linkers in the host and guest molecules used, which place the ferrocene moiety relatively far from the Si surface and thus retard the electron transfer. ${ }^{11}$ Alternatively, the electron transfer could be retarded by the presence of $\mathrm{SiO}_{2}$ due to the non-close-packed $\beta-C D$ monolayer.

Full width at half maximum (FWHM) values of the cyclic voltammetry peaks were determined to be 98 and $91 \mathrm{mV}$ (anodic peak, $0.1 \mathrm{~V} / \mathrm{s}$ ) for $\mathrm{p}$ and $\mathrm{p}^{++}$substrates, respectively. These values are just slightly larger than or equal to the theoretical value of $91 \mathrm{mV} .{ }^{35}$ Nonetheless, the FWHM values were not independent of the scan rate as they should be, since the values at $1.0 \mathrm{~V} / \mathrm{s}$ increased to 130 (p-type) and $118 \mathrm{mV}\left(\mathrm{p}^{++} \mathrm{Si}\right.$ ). The peak splitting and FWHM values of silicon $\mathrm{p}^{++}$substrates were comparable to monolayers of heptathioether $\beta-C D$ with the same guest on gold substrates. ${ }^{11}$ Also for this monolayer, the alkyl chains place the host cavity relatively far from the substrate, which is further 
enhanced by the linker in the guest molecule. Therefore, the retarded electron transfer in the Si-1.2 system is taken as a sign of the occurrence of an insulating alkyl layer between the host cavity and the surface.

In short, the host monolayers on $\mathrm{p}$ and $\mathrm{p}^{++}$substrates were chemically equivalent, as both surfaces showed similar coverages and $\mathrm{SiO}_{2}$ percentages. Nonetheless, the electron transfer properties were more favorable for the $\mathrm{p}^{++}$substrates, as seen from the lower peak splitting and peak widths. Thus, the electron transfer rate is not only limited by the thickness and coupling of the $\beta-C D$ monolayer but is also determined by the doping level of the substrate. The better electrochemical response on the $\mathrm{p}^{++}$substrates is expected to originate from their higher conductivity, which enables a better charge transfer from the host-guest complexes to the substrate.

To further investigate the host-guest chemistry, the effect of the variation of the guest concentration was monitored by electrochemistry, as performed on $\mathrm{p}^{++} \mathrm{Si}-1$ by measuring the coverage of guest 2 by cyclic voltammetry with guest 2 in solution. Starting from a bare host surface and with guest molecules in an aqueous solution complexed with free $\beta-C D$, an equilibrium should favor the binding of the guest to the $\beta-C D$ monolayer. The surface coverage was determined for several guest concentrations ranging from 0.1 to $8.0 \mu \mathrm{M}$ in the presence of $1 \mathrm{mM} \beta-\mathrm{CD}$ as competitor and $1 \mathrm{M} \mathrm{NaClO}_{4}$ as electrolyte. Figure 7.4a shows that the shape of a plot of surface coverage vs. guest 2 concentration is comparable to an SPR adsorption isotherm on gold surfaces with a $\beta$-CD monolayer. ${ }^{36}$ Each of the data series shows a linear scan rate dependence (Figure 7.4b), which indicates that the major part measured is surface-confined, even though there is guest present in solution. The increase of the intercepts with the $y$-axis $>0$ at higher concentrations indicates, however, a minor contribution of solution species for these series. Fitting with a combined diffusion-controlled and surface-confined model to determine the surface coverage $^{33}$ did not yield a better fit, thus we found the diffusion part negligible. As another control, the $8.0 \mu \mathrm{M}$ guest solution was also measured on top of a bare $\mathrm{Si}$ substrate, which instead gave a linear dependence on the square root of the scan rate (Figure 7.4c). The peak splitting was smaller when measuring this diffusion-controlled species on a bare Si substrate compared to the surface-confined species on a Si-1 substrate (Figure 7.4d), which made a clear distinction between diffusion-controlled and surface-confined peaks possible. Consequently, the redox-active species as measured in Figure 7.4a and b were assumed to be surface-confined. 

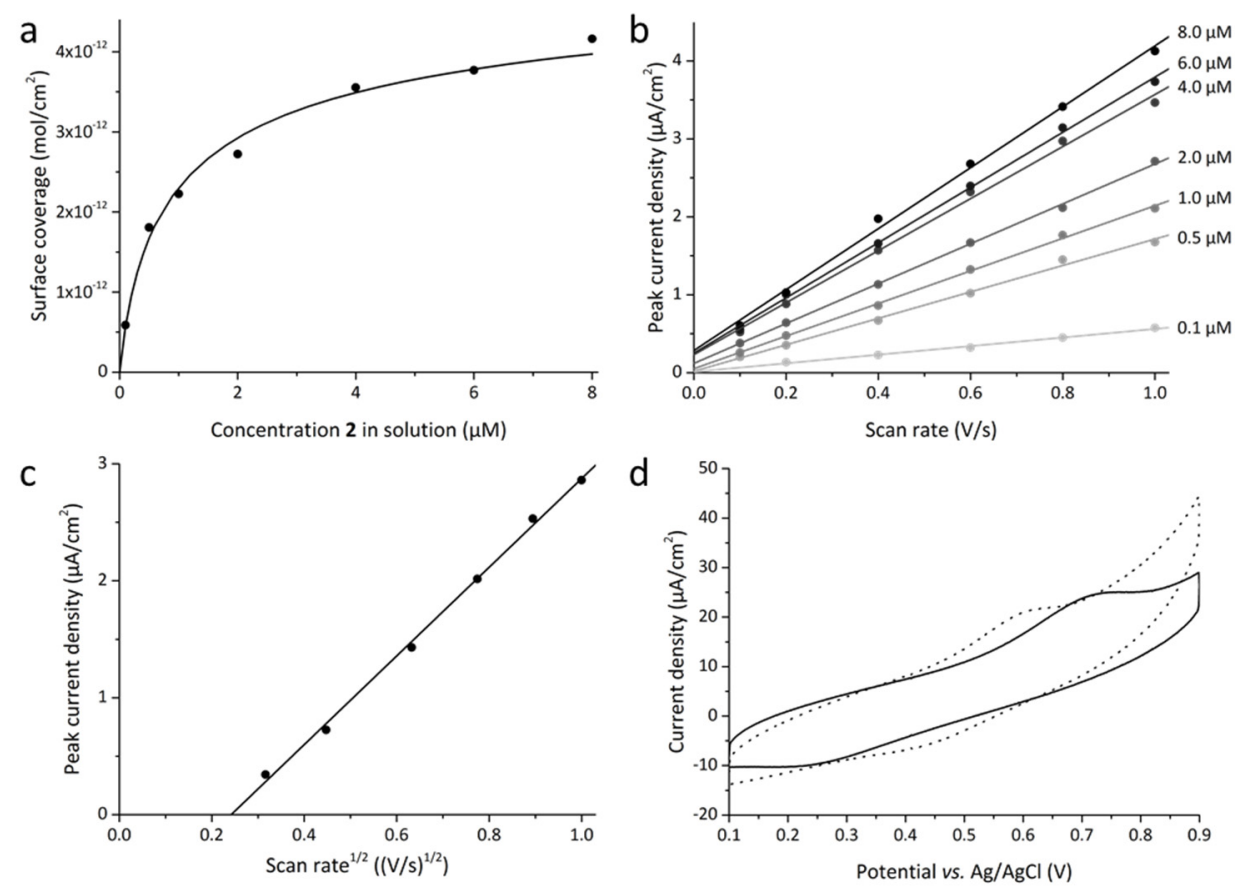

Figure 7.4. a) Titration of guest 2 in an aqueous $1 \mathrm{mM} \beta-\mathrm{CD}$ solution with $1 \mathrm{M} \mathrm{NaClO}_{4}$ on $\mathrm{Si}\left(\mathrm{p}^{++}\right)-1$, where each surface coverage was determined by cyclic voltammetry at different scan rates and the solid line is a fit to a thermodynamic multivalent model. b) The corresponding graphs on scan rate dependence of the anodic peak current density for each surface coverage determination (the data point at $0.1 \mathrm{~V} / \mathrm{s}$ for $0.1 \mu \mathrm{M}$ guest was omitted because the peak current was too low to be determined). c) The linear dependence of the peak current density versus the square root of the scan rate for cyclic voltammetry of $8.0 \mu \mathrm{M}$ guest 2 in solution on a bare silicon substrate. d) Cyclic voltammetry at $1 \mathrm{~V} / \mathrm{s}$ with $8.0 \mu \mathrm{M}$ of guest 2 in solution ( $1 \mathrm{mM} \beta-\mathrm{CD}$ and $1 \mathrm{M} \mathrm{NaClO}_{4}$ in water) on $\mathrm{p}^{++}$Si functionalized with 1 (solid line, peak positions 0.260 and $0.705 \mathrm{~V}$ ) and bare $\mathrm{p}^{++} \mathrm{Si}$ (dotted line, peak positions 0.453 and $0.589 \mathrm{~V}$ ).

The host-guest interactions were further quantified by fitting the data using a thermodynamic model for multivalent host-guest interactions on a surface in the presence of a competing guest in solution. ${ }^{11,37}$ The fitting was performed using $K_{i, s}$ and the maximum surface coverage as variables and $K_{i, l}=4.6 \times 10^{4} \mathrm{M}^{-1}, C_{\text {eff }}=0.2 \mathrm{M}$, and $\left[\beta-C D_{l}\right]=1 \mathrm{mM}$ as fixed parameters. ${ }^{36}$ Assuming divalent guest binding through both adamantyl moieties as reported before for the same guest, ${ }^{11}$ the fitting resulted in $K_{i, s}=8.6 \times 10^{4} \mathrm{M}^{-1}$ and a maximum guest coverage of $1.1 \times 10^{-11} \mathrm{~mol} / \mathrm{cm}^{2}$, i.e., an overall observed binding constant $\left(K_{\text {obs }}=K_{i, s}{ }^{2} \times C_{\text {eff }}\right)$ of $1.5 \times 10^{9} \mathrm{M}^{-1}$. The $K_{i, s}$ value is slightly lower than observed before for $\beta$-CD monolayers on gold with another divalent adamantane guest $\left(1.6 \times 10^{5} \mathrm{M}^{-1}\right)$, ${ }^{36}$ which could be due to the lower packing density observed. The saturation guest coverage is comparable to the guest-incubated samples described above without host or guest molecules in solution $\left(1 \times 10^{-11} \mathrm{~mol} / \mathrm{cm}^{2}\right)$. 


\subsection{Conclusions}

Overall, we have studied host-guest chemistry on silicon $\mathrm{p}$ and $\mathrm{p}^{++}$surfaces functionalized with monolayers of $\beta-C D$. Supramolecular interactions with a trivalent ferrocenefunctionalized guest were shown to be reversible by competition with free $\beta-C D$ in solution. The host coverages were equal to $2 \times 10^{-11} \mathrm{~mol} / \mathrm{cm}^{2}$, which indicated that the $\beta-C D$ monolayers on silicon have a coverage of about half of the values reported for comparable $\beta-C D$ monolayers on gold. This could be explained by the bulkiness of the heptaalkyne-functionalized $\beta-C D$ molecules and/or the covalent nature of the binding of the heptapodant host molecules, which prohibits the lateral mobility during monolayer formation. A titration was performed at varying concentrations of guest while also using free $\beta$-cyclodextrin in solution as a competitor, by monitoring the cyclic voltammetry signal. These results showed an overall observed binding constant of the guest on a $\beta$-cyclodextrin-functionalized silicon $\mathrm{p}^{++}$substrate of $1.5 \times 10^{9} \mathrm{M}^{-1}$. The electron transfer between the host-guest complex and the substrate was more kinetically limited at $p$-type substrates than on $\mathrm{p}^{++}$surfaces, as indicated by a larger peak splitting in the cyclic voltammograms and larger peak widths. Even though the insulating alkyl layer between the $\beta-C D$ and the silicon substrates is expected to retard the electron transfer, the monolayers were chemically equivalent on both substrate types and could not explain the differences observed in cyclic voltammetry. The doping level of the substrate thus also influenced the electrochemical response on the host layer. The electrochemical response could be measured better on the $\mathrm{p}^{++}$substrates, which indicates that these substrates are the preferred candidates for silicon-based sensors over lowly doped p-type silicon. Further research is required to elucidate the stability of the electrodes in aqueous environments, as prolonged cyclic voltammetry could lead to wave broadening and electrode oxidation, for example. ${ }^{38-40}$ The electrochemical response and stability may be improved further by backfilling the host monolayer with spectator molecules, which avoids oxidation of the surface after $\beta-C D$ monolayer formation. ${ }^{41}$

\subsection{Acknowledgments}

Alejandro Méndez-Ardoy is gratefully acknowledged for the syntheses of heptaalkynefunctionalized $\beta-C D 1$ and ferrocene-containing trivalent guest 2 .

\subsection{Experimental section}

\subsubsection{Materials}

Silicon wafers (<100>-oriented, $100 \mathrm{~mm}$ diameter, single side polished) were obtained from Okmetic (Finland) as p-type (boron, resistivity 5-10 $\Omega \cdot \mathrm{cm}$ ) or $\mathrm{p}^{++}$(boron, 0.010-0.025 $\Omega \cdot \mathrm{cm}$ ). For infrared spectroscopy, double-sided $p$-type silicon wafers were used with parameters equal to the single side polished p-type wafers. Reagents and 
solvents were obtained from commercial sources and used without further purification unless stated otherwise. Mesitylene (>98\%), dichloromethane (99.7\%), and $\mathrm{N}, \mathrm{N}$-dimethylformamide (DMF, 99.8\%) were dried over molecular sieves $(0.3 \mathrm{~nm})$. Tetrahydrofuran (THF) was obtained from a solvent purification system (MB SPS-800). The trivalent guest 2 was synthesized as described before. ${ }^{11}$ Milli-Q water with a resistivity $>18 \mathrm{M} \Omega \cdot \mathrm{cm}$ was obtained from a Milli-Q Integral water purification system (Merck Millipore). Glassware used for the hydrosilylation reactions was dried overnight at $120^{\circ} \mathrm{C}$.

\subsubsection{Methods}

Synthesis of heptakis-6-deoxy-6-(undec-10-ynamido)cyclomaltoheptaose 1. Heptakis(6amino-6-deoxy)cyclomaltoheptaose was prepared following the procedure of Ashton et al. ${ }^{42}$ and further purified by repeated precipitation of the aqueous solution of the hydrochloride by adding aqueous solution of ammonium hydroxide (30\%) until reaching the $\mathrm{pH}$ 9-10. The precipitate was then filtered off, washed with water, methanol, and diethyl ether and dried in vacuum. A solution of 10-undecynoic acid (339 mg, $1.89 \mathrm{mmol}$, $1 \mathrm{eq}$ ) and 1-hydroxybenzotriazole (287 mg, $2.08 \mathrm{mmol}, 1.1 \mathrm{eq})$ in dry DMF (13 mL) was cooled down to $0{ }^{\circ} \mathrm{C}$, and $\mathrm{N}$, $\mathrm{N}^{\prime}$-dicyclohexylcarbodiimide (385 mg, $1.89 \mathrm{mmol}, 1 \mathrm{eq}$ ) was added. The mixture was stirred under argon for $1 \mathrm{~h}$ at $0{ }^{\circ} \mathrm{C}$ and $1 \mathrm{~h}$ at room temperature. A slurry of heptakis(6-amino-6-deoxy)cyclomaltoheptaose $(300 \mathrm{mg}, 0.27 \mathrm{mmol}$ ) and $N$-ethylmorpholine $(0.2 \mathrm{~mL}, 1.89 \mathrm{mmol}, 1 \mathrm{eq})$ in $\mathrm{DMF}(13 \mathrm{~mL})$ was added, and the reaction mixture was stirred for 3 days. DMF was evaporated to about $10 \mathrm{~mL}$ and added to $80 \mathrm{~mL}$ water to form a suspension. The solid was filtered off and washed with water $(3 \times 10 \mathrm{~mL})$ and diethyl ether $(3 \times 10 \mathrm{~mL})$. The solid was purified by silica gel column chromatography eluting with 9:1 $\rightarrow$ 5:1 dichloromethane-methanol to give compound 1 as an off-white syrup. Yield $108 \mathrm{mg}(18 \%) . R_{f}=0.42$ (5:1 dichloromethane-methanol). ${ }^{1} \mathrm{H} \mathrm{NMR}\left(400 \mathrm{MHz}\right.$, methanol- $d_{4}$, Figure 7.5) $\delta 8.26\left(\mathrm{t}, 7 \mathrm{H}, \mathrm{J}_{\mathrm{NH}, \mathrm{CH}}=5.3 \mathrm{~Hz}, \mathrm{NH}\right), 4.97(\mathrm{~d}, 7$ $\left.\mathrm{H}, J_{1,2}=3.5 \mathrm{~Hz}, \mathrm{H}-1\right), 3.98\left(\mathrm{bd}, 7 \mathrm{H}, J_{6 a, 6 b}=12.6 \mathrm{~Hz}, \mathrm{H}-6 \mathrm{a}\right), 3.86\left(\mathrm{t}, 7 \mathrm{H}, J_{2,3}=J_{3,4}=9.2 \mathrm{~Hz}, \mathrm{H}-\right.$ 3), $3.86(\mathrm{~m}, 7 \mathrm{H}, \mathrm{H}-5), 3.54(\mathrm{dd}, 7 \mathrm{H}, \mathrm{H}-2), 3.46(\mathrm{~m}, 7 \mathrm{H}, \mathrm{H}-6 \mathrm{~b}), 3.32\left(\mathrm{t}, 7 \mathrm{H}, J_{4,5}=9.3 \mathrm{~Hz}, \mathrm{H}-\right.$ 4), $2.31\left(\mathrm{t}, 14 \mathrm{H},{ }^{3} \mathrm{~J}_{\mathrm{H}, \mathrm{H}}=7.3 \mathrm{~Hz}, \mathrm{CH}_{2} \mathrm{CO}\right), 2.21\left(\mathrm{~m}, 21 \mathrm{H}, \equiv \mathrm{CH}, \mathrm{CH}_{2} \mathrm{C} \equiv \mathrm{CH}\right), 1.66$ (bs, $14 \mathrm{H}$, $\left.\mathrm{COCH}_{2} \mathrm{CH}_{2}\right), 1.52\left(\mathrm{q}, 14 \mathrm{H},{ }^{3} \mathrm{~J}_{\mathrm{H}, \mathrm{H}}=6.6 \mathrm{~Hz}, \mathrm{CH}_{2} \mathrm{CH}_{2} \mathrm{C} \equiv \mathrm{CH}\right), 1.5-1.3\left(\mathrm{~m}, 56 \mathrm{H}, \mathrm{COCH}_{2} \mathrm{CH}_{2}\left(\mathrm{CH}_{2}\right)_{4}\right)$. ${ }^{13} \mathrm{C}$ NMR (100.3 MHz, methanol- $d_{4}$, Figure 7.5) $\delta 176.3$ (CO), 104.0 (C-1), 85.5 (C-4), 85.1 $(\mathrm{C} \equiv \mathrm{CH}), 74.5,74.2,72.4(\mathrm{C}-5, \mathrm{C}-2, \mathrm{C}-3), 70.0(\mathrm{C} \equiv \mathrm{CH}), 41.3(\mathrm{C}-6), 37.1\left(\mathrm{COCH}_{2}\right), 30.4-29.7$ $\left(\mathrm{COCH}_{2} \mathrm{CH}_{2}\left(\mathrm{CH}_{2}\right)_{5}\right), 27.2\left(\mathrm{COCH}_{2} \mathrm{CH}_{2}\right), 19.1\left(\mathrm{CH}_{2} \mathrm{C} \equiv \mathrm{CH}\right)$. ESI-MS $\mathrm{m} / \mathrm{z}$ calculated 1139.91, found $1139.50[\mathrm{M}+2 \mathrm{H}]^{2+}$ and calculated 2278.81, found $2278.34[\mathrm{M}+\mathrm{H}]^{+}$. Anal. calcd. for $\mathrm{C}_{119} \mathrm{H}_{189} \mathrm{~N}_{7} \mathrm{O}_{35} \cdot 2 \mathrm{H}_{2} \mathrm{O}$ C 61.77, H 8.41, N 4.24, found C 61.84, H 8.01, N 3.80. 


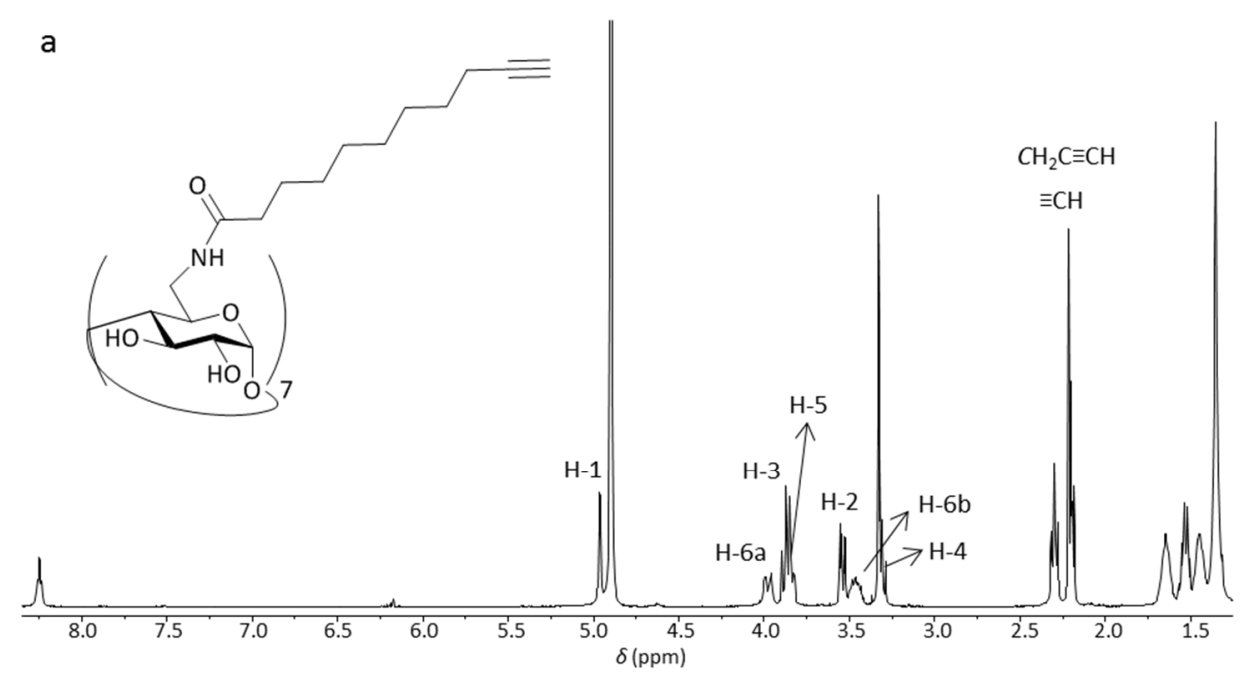

b

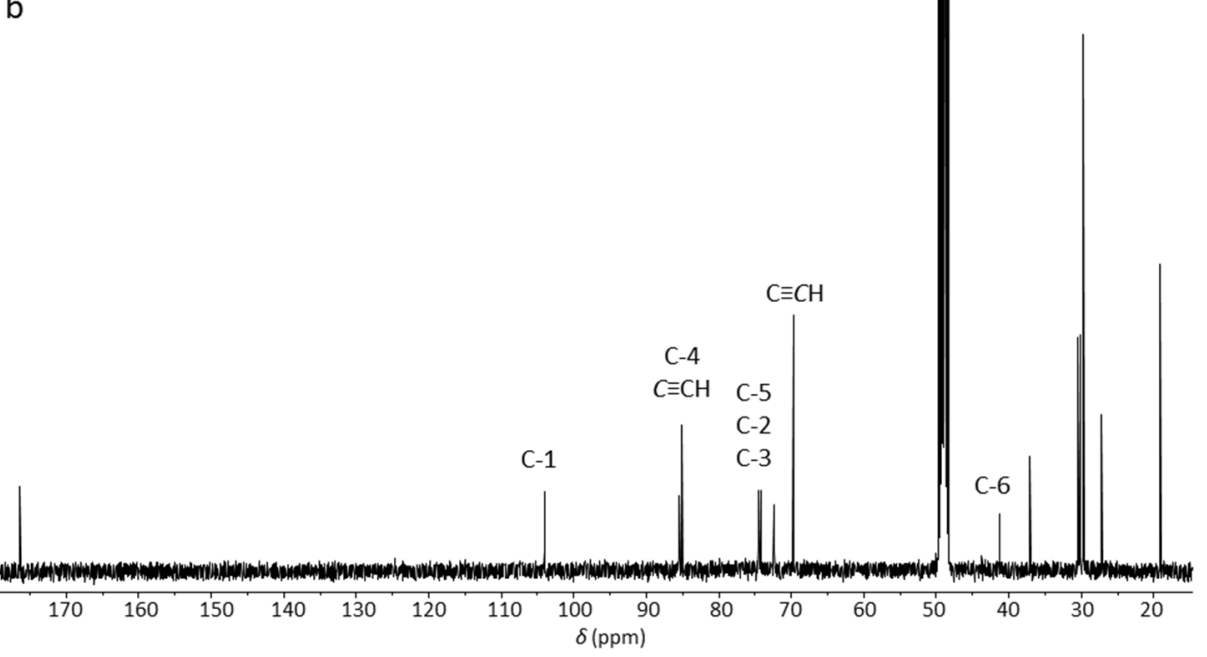

Figure 7.5. a) ${ }^{1} \mathrm{H}$ and b) ${ }^{13} \mathrm{C}$ NMR spectra of 1 (measured at 400 and $100.3 \mathrm{MHz}$, respectively, in methanol- $d_{4}$ ). Numbered signals correspond to the cyclodextrin ring.

$\mathrm{CD}$ monolayer formation on silicon. A $4 \mathrm{mM}$ solution of heptaalkyne-functionalized $\beta-C D$ 1 in THF/DMF/mesitylene $(0.3 / 0.8 / 3.0 \mathrm{~mL})$ was degassed by four freeze-pump-thaw cycles. Silicon $\mathrm{p}$ and $\mathrm{p}^{++}$substrates were diced into pieces of $2 \times 2 \mathrm{~cm}^{2}$ and sonicated in acetone for $10 \mathrm{~min}$ to remove particles generated during dicing. Native oxide was removed from $\mathrm{p}$ and $\mathrm{p}^{++}$silicon substrates by immersion in 1\% aqueous HF for 2 min to result in an $\mathrm{H}$-terminated surface. The back side was sputtered with a $1 \mu \mathrm{m}$ aluminum/silicon layer (99/1\% Al/Si, Oxford PL400, $7 \mathrm{~kW}$ ) as Ohmic contact for electrochemistry. Afterwards, the substrates were immediately immersed in the 
degassed solution of 1 inside a nitrogen glovebox. The reaction flask was equipped with a capillary as a nitrogen inlet and a reflux condenser. The hydrosilylation reaction was performed overnight under continuous nitrogen flow at $60^{\circ} \mathrm{C}$. The wafers were cleaned by 2 min ultrasonication in dichloromethane, rinsing with ethanol, rinsing with Milli- $Q$ water, and subsequently dried in a stream of nitrogen.

Guest immobilization. A $10 \mu \mathrm{M}$ guest solution was made by first dissolving guest 2 in methanol, followed by dilution with water to obtain a solution with $1 \% \mathrm{v} / \mathrm{v}$ methanol. Host-functionalized surfaces were incubated with guest molecules by 15 min immersion in this solution. The surfaces were rinsed with Milli- $Q$ water, $2 \mathrm{~mL}$ of $1 \mathrm{mM}$ aqueous $\beta-C D$ solution, and Milli-Q water, and dried in a stream of nitrogen. Competition experiments were performed by $2 \times 15 \mathrm{~min}$ immersion in a $10 \mathrm{mM}$ aqueous $\beta-C D$ solution.

\subsubsection{Equipment}

Characterization after synthesis. ${ }^{1} \mathrm{H}$ and ${ }^{13} \mathrm{C}$ NMR were recorded at 400 and $100.6 \mathrm{MHz}$, respectively. 2D COSY and HMQC experiments were used to assist the NMR peak assignments. Fourier transform infrared (FT-IR) spectroscopy was performed on a Nicolet 6700 FT-IR instrument $\left(4 \mathrm{~cm}^{-1}\right.$ resolution, 128 scans). Thin-layer chromatography was carried out on aluminum sheets, with visualization by UV light and by charring with $10 \% \mathrm{H}_{2} \mathrm{SO}_{4}$. Column chromatography was carried out on silica gel (230-400 mesh). ESI-MS spectra were obtained for samples dissolved in dichloromethane-methanol at low $\mu \mathrm{M}$ concentrations.

Contact angle measurements. Static contact angles were measured with Milli-Q water on a Krüss G10 Contact Angle Measuring Instrument equipped with a CCD camera. Contact angle values were determined automatically by a drop shape analysis software. Contact angles were measured directly after the hydrosilylation reaction or guest immobilization and shown as an average of four drops.

Attenuated total reflection infrared spectroscopy. ATR-IR spectra were measured on double side polished silicon wafer pieces treated equally to the electrochemistry samples but lacking the aluminum/silicon contact. Spectra were collected with a Bruker spectrometer (Vertex 70v) equipped with an attenuated total reflection accessory $\left(L=15 \mathrm{~mm}\right.$ ) and MCT detector. The spectra were recorded with a resolution of $4 \mathrm{~cm}^{-1}$ and 1024 scans and treated with a spline baseline correction. Each spectrum was referenced to a hydrogen-terminated silicon sample.

X-ray photoelectron spectroscopy. XPS measurements were performed on a Quantera SXM setup from Physical Electronics equipped with an Al Ka X-ray source (1486.6 eV). A 
detector angle of $45^{\circ}$ was used, and collected spectra were calibrated on the C1s peak at $284.8 \mathrm{eV}$.

Electrochemistry. Electrochemical measurements were performed on a $\mathrm{CH}$ Instruments bipotentiostat 760D. Measurements were performed in a custom-built glass electrochemical cell with a platinum disk as counter electrode, a red rod reference electrode $(\mathrm{Ag} / \mathrm{AgCl}$, saturated $\mathrm{KCl}$ solution, Radiometer Analytical), and the functionalized silicon $\mathrm{p}$ or $\mathrm{p}^{++}$substrates as working electrode $\left(0.44 \mathrm{~cm}^{2}\right)$. Cyclic voltammograms were recorded in $1 \mathrm{M}$ sodium perchlorate monohydrate $\left(\mathrm{NaClO}_{4}\right)$ in water at scan rates of 0.1 , $0.2,0.4,0.6,0.8,1.0 \mathrm{~V} / \mathrm{s}$. Three cycles were recorded, of which the last is shown in the graphs. An electrochemical titration of guest 2 were performed by measuring cyclic voltammetry on host-functionalized surfaces with on top aqueous solutions with $1 \mathrm{mM}$ $\beta-C D, 1 \mathrm{M} \mathrm{NaClO}_{4}$ and different concentrations of guest $2(0.1,0.5,1,2,4,6,8 \mu \mathrm{M})$. To prepare the guest solutions, a stock solution with $15 \mu \mathrm{M}$ guest was sonicated for $30 \mathrm{~min}$ to dissolve the guest in water with $1 \mathrm{mM} \beta-\mathrm{CD}$ and $1 \mathrm{M} \mathrm{NaClO}_{4}$, which was then mixed in different ratios with the guest-free solution. The surface coverage was determined for each guest concentration by measuring at scan rates of $0.1,0.2,0.4,0.6,0.8,1.0 \mathrm{~V} / \mathrm{s}$. For all measurements, peak currents were determined by Gaussian fitting (using linear baseline correction) in the CHI760D software. The voltammetric data were recorded with an accuracy of $10 \mu \mathrm{A}$ and $5 \mathrm{mV}$.

\subsection{References}

1. Y.L. Wang, T.Y. Wang, P.M. Da, M. Xu, H. Wu and G.F. Zheng, Adv. Mater., 2013, 25, 51775195.

2. B. Fabre, Y. Li, L. Scheres, S.P. Pujari and H. Zuilhof, Angew. Chem., Int. Ed., 2013, 52, 1202412027.

3. M.N. Masood, S. Chen, E.T. Carlen and A. van den Berg, ACS Appl. Mater. Interfaces, 2010, 2, 3422-3428.

4. L. Basabe-Desmonts, J. Beld, R.S. Zimmerman, J. Hernando, P. Mela, M.F. Garcia-Parajo, N.F. van Hulst, A. van den Berg, D.N. Reinhoudt and M. Crego-Calama, J. Am. Chem. Soc., 2004, 126, 7293-7299.

5. M.D. Yilmaz, S.H. Hsu, D.N. Reinhoudt, A.H. Velders and J. Huskens, Angew. Chem., Int. Ed., 2010, 49, 5938-5941.

6. C.A. Nijhuis, B.J. Ravoo, J. Huskens and D.N. Reinhoudt, Coord. Chem. Rev., 2007, 251, 17611780.

7. C.Z. Zhu and L. Fang, Curr. Org. Chem., 2014, 18, 1957-1964.

8. S. Onclin, A. Mulder, J. Huskens, B.J. Ravoo and D.N. Reinhoudt, Langmuir, 2004, 20, 54605466.

9. A. Méndez-Ardoy, T. Steentjes, T. Kudemac and J. Huskens, Langmuir, 2014, 30, 3467-3476.

10. M.W.J. Beulen, J. Bügler, B. Lammerink, F.A.J. Geurts, E.M.E.F. Biemond, K.G.C. van Leerdam, F.C.J.M. van Veggel, J.F.J. Engbersen and D.N. Reinhoudt, Langmuir, 1998, 14, 6424-6429. 
11. A. Méndez-Ardoy, T. Steentjes, B.A. Boukamp, P. Jonkheijm, T. Kudernac and J. Huskens, Langmuir, 2016, in press, DOI: 10.1021/acs.langmuir.6b03860.

12. M.W.J. Beulen, J. Bugler, M.R. de Jong, B. Lammerink, J. Huskens, H. Schonherr, G.J. Vancso, B.A. Boukamp, H. Wieder, A. Offenhauser, W. Knoll, F.C.J.M. van Veggel and D.N. Reinhoudt, Chem. Eur. J., 2000, 6, 1176-1183.

13. V. Kolivoška, R. Sokolová, J. Kocábová, C. Loukou, J.-M. Mallet and M. Hromadová, Monatsh. Chem., 2016, 147, 45-51.

14. L.T. Yang, A. Gomez-Casado, J.F. Young, H.D. Nguyen, J. Cabanas-Danes, J. Huskens, L. Brunsveld and P. Jonkheijm, J. Am. Chem. Soc., 2012, 134, 19199-19206.

15. Z.X. Zhang, J.A. Wang, X.L. Wang, Y. Wang and X.R. Yang, Talanta, 2010, 82, 483-487.

16. C. Quintana, C.S.H. Domínguez, E. Blanco, P. Hernández and L. Hernández, Microchim. Acta, 2010, 171, 41-47.

17. X.Y. Ling, D.N. Reinhoudt and J. Huskens, Chem. Mater., 2008, 20, 3574-3578.

18. C.A. Nijhuis, J. Huskens and D.N. Reinhoudt, J. Am. Chem. Soc., 2004, 126, 12266-12267.

19. M.J.W. Ludden, J.K. Sinha, G. Wittstock, D.N. Reinhoudt and J. Huskens, Org. Biomol. Chem., 2008, 6, 1553-1557.

20. C.A. Nijhuis, J.K. Sinha, G. Wittstock, J. Huskens, B.J. Ravoo and D.N. Reinhoudt, Langmuir, 2006, 22, 9770-9775.

21. L. Chen, L. Tian, L. Liu, X. Tian, W. Song, H. Xu and X. Wang, Sens. Actuators, B, 2005, 110, 271278.

22. C. Lagrost, G. Alcaraz, J.F. Bergamini, B. Fabre and I. Serbanescu, Chem. Commun., 2007, 10501052.

23. B. Fabre, Chem. Rev., 2016, 116, 4808-4849.

24. A.B. Sieval, R. Linke, H. Zuilhof and E.J.R. Sudholter, Adv. Mater., 2000, 12, 1457-1460.

25. J.M. Buriak, Chem. Commun., 1999, 1051-1060.

26. V. Vrkoslav, I. Jelinek, T. Trojan, J. Jindrich and J. Dian, Phys. E, 2007, 38, 200-204.

27. T. Trojan, J. Jindrich, V. Vrkoslav, I. Jelinek and J. Dian, J. Inclusion Phenom. Macrocyclic Chem., 2007, 57, 343-348.

28. L. Scheres, M. Giesbers and H. Zuilhof, Langmuir, 2010, 26, 10924-10929.

29. A. Ng, S. Ciampi, M. James, J.B. Harper and J.J. Gooding, Langmuir, 2009, 25, 13934-13941.

30. M.R. de Jong, J. Huskens and D.N. Reinhoudt, Chem. Eur. J., 2001, 7, 4164-4170.

31. S. Ciampi, M. James, P. Michaels and J.J. Gooding, Langmuir, 2011, 27, 6940-6949.

32. N.S. Bhairamadgi, S. Gangarapu, M.A. Caipa Campos, J.M.J. Paulusse, C.J.M. van Rijn and H. Zuilhof, Langmuir, 2013, 29, 4535-4542.

33. N. Husken, M. Gebala, F. La Mantia, W. Schuhmann and N. Metzler-Nolte, Chem. Eur. J., 2011, 17, 9678-9690.

34. E. Laviron, J. Electroanal. Chem. Interfacial Electrochem., 1979, 101, 19-28.

35. A.J. Bard and L.R. Faulkner, Electrochemical methods. Fundamentals and Applications, 2nd ed., Wiley, 2001, p. 591.

36. A. Mulder, T. Auletta, A. Sartori, S. Del Ciotto, A. Casnati, R. Ungaro, J. Huskens and D.N. Reinhoudt, J. Am. Chem. Soc., 2004, 126, 6627-6636.

37. J. Huskens, A. Mulder, T. Auletta, C.A. Nijhuis, M.J.W. Ludden and D.N. Reinhoudt, J. Am. Chem. Soc., 2004, 126, 6784-6797.

38. R. Zanoni, F. Cattaruzza, C. Coluzza, E.A. Dalchiele, F. Decker, G. Di Santo, A. Flamini, L. Funari and A.G. Marrani, Surf. Sci., 2005, 575, 260-272. 
39. F. Decker, F. Cattaruzza, C. Coluzza, A. Flamini, A.G. Marrani, R. Zanoni and E.A. Dalchiele, J. Phys. Chem. B, 2006, 110, 7374-7379.

40. V.R. Goncales, Y.F. Wu, B. Gupta, S.G. Parker, Y. Yang, S. Ciampi, R. Tilley and J.J. Gooding, J. Phys. Chem. C, 2016, 120, 15941-15948.

41. G.G. Condorelli, A. Motta, M. Favazza, E. Gurrieri, P. Betti and E. Dalcanale, Chem. Commun., 2010, 46, 288-290.

42. P.R. Ashton, R. Königer, J.F. Stoddart, D. Alker and V.D. Harding, J. Org. Chem., 1996, 61, 903908. 


\section{Chapter 8}

\section{Covalent and noncovalent immobilization of hydrogen evolution catalysts on gold and silicon electrodes}

Solar-to-fuel devices require the immobilization of hydrogen and oxygen evolution catalysts. We have investigated two routes to achieve immobilization of a molecular catalyst with a [FeFe]hydrogenase mimic as the active headgroup for hydrogen evolution. First, covalent attachment was tested by hydrosilylation of an alkyne-functionalized hydrogenase mimic onto H-terminated silicon surfaces. Second, supramolecular hostguest interactions were used to achieve immobilization of a guest-functionalized catalyst onto $\beta-C D$ monolayers on gold and silicon substrates. The supramolecular catalyst was synthesized with three adamantyl moieties for strong anchoring to the substrate. For both routes, catalyst immobilization was confirmed by infrared spectroscopy and X-ray photoelectron spectroscopy, which showed the characteristic $\mathrm{C} \equiv \mathrm{O}$ stretch vibrations and $\mathrm{N}, \mathrm{S}$, and Fe atoms of the hydrogenase mimic, respectively. Ellipsometry showed that the covalently bound catalyst formed multilayers on the surface. Surface plasmon resonance spectroscopy was used to assess the binding affinity of the supramolecular catalyst, which indicated that the host-guest interactions are effectively divalent. Preliminary electrochemistry experiments indicated some reduction activity of both catalysts, but more research is needed to verify whether the hydrogenase mimics are still catalytically active after immobilization.
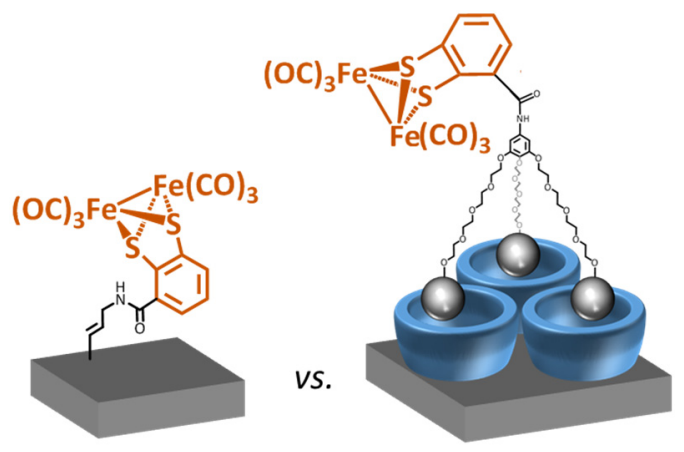


\subsection{Introduction}

Solar-to-fuel devices, i.e., devices that convert water or $\mathrm{CO}_{2}$ into an energy carrier using sunlight, have gained increasing attention due to the world's need for more sustainable energy sources. ${ }^{1-3}$ These photoelectrochemical devices are often designed as a tandem setup, in which two semiconductors are assembled in series, and in which the two electrodes have a membrane in between, and catalysts are immobilized on either side to assist water splitting. ${ }^{4,5}$ One of the steps required in device fabrication is the immobilization of a catalyst onto the surface on an electrode.

The most feasible route to bind a catalyst to a surface depends on the nature of the catalyst, i.e., whether it is in a particle form or as a molecular catalyst. Molecular catalysts are attractive because of their high reactivity and selective catalysis. ${ }^{6}$ These, mostly transition metal-based, complexes have been immobilized for hydrogen evolution, ${ }^{5,7-10}$ or $\mathrm{CO}_{2}$ reduction, ${ }^{6}$ for example. Here, we focus on a hydrogenase mimic as a molecular hydrogen evolution catalyst. Hydrogenases are naturally occurring enzymes that are able to reversibly reduce protons to molecular hydrogen with a rate and overpotential similar to platinum, which is the best inorganic catalyst known to date. ${ }^{11}$ Iron-iron hydrogenases ([FeFe]hydrogenase) are the most efficient of this class of enzymes, which makes it fruitful to mimic these enzymes synthetically. ${ }^{11-13}$ The ideal mimic has not been found yet, as in most cases the synthetic catalyst operates at a high overpotential and/or shows a low production rate. ${ }^{14}$ Moreover, many mimics are poorly soluble in water, whereas water is highly desirable as the proton source. ${ }^{15}$ One of the main mimics used involves a [FeFe] core to which six carbonyl ligands are bound and a benzenedithiolate bridge to form a $\mathrm{Fe}_{2} \mathrm{~S}_{2}$ cluster.

Catalyst coupling can be obtained covalently, i.e., by covalent monolayer formation, or noncovalently, i.e., by supramolecular host-guest interactions. Covalent bond formation leads to irreversible fixation of molecules, which avoids undesired catalyst desorption. To allow electronic coupling between the catalyst and the substrate, an insulating layer of silicon oxide should be avoided. For this purpose, hydrosilylation is a proper method, which is a one-step reaction in which molecules with unsaturated carbon-carbon end groups are coupled to hydrogen-terminated silicon. ${ }^{16,17}$ In this way, electrochemical measurements on, for example, ferrocene-containing monolayers have been reported. ${ }^{18-20}$ On the other hand, noncovalent interactions offer modularity and tunability. ${ }^{21,22}$ Specifically, $\beta$-cyclodextrin $(\beta-C D)$ is a suitable host molecule for the formation of self-assembled monolayers with molecular recognition properties. These cyclic cavities can be used to form stable monolayers on several substrates, including gold, ${ }^{23,24}$ glass, ${ }^{25}$ and silicon, ${ }^{26}$ due to their heptapodant nature. In Chapter 7 we have shown that electronic coupling between a redox-active guest and $\beta-C D$ monolayers on silicon is possible, which makes these platforms also applicable for catalyst 
immobilization. Strong host-guest interactions can be obtained using guest molecules with adamantyl moieties. ${ }^{25,27}$ Based on the valency of the guest, the strength of the hostguest interactions can be tuned. ${ }^{28,29}$ When employing competition with free host molecules in solution, the catalyst could be removed and reloaded at will.

Here, we study the immobilization of a hydrogenase mimic as a molecular hydrogen evolution catalyst by two routes, i.e., both covalently by hydrosilylation and noncovalently on host monolayers of $\beta-C D$. As a substrate, silicon was used because it is a suitable semiconductor for the hydrogen production side of a solar-to-fuel device. Gold substrates were tested as well, because of its possible use in solar-to-fuel devices, for example as gold nanoparticles on a semiconductor device. ${ }^{30}$ The [FeFe]hydrogenase mimic for covalent binding was functionalized with an alkyne moiety to allow hydrosilylation onto H-terminated silicon. The supramolecular catalyst was designed with a tris-adamantyl moiety, since such trivalent interactions would enable strong immobilization on a $\beta-C D$ surface. For both routes, the immobilization was characterized using contact angle measurements, infrared spectroscopy, and X-ray photoelectron spectroscopy (XPS). Electrochemistry was used to characterize the redox properties and catalytic activity of the catalyst.

\subsection{Results and discussion}

A schematic procedure for monolayer formation, either by covalent or noncovalent binding of a hydrogenase mimic, is shown in Scheme 8.1. The alkyne-functionalized hydrogenase mimic 1 was coupled directly onto $\mathrm{H}$-terminated silicon by hydrosilylation (Si-1, Scheme 8.1a). ${ }^{16,17} \beta$-CD monolayers on gold were formed, as reported before, using heptaiodo-functionalized $\beta-C D 2$ (Au-2, Scheme 8.1b). ${ }^{23}$ Silicon substrates were functionalized as described in Chapter 7, i.e., by hydrosilylation of heptaalkynefunctionalized $\beta$-CD 3 onto H-terminated silicon (Si-3, Scheme 8.1c). Tris-adamantyl hydrogenase mimic 5 (Scheme 8.1d) consisted of three tetra(ethylene glycol) spacers with adamantyl moieties for stable anchoring and water solubility and a redox active hydrogenase mimic moiety linked through a benzene ring. Bis-adamantyl ferrocene guest 4 , as also used in Chapter 7, was used as a model compound to probe the stability of the host-guest monolayers in the potential range where guest 5 was redox active. 


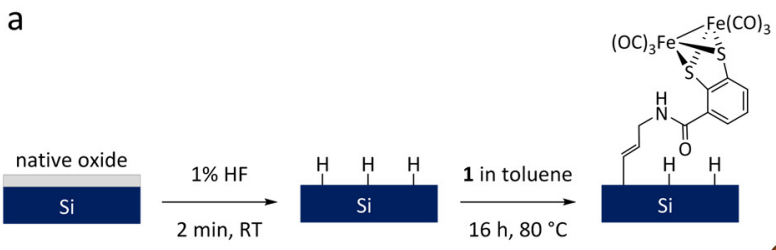

b

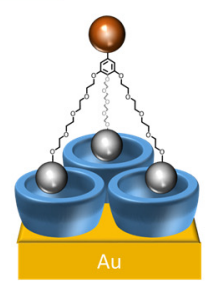

C
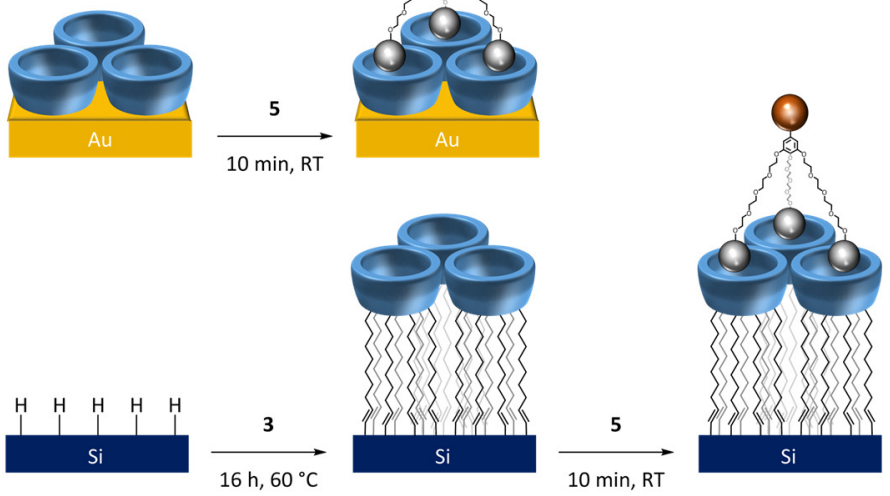

d
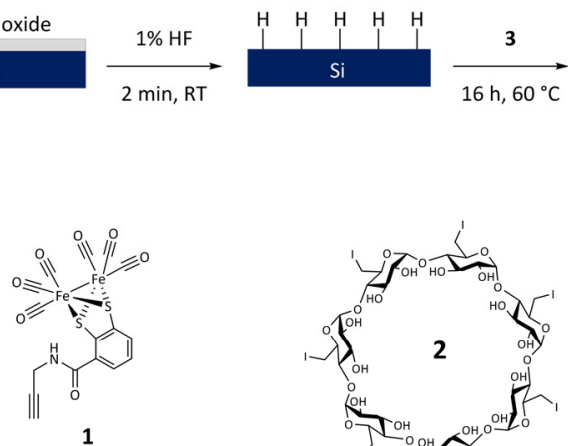

$10 \min , \mathrm{RT}$
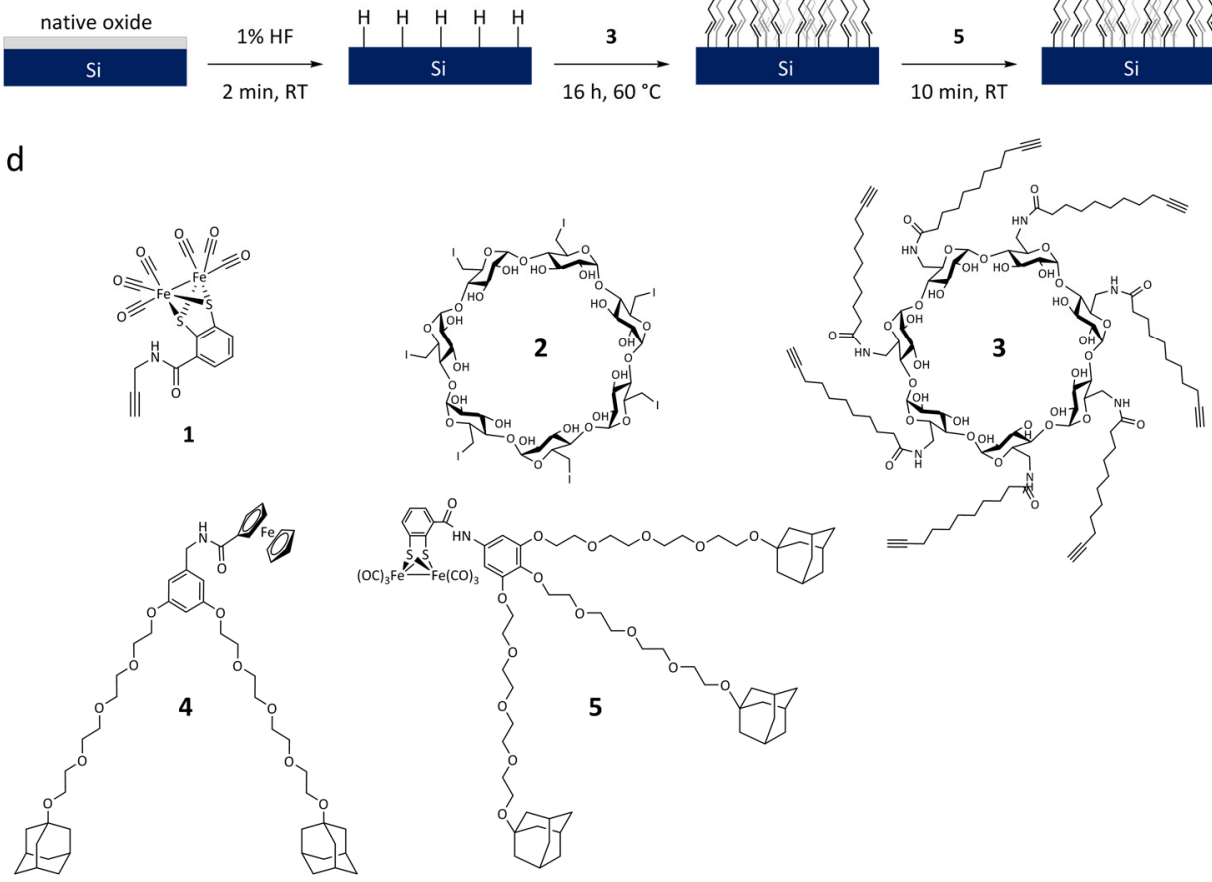

Scheme 8.1. a) Schematic procedure of silicon surface functionalization by covalent coupling of 1 onto $\mathrm{H}$-terminated $\mathrm{Si}$. b) Schematic procedure of gold surface functionalization by monolayer formation of 2, followed by host-guest binding of 5. c) Schematic procedure of silicon surface functionalization by covalent coupling of 3 onto $\mathrm{H}$-terminated $\mathrm{Si}$, followed by host-guest binding of 5. d) Chemical structures of alkyne-functionalized hydrogenase mimic 1 , heptaiodo-functionalized $\beta-C D 2$, heptaalkyne-functionalized $\beta-C D$ 3, bis-adamantyl ferrocene guest 4 , and tris-adamantyl hydrogenase mimic 5 . 


\subsubsection{Covalently bound hydrogenase mimic}

The alkyne-functionalized hydrogenase mimic 1 (Scheme 8.1d) was synthesized by

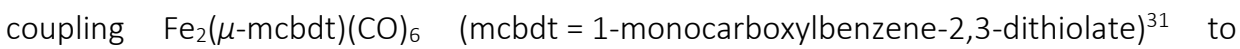
propargylamine. ${ }^{1} \mathrm{H}$ nuclear magnetic resonance (NMR) spectroscopy confirmed the coupling, although some impurities were present in the $0.5-2 \mathrm{ppm}$ region as well (see Figure 8.9 in the Experimental Section). Fourier transform infrared spectroscopy (FT-IR) showed the presence of the characteristic $C \equiv 0$ stretch vibrations at 2080,2043 , and $2001 \mathrm{~cm}^{-1}$ (Figure 8.1), similar to spectra reported in the literature. ${ }^{12,32}$ This compound was coupled onto $\mathrm{H}$-terminated $\mathrm{Si}$ by a hydrosilylation reaction (Scheme 8.1a). The native oxide layer was removed from lowly doped ( $\mathrm{p}$-type) or highly doped $\left(\mathrm{p}^{++}\right)$Si substrates by short immersion in $1 \%$ aqueous hydrofluoric acid (HF). After sputtering an aluminum/silicon alloy as Ohmic contact at the back side, the substrates were immersed in a $2 \mathrm{mM}$ solution of 1 in toluene overnight at $80^{\circ} \mathrm{C}$. This resulted in static water contact angles that varied from batch to batch. Values in the range of $78-99^{\circ}$ and $67-97^{\circ}$ were found for $\mathrm{Si}(\mathrm{p})-1$ and $\mathrm{Si}\left(\mathrm{p}^{++}\right)-1$ substrates (4 batches), respectively. The standard deviations within samples were about $3^{\circ}$, which is relatively high and indicates an inhomogeneous surface functionalization. Nonetheless, attenuated total reflection infrared spectroscopy (ATR-IR, Figure 8.1) confirmed Si-1 formation by the presence of the $\mathrm{C} \equiv \mathrm{O}$ stretches in the $2080-2000 \mathrm{~cm}^{-1}$ region, which indicates that the catalyst is still intact after immobilization. The formation of Si-1 was also confirmed by the appearance of $\mathrm{C}-\mathrm{H}$ stretches at 2962 and $2930 \mathrm{~cm}^{-1}$. The broad peak at $3294 \mathrm{~cm}^{-1}$, which is hardly visible in the spectrum of pure 1 , probably originates from the $\mathrm{N}-\mathrm{H}$ stretch of the secondary amide group. The $\mathrm{C} \equiv \mathrm{CH}$ stretch vibration would also appear in this region for any unreacted or physisorbed molecules of 1 but should result in a sharper peak. Moreover, any physisorbed molecules are expected to be removed by ultrasonication in toluene, dichloromethane, and acetonitrile.

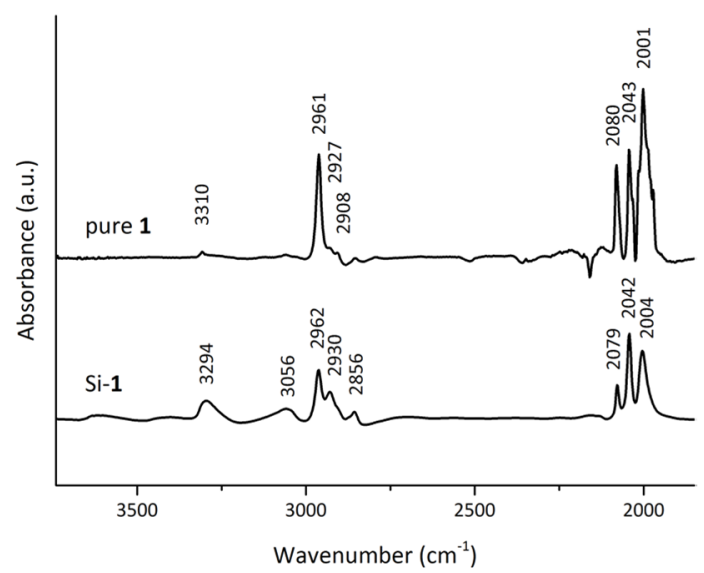

Figure 8.1. ATR-IR spectra of a silicon substrate functionalized with $1(\mathrm{Si}(\mathrm{p})-1)$ referenced to an $\mathrm{H}$-terminated silicon sample. The FT-IR spectrum of pure 1 is added for comparison. 
XPS of Si-1 surfaces showed the presence of N, S, and Fe atoms (Table 8.1). This indicates, together with the $\mathrm{C} \equiv \mathrm{O}$ stretches observed by $\mathrm{ATR}-\mathrm{IR}$, that the molecules were immobilized on the silicon substrates. For retaining the catalytic activity, intactness of the $\mathrm{Fe}_{2} \mathrm{~S}_{2}$ center is of importance. The $\mathrm{N}: \mathrm{S}:$ Fe ratios equaled to 1:1.5:2.8 and 1:2.0:3.0 for $\mathrm{Si}(\mathrm{p})-1$ and $\mathrm{Si}\left(\mathrm{p}^{++}\right)-1$, respectively, which slightly deviated from the expected ratio of 1:2:2 based on the molecular structure of 1 . This deviation could be explained by overlap of the S2p and Si2s peaks, which required indirect quantification of the S contents and resulted in an error of the atomic percentage of $\mathrm{S}$. The deviating ratio was also observed in the XPS spectrum of physisorbed molecules of 1 on Si (Table 8.1, ratio 1:1.3:1.0), which indicates an XPS sensitivity issue rather than damage to the immobilized molecules. Thus, we conclude that the Si-1 surfaces have been formed successfully and that the molecules seemed to be intact after immobilization.

Table 8.1. Relative atomic percentages from XPS measurements on Si-1 surfaces or with 1 physisorbed on $\mathrm{Si}(\mathrm{p})$.

$\begin{array}{lcccccc}\text { Sample } & \text { Si (\%) } & \mathrm{C}(\%) & \mathrm{O}(\%) & \mathrm{N}(\%) & \mathrm{S}(\%) & \mathrm{Fe}(\%) \\ \text { Si }(\mathrm{p})-1 & 9.0 \pm 0.5 & 49.9 \pm 0.2 & 25.2 \pm 0.6 & 2.6 \pm 0.5 & 3.9 \pm 0.3 & 7.2 \pm 0.2 \\ \text { Si }\left(\mathrm{p}^{++}\right)-1 & 5.8 \pm 0.6 & 53.0 \pm 0.5 & 25.2 \pm 0.5 & 2.7 \pm 0.3 & 5.4 \pm 0.1 & 8.0 \pm 0.3 \\ \text { 1 on Si(p) } & 8.1 \pm 0.4 & 68.1 \pm 0.6 & 17.2 \pm 0.4 & 2.0 \pm 0.2 & 2.6 \pm 0.5 & 2.0 \pm 0.1\end{array}$

The N, S, and Fe atoms were present in relatively high amounts, whereas the amount of silicon detected was rather low $(<10 \%)$ compared to XPS spectra of other monolayers on $\mathrm{Si}$. This could denote multilayer formation. This hypothesis was confirmed by ellipsometry measurements, since Si-1 substrates showed layer thicknesses of 7.1-9.6 nm, whereas $\sim 1 \mathrm{~nm}$ was expected. Multilayer formation could have occurred by repetitive alkyne addition, ${ }^{33}$ although this has not been observed for hydrosilylation of other 1-alkynes onto $\mathrm{Si}-\mathrm{H}$ (including the heptapodant $\beta-\mathrm{CD}$ derivative described below), and steric hindrance is expected to impede the approach of additional molecules of 1 towards the Si-C bond.

\subsubsection{Supramolecularly bound hydrogenase mimic}

$\beta-C D$ monolayers were formed on cleaned gold substrates by overnight immersion in a solution of heptaiodo-functionalized $\beta-C D$ (Scheme $8.1 \mathrm{~b}) .{ }^{23}$ To create Si-3 monolayers (Scheme 8.1c), $\mathrm{p}$ and $\mathrm{p}^{++}$silicon substrates with an aluminum/silicon alloy as Ohmic contact at the back side were used. On these substrates, $\beta-C D$ monolayers were obtained by immersing Si-H substrates in a $4 \mathrm{mM}$ solution of 3 in THF/DMF/mesitylene overnight at $60^{\circ} \mathrm{C}$ (see Chapter 7). Static water contact angle measurements showed values of $31.2^{\circ} \pm 1.7,77.2^{\circ} \pm 0.9$, and $72.2^{\circ} \pm 3.2$ for $\mathrm{Au}-2$, Si $(\mathrm{p})-3$, and $\mathrm{Si}\left(\mathrm{p}^{++}\right)-3$, respectively. The value for the gold substrate was lower than expected, since an (advancing) contact angle of $45^{\circ}$ was reported before for the same monolayers. ${ }^{23}$ The values on silicon were 
comparable to those reported in Chapter 7. Infrared reflection-absorption spectroscopy (IRRAS) of Au-2 samples (Figure 8.2) did not reveal any peaks in the range of 3700$1900 \mathrm{~cm}^{-1}$, which is similar to the data reported before. ${ }^{23}$ ATR-IR of silicon dummy substrates displayed a similar spectrum as shown in Figure 7.1. The formation of Si-3 was confirmed by the appearance of $\mathrm{O}-\mathrm{H} / \mathrm{N}-\mathrm{H}$ stretch vibrations in the $3600-3200 \mathrm{~cm}^{-1}$ range, $\mathrm{C}-\mathrm{H}$ stretches at 2962, 2918, $2853 \mathrm{~cm}^{-1}$, and the disappearance of $\mathrm{Si}-\mathrm{H}_{\mathrm{x}}$ stretches around $2112 \mathrm{~cm}^{-1}$ (Figure 8.2). The absence of any $\mathrm{C} \equiv \mathrm{CH}$ stretch vibration at $3300 \mathrm{~cm}^{-1}$ indicated that the alkyne groups had fully reacted onto the surface.

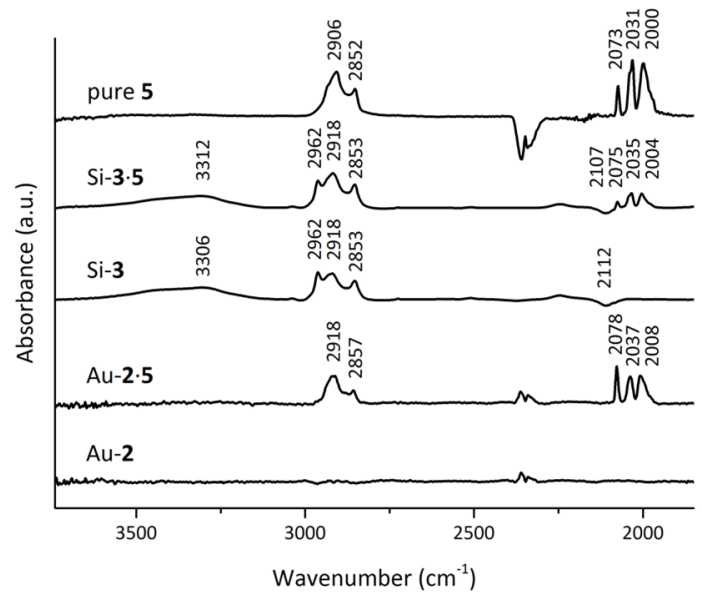

Figure 8.2. IR spectra of gold (IRRAS) and silicon (ATR-IR) substrates functionalized with $\beta$-CD (Au-2 and $\mathrm{Si}(\mathrm{p})-3)$ and with host-guest complexes of guest $5(\mathrm{Au}-2 \cdot 5$, $\mathrm{Si}(\mathrm{p})-3 \cdot 5)$. The spectra on silicon samples are referenced to an $\mathrm{H}$-terminated silicon sample. The FT-IR spectrum of pure 5 is added for comparison.

Hydrogenase mimics such as guest 5 show electron transfer around $-1.0 \mathrm{~V} v \mathrm{vs} . \mathrm{Ag} / \mathrm{AgCl}$, where the exact potential depends on the specific [FeFe] complex and (the $\mathrm{pH}$ of) the solvent used. ${ }^{15,34-36}$ The stability of the host-guest monolayers was tested under these strongly reducing conditions, which could possibly be destructive for the $\beta-C D$ monolayer and/or the $\beta-C D /$ adamantane interactions. Stability tests were performed using bisadamantyl ferrocene guest 4 as a model compound. The guest surface coverage was determined from the cyclic voltammograms at different scan rates, as described in Chapter 7. These guest coverages equaled to $1.9 \times 10^{-11} \mathrm{~mol} / \mathrm{cm}^{2}$ and $1.4 \times 10^{-11} \mathrm{~mol} / \mathrm{cm}^{2}$ for Au-2.4 and Si-3.4 monolayers, respectively. Assuming divalent guest binding as reported before, ${ }^{37}$ the host coverages equaled to $3.8 \times 10^{-11} \mathrm{~mol} / \mathrm{cm}^{2}$ and $2.8 \times 10^{-11}$ $\mathrm{mol} / \mathrm{cm}^{2}$, respectively. These are somewhat lower than reported before for equal or comparable $\beta-C D$ monolayers, i.e., $5.4 \times 10^{-11} \mathrm{~mol} / \mathrm{cm}^{2}$ for Au-2.4 monolayers and $5.9 \times 10^{-11} \mathrm{~mol} / \mathrm{cm}^{2}$ for alkyl-functionalized $\beta-C D$ molecules on gold, respectively. ${ }^{37}$ 
The stability of Au-2.4 and Si-3.4 monolayers was electrochemically characterized by measuring the guest coverage of 4 after taking several cyclic voltammetry (CV) cycles in the potential range down to $-1.1 \mathrm{~V}$ vs. $\mathrm{Ag} / \mathrm{AgCl}$. For $\mathrm{Au}-2 \cdot 4$ substrates, four cycles performed at $1 \mathrm{~V} / \mathrm{s}$ in the potential range 0.0 to $-1.1 \mathrm{~V} \mathrm{vs}$. $\mathrm{Ag} / \mathrm{AgCl}$ led to only a slight decrease of the guest coverage from $1.9 \times 10^{-11} \mathrm{~mol} / \mathrm{cm}^{2}$ to $1.8 \times 10^{-11} \mathrm{~mol} / \mathrm{cm}^{2}$. CV over a more positive potential range including the redox potential of the ferrocene moiety $(0.8$ to $-1.1 \mathrm{~V} v \mathrm{~s}$. $\mathrm{Ag} / \mathrm{AgCl}$ ), did decrease the surface coverage further to $7.7 \times 10^{-12} \mathrm{~mol} / \mathrm{cm}^{2}$ after four additional cycles (about $40 \%$ of the original coverage remaining) and to nondetectable amounts after another ten cycles. Incubation with a fresh guest (4) solution replenished the surface coverage to $8.4 \times 10^{-12} \mathrm{~mol} / \mathrm{cm}^{2}$ (about $44 \%$ of the original amount), which indicates that there were still host molecules on the surface available for host-guest interactions. The stability was better on $\mathrm{Si}\left(\mathrm{p}^{++}\right)-3 \cdot 4$ substrates, where the guest coverage decreased from $1.4 \times 10^{-11} \mathrm{~mol} / \mathrm{cm}^{2}$ to $8.9 \times 10^{-12} \mathrm{~mol} / \mathrm{cm}^{2}$ (64\%) after ten cycles from 0 to $-1.1 \mathrm{~V}$ vs. $\mathrm{Ag} / \mathrm{AgCl}$ and then to $6.9 \times 10^{-12} \mathrm{~mol} / \mathrm{cm}^{2}(50 \%)$ after ten additional cycles from 0.8 to $-1.1 \mathrm{~V}$ vs. $\mathrm{Ag} / \mathrm{AgCl}$. On the silicon substrates, a lower loss in surface coverage was thus observed compared to the gold substrates. These experiments showed that the host-guest monolayers on both $\mathrm{Au}$ and Si were relatively stable in the potential window needed $(0.0$ to $-1.1 \mathrm{Vvs}$. $\mathrm{Ag} / \mathrm{AgCl})$, and the use of $\beta-\mathrm{CD}$ monolayers as a platform to immobilize the hydrogenase mimic 5 should thus be feasible.

As a supramolecular guest molecule, tris-adamantyl hydrogenase mimic 5 (Scheme $8.1 \mathrm{~d}$ ) was synthesized by coupling $\mathrm{Fe}_{2}(\mu$-mcbdt $)(\mathrm{CO})_{6}{ }^{31}$ to a trivalent adamantane guest with an amino headgroup. ${ }^{29}$ Formation of the desired product was confirmed by mass spectrometry and ${ }^{1} \mathrm{H}$ NMR spectroscopy (see Figure 8.10 in the Experimental Section). Upon coupling of the starting compounds, the ${ }^{1} \mathrm{H}$ NMR spectrum showed a shift of the aromatic protons from the aniline ring from $6.32 \mathrm{ppm}$ to $7.05 \mathrm{ppm}$, which indicates successful coupling. Next, the glycol chains were still bound to the aniline group, as confirmed by the signal of the aliphatic protons next to the aniline at 4.15-3.99 ppm versus $3.81 \mathrm{ppm}$ for the leg in the free alcohol form. Infrared spectroscopy showed the presence of the characteristic $C \equiv O$ stretch vibrations at 2073, 2031, and $2000 \mathrm{~cm}^{-1}$ (Figure 8.2), similar to the spectrum shown for 1 (Figure 8.1).

To immobilize the tris-adamantyl hydrogenase mimic 5 on Au-2 and Si-3, the $\beta-C D$ monolayers were immersed in an aqueous solution of $10 \mu \mathrm{M}$ guest 5 with $0.5 \% \mathrm{v} / \mathrm{v}$ methanol and $1 \mathrm{mM} \beta-C D$ to enhance the solubility. The resulting immobilized host-guest complexes showed contact angle values of $59.9^{\circ} \pm 0.8,70.3^{\circ} \pm 1.2$, and $61.7^{\circ} \pm 0.7$ for Au-2.5, Si(p)-3·5, and Si $\left(\mathrm{p}^{++}\right)-3 \cdot 5$, respectively. These values are closer to each other than observed for the Au-2 and Si-3 host monolayers, as expected for monolayers of the same guest molecule. IRRAS and ATR-IR (Figure 8.2) clearly showed the presence of the $\mathrm{C} \equiv \mathrm{O}$ stretches in the $2080-2000 \mathrm{~cm}^{-1}$ region for Au-2.5 and Si-3.5 monolayers, respectively, 
which indicates that the guest molecules were still intact after immobilization. Guest immobilization was further supported by the appearance of $\mathrm{C}-\mathrm{H}$ stretches at 2918 and $2857 \mathrm{~cm}^{-1}$ for the Au-2.5 monolayer.

XPS of Au-2.5 and Si-3.5 monolayers was used to quantify the amounts of immobilized guest. The XPS spectra (Table 8.2) showed the presence of Fe atoms on all substrates, which indicates catalyst immobilization. For the Si substrates, attenuation of the Si signal and an increase of the $\mathrm{C}$ signal were observed, which denote an increase in layer thickness, i.e., adsorption of guest molecules. The I/Fe ratio on Au-2.5 substrates was used to obtain a rough estimate of the surface coverage, when assuming divalent interactions (vide infra) and not taking into account the differences in signal attenuation. This ratio equaled to 6.2 compared to the maximum value of 7.0 , which indicated a relatively high percentage of occupied host molecules. On the Si(p) substrate no $\mathrm{S}$ atoms were detectable. This is in contradiction with the detected presence of Fe in the XPS spectra and of the $\mathrm{C} \equiv \mathrm{O}$ bonds observed in the ATR-IR spectra of other $\mathrm{p}$-type $\mathrm{Si}$ substrates, which indicate that the $\mathrm{S}$ atoms should be present as the bridge between $\mathrm{Fe} / \mathrm{C} \equiv \mathrm{O}$ and the substrate. Moreover, $\mathrm{S}$ atoms were detected at the $\mathrm{Si}\left(\mathrm{p}^{++}\right)-3 \cdot 5$ substrate, which suggests that the absence of $\mathrm{S}$ atoms at the $\mathrm{Si}(\mathrm{p})$ substrate is a measurement artefact.

Table 8.2. Relative atomic percentages from XPS measurements on surfaces modified with monolayers of $\beta-C D$ and subsequently adsorbed guest 5 .

\begin{tabular}{lccccccc} 
Sample & Substrate & \multicolumn{5}{c}{ Monolayer } \\
& Au or Si (\%) & I (\%) & C (\%) & O (\%) & N (\%) & S (\%) & Fe (\%) \\
Au-2.5 & $22.3 \pm 0.6$ & $1.3 \pm 0.1$ & $53.2 \pm 1.5$ & $20.9 \pm 1.0$ & $1.42 \pm 0.57$ & $0.72 \pm 0.20$ & $0.21 \pm 0.18$ \\
Si(p)-3 & $49.1 \pm 2.0$ & & $28.5 \pm 2.2$ & $21.6 \pm 0.5$ & $0.82 \pm 0.36$ & & \\
Si(p)-3.5 & $35.9 \pm 1.3$ & $38.3 \pm 1.6$ & $24.8 \pm 0.7$ & $0.88 \pm 0.15$ & $0.00 \pm 0.00$ & $0.16 \pm 0.13$ \\
Si $\left(p^{++}\right)-3$ & $48.8 \pm 1.1$ & $28.4 \pm 1.3$ & $22.0 \pm 0.6$ & $0.80 \pm 0.13$ & & \\
Si $\left(p^{++}\right)-3 \cdot 5$ & $31.4 \pm 0.4$ & $45.3 \pm 0.3$ & $21.6 \pm 0.2$ & $1.04 \pm 0.35$ & $0.19 \pm 0.22$ & $0.72 \pm 0.14$
\end{tabular}

Similar to the XPS results of Si-1 substrates (Section 8.2.1), the relative atomic percentages for $\mathrm{N}: \mathrm{S}$ :Fe deviated from the theoretically expected ratio. The N:S:Fe ratio was expected to be 1:2:2 based on the molecular structure of guest molecule 5 , but a value of 7:3.6:1 was observed experimentally for the Au-2.5 monolayers. The $\mathrm{N}$ signal of $\mathrm{Si}\left(\mathrm{p}^{++}\right)-3.5$ involved contributions from both host 3 and guest 5 , which impeded an accurate determination of the $\mathrm{N}$ contribution of the guest molecule 5 . The $\mathrm{S}$ :Fe ratio of this Si substrate equaled to 1:3.8, in contrast to the expected 1:1 ratio. Alternatively, the outer moieties of the hydrogenase mimic, i.e., the $\mathrm{Fe} / \mathrm{C} \equiv \mathrm{O}$ groups, could be used to verify the intactness of the guest molecules, since a ratio of $1: 3$ is expected. The $C \equiv 0$ moiety was, however, not clearly distinguishable in the XPS C1s element spectra (data not shown). Its binding energy was expected at around $288.4 \mathrm{eV}$ in the $\mathrm{C} 1 \mathrm{~s}$ element 
spectrum, ${ }^{38,39}$ which overlaps with the $\mathrm{C}=\mathrm{O}$ binding energy of the heptaalkynefunctionalized $\beta-C D 3$ on $\mathrm{Si}$ and of adventitious $\mathrm{CO}_{2}$. On Au-2.5 a C $\equiv 0$ :Fe ratio of 13:1 was found, compared to the theoretical ratio of $3: 1$, which indicates that the amount of Fe measured is lower than expected and/or the deconvoluted signal also contained a contribution of adventitious $\mathrm{CO}_{2}$. In short, sensitivity issues precluded any conclusions about the intactness of the guest molecules, but the immobilization seemed to be successful.

The affinity of the host-guest interactions was assessed by surface plasmon resonance (SPR) on a Au-2 monolayer. An SPR titration was performed with increasing concentrations of guest 5 in aqueous solutions in the presence of $1 \mathrm{mM} \beta-C D$ and $0.5 \% \mathrm{v} / \mathrm{v}$ methanol. Figure 8.3 shows a titration curve similar to the binding of a divalent guest reported before, ${ }^{27}$ which can be fitted using a thermodynamic model for multivalent host-guest interactions on a surface in the presence of a competing host in solution. ${ }^{40}$ As fitting parameters, the solution affinity for $\beta-C D$ and adamantane in solution, $K_{i, l}=4.6 \times 10^{4} \mathrm{M}^{-1}$, the effective concentration, $C_{\text {eff }}=0.2 \mathrm{M}$, and the concentration of the competing $\beta-C D,\left[\beta-C D_{1}\right]=1 \mathrm{mM}$, were used to fit the binding affinity of a single $\beta-C D /$ adamantane interaction at the surface, $K_{i, s}$, as well as the maximum SPR angle change $\left(\Delta \theta_{\max }\right)$ in a least squares minimization routine. ${ }^{27}$ The guest was assumed to bind trivalently, as reported before for guests with the same tris-adamantyl moiety. ${ }^{28,29}$ This did, however, only result in proper fitting with either $K_{i, s}=5 \times 10^{3} \mathrm{M}^{-1}$ or $C_{\text {eff }}<10 \mathrm{mM}$. The lower affinity for adamantyl groups at the surface compared to binding in solution is unlikely because of the comparability to host-guest monolayers used earlier with comparable guest molecules. A lower $C_{\text {eff }}$ could be due to a host monolayer with low packing density. The best fitting was obtained when assuming effective divalent binding, which resulted in $K_{i, s}=3.3 \times 10^{4} \mathrm{M}^{-1}$ and $\Delta \theta_{\max }=0.390^{\circ}$. This value for the individual hostguest interaction at the surface is in line with the values observed before. Therefore we conclude that guest 5 binds in a divalent fashion. This is, however, not in line with the binding of tris-adamantyl guests described before. ${ }^{28,29}$ Theoretically upside down binding of the guest is possible, since inclusion of the [FeFe] core into $\beta-C D$ has been reported in the literature. ${ }^{36}$ This is, however, not expected to play a role here since the

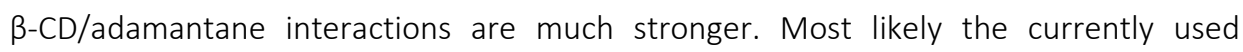
heptaiodo-functionalized $\beta-C D$ monolayer contained an average spacing that permits divalent but prohibits trivalent binding. The $\beta-C D$ monolayers used before for trivalent guest binding were made using heptathioether-functionalized $\beta-C D$ on gold. These monolayers showed a higher packing density than the Au-2 monolayer used here, i.e., $5.9 \times 10^{-11} \mathrm{~mol} / \mathrm{cm}^{2}$ versus $3.8 \times 10^{-11} \mathrm{~mol} / \mathrm{cm}^{2}$, as discussed above using Au-2.4 monolayers. The lower packing density is expected to result in the observed divalent binding. 


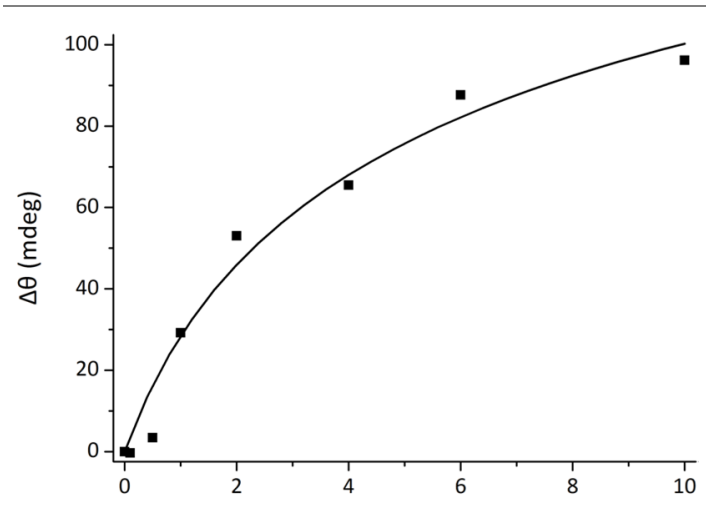

[5] $(\mu \mathrm{M})$

Figure 8.3. SPR titration of guest 5 on a Au-2 substrate in the presence of $1 \mathrm{mM} \beta-C D$, where the solid line is a fit to a thermodynamic multivalent model.

\subsubsection{Redox activity and catalysis}

Electrochemistry of supramolecular guest 5 in solution indicated that the hydrogenase mimic was still redox active after synthesis (Figure 8.4). The catalyst was dissolved in an aqueous solution with $10 \mathrm{mM} \beta-\mathrm{CD}$, which showed an electrochemical response in cyclic voltammograms at $\mathrm{pH} 4.0$ (Figure 8.4a). The observed reduction peaks could originate from either catalyst or proton reduction. ${ }^{34} \mathrm{CV}$ of a solution without 5 did not show peaks in this region, which confirmed that the observed peaks originate from the hydrogenase mimic 5. Differential pulse voltammetry (DPV), a more accurate technique than CV, was used to reveal more peaks, including the small peak around $-0.85 \mathrm{~V}$ observed in Figure 8.4a. In DPV (Figure 8.4b), the first reduction peak was observed at $-0.85 \mathrm{~V}$ vs. $\mathrm{AgCl}$, $-0.93 \mathrm{~V},-0.97 \mathrm{~V}$, and $-0.99 \mathrm{~V}$ at $\mathrm{pH} 4.0,6.0,7.4$, and 8.0, respectively. The shift in the electrochemical activity when measuring at different $\mathrm{pHs}$ indicates that the electrochemistry is coupled to a protonation step. ${ }^{13} \mathrm{~A}$ plot of the peak potential versus $\mathrm{pH}$ gave a slope of $-35 \mathrm{mV}$, which is close to the theoretical value of $-30 \mathrm{mV}(-2.3 R T / 2 \mathrm{~F})$ for a two-electron process. This indicates that the transfer of one proton is coupled to the transfer of two electrons, as has been observed before for other hydrogenase mimics. ${ }^{13,15}$ The increase in current at more negative potentials could be a sign of catalytic proton reduction, of which both the onset potential and the height of the current are (highly) $\mathrm{pH}$ dependent. The large (negative) current increase observed in Figure 8.4a might also be due to proton reduction, with an onset potential around $-1.0 \mathrm{~V} v \mathrm{~s}$. $\mathrm{Ag} / \mathrm{AgCl}$ at $\mathrm{pH}$ 4.0. Proton reduction occurs at milder potentials when the $\mathrm{pH}$ is lower and vice versa, so for this reaction it is advantageous to test the system at lower $\mathrm{pH}$. 

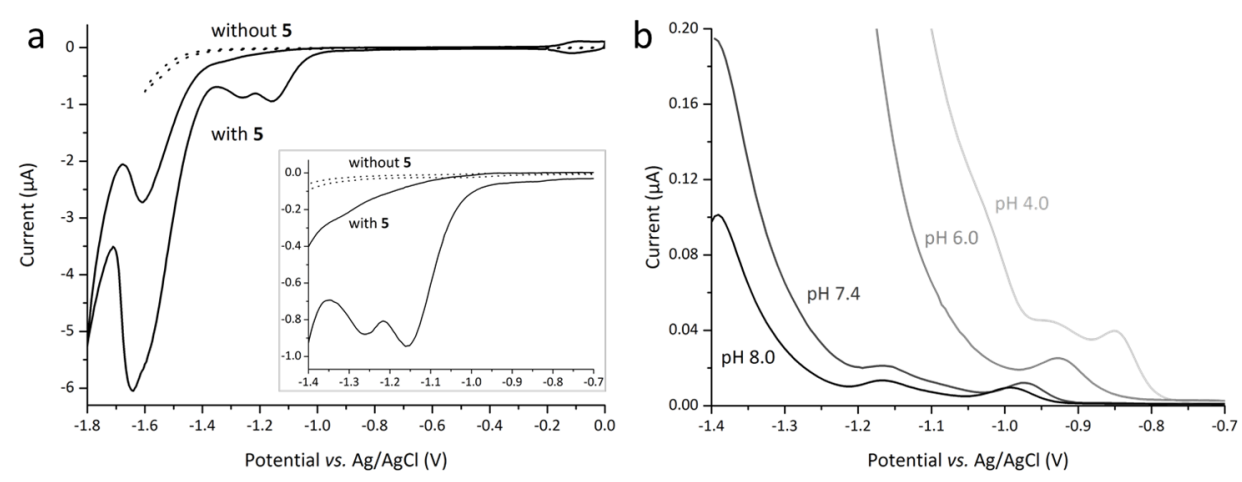

Figure 8.4. Electrochemistry of $<10 \mu \mathrm{M} 5$ in an aqueous $100 \mathrm{mM} \mathrm{Na} \mathrm{H}_{y} \mathrm{PO}_{4}$ buffer with $10 \mathrm{mM} \beta-\mathrm{CD}$ using a) $\mathrm{CV}$ at $\mathrm{pH} 4.0(2 \mathrm{~V} / \mathrm{s})$, compared to the solution without 5 , where the inset shows a zoom-in at the -0.7 to $-1.4 \mathrm{~V}$ region and b) DPV at different $\mathrm{pHs}$.

Subsequently, electrochemistry was used to study the redox activity of the immobilized hydrogenase mimics. Specifically, CV was applied to the functionalized gold and silicon electrodes in an aqueous solution at $\mathrm{pH}$ 7.0. At this $\mathrm{pH}$, reduction of a solution with catalyst 5 could be detected by DPV at around -0.9/-1.0 V vs. Ag/AgCl (Figure 8.4b). For the covalently bound hydrogenase mimic, however, CV of Si(p)-1 did not reveal any obvious difference between functionalized and unfunctionalized substrates (Figure 8.5a). Nonetheless, for $\mathrm{p}^{++}$substrates the very first scans showed a (negative) increase in current for $\mathrm{Si}\left(\mathrm{p}^{++}\right)-1$ compared to a bare $\mathrm{p}^{++}$substrate (Figure $8.5 \mathrm{~b}$ ), with a small peak around $-0.95 \mathrm{~V}$ vs. $\mathrm{Ag} / \mathrm{AgCl}$ as expected. This peak disappeared after a few cycles, which indicates desorption or destruction of the redox-active molecules. This is supported by a decrease in contact angle from $>75^{\circ}$ after hydrosilylation to $<40^{\circ}$ after electrochemistry and a decrease in layer thickness from $>7 \mathrm{~nm}$ to $<3.5 \mathrm{~nm}$, as measured by ellipsometry. These results were not reproducible, since other cyclic voltammograms did not show a difference in peaks between Si-1 and bare Si substrates. Also illumination (420 nm LED) did not elucidate other reduction events, whereas illumination has been used for lightassisted reduction on $\mathrm{p}$-type $\mathrm{Si}^{34}$ Hypothetically the multilayer of 1 (Section 8.2.1) could be too thick to be measured by electrochemistry or the catalyst could have been deactivated during the hydrosilylation reaction. 

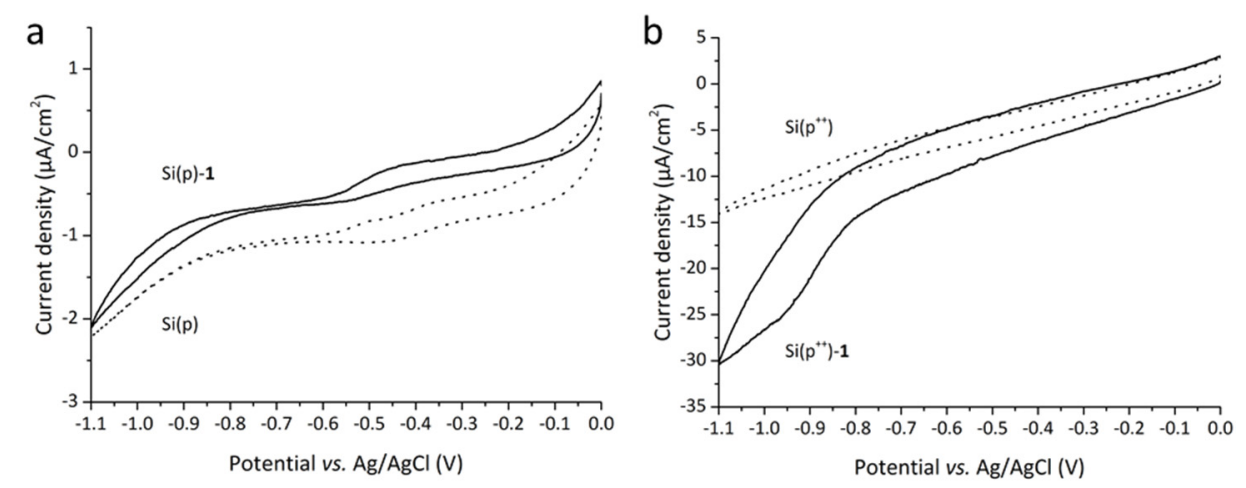

Figure 8.5. First cyclic voltammograms at $0.1 \mathrm{~V} / \mathrm{s}$ in an aqueous phosphate-buffered saline (PBS) solution of $\mathrm{pH} 7.0$ at bare $\mathrm{Si}$ and $\mathrm{Si}-1$ substrates measured in the dark for a) $\mathrm{p}$-type and b) $\mathrm{p}^{++} \mathrm{Si}$.

Similarly, electrochemistry was employed to detect reduction of the supramolecularly immobilized hydrogenase mimic 5 on $\beta-C D$ monolayers. CV of Au-2.5 and Si-3.5 substrates at $\mathrm{pH} 7.0 \mathrm{did}$, however, not show peaks at around -0.9/-1.0 vs. $\mathrm{Ag} / \mathrm{AgCl}$, i.e., no electrochemical response of the catalyst could be detected (Figure 8.6). Au-2 and bare Si $\mathrm{p}^{++}$substrates did show some redox peaks in the potential range measured 0.0 to $-1.1 \mathrm{~V} v \mathrm{vs} . \mathrm{Ag} / \mathrm{AgCl}$ ), which could be due to, for example, reactions of the substrate with water, reactions of the electrolyte, or adsorption/readsorption of the heptaiodo legs of the $\beta-C D$ derivative on gold. These peaks were not assigned to a specific redox event, since any catalytic activity of the guest should have been confirmed by a difference between the graphs with and without guest. The other conditions tested included a wider potential range (down to $-1.5 \mathrm{~V}$ vs. $\mathrm{Ag} / \mathrm{AgCl}$ ), $\mathrm{p}$-type instead of $\mathrm{p}^{++} \mathrm{Si}$ substrates, higher scan rates (up to $5 \mathrm{~V} / \mathrm{s}$ ), $\mathrm{pH}$ 6.0, DPV instead of $\mathrm{CV}$, and illumination of the Si samples (420 nm LED), but none of these revealed any reduction event.
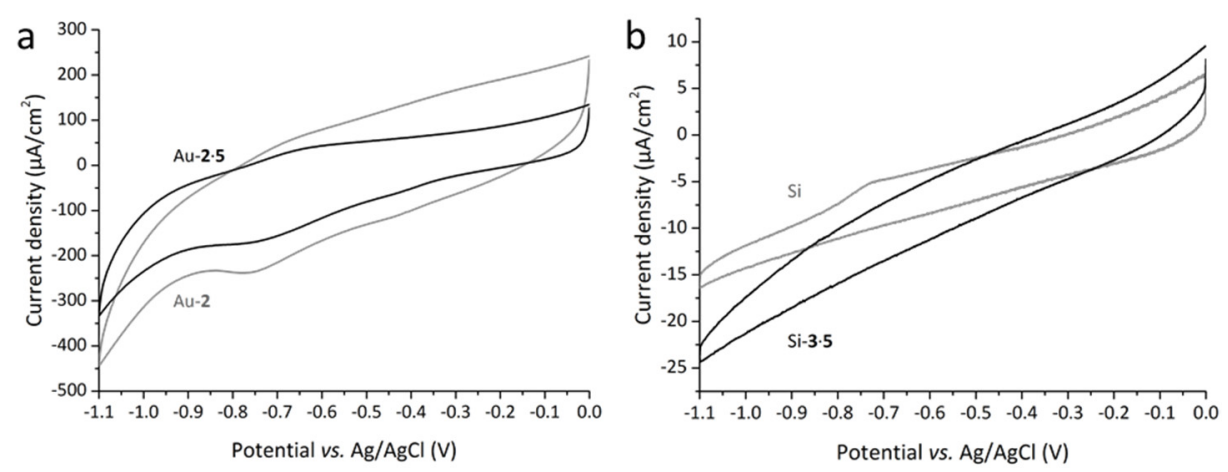

Figure 8.6. Cyclic voltammetry at $1.0 \mathrm{~V} / \mathrm{s}$ in an aqueous phosphate-buffered saline (PBS) solution of $\mathrm{pH} 7.0$ at a) Au- 2 and Au-2.5 substrates and b) bare Si( $\left.\mathrm{p}^{++}\right)$and $\mathrm{Si}\left(\mathrm{p}^{++}\right)-3.5$ substrates, measured in the dark. 
Another method to study the catalytic activity involves the addition of an acid to induce proton reduction in an organic solvent. ${ }^{12,41,42}$ On surfaces with the covalently bound hydrogenase mimic (Si-1), cyclic voltammetry was performed in acetonitrile with $0.1 \mathrm{M}$ tetrabutylammonium hexafluorophosphate $\left(\mathrm{TBAPF}_{6}\right)$ as electrolyte, $1 \mathrm{mM}$ ferrocenemethanol as internal standard, and 0, 1, 2, 3, or 4 mM acetic acid, under illumination with a $420 \mathrm{~nm}$ LED. On Si(p)-1 this resulted in a clear reduction for 1 and $2 \mathrm{mM}$ acetic acid, whereas a control on bare Si(p) did not show these peaks (Figure 8.7). The peak current roughly doubled when switching from 1 to $2 \mathrm{mM}$ acetic acid, which corresponds to observations reported before where the peak intensity increased linearly with the amount of acid added. ${ }^{41,42}$ These results indicate that the catalyst at the surface is still catalytically active. For higher acetic acid concentrations ( 3 and $4 \mathrm{mM}$ ), however, the cyclic voltammograms were equal to those of $0 \mathrm{mM}$ acetic acid or bare $\mathrm{Si}(\mathrm{p})$. The catalyst seemed to be deactivated or removed from the surface. Rapid inactivation has been observed before for a comparable catalyst complex, ${ }^{8}$ which was attributed to elimination of the carbonyl ligands from the [FeFe] center. Hypothetically, a milder light source could be used to avoid catalyst destruction, ${ }^{43}$ but experiments with a $617 \mathrm{~nm}$ LED did not show any catalytic activity (data not shown). The preliminary results with the $420 \mathrm{~nm}$ LED source were not reproducible either, so more research is needed to elucidate the exact catalytic activity of Si-1 substrates.

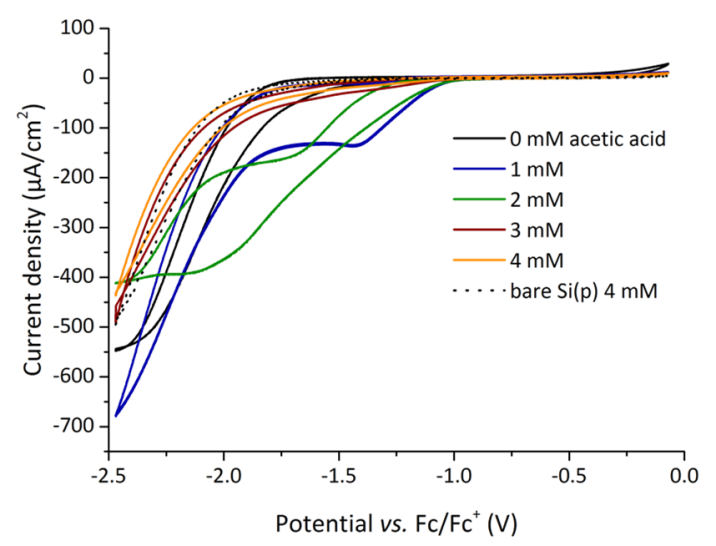

Figure 8.7. Cyclic voltammetry at $0.1 \mathrm{~V} / \mathrm{s}$ in acetonitrile with $0.1 \mathrm{M} \mathrm{TBAPF}_{6}$ with $0,1,2,3$, and $4 \mathrm{mM}$ acetic acid at Si(p)-1, measured under $420 \mathrm{~nm}$ illumination. The cyclic voltammogram of the $4 \mathrm{mM}$ acetic acid solution at bare $\mathrm{Si}(\mathrm{p})$ is added for comparison.

The tests with acetic acid in acetonitrile, as described above for Si-1 substrates, could not be performed on the supramolecularly immobilized hydrogenase mimics (5), since the $\beta-C D$ monolayers are not compatible with organic solvents. Instead, proton reduction on $\mathrm{Au}-2.5$ and $\mathrm{Si}-3.5$ substrates was tested in aqueous solutions at low $\mathrm{pH}$, since proton reduction occurs at milder potentials when the $\mathrm{pH}$ is lower and vice versa (Figure 8.4b). 
Therefore, $\mathrm{CV}$ in solutions of $\mathrm{pH} 3.0$ and 4.0 was tested until $-2.0 \mathrm{~V}$ vs. $\mathrm{Ag} / \mathrm{AgCl}$, even though the stability of the monolayers was tested neither for these $\mathrm{pHs}$ nor in this potential range. At pH 4.0, the onset potential of catalysis was observed around $-1.0 \mathrm{~V} v \mathrm{vs}$. $\mathrm{Ag} / \mathrm{AgCl}$ for the catalyst in solution (Figure 8.4). The onset of the background reaction of the Au-2 substrates without guest was, however, at a more positive potential than $-1.0 \mathrm{~V}$ vs. $\mathrm{Ag} / \mathrm{AgCl}$, as shown in Figure 8.8a for $\mathrm{pH}$ 3.0. The other conditions tested, i.e., $\mathrm{pH} 4.0$ and 6.0, and DPV instead of CV, showed the same results, which made it impossible to visualize any difference between the Au-2 and Au-2.5 substrates. Because of the high overpotential needed for the catalyst, the gold substrates started proton reduction at an earlier stage than the catalyst. For silicon substrates, however, the background reaction tended to start at more negative potentials. The best result obtained for these substrates showed a steeper decrease in current for the $\mathrm{Si}\left(\mathrm{p}^{++}\right)-3.5$ substrates with catalyst, including a tiny peak at $-1.32 \mathrm{~V} v \mathrm{~s}$. $\mathrm{Ag} / \mathrm{AgCl}$ with a peak current density of about $3 \mu \mathrm{A} / \mathrm{cm}^{2}$ (Figure $8.8 b)$. The crossing of the anodic and cathodic signal could denote an electrochemically induced reaction at the surface. ${ }^{44,45}$ This preliminary result might indicate catalyst activity but was not reproducible and disappeared after two cycles. All other conditions tested, i.e., p-type instead of $\mathrm{p}^{++} \mathrm{Si}$ substrates, $\mathrm{pH} 4.0$ and 6.0, DPV instead of CV, higher scan rates (up to $5 \mathrm{~V} / \mathrm{s}$ ), and illumination of the Si samples (420 nm or $617 \mathrm{~nm}$ LED) showed graphs with similar shapes for bare Si or Si-3.5. The current density sometimes differed between the two substrates, but this was randomly in favor of either of the two substrates instead of a consistently higher current for the Si-3.5 samples as expected. Nonetheless, the silicon substrates seem to be preferred over gold substrates since the overpotential of the bare substrate was higher than that of the catalyst.
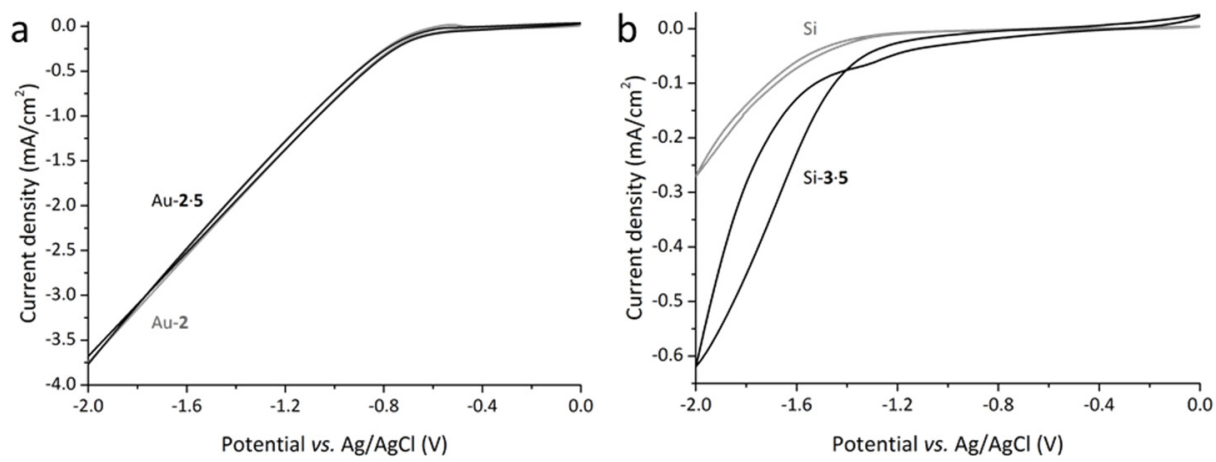

Figure 8.8. Cyclic voltammetry at $0.2 \mathrm{~V} / \mathrm{s}$ in an aqueous $100 \mathrm{mM} \mathrm{Na}_{x} \mathrm{H}_{y} \mathrm{PO}_{4}$ buffer of $\mathrm{pH} 3.0$ at a) Au2 and $\mathrm{Au}-2.5$ substrates and $\mathrm{b})$ bare $\mathrm{Si}\left(\mathrm{p}^{++}\right)$and $\mathrm{Si}\left(\mathrm{p}^{++}\right)-3.5$ substrates, measured in the dark.

\subsection{Conclusions and outlook}

All in all, a [FeFe]hydrogenase mimic has been immobilized as proton reduction catalyst by two routes, i.e., either covalently on silicon substrates or by host-guest interactions at 
$\beta-C D$ monolayers on gold and silicon substrates. For both routes, the characteristic $C \equiv 0$ stretch vibrations of the hydrogenase mimic headgroup and the presence of $\mathrm{N}, \mathrm{S}$, and Fe was confirmed by infrared spectroscopy and XPS, respectively. The covalently bound hydrogenase mimic appeared to form multilayers instead of a monolayer, as shown by ellipsometry measurements. The supramolecular hydrogenase mimic was synthesized with a tris-adamantyl moiety to ensure strong anchoring. A non-close-packed $\beta-C D$ monolayer resulted, however, in divalent host-guest interactions according to SPR on gold substrates.

Electrochemistry was employed to test the catalytic properties of the immobilized catalyst. Only preliminary results have shown catalyst or proton reduction at the gold and silicon surfaces. The covalently bound hydrogenase mimic showed reduction on $\mathrm{p}^{++} \mathrm{Si}$ at a potential as expected from measurements in solution. This peak, however, disappeared after a few cycles, which denotes desorption or destruction of the catalyst. For the supramolecularly bound hydrogenase mimic, no reduction could be detected at all. Proton reduction was tested with acetic acid solutions on the covalently bound catalyst. This resulted on $\mathrm{p}$-type $\mathrm{Si}$ in a clear reduction signal when using 1 and $2 \mathrm{mM}$ acetic acid solutions. For higher acetic acid concentrations, no reduction was observed anymore, which indicates catalyst deactivation or removal from the surface. When testing proton reduction on $\beta-C D$ monolayers, a large background signal was observed from the bare substrates. Silicon substrates seemed to be the most suitable for this purpose, where a preliminary result showed a small catalytic peak and a steeper decrease in current compared to a bare substrate. Thus, immobilization of the catalyst was successful on both gold and silicon substrates, but further research is needed to confirm its catalytic activity.

To elucidate whether the catalyst still contained its catalytic function after immobilization on gold substrates, SPR and CV could potentially be combined. ${ }^{46}$ This joint technique would visualize the direct relation between a change in electrochemical response and immobilization/desorption of the (supramolecular) catalyst. For both immobilization routes, the amount of hydrogen produced while applying a constant potential could be detected by gas chromatography (GC), for example, although the background signal from reduction of protons at the substrate should then be minimized as much as possible. Theoretically, a hydrogen production rate of $75 \mu \mathrm{L} /\left(\mathrm{cm}^{2} \cdot \mathrm{h}\right)$ could be achieved, when assuming a maximum catalyst coverage of $1.7 \times 10^{-11} \mathrm{~mol} / \mathrm{cm}^{2}$ (based on the supramolecular route) and a turnover frequency (TOF) of $100 \mathrm{~s}^{-1}$, which is fairly underestimated for a worst case scenario. ${ }^{9}$ Using a $5 \mathrm{~mL}$ argon carrier gas flow, this would result in about $250 \mathrm{ppm} \mathrm{H}_{2}$ which is far above the detection limit of GC. If needed, a 3D topography could be added to the Si surfaces, for example in the form of silicon nano/micropillars, to increase the amount of catalyst per sample area and thus achieve a higher $\mathrm{H}_{2}$ production. 
Alternatively, the systems could be designed differently, for example by adapting the catalyst towards one with a lower overpotential. Considering the substrates used, the silicon electrodes seemed to be the most suitable, since the gold substrates interfered with a large background signal. The latter might be solved by using an electrode of mercury and gold (gold amalgam) instead, for which the overpotential should be higher whereas monolayer formation should still be possible. ${ }^{47}$

The two catalyst immobilization routes have their own advantages and disadvantages. Whereas covalent immobilization results in a higher catalyst loading at the surface due to multilayer formation, the molecules have to withstand the harsh conditions of the hydrosilylation reaction. The supramolecular catalyst binding is weaker due to the divalent interactions, which could induce guest desorption over time. The host-guest interactions could, however, also be used to reload the $\beta$-CD monolayer with new catalyst when desired, i.e., by incubation with a fresh guest solution after desorption of the aged guest by competition with free $\beta-C D$ in solution. ${ }^{27}$ This would make both the covalent and noncovalent immobilization routes suitable for the production of a solar-to-fuel device.

\subsection{Acknowledgments}

This work is the result of a joint research project with the University of Amsterdam (Homogeneous, Supramolecular and Bio-inspired Catalysis group, prof. J.N.H. Reek). René Becker (UvA) is gratefully acknowledged for the synthesis of the alkyne-functionalized hydrogenase mimic 1, the initial electrochemistry experiments on Si-1 substrates, proofreading of the manuscript, and for fruitful discussions. Richard Egberink and Alejandro Méndez-Ardoy are kindly thanked for the synthesis of the heptaiodofunctionalized $\beta-C D$ compound 2. Alejandro Méndez-Ardoy is also gratefully acknowledged for the syntheses of heptaalkyne-functionalized $\beta-C D$ compound 3 and bisadamantyl ferrocene guest 4. Esther Schippers (UvA) and Riccardo Zaffaroni (UvA) are gratefully acknowledged for the synthesis of tris-adamantyl hydrogenase mimic 5, fruitful discussions on the electrochemistry experiments, and proofreading of the manuscript. Esther Schippers is also thanked for the characterization and electrochemistry experiments of 5 in solution.

\subsection{Experimental section}

\subsubsection{Materials}

Glass-supported gold substrates (2.54 cm diameter, $2 \mathrm{~nm}$ Ti adhesion layer, $50 \mathrm{~nm}$ Au for SPR or $200 \mathrm{~nm}$ Au for electrochemistry and IRRAS) were obtained from Ssens (Enschede, the Netherlands). Silicon wafers (<100>-oriented, $100 \mathrm{~mm}$ diameter, single side polished) were obtained from Okmetic (Finland) as p-type (boron, resistivity 5-10 $\Omega \cdot \mathrm{cm}$ ) or $\mathrm{p}^{++}$ 
(boron, 0.010-0.025 $\Omega \cdot \mathrm{cm}$ ). For ATR-IR, double side polished p-type silicon wafers were used with parameters equal to the single side polished p-type wafers. Reagents and solvents were obtained from commercial sources and used without further purification unless stated otherwise. Dichloromethane used for the synthesis of 1 was distilled over $\mathrm{CaH}_{2}$ before use. 1-[Bis(dimethylamino)methylene]-1H-1,2,3-triazolo[4,5-b]pyridinium 3oxide hexafluorophosphate (HATU) was obtained from GenScript. The solvents used for the synthesis of 5 (triethylamine, tetrahydrofuran (THF), and acetonitrile) were freshly distilled. Heptakis(2,3-di-O-acetyl-6-deoxy-6-iodo)-cyclomaltoheptaose (2, heptaiodofunctionalized $\beta-C D)^{48}$ and the trivalent ferrocene-containing guest $4^{37}$ were synthesized according to literature procedures. Heptakis-6-deoxy-6-(undec-10-ynamido)cyclomaltoheptaose (3, heptaalkyne-functionalized $\beta-C D$ ) was synthesized as described in Chapter 7. Milli-Q water with a resistivity $>18 \mathrm{M} \Omega \cdot \mathrm{cm}$ was obtained from a Milli-Q Integral water purification system (Merck Millipore). Glassware used for the hydrosilylation reactions was dried overnight at $120^{\circ} \mathrm{C}$.

\subsubsection{Methods}

Synthesis of alkyne-functionalized hydrogenase mimic 1 . The synthesis of 1 was carried out under a nitrogen atmosphere using general Schlenk techniques. $\mathrm{Fe}_{2}(\mu$-mcbdt)(CO) 6 (mcbdt = 1-monocarboxylbenzene-2,3-dithiolate) was synthesized according to a procedure from the literature, ${ }^{31}$ where it was isolated as an uncharacterized sideproduct. To a $50 \mathrm{~mL}$ Schlenk flask was added $\mathrm{Fe}_{2}(\mu$-mcbdt)(CO) 6 (93 mg, $0.2 \mathrm{mmol}$ ), $N, N^{\prime}$-diisopropylcarbodiimide $(35 \mu \mathrm{L}, 1.1 \mathrm{eq})$ and propargylamine $(1.1 \mathrm{eq})$ in dichloromethane $(10 \mathrm{~mL})$. The solution was stirred for $30 \mathrm{~min}$ at room temperature, preadsorbed on silica and purified by column chromatography in air using nondegassed solvents ( $1 \%$ methanol in dichloromethane). The desired product eluted as an orange band, and the product after solvent removal was stored in the dark at $4{ }^{\circ} \mathrm{C}$ until use. ${ }^{1} \mathrm{H}$ NMR (400 MHz, dichloromethane- $d_{2}$, Figure 8.9) $\delta 7.27(\mathrm{dd}, J=7.5,1.2 \mathrm{~Hz}, 1 \mathrm{H}), 6.99$ $(\mathrm{dd}, J=7.8,1.2 \mathrm{~Hz}, 1 \mathrm{H}), 6.74(\mathrm{t}, J=7.7 \mathrm{~Hz}, 1 \mathrm{H}), 6.36(\mathrm{bs}, 1 \mathrm{H}), 4.18(\mathrm{dd}, J=5.4,2.6 \mathrm{~Hz}, 2 \mathrm{H})$, $2.33(\mathrm{t}, J=2.6 \mathrm{~Hz}, 1 \mathrm{H})$. IR $v_{C O} 2080,2043,2001 \mathrm{~cm}^{-1}$. 


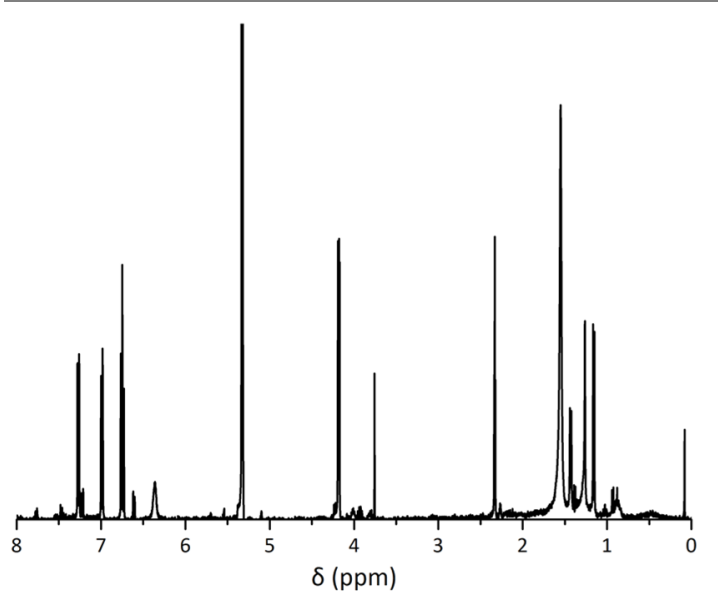

Figure 8.9. ${ }^{1} \mathrm{H}$ NMR spectrum of 1 (measured at $400 \mathrm{MHz}$ in dichloromethane- $d_{2}$ ).

Synthesis of tris-adamantyl hydrogenase mimic 5. $\mathrm{Fe}_{2}\left(\mu\right.$-mcbdt) $(\mathrm{CO})_{6}{ }^{31}$ and 3,4,5-tris [2-(2\{2-[2-(adamantan-1-yloxy)ethoxy]ethoxy\}ethoxy)-ethoxy]phenylamine (a trivalent adamantane guest with amino headgroup, $\left.\mathrm{Ad}_{3}-\mathrm{NH}_{2}\right)^{29}$ were synthesized according to literature procedures. A solution of $\mathrm{Fe}_{2}\left(\mu\right.$-mcbdt) $(\mathrm{CO})_{6}(21 \mathrm{mg}, 0.045 \mathrm{mmol}), \mathrm{Ad}_{3}-\mathrm{NH}_{2}$ (40 mg, $0.04 \mathrm{mmol}), \mathrm{HATU}(22 \mathrm{mg}, 0.052 \mathrm{mmol})$, and triethylamine $(34 \mu \mathrm{L}, 0.2 \mathrm{mmol})$ in $5 \mathrm{~mL} \mathrm{2:3}$ acetonitrile/THF was stirred under argon atmosphere at $50{ }^{\circ} \mathrm{C}$. The reaction was followed with thin-layer chromatography (eluent: $5 \%$ triethylamine in ethyl acetate (EtOAc)). After $30 \mathrm{~min}$ the reaction mixture was diluted with $60 \mathrm{~mL}$ EtOAc and washed with solutions of $1 \mathrm{M} \mathrm{HCl}(3 \times 20 \mathrm{~mL})$, saturated $\mathrm{NaHCO}_{3}(3 \times 20 \mathrm{~mL})$, and saturated $\mathrm{NaCl}$. Subsequently, the organic layer was dried on $\mathrm{MgSO}_{4}$ and the volatiles were evaporated using a rotary evaporator. Column chromatography (5-10\% triethylamine in EtOAc) afforded 5 as an orange oil ( $9 \mathrm{mg}, 0.006 \mathrm{mmol}, 15 \%$ yield), which was stored in the dark at $4{ }^{\circ} \mathrm{C}$ until use. ${ }^{1} \mathrm{H}$ NMR $\left(400 \mathrm{MHz}\right.$, acetonitrile- $d_{3}$, Figure 8.10) $\delta 8.59(\mathrm{~s}, 1 \mathrm{H}), 7.33(\mathrm{~d}$, $J=7.6 \mathrm{~Hz}, 1 \mathrm{H}), 7.05(\mathrm{~s}, 2 \mathrm{H}), 6.99(\mathrm{~d}, J=7.9 \mathrm{~Hz}, 1 \mathrm{H}), 6.82(\mathrm{t}, J=7.7 \mathrm{~Hz}, 1 \mathrm{H}), 4.15-3.99(\mathrm{~m}$, $6 \mathrm{H}), 3.82-3.75(\mathrm{~m}, 4 \mathrm{H}), 3.73-3.69(\mathrm{~m}, 2 \mathrm{H}), 3.68-3.36(\mathrm{~m}, 36 \mathrm{H}), 2.11-2.06(\mathrm{~m}, 9 \mathrm{H})$, 1.73-1.66 (m, 18H), 1.65-1.58 (m, 18H). IR $v_{C O} 2073,2031,2000 \mathrm{~cm}^{-1}$. Mass spectrometry $\mathrm{CSI}+(\mathrm{m} / \mathrm{z})$ calculated $1540.47[\mathrm{M}+\mathrm{Na}]^{+}$, found 1540.47 . 


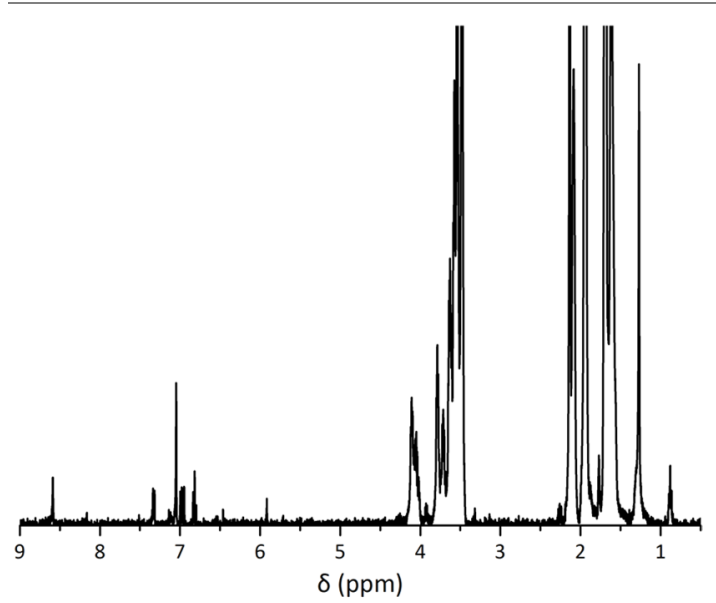

Figure 8.10. ${ }^{1} \mathrm{H}$ NMR spectrum of 5 (measured at $400 \mathrm{MHz}$ in acetonitrile- $d_{3}$ ).

Hydrosilylation of 1 on silicon. The hydrosilylation of 1 onto $\mathrm{Si}-\mathrm{H}$, including the preparation and cleaning steps, was performed in the dark using aluminum foil. The reactant solution, a $2 \mathrm{mM}$ solution of alkyne-functionalized hydrogenase mimic 1 in toluene, was degassed by four freeze-pump-thaw cycles. More than $10 \mathrm{~mL}$ solution was used to avoid solvent evaporation overnight. Silicon $\mathrm{p}$ and $\mathrm{p}^{++}$substrates were diced into pieces of $2 \times 2 \mathrm{~cm}^{2}$ and sonicated in acetone for $10 \mathrm{~min}$ to remove particles generated during dicing. A hydrogen-terminated surface was created by 2 min immersion in an aqueous $1 \% \mathrm{HF}$ solution. For electrochemistry, an Ohmic contact was created at the back side by sputtering a 1 um aluminum/silicon layer (99/1\% Al/Si, Oxford PL400, 7 kW). Afterwards, the substrates were immediately transferred into a nitrogen glovebox and immersed in the degassed solution of 1 . The reaction flask was equipped with a capillary as a nitrogen inlet and a reflux condenser. The hydrosilylation reaction was performed overnight under continuous nitrogen flow at $80^{\circ} \mathrm{C}$. Afterwards, the wafers were cleaned by ultrasonication for $2 \mathrm{~min}$ in consecutively toluene, dichloromethane, and acetonitrile, and subsequently dried in a stream of nitrogen. The samples were characterized immediately or stored in the dark under nitrogen until use.

$\beta-C D$ monolayer formation on gold. Monolayers of heptaiodo-functionalized $\beta-C D$ were formed on gold as reported before. ${ }^{23}$ In short, substrates with gold (200 nm for electrochemistry, $50 \mathrm{~nm}$ for SPR) were cleaned by 10 min oxygen plasma (SPI Plasma Prep II, $40 \mathrm{~mA}$ ) and $20 \mathrm{~min}$ immersion in ethanol. Monolayers were formed by overnight immersion in a $0.1 \mathrm{mM}$ solution of heptaiodo-functionalized $\beta$-CD in THF:methanol (ratio $4: 1 \mathrm{v} / \mathrm{v}$ ) at room temperature under an argon atmosphere. The substrates were cleaned by rinsing with THF/methanol, ethanol, and Milli-Q water, and dried using a stream of nitrogen. 
$\beta$-CD monolayer formation on silicon. Monolayers of Si-3 were prepared as described in Chapter 7.

Guest immobilization. For guest 4, a $10 \mu \mathrm{M}$ solution was made by first dissolving the guest in methanol, followed by dilution with water to obtain a solution with $1 \% \mathrm{v} / \mathrm{v}$ methanol. A $10 \mu \mathrm{M}$ solution of guest 5 was prepared similarly but was diluted with an aqueous $\beta-C D$ solution to obtain a solution with $0.5 \% \mathrm{v} / \mathrm{v}$ methanol and $1 \mathrm{mM} \beta-C D$. The solutions of guest 5 were protected from light and used within a few hours to avoid deterioration of the compound. Guest immobilization of both guests was achieved by immersing the hostfunctionalized surfaces in the guest-containing solution for $10 \mathrm{~min}$. The surfaces were rinsed with Milli-Q water, $2 \mathrm{~mL}$ of $1 \mathrm{mM}$ aqueous $\beta-C D$ solution, and Milli- $\mathrm{Q}$ water, and dried in a stream of nitrogen. The samples were characterized immediately or stored in the dark under nitrogen until use.

\subsubsection{Equipment}

Characterization after synthesis. ${ }^{1} \mathrm{H}$ NMR spectroscopy was measured on a Bruker AV400 spectrometer. FT-IR spectroscopy was performed on a Nicolet 6700 FT-IR $\left(4 \mathrm{~cm}^{-1}\right.$ resolution, 128 scans). A high resolution mass spectrum of 5 was recorded on a JEOL JMST100LP AccuTOF LC mass spectrometer using cold spray ionization (CSI).

Contact angle measurements. Static contact angles were measured with Milli-Q water on a Krüss G10 Contact Angle Measuring Instrument equipped with a CCD camera and drop shape analysis software. Contact angles were measured directly after the monolayer formation or guest immobilization and averaged over four drops.

Attenuated total reflection infrared spectroscopy. ATR-IR spectra were measured on double side polished silicon wafer pieces treated similarly to the electrochemistry samples but lacking the aluminum/silicon contact. Spectra were collected with a Bruker spectrometer (Vertex 70v) equipped with an attenuated total reflection accessory $\left(L=15 \mathrm{~mm}\right.$ ) and MCT detector. The spectra were recorded with a resolution of $4 \mathrm{~cm}^{-1}$ and 1024 scans and treated with a spline baseline correction. Each spectrum was referenced to a hydrogen-terminated silicon sample.

Infrared reflection-absorption spectroscopy. IRRAS measurements of functionalized substrates (200 nm Au) were performed on a Nicolet 6700 FT-IR with a TOM optical module (Thermo Scientific) equipped with a photoelastic modulator (PEM, Hinds Instruments). Spectra were recorded with the $p$-polarized light incidence at $81^{\circ}$ relative to the surface normal, with the PEM wavenumber set to $2900 \mathrm{~cm}^{-1}$. The spectra were recorded with a resolution of $4 \mathrm{~cm}^{-1}$ and 128 scans. 
X-ray photoelectron spectroscopy. XPS measurements were performed on a Quantera SXM setup from Physical Electronics equipped with an Al Ka X-ray source (1486.6 eV). A detector angle of $45^{\circ}$ was used, and collected spectra were calibrated on the C1s peak at $284.8 \mathrm{eV}$. The atomic percentage of $\mathrm{S}$ was determined by multiplying the ratio between the S2s and C1s peak by the determined atomic percentage of $C$, since the S2p peak overlapped with the Si2s shake-up peak and could thus not be quantified directly. The XPS spectrum of physisorbed molecules of 1 was recorded on a Si sample onto which a $2 \mathrm{mM}$ solution of 1 in toluene was added, and the toluene was evaporated in a few hours.

Ellipsometry. Layer thicknesses on silicon substrates were measured using a Woollam M-2000Ul ellipsometer. The ellipsometric spectra were obtained at an angle of $75^{\circ}$ and fitted with a Cauchy layer with a refractive index of 1.46 .

Surface plasmon resonance spectroscopy. SPR measurements were conducted on substrates ( $50 \mathrm{~nm}$ gold) functionalized with a $\beta$-CD host monolayer using a two-channel vibrating mirror angle scan setup from Resonant Probes $\mathrm{GmbH}$. Light from a $10 \mathrm{~mW}$ HeNe laser $(\lambda=632.8 \mathrm{~nm})$ is directed onto a prism surface by a vibrating mirror. The gold substrate with the monolayer was optically matched to a high index prism using an index matching oil (Cargille, series $B, n_{D}{ }^{25}=1.700 \pm 0.002$ ). A Teflon cell was placed on the monolayer with an O-ring to avoid leakage. A syringe pump was used to flow guest solutions with different concentrations $(0.1,0.5,1,2,4,6$, and $10 \mu \mathrm{M})$ over the $\beta$-CDfunctionalized surface with a continuous flow of $100 \mu \mathrm{L} / \mathrm{min}$. The angle change was obtained when no further enhancement in angle was observed, after which a higher guest concentration was added. All guest solutions were prepared in an aqueous solution of $1 \mathrm{mM} \beta-C D$ and $0.5 \% \mathrm{v} / \mathrm{v}$ methanol, which was also used to record the baseline.

Electrochemistry. Electrochemical measurements were performed on a $\mathrm{CH}$ Instruments bipotentiostat 760D. All solutions used for electrochemical measurements were deaerated by bubbling with nitrogen for at least $10 \mathrm{~min}$. The electrochemistry experiments with guest 5 in solution were performed with a hanging mercury drop electrode as working electrode, a platinum wire as counter electrode, and an aqueous $\mathrm{Ag} / \mathrm{AgCl}$ reference electrode $(3 \mathrm{M} \mathrm{KCl})$. For DPV measurements, a modulation amplitude of $25 \mathrm{mV}$ was used. Electrochemical measurements on surfaces were performed in a custom-built glass electrochemical cell with a platinum disk as counter electrode, and the functionalized gold or silicon substrates as working electrode $\left(0.44 \mathrm{~cm}^{2}\right)$. As a reference electrode, a red rod electrode was used in aqueous solutions $(\mathrm{Ag} / \mathrm{AgCl}$, saturated $\mathrm{KCl}$ solution, Radiometer Analytical) or $1 \mathrm{mM}$ ferrocenemethanol as internal standard in acetonitrile solutions. A Faradaic cage was used to shield influences from the environment. For the stability tests with guest 4 , the guest surface coverage was determined by recording cyclic voltammograms in aqueous solutions of $1 \mathrm{M}$ sodium 
perchlorate monohydrate $\left(\mathrm{NaClO}_{4}\right)$ at scan rates of $0.2,1.0,2.0$, and $5.0 \mathrm{~V} / \mathrm{s}$ for $\mathrm{Au}$ substrates and $0.1,0.2,0.5,1.0$, and $2.0 \mathrm{~V} / \mathrm{s}$ for Si substrates. The cycles at more negative potential ranges were performed at $1 \mathrm{~V} / \mathrm{s}$. The peak currents were determined by Gaussian fitting (using linear baseline correction) in the CHI760D software. For the CV measurements of guest 5 in solution, the first scan is shown in the graphs. For the CV measurements on surfaces with 1 or with guest 5, two or three cycles were recorded, of which the last is shown in the graphs. DPV measurements on surfaces were performed with an increasing potential of $2 \mathrm{mV}$, an amplitude of $40 \mathrm{mV}$ and a pulse width of $0.1 \mathrm{~s}$.

\subsection{References}

1. I. Ganesh, Renewable Sustainable Energy Rev., 2015, 44, 904-932.

2. K. Sun, S. Shen, Y. Liang, P.E. Burrows, S.S. Mao and D. Wang, Chem. Rev., 2014, 114, 86628719.

3. J. Nowotny, C.C. Sorrell, L.R. Sheppard and T. Bak, Int. J. Hydrogen Energy, 2005, 30, 521-544.

4. J.M. Spurgeon, M.G. Walter, J.F. Zhou, P.A. Kohl and N.S. Lewis, Energy Environ. Sci., 2011, 4, 1772-1780.

5. Y.D. Hou, B.L. Abrams, P.C.K. Vesborg, M.E. Bjorketun, K. Herbst, L. Bech, A.M. Setti, C.D. Damsgaard, T. Pedersen, O. Hansen, J. Rossmeisl, S. Dahl, J.K. Norskov and I. Chorkendorff, Nat. Mater., 2011, 10, 434-438.

6. M.E. Louis, T.G. Fenton, J. Rondeau, T. Jin and G.H. Li, Comments Inorg. Chem., 2016, 36, 3860.

7. J. Seo, R.T. Pekarek and M.J. Rose, Chem. Commun., 2015, 51, 13264-13267.

8. A. Le Goff, V. Artero, R. Metayé, F. Moggia, B. Jousselme, M. Razavet, P.D. Tran, S. Palacin and M. Fontecave, Int. J. Hydrogen Energy, 2010, 35, 10790-10796.

9. P. Rodriguez-Macia, A. Dutta, W. Lubitz, W.J. Shaw and O. Rudiger, Angew. Chem., Int. Ed., 2015, 54, 12303-12307.

10. N.M. Muresan, J. Willkomm, D. Mersch, Y. Vaynzof and E. Reisner, Angew. Chem., Int. Ed., 2012, 51, 12749-12753.

11. R. Becker, S. Amirjalayer, P. Li, S. Woutersen and J.N. Reek, Sci. Adv., 2016, 2, e1501014.

12. G.A.N. Felton, A.K. Vannucci, J. Chen, L.T. Lockett, N. Okumura, B.J. Petro, U.I. Zakai, D.H. Evans, R.S. Glass and D.L. Lichtenberger, J. Am. Chem. Soc., 2007, 129, 12521-12530.

13. F. Quentel, G. Passard and F. Gloaguen, Chem. Eur. J., 2012, 18, 13473-13479.

14. J.C. Fontecilla-Camps, A. Volbeda, C. Cavazza and Y. Nicolet, Chem. Rev., 2007, 107, 4273-4303.

15. F. Quentel, G. Passard and F. Gloaguen, Energy Environ. Sci., 2012, 5, 7757-7761.

16. A.B. Sieval, R. Linke, H. Zuilhof and E.J.R. Sudholter, Adv. Mater., 2000, 12, 1457-1460.

17. J.M. Buriak, Chem. Commun., 1999, 1051-1060.

18. R. Zanoni, M. Cossi, M.F. Iozzi, F. Cattaruzza, E.A. Dalchiele, F. Decker, A.G. Marrani and M. Valori, Superlattices Microstruct., 2008, 44, 542-549.

19. A.G. Marrani, F. Cattaruzza, F. Decker, R. Zanoni, M. Cossi and M.F. Iozzi, J. Nanosci. Nanotechnol., 2010, 10, 2901-2907.

20. V. Aiello, N. Joo, J. Buckley, G. Nonglaton, F. Duclairoir, L. Dubois, J.C. Marchon, M. Gely, N. Chevalier and B. De Salvo, Surf. Sci., 2013, 612, 57-62. 
21. C.A. Nijhuis, B.J. Ravoo, J. Huskens and D.N. Reinhoudt, Coord. Chem. Rev., 2007, 251, 17611780.

22. C.Z. Zhu and L. Fang, Curr. Org. Chem., 2014, 18, 1957-1964.

23. A. Méndez-Ardoy, T. Steentjes, T. Kudemac and J. Huskens, Langmuir, 2014, 30, 3467-3476.

24. M.W.J. Beulen, J. Bügler, B. Lammerink, F.A.J. Geurts, E.M.E.F. Biemond, K.G.C. van Leerdam, F.C.J.M. van Veggel, J.F.J. Engbersen and D.N. Reinhoudt, Langmuir, 1998, 14, 6424-6429.

25. S. Onclin, A. Mulder, J. Huskens, B.J. Ravoo and D.N. Reinhoudt, Langmuir, 2004, 20, 54605466.

26. C. Lagrost, G. Alcaraz, J.F. Bergamini, B. Fabre and I. Serbanescu, Chem. Commun., 2007, 10501052.

27. A. Mulder, T. Auletta, A. Sartori, S. Del Ciotto, A. Casnati, R. Ungaro, J. Huskens and D.N. Reinhoudt, J. Am. Chem. Soc., 2004, 126, 6627-6636.

28. A. Perl, A. Gomez-Casado, D. Thompson, H.H. Dam, P. Jonkheijm, D.N. Reinhoudt and J. Huskens, Nat. Chem., 2011, 3, 317-322.

29. A. Gomez-Casado, H.H. Dam, M.D. Yilmaz, D. Florea, P. Jonkheijm and J. Huskens, J. Am. Chem. Soc., 2011, 133, 10849-10857.

30. X. Wang, K.Q. Peng, Y. Hu, F.Q. Zhang, B. Hu, L. Li, M. Wang, X.M. Meng and S.T. Lee, Nano Lett., 2014, 14, 18-23.

31. S. Pullen, H.H. Fei, A. Orthaber, S.M. Cohen and S. Ott, J. Am. Chem. Soc., 2013, 135, 1699717003.

32. B.R. Garrett, A. Awad, M. He, K.A. Click, C.B. Durr, J.C. Gallucci, C.M. Hadad and Y. Wu, Polyhedron, 2016, 103, Part A, 21-27.

33. J. Navarro-Ruiz, A. Rimola and M. Sodupe, J. Phys. Chem. C, 2013, 117, 15130-15138.

34. B. Kumar, M. Beyler, C.P. Kubiak and S. Ott, Chem. Eur. J., 2012, 18, 1295-1298.

35. C. Tard and C.J. Pickett, Chem. Rev., 2009, 109, 2245-2274.

36. M.L. Singleton, J.H. Reibenspies and M.Y. Darensbourg, J. Am. Chem. Soc., 2010, 132, 88708871.

37. A. Méndez-Ardoy, T. Steentjes, B.A. Boukamp, P. Jonkheijm, T. Kudernac and J. Huskens, Langmuir, 2016, in press, DOI: 10.1021/acs.langmuir.6b03860.

38. M. Mahon, K.W. Wulser and M.A. Langell, Langmuir, 1991, 7, 486-492.

39. S. Moussa, G. Atkinson and M.S. El-Shall, J. Nanopart. Res., 2013, 15, 1470.

40. J. Huskens, A. Mulder, T. Auletta, C.A. Nijhuis, M.J.W. Ludden and D.N. Reinhoudt, J. Am. Chem. Soc., 2004, 126, 6784-6797.

41. J. Sanabria-Chinchilla, A. Javier, D. Crouthers, J.H. Baricuatro, M.Y. Darensbourg and M.P. Soriaga, Electrocatalysis, 2014, 5, 5-7.

42. Y. Na, M. Wang, K. Jin, R. Zhang and L. Sun, J. Organomet. Chem., 2006, 691, 5045-5051.

43. L. Bertini, C. Greco, P. Fantucci and L. De Gioia, Int. J. Quantum Chem., 2014, 114, 851-861.

44. C. Amatore, J. Pinson, J.M. Saveant and A. Thiebault, J. Electroanal. Chem., 1980, 107, 59-74.

45. M.A. Fox and R. Akaba, J. Am. Chem. Soc., 1983, 105, 3460-3463.

46. C.A. Nijhuis, F. Yu, W. Knoll, J. Huskens and D.N. Reinhoudt, Langmuir, 2005, 21, 7866-7876.

47. B. Yosypchuk and V. Mareček, J. Electroanal. Chem., 2011, 653, 7-13.

48. P.R. Ashton, R. Königer, J.F. Stoddart, D. Alker and V.D. Harding, J. Org. Chem., 1996, 61, 903908. 


\section{Summary}

Silicon is an attractive semiconductor material for wide-ranging applications, especially when taking advantage of the larger surface area of silicon micro and nanowires. Surface functionalization with self-assembled monolayers of (in)organic molecules can be employed to tune the functionality of a substrate towards a desired application. Specifically, solar-to-fuel and sensing devices highly benefit from the use of oxide-free monolayers, since any silicon oxide layer functions as an insulating layer and retards electrical contact with the substrate. For this purpose, hydrosilylation can be used to couple terminal unsaturated carbon-carbon bonds, i.e., 1-alkenes or 1-alkynes, onto $\mathrm{H}$-terminated Si which leads to direct $\mathrm{Si}-\mathrm{C}$ bond formation. The research described in this thesis aims at the formation of molecular monolayers on $\mathrm{H}$-terminated silicon micro and nanowires for solar-to-fuel and sensing devices.

In the first part of this thesis (Chapters 3-4), oxide-free monolayers have been applied onto silicon micro and nanowires for solar-to-fuel devices. Chapter 3 has reported the use of molecular monolayers for simultaneous passivation and catalyst coupling. A 1-tetradecyne monolayer functioned as an electrical passivation layer, which increased the efficiency of micropillar-based solar cells. At the same time, secondary functionalization was demonstrated with a model catalyst by copper-catalyzed click chemistry onto a 1,8-nonadiyne monolayer, which could be used in the future for (photo)catalyst coupling for efficient solar fuel production. In Chapter 4, nanowires have been fabricated by metal-assisted chemical etching (MACE) to benefit from an even larger surface area compared to microwires. After fabrication, highly doped nanowires were created by three different monolayer doping techniques, where the total doping dose inside the nanowires could be tuned by changing the porosity of the nanowires.

The second part of this thesis (Chapters 5-6) has described the selective functionalization of silicon nanowires. Chapter 5 has discussed the spatioselective functionalization of silicon nanowires without the use of a masking material, based on alternating steps of MACE and monolayer formation. This resulted in nanowires with a 1,8-nonadiyne monolayer on the upper segments only, as seen by a contrast difference in scanning electron microscopy images. Secondary functionalization with azide-functionalized nanoparticles confirmed this observation by a threefold higher particle density on the top segments compared to the lower segments without a monolayer. In Chapter 6, material-selective functionalization of silicon nanowire sensors was targeted to prevent loss of analyte and increase the sensor's sensitivity. However, hydrosilylation resulted in 
nonselective functionalization, which required the use of an extra wet etching step to remove the monolayer from the oxidized areas. This increased the selectivity towards functionalization of the silicon nanowires, which were then functionalized with probe PNA for tumor DNA detection.

In the third part of this thesis (Chapters 7-8), electrochemistry on oxide-free monolayers has been tested. Chapter 7 has described the electron transfer of redox-active guest molecules at silicon electrodes with $\beta$-cyclodextrin host molecules. Whereas the packing density and extent of oxide formation were comparable for monolayers on lowly doped p-type and highly doped $\mathrm{p}^{++}$substrates, the electron transfer was more favorable on $\mathrm{p}^{++}$substrates. This indicated that the electrochemical response on the host layer is not only determined by the composition of the monolayer but also by the doping level of the substrate. This proof of principle was further explored in Chapter 8, in which hydrogen evolution catalysts were immobilized on silicon and gold electrodes. Hydrosilylation was used to couple an alkyne-functionalized catalyst covalently onto $\mathrm{H}$-terminated silicon. Alternatively, $\beta$-cyclodextrin host monolayers were used to immobilize a guestfunctionalized catalyst on gold and silicon electrodes by supramolecular interactions. Whereas catalyst immobilization was confirmed for both routes, preliminary results only hinted at reduction events for both catalysts, and more research is needed to verify their catalytic activity after immobilization.

In summary, the results described in this thesis demonstrate the versatility of molecular monolayers on $\mathrm{H}$-terminated silicon structures. When applying a monolayer by hydrosilylation, the absence of an insulating oxide layer allows electrical contact between the functionalized headgroup of the monolayer and the substrate. This enables the fabrication of solar-to-fuel devices, in which molecular monolayers ensure electrical passivation of the surface, a controllable doping concentration, covalent or noncovalent immobilization of catalysts, and spatioselective functionalization to couple different catalysts onto the same device. For sensing devices, oxide-free monolayers establish a higher stability of the sensor owing to the direct Si-C bonds, a higher sensitivity due to the selective functionalization of the sensing area only, and a higher specificity when using supramolecular chemistry to make an analyte-specific sensor. These findings offer new perspectives for the development of stabilized silicon micro/nanosystems with engineered functionalities. 


\section{Samenvatting}

Silicium is een aantrekkelijk halfgeleidermateriaal voor uiteenlopende toepassingen, vooral wanneer gebruikgemaakt wordt van het grotere oppervlak van siliciummicro- en -nanodraden. Oppervlaktefunctionalisatie met zelf-assemblerende monolagen van (an)organische moleculen kan gebruikt worden om de functionaliteit van een substraat af te stemmen op een gewenste toepassing. Vooral zonlicht-naar-brandstof-apparaten en sensoren profiteren sterk van het gebruik van oxidevrije monolagen, aangezien een siliciumoxidelaag functioneert als een isolator en daardoor het elektrisch contact met het substraat belemmert. Hydrosilylering kan gebruikt worden om eindstandig onverzadigde koolstof-koolstofverbindingen, d.w.z. 1-alkenen of 1-alkynen, aan H-getermineerd silicium te binden, hetgeen leidt tot Si-C-bindingen. Het onderzoek beschreven in dit proefschrift heeft als doel om moleculaire monolagen te maken op $\mathrm{H}$-getermineerde siliciummicro- en -nanodraden voor zonlicht-naar-brandstof-apparaten en sensoren.

In het eerste deel van dit proefschrift (Hoofdstukken 3-4) zijn oxidevrije monolagen toegepast op siliciummicro- en -nanodraden voor zonlicht-naar-brandstof-apparaten. In Hoofdstuk 3 is het gebruik van moleculaire monolagen voor gelijktijdige passivatie en katalysatorkoppeling beschreven. Een monolaag van 1-tetradecyn is gebruikt als een elektrische passivatielaag, waardoor de efficiëntie van een micropilaar-gebaseerde zonnecel verhoogd kon worden. Tegelijkertijd is een vervolgfunctionalisatiestap aangetoond met een modelkatalysator via kopergekatalyseerde klikchemie op een 1,8-nonadiyn-monolaag, wat in de toekomst gebruikt kan worden voor het koppelen van een (foto)katalysator voor de efficiënte productie van zonnebrandstoffen. In Hoofdstuk 4 zijn nanodraden van silicium gemaakt via metaalondersteund chemisch etsen ('metal-assisted chemical etching', MACE) om zo te profiteren van een nog groter oppervlak in vergelijking met microdraden. Na de fabricage zijn hooggedoteerde nanodraden gemaakt met behulp van drie verschillende monolaag-doteringstechnieken, waarbij de totale doteringsdosis in de nanodraden gecontroleerd kon worden door de porositeit van de nanodraden te variëren.

In het tweede deel van dit proefschrift (Hoofdstukken 5-6) is de selectieve functionalisatie van siliciumnanodraden beschreven. Hoofdstuk 5 behandelt de selectieve functionalisatie van siliciumnanodraden zonder het gebruik van een maskerend materiaal, gebaseerd op afwisselende stappen van MACE en monolaagvorming. Dit resulteerde in nanodraden met een 1,8-nonadiyn-monolaag op alleen de bovenste 
segmenten, zoals blijkt uit een contrastverschil in de rasterelektronenmicroscoopplaatjes. Een vervolgfunctionalisatiestap met azide-gefunctionaliseerde nanodeeltjes bevestigde deze observatie met een drie keer hogere deeltjesdichtheid op de bovenste segmenten in vergelijking met de onderste segmenten zonder monolaag. In Hoofdstuk 6 is materiaalselectieve functionalisatie van siliciumnanodraadsensoren bekeken om het verlies van analyt te voorkomen en zo de gevoeligheid van de sensor te verhogen. Hydrosilylering resulteerde echter in niet-selectieve functionalisatie, waardoor het gebruik van een extra, natte-etsstap nodig was om de monolaag te verwijderen van de geoxideerde gebieden. Deze extra stap verhoogde de selectiviteit voor functionalisatie van de siliciumnanodraden zonder het omliggende siliciumoxide te functionaliseren. De nanodraden zijn daarna gefunctionaliseerd met probe-PNA voor de detectie van tumor-DNA.

In het derde deel van dit proefschrift (Hoofdstukken 7-8) is elektrochemie op oxidevrije monolagen getest. Hoofdstuk 7 beschrijft de elektronoverdracht van redoxactieve gastmoleculen op siliciumelektrodes met $\beta$-cyclodextrine-gastheermoleculen. De pakkingsdichtheid en de hoeveelheid oxidevorming waren vergelijkbaar voor monolagen op laaggedoteerde $\mathrm{p}$-type en hooggedoteerde $\mathrm{p}^{++}$substraten, maar de elektronoverdracht was gunstiger op $\mathrm{p}^{++}$substraten. Hiermee is aangetoond dat de elektrochemische respons op de gastheermonolaag niet alleen wordt bepaald door de samenstelling van de monolaag, maar ook door het doteringsniveau van het substraat. Dit principe is verder onderzocht in Hoofdstuk 8, waarin katalysatoren voor waterstofproductie zijn geïmmobiliseerd op silicium- en goudelektrodes. Hydrosilylering is gebruikt om een alkyngefunctionaliseerde katalysator covalent te binden op $\mathrm{H}$-getermineerd silicium. Daarnaast zijn $\beta$-cyclodextrine-gastheermonolagen gebruikt om een gastgefunctionaliseerde katalysator op goud- en siliciumelektrodes te immobiliseren door middel van supramoleculaire interacties. Hoewel immobilisatie van de katalysator is bevestigd voor beide routes, lieten de voorlopige resultaten alleen sporadisch reductieve activiteit zien voor beide katalysatoren. Meer onderzoek is nodig om de katalytische activiteit te verifiëren na immobilisatie.

Samenvattend, de in dit proefschrift beschreven resultaten tonen de veelzijdigheid aan van moleculaire monolagen op H-getermineerde siliciumstructuren. Wanneer een monolaag wordt aangebracht via hydrosilylering, dan zorgt de afwezigheid van een isolerende oxidelaag ervoor dat elektrisch contract tussen de functionele eindgroep van de monolaag en het substraat mogelijk is. Dit zorgt er op zijn beurt voor dat de fabricage van zonlicht-naar-brandstof-apparaten mogelijk wordt, waarbij moleculaire monolagen de volgende functies kunnen verzorgen: elektrische passivatie van het oppervlak, een controleerbare doteringsconcentratie, covalente of niet-covalente immobilisatie van katalysatoren en selectieve functionalisatie om verschillende katalysatoren op hetzelfde 
apparaat te binden. Voor sensoren kunnen oxidevrije monolagen zorgen voor een hogere stabiliteit van de sensor vanwege directe Si-C-bindingen, een hogere gevoeligheid door de selectieve functionalisatie van alleen het sensorgebied en een hogere specificiteit wanneer supramoleculaire chemie wordt gebruikt om een analyt-specifieke sensor te maken. Deze bevindingen bieden nieuwe perspectieven voor de ontwikkeling van gestabiliseerde siliciummicro- en -nanosystemen met ontworpen functionaliteiten. 


\section{Acknowledgments}

Although I have always said that I would never become a doctor (physician), I will hopefully receive my doctor's title soon. As I could not have done that on my own, I am grateful to finish this thesis with an acknowledgments section. This is the section that, I admit, I always read first when I receive a thesis. In addition to the acknowledgments that were already listed per chapter, there are many people that I would like to thank for their contribution to my $\mathrm{PhD}$ period.

Om meteen te beginnen met de belangrijkste: Jurriaan, ik wil jou van harte bedanken als mijn promotor. Ik weet nog hoe verbaasd ik in het begin was dat 'een professor' smileys in zijn e-mails zette. :- Daar kijk ik inmiddels niet meer van op, maar je blijft me positief verrassen met jouw kijk op de wetenschap, je managementkwaliteiten en nieuwe ideeën. Ook als ik dacht dat ik alle opties had geprobeerd, kwam jij met een nieuw idee uit onverwachte hoek. Ik waardeer het erg dat je me de vrijheid hebt gegeven om mijn eigen keuzes te maken. Bedankt voor al je advies, snelle nakijkwerk en je bewonderenswaardige eigenschap om altijd klaar te staan voor je PhD studenten!

Other inspiration sources, fruitful discussions, and new ideas came from the subgroup meetings we had, both within and outside MnF. Therefore I like to thank all members of the electronics and solar meetings, the assembly meetings, the MnF/BNT colloquium, the TBSC group, and the Nanopil team for their input. In het bijzonder wil ik Wouter graag bedanken voor alle brainstormsessies, hulp in de cleanroom, het zijn van een vraagbaak en de (tevergeefse) volhardendheid voor het aanbieden van thee in de ochtend. Ook hartelijk dank voor het doorlezen van mijn proefschrift en de nuttige opmerkingen die daaruit voortgekomen zijn. Rick, bedankt voor het wegwijs maken in de cleanroom en het fabriceren van zonnecellen op aanvraag. Ik ben blij dat de dreiging om te stoppen met het zonnecelproject heeft geholpen. Alexander, although our joint project was 'high risk, no gain', we had a lot of fun in the lab. Thank you for sharing your knowledge on electrochemistry, band levels, and p-n junctions.

Nienke, bedankt voor jouw inzet in je uitdagende masterproject onder begeleiding van Rick en mij. Jouw enthousiaste en goed doordachte manier van werken heeft de basis gelegd voor Hoofdstuk 3. Liang, thank you for being not only a nice fumehood mate but also a good collaborator for the project that resulted in Chapter 4. Rik, dankzij jou is mijn biologiekennis weer flink bijgespijkerd binnen het Nanopil project. Bedankt dat we regelmatig konden sparren over de rare resultaten met PNA/DNA hybridisatie (Hoofdstuk 6). Alejandro, thank you for synthesizing several compounds, which led to the results 
described in Chapters 7 and 8. Esther, Riccardo, and René, thank you for our joint project on hydrogen evolution catalysts (Chapter 8) and the fruitful brainstorm meeting in Amsterdam. René, bedankt voor je optimisme en altijd enthousiaste mailtjes.

Voor de karakterisatie van samples wil ik graag Jurgen (SIMS), Gerard (XPS), Mark (SEM), Henk (FIB) en Frans (FIB) bedanken. Clemens, hartelijk dank voor je hulp bij de zoektocht naar een IR accessoire en je ondersteuning bij de ATR-IR metingen en de fluorescentiemicroscoop. I would like to thank Robert and Sun-Young for their help with the J-V measurement set-up at the PCS group. Raquel, Gülistan, and Milou, thank you for helping me with confocal microscopy.

Binnen MnF wil ik graag Nicole en Izabel bedanken voor alle hulp bij organisatorische zaken, papierwerk en conferenties. Marcel, Richard, Regine en Bianca, hartelijk dank voor jullie hulp in het dagelijkse lableven. Marcel, bedankt voor je inventieve ideeën over reactieopstellingen, en Richard, bedankt voor je hulp bij mijn vraagstukken over organische chemie. Daarnaast wil ik graag de hele cleanroomstaf bedanken voor alle ondersteuning en het onderhouden van de cleanroom. Tibor, thank you for proofreading my thesis. I also thank the committee members for reviewing my thesis and being present at my defense.

I would like to thank the entire MnF/BNT group for the nice atmosphere during the last 5.5 years. To avoid an extremely long list of names (and to avoid forgetting someone), I will not mention everybody by name. Thanks to everyone, including the cleaning staff, who gave me a good start of the day with a cheerful "good morning", even when it was not yet 8 o'clock. Mirande, bedankt voor je vrolijke telefoontjes als de broodjes bezorgd waren. Thanks to all the lab mates of lab 1 for keeping up a good spirit. De Nederlandse koffiegroep wil ik bedanken als proefpersonen voor de baksels van het weekend. Daarnaast wil ik graag mijn paranimfen, Milou en Rianne, bedanken voor alle hulp bij de laatste loodjes van mijn promotie en het afronden van mijn proefschrift. Maar eigenlijk is dat alleen de kers op de taart en wil ik jullie nog meer bedanken voor alle tijd daaromheen, inclusief alle lachmomenten tijdens de pauzes, in het lab en daarbuiten!

Last but not least, wil ik graag mijn ouders, Gerard, Astrid, familie en vrienden bedanken voor hun steun, vertrouwen en interesse in mijn onderzoek. Ik hoop dat dit boekje een klein beetje antwoord geeft op de vraag "wat doe je nou eigenlijk?".

- If 'Plan A' didn't work, the alphabet has 25 more letters (Anonymous)

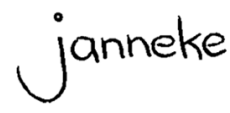

Enschede, April 2017 


\section{About the author}

Janneke Veerbeek was born in Noordoostpolder, the Netherlands, on February $28^{\text {th }}, 1990$. She studied Chemical Engineering at the University of Twente in Enschede, the Netherlands, where she received her BSc degree in July 2010. Her bachelor thesis was entitled 'Titania nanowires: towards hydrogen production' and was performed in the Inorganic Materials Science research group under the supervision of prof. dr. ir. André ten Elshof.

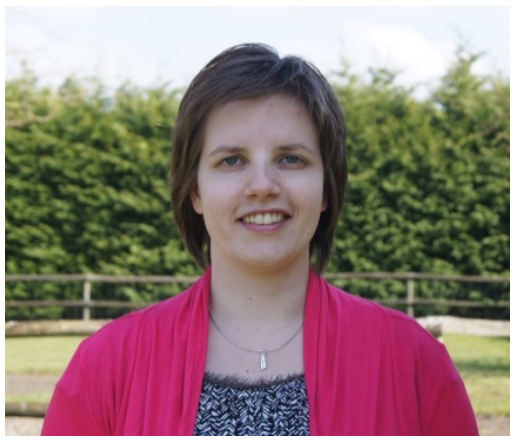

In July 2012, she received her MSc degree in Chemical Engineering, with Molecules \& Materials as a specialization track. Her internship, entitled 'One-step synthesis of loaded non-porous silica particles', was performed at the Ytkemiska Institutet, Stockholm, Sweden, under the supervision of dr. Emiel Speets. Her master thesis, entitled 'Electrochemically controlled gradients: towards directed motion of molecules', was carried out in the Molecular NanoFabrication group of prof. dr. ir. Jurriaan Huskens.

Since September 2012, she has been working as a PhD candidate at the Molecular NanoFabrication group under the supervision of prof. dr. ir. Jurriaan Huskens. The results of this research are described in this thesis.

\section{List of publications}

1. Maskless spatioselective functionalization of silicon nanowires, in preparation.

2. Selective silicon nanowire functionalization: towards early cancer DNA detection, in preparation.

3. J. Veerbeek, A. Méndez-Ardoy and J. Huskens, Electrochemistry of redox-active guest molecules at $\beta$-cyclodextrin-functionalized silicon electrodes, ChemElectroChem, 2017, in press, DOI: 10.1002/celc.201600872.

4. J. Veerbeek and J. Huskens, Applications of monolayer-functionalized H-terminated silicon surfaces: a review, Small Methods, 2017, 1, 1700072. 
5. J. Veerbeek,* L. Ye,* W. Vijselaar, T. Kudernac, W.G. van der Wiel and J. Huskens, Highly doped silicon nanowires by monolayer doping, Nanoscale, 2017, 9, 28362844. (*equal authorship).

6. J. Veerbeek, N.J. Firet, W. Vijselaar, R. Elbersen, J.G.E. Gardeniers and J. Huskens, Molecular monolayers for electrical passivation and functionalization of silicon-based solar energy devices, ACS Appl. Mater. Interfaces, 2017, 9, 413-421.

7. S.O. Krabbenborg, J. Veerbeek and J. Huskens, Spatially controlled out-of-equilibrium host-guest system under electrochemical control, Chem. Eur. J., 2015, 21, 96389644.

8. A.W. Maijenburg, J. Veerbeek, R. de Putter, S.A. Veldhuis, M.G.C. Zoontjes, G. Mul, J.M. Montero-Moreno, K. Nielsch, H. Schafer, M. Steinhart and J.E. ten Elshof, Electrochemical synthesis of coaxial $\mathrm{TiO}_{2}-\mathrm{Ag}$ nanowires and their application in photocatalytic water splitting, J. Mater. Chem. A, 2014, 2, 2648-2656.

9. J. Veerbeek, D.N. Reinhoudt and J. Huskens, Layer-by-layer assembly using host-guest interactions, in Multilayer thin films: sequential assembly of nanocomposite materials, second edition, G. Decher, J.B. Schlenoff, Eds., Wiley-VCH Verlag GmbH \& Co. KGaA (ISBN 978-3-527-31648-9), 2012, Chapter 5, 83-97.

10. J. Veerbeek, Op zoek naar de olifant: over de grenzen van modellen, in Voorbij het eind $\bullet$ Over de grenzen van de wetenschap, H. Procee, Ed., University of Twente Honours Programma Press (ISBN 978-90-365-2986-0), 2010, 214-230. 